\title{
The occurrence of Tenebrionidae (Coleoptera) in Poland based on the largest national museum collections
}

\author{
Dariusz Iwan ${ }^{*}$, Daniel Kubisz ${ }^{* *}$, Milosz A. MazuR ${ }^{* * *}$ \\ ${ }^{*}$ Museum and Institute of Zoology PAS, ul. Wilcza 64, 00-679 Warsaw, Poland, \\ e-mail: darek@miz.waw.pl \\ ** Institute of Systematics and Evolution of Animals PAS, ul. Sw. Sebastiana 9, 31-049 Cracow, Poland, \\ e-mail: kubisz@iamuzeum.pan.krakow.pl \\ ***. Center for Biodiversity Studies, Department of Biosystematics, Opole University, ul. Oleska 48, \\ 45-022 Opole, Poland, e-mail: milosz@uni.opole.pl
}

\begin{abstract}
The paper presents the results of a study on the tenebrionid beetles (more than 10,500 specimens belonging to 78 species) collected in Poland and preserved in the following national zoological collections: Institute of Systematics and Evolution of Animals PAS, Cracow (ISEA), Museum and Institute of Zoology PAS, Warsaw (MIZ) and Upper Silesian Museum, Bytom (USMB). The occurrence of 14 rarely recorded species is confirmed. The presence of 14 studied species on the ,Polish Red List of Endangered Animals" and "Polish Red Data Book of Animals" is recorded. The common species, Isomira murina (L.), as a senior synonym of I. semiflava (Kust.), should be excluded from the „Polish Red List of Endangered Animals". Two species should be excluded from Polish fauna because of an erroneous determination: Pedinus helopioides AHr. as Crypticus quisquilius (L.), and Isomira icteropa (KUst.) as Isomira murina (L.). New distributional data on the occurrence on Catalogus faunae Poloniae regions is given for 44 species. Centorus elongatus (Herbst), Cryphaeus cormutus (Fish.) and Nalassus convexus (Com.) are recorded from Poland, but not included in Polish fauna.
\end{abstract}

Key words: Coleoptera, Tenebrionidae, new records, faunistic data, Poland, museum collections.

\section{INTRODUCTION}

Nearly 25 years ago, in Volume 14 of the „Catalogus Faunae Poloniae” (KFP) (BuRAk Owski et al. 1987), a study on 20 families belonging to the superfamily of Cucujoidea, as defined at that time, was published. The work included, among others, taxa discussed in this paper. According to the previously developed outline, it contained data on taxonomy, distribution, bibliography, and on biology of species which were accounted in literature to the fauna of Poland in its present geographical borders.

The authors of KFP based on the taxonomic system, which was later modified (BouCHARD et al. 2005). For this reason, the Tenebrionidae family, as interpreted presently, comprises of three taxa treated in the catalogs of 1987 as separate families: Tenebrionidae ( 58 taxonomic names with catalog numbers) Lagriidae (2) and Alleculidae (22). The number of names is not equal to the number of species as some of them were later 
recognized as synonyms (in KFP, two names were given as two different species), e.g. Nalassus dermestoides $($ Illiger, 1798) = Helops picipes Küster, 1850; Stenomax aeneus $($ SCOPOLI, 1763) = Stenomax lanipes (Linnaeus, 1771); Isomira murina murina $($ LinNaeus, 1758) $=$ Isomira semiflava $($ KÜster, 1852). Additional information on two species occurring in Poland was published in a supplement to KFP in 2000 (Burakowski et al. 2000). It included faunistic data, not published previously, with a note „B. BuraKOWSKI*", concerning Tenebrionidae and many other families. Most of the information comes from labels on specimens studied by B. BuRAKowsK and kept mainly in the collections of the Museum and Institute of Zoology of Polish Academy of Sciences (MIZ), but also in that of the Institute of Systematics and Evolution of Animals of Polish Academy of Sciences (ISEA) and in other collections, including private ones.

In ,Fauna of Poland" checklist of species, IwaN (2004) states that 86 species of darkling beetles (according to the present interpretation of the family) can be expected to occur in Poland. The largest and most recent (especially in terms of systematics) study of Palearctic beetles (LÖBL \& SMETANa 2008) also confirms the occurrence of 86 species. However, one should be in mind that virtually all faunistic data concerning Polish beetles come from Volume 14 of the KFP.

The present work was aimed at review of materials of the largest collections in Poland, deposited in three museums: Museum and Institute of Zoology of PAS in Warsaw, Museum of the Institute of Systematics and Evolution of Animals of PAS in Cracow, and the Upper Silesian Museum (Muzeum Górnoślqskie) in Bytom. The result of the work is verification of species determination, interpretation of basic data from labels (date and place of collection), and compilation of materials for better access in the future. The collected information was placed in the database of the Polish Biodiversity Information Network (PolBIN, Polish acronym KSIB) and will be included in the „Coleoptera Poloniae" project (http://coleoptera.ksib.pl). The authors did not intend to publish a catalog of specimens in the above-mentioned collections, so no additional data were taken into account (e.g., identification number, data on biology or collection). Also the specimens labeled with illegible or incomplete data were excluded from the study. Additional analyses of various aspects of the data of the presented material, showing their possible uses, are presented in a concurrent article (TYKARSKI 2010).

Verification of species determination and faunistic data on labels of voucher specimens for previous publications, in particular for KFP, and of unpublished materials should widen our knowledge and correct inaccuracies regarding occurrence of particular species of Tenebrionidae family in Poland.

\section{STUdied COLLECTIONS}

\section{The collection of the Museum and Institute of Zoology PAS, Warsaw (Figs 1, 4)}

The material comprises tenebrionid specimens from the following collectors: Antoni BARTOSZYŃSKI (ca. 5,000 domestic species, about 20,000 voucher specimens connected 
with faunistic check lists published by that researcher), Henryk CiszKIEwICZ (collection of domestic species, about 6,000 specimens, mainly of the following families: Lucanidae, Buprestidae, Tenebrionidae, Cerambycidae and Chrysomelidae), Witold EIcHLER (collection gathered in 1889-1960, containing about 15,000 Palaearctic species; many specimens were identified by such specialists as E. Reitter, A. Fleischer, A. Schuster, G. Suworow, F. Zajtsev, V. Lutshnik, M. Pic, A. d’Orchymont, G. Olsufiev, J. MakólSKI, S. SMreCZYŃSKI), Franciszek FeJfER (beetles collected mainly in Lublin Voivodship, about 12,000 specimens of almost 4,000 species), Stefan JACZEWSKI (collection of domestic species, coming from years 1922-1940, about 4,000 specimens of some 1500 species), Friedrich KESSEL (born 1888; collection of about 150,000 specimens from various countries), Michal KLAPACZ (species not identified in most cases, about 23,000 specimens coming mainly from the territory of today's Ukraine), Mr. \& Mrs Edward and Gabriela MAZUR (collection of domestic species, containing all beetle families except of Chrysomelidae, which are deposited in ISEA, together with notes containing data on the collected specimens; about 50,000 specimens collected in 1913-1952), Otto SCHREIBER (died 1909; beetles collected mainly in the neighborhood of Nowa Sól, about 10,000 specimens), Szymon Tenenbaum (died during the II World War in the Warsaw Ghetto; the Palaearctic collection of some 150,000 specimens of at least 20,000 species), and finally the collection of former Natural History Museum in Szczecin, the core of which was the collection of the former Entomological Society in Szczecin and several separate collections (P. Franck, H. Gebien, A. LǓllwitz). Data on the collections come from the typescript by Maciej MroczKowski of May 1982, "Coleoptera beetles in the collection of the Institute of Zoology of the Polish Academy of Sciences in Warsaw" (.,Chrzqszcze Coleoptera w zbiorach Instytutu Zoologii Polskiej Akademii Nauk w Warszawie ").

\section{The collection of the Institute of Systematics and Evolution of Animals PAS, Cracow (Fig. 3)}

The historical collection is formed, in its main part, of three large collections, each of them holding several dozen thousands of specimens: the collection of Michal RYBiŃSK I (1844-1905), Boleslaw Kotula (1849-1898) and Stefan Stobecki (1859-1944). They contain beetles collected mainly in the territory of former Galicia (currently in Poland and Ukraine) and in Cieszyn Silesia. The majority of them were never precisely studied, and only a small part of the data was published (inter alia. Kotula 1872, 1873, 1874; Rybiński 1897, 1902, 1903a,b; Sтовескі 1883; Kubisz et al. 1998). The fourth large collection kept by ISEA is a collection of voucher specimens for a set of publications of Tadeusz TRella on beetles from the vicinity of Przemyśl. As it bears only a vague label of „Przemyśl vic.”, it was not taken into account in this publication, especially as some species could also be collected in today's Ukraine, which was mentioned in those publications. Some minor collections also date back to the end of the 19th and beginning of the $20^{\text {th }}$ century, e.g. the collection of Eustachy WróBLEwSKI (Lithuania, Białowieża) and Stanislaw PopeK (mainly Cracow and Babia Góra Mt.). Another interesting collection is the one of Waclaw Szymczakowski, containing xerothermic beetles, coming from the 
middle of the $20^{\text {th }}$ century, only a part of which was published (SZYMCZAKOwSKI 1960, $1965,1973)$. The remaining part of the ISEA collection consists of numerous specimens collected since the middle of $19^{\text {th }}$ century until now by many researchers mentioned in this work. Some data of the ISEA collection were used in KFP. They were listed during a preliminary survey carried out by the authors of the Catalog before its publication and marked in print as „B. BuRAKOWSKI*”, which often makes it impossible to identify the real source of the published information.

\section{The collection of the Upper Silesian Museum in Bytom (Fig. 2)}

The most interesting data come from the collection of Wojciech MaczYŃski (died 1911), created between 1884-1910. After his death, in 1929, his wife handed the collection over to the Silesian Museum of Nature History in Katowice (it was transferred to the Upper Silesian Museum after the World War II). Stanislaw SMreczýnski published a study of Curculionidae as early as in 1932 (SMRECZYŃSKI 1932), which was followed by a study of Stefan SToBiecki on Cerambycidae 8 years later (STOBIEсKI 1939). A work of Józef MAKóLSKI (1952) regarding Carabidae was published only after the war, although it was completed in 1939. Unfortunately, the collection of carabids was destroyed at the very beginning of the war, together with the whole collection of that author. The publication of MAKÓLSKI contains many comments and interpretations with respect to the possibility of collecting by W. MĄCZYŃski many southern or mountain (,,... and even alpine...") species, or ones connected with old-growth forests, in the vicinity of Warsaw. The author, analyzing occurrence of many rare and interesting species of the Carabidae family, decidedly gives credence to data coming from W. MączYŃski's collection. Beetles from the collection of MĄczYŃski were studied and published also by SzUJECKI (Steninae in 1958), SzYMCZAKowski (Catopidae in 1959) and BorowIEC (Chrysomelidae in 1992). Apart from this set of specimens, the collection of USMB is largely made up of beetles gathered by Silesian collectors before the World War II: F. KIRSCH and H. NowOTNY, and by anonymous collectors in the same period of time, herein marked as "coll. USMB”.

\section{Conventions and AbBreviations Applied}

The names of the faunistic regions are given according to "Catalogus Faunae Poloniae", and referred to as KFP regions.

Names of collectors:

$\mathrm{AB}-\mathrm{A}$. BARTOSZYŃSKI

$\mathrm{AD}-\mathrm{S}$. ADAMCZEWSKI

AG - A. Górski

AH - A. Hetschio

AJ - A. JASIŃSKI

AK - A. KUŚKA
$\mathrm{AL}$ - A. LÖLLWITZ

AM - A. MeI.KE

AN - A. WARCHALOWSKI

AO - A. Mońko

AP - A. Palaczyk

AR - A. RIEDEL

AS - A. SZEPTYCKI 


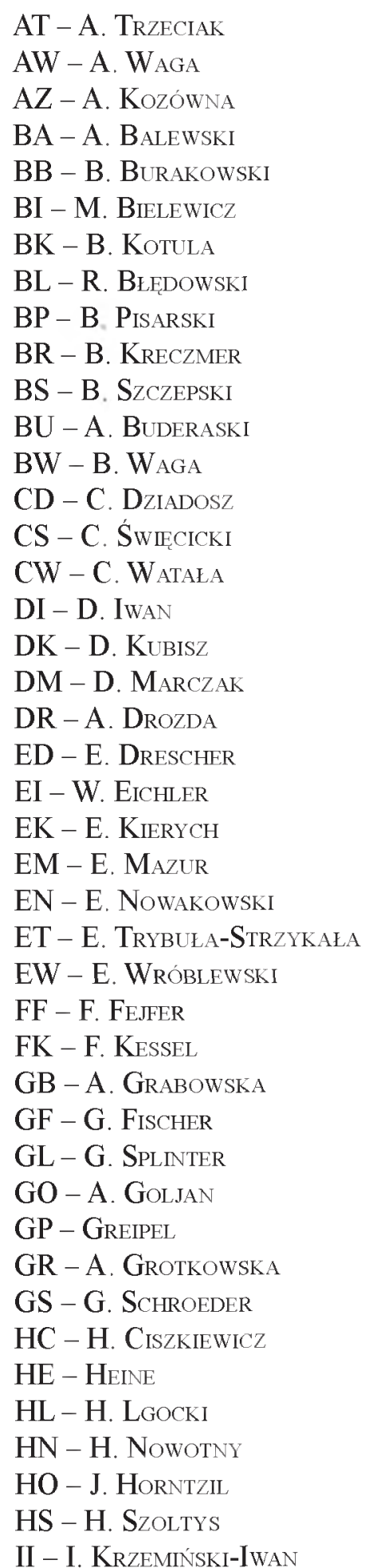

IS - I. ŚWIRSKI

JA - J. ADAMSKI

JB - J. BetLeja

JC - J. Czarnecki

JD - J. Domaniewski

JF - J. MAKÓLSKI

JG - J. M. Gutowski

JH - J. HilszczańsKI

JK - J. KuRZAWA

$\mathrm{JL}-\mathrm{J}$. ŁugOWOJ

JM - J. K. Mlynarski

JO - J. MackiEWICZ

JP - J. PAWŁOWSKI

JR - J. RAFALSKI

JS - J. ŚWIECIMSKI

JW - J. WIŚNIEWSKI

JZ - J. KOZLOWSKI

$\mathrm{KA}-\mathrm{S}$. KaDYI

$\mathrm{KB}-\mathrm{K}$. BieLEWICZ

KC - K. CZAChóR

$\mathrm{KG}-\mathrm{K}$. GALEWSKI

$\mathrm{KI}-\mathrm{F}$. KIRSCH

KJ - K. JELSKI

KK - K. KaRWOWSKI

$\mathrm{KL}-\mathrm{M}$. KŁaPACZ

KM - T. KAMIŃSKI

$\mathrm{KN}$ - J. KNIEPHOF

KO - A. Кокот

KP - KLOPFER

$\mathrm{KR}$ - J. KARPIŃSKI

KS - K. Sućko

KT - K. Kuntze

$\mathrm{KU}-\mathrm{W}$. KULERSKI

KW - J. KowaLEWSKI

KZ - J. KaCZMAREK

LB - L. BuchHolz

LD - S. LÚDERWALDT

LI - A. LIANA

LS - J. Lis

ŁP - Ł. Przybylowicz

MA - M. Bunalski

MB - M. BARTOSIK

$\mathrm{MC}$ - M. CеJKO 
MD - M. DutKa

ME - A. MACIEJEWSKI

MG - M. Galuszza

MI - M. Kosibowicz

MJ - M. JELIŃSKI

MK - M. KAZIMIERCZAK

ML - M. MŁYNARSKI

MM - M. MAZUR

MN - M. NUNBERG

MO - M. KOSIOR

MP - M. PARTYKA

MR - M. RYBIŃSKI

MS - M. STACHOWIAK

MW - M. BRAWER

MZ - M. MROCZKOWSKI

NI - W. NiEDBAEA

PA - J. PAJAK

PD - J. PoDOsKI

PK - J. PrószyŃski

PL - G. POLENTZ

PN - P. NoACK

PO - A. PokoJowCZYK

PR - J. PARUSEL

PS - P. Stachowiak

PT - S. PASTERNAK

PZ - P. Szwalko

RA - J. Razowski

RB - R. BIELAWSKI

RD - R. Dobosz

RK- R. KRóLIK

RM - R. Matusiak

RW - R. WOJAN

RY - W. RYCHTER

RZ - R. ZAMORSKI

$\mathrm{SA}-\mathrm{S}$. KaPUŚCIŃSKI

$\mathrm{SC}-\mathrm{S}$ CHEIDT

$\mathrm{SD}-\mathrm{S}$. DAJEK

$\mathrm{SE}-\mathrm{W}$. Serafiński

$\mathrm{SI}-\mathrm{A}$. SKaLSKI

SJ - S. JACZEWSKI

$\mathrm{SK}-\mathrm{S}$. KNUTELSKI

$\mathrm{SL}-\mathrm{Z}$. Ś LIWIŃSKI

$\mathrm{SM}-\mathrm{S}$. SMrecZyński jun.
SN - W. Solman

SO - W. Soltys

$\mathrm{SP}$ - S. POPEK

$\mathrm{SR}-\mathrm{W}$. StaręGa

$\mathrm{SS}-\mathrm{S}$. STOBIECKI

$\mathrm{ST}-\mathrm{S}$. Szafraniec

SU - M. Sulma

SY - Z. SZYMański

$\mathrm{SZ}$ - A. Szujecki

TE - S. Tenenbaum

TM - T. MAJEWSki II

TN - T. Spaltenstein

TO $-\mathrm{S}$. TOLL

TP - T. Plewka

TS - T. SkALSKI

TW - T. WOJAS

VW - Th. von WANKA

WA - M. WANAT

WB - W. BAZYLUK

WD - W. DOLIŃSKI

WE - M. WĘGRZECKI

WH - WEHNE

WI - B. WIŚNIOWSKI

WJ - W. JĘDRYCZKOWSKI

WK - W. KRZEMTŃSKI

WM - W. MĄCZYŃSKI

WN - W. NiESIOEOWSKI

WR - WIERZBICKA

WS - W. SZYMCZAKOWSKI

WT - W. TROJANOWSkI

WU - W. Suriedel

WW - W. WEINER

WY - WYCISŁO

$\mathrm{WZ}-\mathrm{W} . \dot{Z}_{\mathrm{Y} \mathrm{A}}$

ZA - Z ALEWSKI

ZC - Z. CAPECKI

ZI - K. ZIELIŃSKA

ZM - ZIMMER

ZP - Z. PNIEWski

ZS - Z. StebnickA

$Z T-Z$. STUGLIK

ZW - Z. WIERZBICKI

ZZ - Z. SZWARC 
Names of institutions:

ISEA - Institute of Systematics and Evolution of Animals of PAS in Cracow,

MIZ - Museum and Institute of Zoology of PAS in Warsaw,

USMB - Upper Silesian Musem in Bytom.

Other abbreviations:

KFP - Catalogus Faunae Poloniae (Burakowski et al. 1987),

nat. res. - nature reserve,

N.P. - national park.

The administrative division of public forests in Poland is as follows: forest divisions (f. div.; "nadleśnictwo") are divided into forest districts (f. distr.; "leśnictwo"), and the latter are subdivided into forest compartments (f. comp.; "oddzial").

German names of towns and villages located on the original labels have been recorded in parentheses, for example: Koszalin (Coeslin), Szczecin (Stettin).

\section{REVIEW OF SPECIES}

In the following review, the subfamilies and tribes were given in systematic order, and genera and species in alphabetical one. The taxonomic system was accepted following Bouchard et al. (2005), and the nomenclature following Catalogue of Palaearctic Coleoptera (Löbl \& SMetana 2008).

\section{LAGRIINAE LATREILLE, 1825}

\section{Laenini SeIDLITz, 1896}

\section{Laena reitteri WEISE, 1877}

Western Beskidy Mts.: Stadniki [DA42] ad Gdów, 30 IV 1988. 1 ex.. leg. BW [ISEA]; Olszowa [DA81] ad Zakliczyn. VII 1989, 1 ex.. leg. JM [ISEA]: Rytro [DV78], 19 IX 1900, 1 ex.. leg. HL [MIZ], 21 VII 1902, 5 exx., leg. WM [USMB], 21-23 VII 1902, 2 exx.. leg. SS [MIZ].

Eastern Beskidy Mts.: Uście Gorlickie [EV08], 25 V 1957, 1 ex.. leg. ZP [ISEA]; Dragaszów [EV19] ad Gorlice. 13 IX 1992. 2 exx.. leg. LP [ISEA]: Komańcza [EV76]. 6 VI 1987. 1 ex.. leg. SK [ISEA]; Holubla [FA11], 3 XI 1955, 2 exx., leg. BB [MIZ]; Przemyśl-Ostrów [FA21]. 11 X 1876 i 27 III 1877, 4 exx., leg. BK [ISEA]: Przemyśl-Lipowica [FA21]. IX 1878 and 7 XII 1878. 2 exx.. leg. BK [ISEA]: Przemyśl-Pralkowce [FA21]. 15 X 1878, 4 exx., leg. BK [ISEA].

In Poland, the species has been found only in Beskidy Mountains, which is confirmed by labels on specimens deposited in the reviewed collections (the oldest specimen dates back to 1876, but there are also ones found in the 1990s). Mature beetles have been captured rarely, but in all seasons (III, IV, V, VI, VII, IX, X, XI, XII). The species has been included in Polish Red List, DD category (PawŁowski et al. 2002). 


\section{Lagriini LatreIlle, 1825}

\section{Lagria atripes Mulsant \& Gullebeau, 1855}

Baltic Coast: Międzyzdroje [VV67], 20 VIII 1961, 1 ex., leg. SI [USMB].

Pomeranian Lake District: Bielinek nad Odrą [VU46], 30 V 1983, 5 exx., leg. BB [MIZ]. 22 VI 1984, 4 exx., leg. LB [ISEA].

Wielkopolska-Kujawy Lowland: Goluchów [YT05] ad Kalisz. 13 VI 1982, 5 VI 1989. 2 exx., leg. AM [ISEA].

Bialowieża Primeval Forest: f. comp. 375 [FD94], 24 VI 1997, 1 ex., leg. JG [ISEA].

Malopolska Upland: Grabowiec nat. res. [DA69]. 18 VI 1993 and 17 VI 1995, 3 exx.. leg. RD [USMB]; Bogucice [DA79] ad Pińczów, 22 VI 1962, 1 ex.. leg. WS [ISEA]; Owczary nat. res. [DA88] ad Busko-Zdrój. 25 VI 1999, 1 ex., leg. MM [ISEA].

Lubelska Upland: Dobre [EB68] ad Kazimierz Dolny, 28 V 2003, 1 ex., leg. DK [ISEA].

Eastern Beskidy Mts.: Węgierka [FA12] ad Druchniki, 16 VI 1965, 1 ex., leg. SO [USMB].

Stenothermic species, connected with xerotherms, found in southern edges of oak woods (Burakowski et al. 1987). Specimens captured by A. Melke in 1982 and 1989 confirm the latest reports on occurrence of this species in Wielkopolska-Kujawy Lowland (Bunalski et al. 2010). The ISEA collection (D. KuBISz coll.) includes beetles captured in Lubelska Upland (a new species for this KFP region), which supports the thesis that the species extended its range in Central Europe towards north and east at the turn of the $19^{\text {th }}$ and $20^{\text {th }}$ centuries. The MIZ collection includes voucher specimens for the report of B. Burakowski (*) of 1987 on occurrence of the species in Bielinek nad Odra nature reserve. On the other hand, specimens reported from Olawa in Lower Silesia were not found. The ISEA collection includes voucher specimens for study on Bialowieża Primeval Forest (KuBisz \& Tsinkevich 2001). The USMB collection contains a specimen found in the Baltic Coast, the region not listed in KFP.

\section{Lagria hirta (LinNAEus, 1758)}

Baltic Coast: Międzyzdroje [VV67], 10 V 1964 and 12-23 VII 1966. 3 exx., leg. AO [MIZ]: Wicko lake [VV67] ad Wolin. 8 VII 1966, 1 ex., leg. AO [MIZ]: Niechorze [WV09], 18-30 VII 1954, 4 exx., leg. AR [MIZ]; Unieście [WA71]. 21-25 VIII 1995. 1 ex., leg. JB [USMB]; Jarosławiec [WA94]. 23 VII 1955, 2 exx., leg. MZ [MIZ]; Łeba [XA66], 14 VII 1974, 2 exx., leg. MB [ISEA]: Wladyslawowo [CF37]. 21 VI 1993. 2 exx., leg. RD [USMB]: Wielka Wieś [CF37]. 26 VIII 1936. 2 exx., leg. AB [MIZ]; Sopot [CF43], VII 1950, 1 ex., leg. WE [MIZ]; Gdynia-Oksywie [CF44]. 13 VIII 1948. 1 ex., leg. RB [MIZ]; Jastarnia [CF46], 10 VIII 1934. 1 ex.. leg. JF [MIZ].

Pomeranian Lake District: Bielinek nad Odrą [VU46]. 2 VIII 1985. 2 exx.. leg. PS [ISEA]; Zatoń Dolna [VU47]. 29 VII 1957. 1 ex.. leg. EK [MIZ]; Tleń [CE14]. 30 VII 1922. 6 exx.. leg. TE [MIZ]; Plutowo [CE21] ad Chełmno, 24 VI 1964 and 20 VIII 1965, 4 exx., leg. WS [ISEA]: Czarcie Góry nat. res. ad Świecie [CE32]. 10 VII 1996, 1 ex.. leg. DK [ISEA]; Biala Góra [CE67] ad Sztum. 11 VIII 1970, 1 ex.. leg. LI [MIZ].

Masurian Lake District: Lańsk [DE64] ad Olsztyn, 24 VII 2008, 4 exx.. leg. WJ [ISEA]; Olsztyn [DE65]. 27-31 VII 1951. 3 exx.. leg. ML [ISEA]: Sępopol [EF01] ad Bartoszyce. 5 VIII 
1970, 2 exx., leg. WS [ISEA]: Wigry [FE39], 13 VII 1935. 3 exx., leg. BB [MIZ]; Puszcza Augustowska Forest: ad Plaska [FE47], 25 VI 1986, 1 ex., leg. JG [ISEA].

Wielkopolska-Kujawy Lowland: Osowo Stare [XU03] ad Szamotuly, 12 VII 1992. 1 ex.. leg. MA [ISEA]; Kolo [CC38], 1957, 1 ex., leg. BB [MIZ]; Łęczyca [CC76]. 30 VI 1937. 1 ex., leg. AB [MIZ]; Borek [CD18] ad Toruń, 25 VI 1964, 4 exx.. leg. WS [ISEA]; Ciechocinek [CD55]. 18 VII 1912, 4 exx., leg. TE [MIZ].

Mazowiecka Lowland: Podkowa Leśna [DC87], 31 VII 1937, 5 exx., leg. TE [MIZ]; Kampinoski N. P., Lipków [DC89]. 1 VIII 2005, 1 ex.. leg. WJ [ISEA]; Warszawa-Bielany [DC99], 4-9 VII 1888, 3 V 1899. 8 exx.. leg. WM [USMB]. 28 VIII and 9 IX 1948, 2 exx., leg. RB [MIZ]: Warszawa-Buchnik [DC99]. 19 VII 1956, 1 ex., leg. ZI [MIZ]; Kampinoski N. P.. Józefów [DD80], 8 VIII 2005, 1 ex., leg. WJ [ISEA]: Palmiry [DD80], 20 III 1960, 1 ex., leg. BB [MIZ]: Pomiechówek [DD81]. 8 VII 1928. 2 exx., leg. AB. 21 VIII 1949. 1 ex.. leg. WE [MIZ]; ad Przasnysz [DD97], 2 IX 1962, 1 ex., leg. BU [ISEA]: Warszawa-Powsin [EC07], 22 VII 1959. 1 ex.. leg. BB [MIZ]; Konstancin [EC07], 1929.VI.30, 1 ex., coll. MIZ; Obory [EC16], 10 VIII 1949. 1 ex., leg. AR [MIZ]: Świder [EC17]. 15 VII 1900 and 10 VII 1901. 2 exx.. leg. WM [USMB]. 12 VII 1907, 1 ex., leg. TE [MIZ]; Warszawa-Anin [EC18], 5 VIII 1949. 1 ex., leg. KG [MIZ]; Żwir (Ratajewo) [EC18], 23 VII 1951, 1 ex.. leg. BR [MIZ]: Celestynów [EC26], 29 VII 1951. 1 ex.. leg. BB [MIZ]: Opatkowice [EC31] ad Kozienice. 10 VII 1962, 1 ex.. leg. BU [ISEA]: Dębe Wielkie [EC38], 26 VII 1916, 5 exx., leg. TE [MIZ]: Karasiówka [EC61], 17 VII 1908. 3 exx., leg. TE [MIZ]; Struga [ED00], 5 IX 1957. 1 ex.., leg. BB [MIZ]; Urle [ED41], 8 VII 1900. 1 ex.. leg. WM [USMB]: Kalinowo [ED65]. 3 VIII 1933. 1 ex.. leg. AB [MIZ]: Łomża [ED79]. 30 VIII 1990, 2 exx., leg. JP [ISEA]: „Warszawa”, 19 V 1910, 3 exx., leg. WM [USMB].

Podlasie Lowland: Bedlno [FC04] ad Radzyń Podlaski, 30 V 1953, 1 ex.. leg. WB [MIZ]; Międzyrzec Podlaski [FC26], date missing, 2 exx.. leg. EI [MIZ]; Sutno [FC49] ad Mielnik. 31 VII 2008, 1 ex., leg. WJ [ISEA]: Niemirów [FC49]. 31 VII 2008, 2 exx.. leg. WJ [ISEA]: Choroszczynka [FC65] ad Tuczna, VI 1970, 2 exx., leg. JS [ISEA]; Radziwillówka [FD30] ad Siemiatycze, 1 VIII 2008, 1 ex., leg. WJ [ISEA]; Mielnik [FD30]. Uszeście nat. res., 2-28 VII 1975 and 22 VI 2001, 4 exx.. leg. ZS \& DK [ISEA]: Adamowo [FD30]. 10 VII 2009. 1 ex.. leg. WJ [ISEA]: Pilatowszczyzna [FD79]. 15-31 VII 1990, 4 exx.., leg. JP [ISEA]; Goniądz [FE12]. 6 VII 1993. 1 ex.. leg. LS [USMB].

Bialowieża Primeval Forest: Hajnówka [FD74], 7-10 VII 1930, 6 exx.. leg. TE [MIZ]. 13-27 VIII 1976, 4 exx., leg. MB [ISEA]: Czerlonka [FD84]. 20 VIII 1976, 1 ex., leg. MB [ISEA]; Teremiski [FD84], 25 VIII 1976, 1 ex.. leg. MB [ISEA]; Białowieski N. P.. f. comp. 340 [FD94]. 12 VI 1965, 4 exx., leg. BB [MIZ]: Pogorzelce [FD94]. 7 VIII 1976, 1 ex.. leg. MB [ISEA]; Bialowieża [FD94], 24 VI-2 VII 1921, 10 exx., leg. TE [MIZ], 6-30 VIII 1976, 63 exx., leg. MB [ISEA]: Białowieski N. P.. f. comp. 222 [FD95], 5 VII 1962. 1 ex., leg. AD [MIZ]: Bialowieski N. P.. f. comp. 285 [FD95]. 2 VII 1968, 1 ex.. leg. BB [MIZ].

Lower Silesia: Ligota Wielka (Ellguth Ottmachau) [XR49] ad Otmuchów. VII 1906, 2 exx., leg. ED, 15 VIII 1929, 1 ex., leg. KI [USMB]; Świdnica [XS03]. 1 VIII 1960, 1 ex., leg. KO [USMB]: Legnica [WS87], 14-15 VIII 1960, 2 exx.. leg. KO [USMB].

Upper Silesia: Pogrzebień [CA05] ad Racibórz, VI 1932 and 18 VII 1933, 3 exx.. leg. WY [USMB]; Pogwizdów [CA05] ad Racibórz, VI 1923, 1 ex.. leg. WY [USMB]; Rybnik-Paruszowiec (Paruschowitz) [CA25]. 18 VII 1909. 3 exx.. leg. SC [USMB]: Brynek [CA39|. 17 XI 1927. 1 ex., leg. KI [USMB]; Orzesze [CA45]. VI 1923, 1 ex.. leg. WY [USMB]; rez. Segiet [CA48]. 19 VII 1993, 1 ex., leg. RD [USMB]: rez. Repty [CA48], 25 VII 1993, 2 exx., leg. RD [USMB]: Bytom (Beuthen) [CA58]. 8 VI 1926 and 31 VII 1933, 5 exx.. coll. USMB. 2 VIII 1984. 1 ex.. leg. MD [USMB]; Zaborze [CA74] ad Oświęcim. 15-30 VII 1903, 1 ex., leg. SS [ISEA]; Chelmek 
[CA75]. 13-14 VIII 1883, 5 exx., leg. SS [ISEA]; Libiąż [CA75], 16 VIII 1885, 1 ex.., leg. SS [ISEA]; Jaworzno-Szczakowa [CA76]. 20 VI 1911, 1 ex.. leg. SP [ISEA]; Dąbrowa Górnicza [CA77]. VI-VII 1936, 3 exx.. leg. WM [USMB]; Bolęcin [CA95]. 30 VII 1902, 2 exx., leg. SS [ISEA]: Szymiszów (Schemischow) [CB00], 15 VII 1932, 2 exx., leg. HN [USMB].

Kraków-Wieluń Upland: Alwernia [CA94], 12 VIII 2009. 1 ex., leg. DK [ISEA]; Rzędowa Skala Mt. [CA99] ad Zawiercie. 5 VII 1997. 1 ex.. leg. DK [ISEA]; Mników [DA04] ad Kraków. 1 VIII 1897. 1 ex., leg. SS, 28 VII 1974, 1 ex., leg. MB [ISEA]: Czerna [DA05] ad Krzeszowice. 24 VIII 1902, 1 ex., leg. SS [ISEA]: Dolina Będkowska Valley [DA06] ad Kraków, 1 VIII 1968. 2 exx.. leg. WS [ISEA]: Suloszowa [DA06]. 21 VI 1990. 1 ex., leg. JP [ISEA]: Kolbark [DA08] ad Klucze. 25 VII 1969, 1 ex., leg. JP [ISEA]; Kraków-Zwierzyniec [DA14], 4 VII 1913, 3 ex.. leg. SP [ISEA]; Kraków-Przegorzały [DA14], 30 VIII 1914, 2 exx.. leg. SS [ISEA]; KrakówWola Justowska [DA14]. 10 VIII 1914, 1 ex.. leg. SS [ISEA]; Kraków-Pychowice [DA14]. 13 VII-14 VIII 1919. 26 exx., leg. SS [ISEA]: Kraków-Podgórki [DA14]. 29 VII 1962 and 20 VIII 1970, 2 exx., leg. WS \& ZS [ISEA]; Kraków-Bielany [DA14], 15 VII-5 VIII 1968, 2 exx.. leg. MG [ISEA]: Wierzchowie [DA15] ad Kraków. 12 VII 1990. 1 ex.. leg. MM [ISEA]: Ojców [DA16], 18 VIII 1970 and 18 VII 1985. 2 exx., leg. WS \& AM [ISEA]; Rząska [DA24] ad Kraków, 2 VIII 1990, 1 ex., leg. MM [ISEA].

Malopolska Upland: Pabianice [CC82]. 6 VII 1911, 1 ex.. leg. EI [MIZ]; Ruda Pabianicka [CC82], 25 VIII 1955, 1 ex., leg. SL [USMB]; Rzerzuśnia [DA27] ad Miechów, 9 VIII 1967. 1 ex., leg. WS [ISEA]: Wały nat. res. [DA47] ad Raclawice, 20 VI 2002. 1 ex., leg. DK [ISEA]: Krzyżanowice [DA68]. 9 VII 1953, 1 ex.. leg. BB [MIZ]; Skorocice [DA78] ad Busko-Zdrój. 4 VII 1961, 23 VIII 1967 and 25 VII 1987, 4 exx., leg. WS \& DK [ISEA]: Grabowiec nat. res. [DA79], 12 VII 1953, 8 VI 1954, 28 VIII 1955, 17 VII 1956, 3 VIII 1962, 9 exx., leg. BB [MIZ]: Busko-Zdrój [DA79]. 28 VI 1950. 1 ex., leg. JF [MIZ]: Chotel Czerwony [DA88]. 4 VII 1961. 1 ex., leg. WS [ISEA]; Szczekociny [DB10], date missing. 1 ex.. leg. SJ [MIZ]; Spala [DC41]. 3 VII 1947, 1 ex., leg. JC [MIZ]; Gorzyczany [EB40] ad Sandomierz, 26 VII 1971, 3 exx., leg. WS [ISEA]: Ożarów [EB43] ad Annopol, 10 VIII 1990, 2 exx.., leg. JP [ISEA]; Zapusta [EB45]. 3 VII 1992. 3 exx., coll. USMB: Góry Pieprzowe [EB51] ad Sandomierz, 22 VIII 1951-2 VII 1953, 5 exx., leg. WS [ISEA]; Dwikozy [EB52] ad Sandomierz. 7 VII 1967, 1 ex.., 1eg. WS [ISEA]; Maruszów [EB53] ad Annopol. 2 VI 1985, 1 ex.. leg. JP [ISEA]; Janowiec [EB68] ad Kazimierz Dolny, 8 VII 2003, 1 ex.. leg. DK [ISEA]; Nasilów [EB68] ad Kazimierz Dolny. 8 VII 2003, 1 ex., leg. DK [ISEA].

Świętokrzyskie Mts.: Zelejowa Góra [DB53] ad Chęciny, 7 IX 1956, 1 ex., leg. BB [MIZ]. 18 VII 1964. 1 ex.. leg. WS [ISEA]: Chęciny [DB62]. 17 VII 2009. 1 ex.. leg. DK [ISEA]; Krajno [DB83]. 11 VII 1962, 1 ex., leg. MZ [MIZ]; Łysa Góra Mt. [EB03], 26 VI 1956, 1 ex., leg. $\mathrm{BB}$ [MIZ].

Lubelska Upland: Opoka [EB63] ad Annopol. 9 VII 2003, 1 ex.. leg. DK [ISEA]; Wymyslów [EB63] ad Annopol, 9 VII 2003, 1 ex., leg. DK [ISEA]; Kazimierz Dolny [EB68], 30 V 1948, 1 ex.. leg. GO [MIZ]; Puławy [EB69], 22 VII 1951, 1 ex.. leg. BB [MIZ]; Parchatka [EB69], 9 VII 1950. 1 ex.. leg. BB [MIZ]; Bochotnica [EB78] ad Kazimierz Dolny, 9 VII 2003, 1 ex.. leg. DK [ISEA]; Stawska Góra [FB67] ad Chełm. 4 IX 1963, 2 exx.. leg. WS [ISEA]: Kosyń [FB79]. 11 VI 1994. 1 ex.. leg. MA [ISEA]; Tyszowce [FB91] ad Hrubieszów, 30 VI 1985, 1 ex.. leg. MM [ISEA].

Roztocze Upland: Zwierzyniec [FB30]. 22 VII 1911. 2 exx.. leg. TE [MIZ]; Florianka [FB30]. 2 VII 1905 and 9 VIII 1911. 6 exx., leg. FF \& TE [MIZ]; Obrocz [FB50]. 11 VII 1910. 5 exx., leg. TE [MIZ]; Nart [FB50], 22 VIII 1912, 1 ex., leg. TE [MIZ].

Western Sudety Mts.: Szklarska Poręba [WS33]. 21 VIII 1974. 1 ex., leg. MB [ISEA]; Szczawno-Zdrój [WS93|. 24 VII 1953, 1 ex.. leg. AZ [MIZ]. 
Eastern Sudety Mts.: Góry Bardzkie, Klodzka Góra Mt. [XR29], 3 VII 1949, 1 ex., leg. ZW [MIZ].

Sandomierska Lowland: Tarnów [DA94]. 1892, 1 ex.. leg. MR [ISEA]; Tarnów-Chyszów [DA94], 15 VI-16 VII 1941. 2 exx., leg. SS [ISEA]; Rzeszów [EA74]. 3 IX 1882, 1 ex.. leg. SS [ISEA]: Nisko [EA89], 26 VII 1956, 1 ex., leg. WB \& EK [MIZ]: Naklo [FA42]. 9 VII 1878. 1 ex., leg. BK [ISEA].

Western Beskidy Mts.: Kończyce Male [CA22], 4.VII 1964, 1 ex.. leg. BB [MIZ]; Tu1 Mt. [CA30], 15-19 VII 1936, 6 exx.. leg. KB [USMB]; Dzięgielów [CA31], 24 VII-10 VIII 1936. 2 exx.. leg. KB [USMB]. 8 VII 1964. 2 exx.. leg. BB [MIZ]: Równica Mt. [CA40]. VIII 1884. 1 ex., leg. BK [ISEA]: Wisla [CA40], 29 VII 1996, 1 ex., leg. WZ [USMB]; Cisownica [CA41]. 17-28 VII 1936, 2 exx., leg. KB [USMB]; Szczyrk [CA50], 4-8 VII 1954, 1 ex., leg. EI [MIZ]; Hucisko [CA61]. 6 IX 1958, 1 ex.. leg. BB [MIZ]: Pewel Mala [CA70] ad Żywiec. 12 VIII 1919. 3 exx., leg. SS [ISEA]: Wisla-Glębce [CV49], 2 IX 1958, 1 ex.. leg. BB [MIZ]: Polana [CV57]. ad Cieszyn, 25 VII 1957, 1 ex., leg. BB [MIZ]; Zawoja-Markowa [CV99]. 6 VIII 1959, 3 exx., leg. JP [MIZ]: Skawica [DA00]. 15 VII 1879. 3 exx.. leg. SS [ISEA]: Leńcze [DA02]. 15 VII 1934, 2 exx., leg. SP [ISEA]; Skomielna Czarna [DA10]. 25-28 VII 1935, 7 exx.. leg. SS [ISEA]: Skawina [DA13]. 23 VII 1911, 1 ex., leg. SP [ISEA], 11 VII 1919. 3 exx.., leg. EM [MIZ]: Wola Radziszowska [DA13]. 22 VII 1974, 1 ex.. leg. MB [ISEA]; Łapanów [DA42], date missing. 11 exx., leg. EM [MIZ]; Gruszów [DA42] ad Szczyrzyc, 23 VIII 1908, 1 ex.. leg. SS [ISEA]; Tęgoborza [DA70], 28 VII 1892, 1 ex., leg. SS [ISEA]; Polichty [DA91] ad Gromnik. 26 VI-3 VII 1998. 2 exx.. leg. DK [ISEA]; Siekierczyna [DA91] ad Ciężkowice. 3 VII 1998, 1 ex.. leg. DK [ISEA]: Obidowa [DV28] ad Nowy Targ, 12 VIII 1892. 1 ex., leg. SS [ISEA]: Piwniczna [DV77], VII 1922, 1 ex., leg. Poliński [MIZ]; Rytro [DV78], 3 VII 1892-23 VII 1900, 17 exx., leg. SS [ISEA]: Rytro [DV78]. Roztoka Wielka, 14 VII 1949, 1 ex., leg. MZ, 22-23 VII 1958. 5 exx.. leg. BB [MIZ]; Łomnica [DV87] ad Piwniczna, 17-18 VII 1898. 1 ex.. leg. SS [ISEA]; Żegiestów [DV87]. 15 VIII 1892, 1 ex., leg. SS [ISEA].

Nowotarska Dale: Zakopane [DV26]. VIII 1892, 1 ex., leg. SS [ISEA].

Eastern Beskidy Mts.: Czudec [EA53]. 15-18 VII 1917. 2 exx., leg. SS [ISEA]; Rymanów [EV69]. 21-25 VII 1955, 1 ex., leg. EI [MIZ]; Leszczawa Dolna [FA00] ad Przemyśl. 18 IX 1963, 1 ex., leg. BI [USMB]: Rybotycze [FA10], 7 VII 1992, 1 ex., leg. JP [ISEA]: Ustrzyki Dolne [FV17]. 2 VIII 1953. 1 ex., leg. BB [MIZ]: Kalwaria Paclawska [FV29]. 9 VII 1993. 5 exx., leg. JP [ISEA].

Pieniny Mts.: Kras, Długi Gronik [DV57], 17 VIII 1968, 3 exx.. leg. WS [ISEA], 15 V and 5 VII 1972. 3 exx.. leg. BB [MIZ]; Facimiech [DV57]. 1-26 VII 1971. 3 exx.. leg. BB [MIZ]: Grabczycha [DV57], 1 VII 1974, 1 ex., leg. BB [MIZ]; Trzy Korony Mt. [DV57]. 26 VII 1928. 3 exx.. leg. TE, 8 VII 1939, 1 ex.. leg. AB, 14 VIII 1955. 1 ex.. leg. WB [MIZ]: Potok Pieniński Valley [DV57], 9 VIII 1927 i 6 VII 1928, 2 exx., leg. TE [MIZ]: Wąwóz Sobczański [DV57]. 2224 VII 1972, 1 ex.. leg. ZS [ISEA]; Sromowce Wyżne [DV57], 5 VIII 1956. 1 ex., leg. KU [MIZ]; Krościenko [DV57]. 29 VI 1924 and 19 VII 1925, 3 exx., leg. TE [MIZ]: Szczawnica [DV67]. $19^{\text {th }}$ century. 1 ex.. leg. AW [ISEA]: „Pieniny Mts.”. 7 VI 1982. 1 ex.. leg. JM [ISEA].

Bieszczady Mts.: Habkowce [EV95]. 25 VII 1969, 1 ex., leg. BB [MIZ]; Jabłonki [EV95]. 10-25 VII 1967 and 5-15 VII 1968, 2 exx., leg. BI [USMB]; Baligród [EV96]. VII 1956 and 16 VIII 1958. 3 exx.. leg. TO [MIZ]: Dziurkowiec Mt. [FV03]. 10 VIII 1986. 2 exx.. leg. SZ [ISEA]: Wetlina [FV04]. 10-20 VII 1968, 2 exx.. leg. BI [USMB]: Dwernik [FV15], 6 IX 1962. 2 exx.. leg. CD \& SR [MIZ]; Tarnica Mt. [FV23]. 25 VI 1953, 1 ex., leg. BB [MIZ]: Krzemień Mt. [FV23]. 29 VII 1953, 3 exx.. leg. BB [MIZ]: Terebowiec Valley [FV23]. 6 VIII 1968. 4 exx.. leg. JP [ISEA]: Polonina Caryńska [FV23], 30 VII 1953, 1 ex., leg. BB [MIZ]. 
Tatry Mts.: Chocholowska Valley [DV15], 4 IX 1949. 1 ex.. leg. RB [MIZ]; Zakopane. Droga pod Reglami [DV25], 1-18 VIII 1950. 1 ex.. leg. WE [MIZ].

A popular species in Poland, numerously represented in the collections. It can be now confirmed that the species is found in the whole territory of Poland, except of higher mountain levels. A specimen found in 1949 by Z. WIERZBICKI (MIZ) only formally supports the occurrence of the species in Eastern Sudety Mts. (until recently, the only place where no finding of $L$. hirta has been reported, was Trzebnickie Hills). The MIZ collection includes numerous voucher specimens for all reports of B. BURAKOwsKi (*) from KFP (specimens captured in Nowotarska Dale are preserved by ISEA; the name Jaroslawiec, Pomeranian Lake District, was changed in print into „Jaroslawice”).

\section{Belopini ReITter, 1917}

\section{Centorus elongatus elongatus (HERBST, 1797)}

(Fig. 5)

Mazowiecka Lowland: Świder [EC17]. 15 VII 1900, 3 exx., leg. WM [USMB].

The species C. elongatus comprises two subspecies: C. elongatus elongatus (Herbst, 1797) and C. elongatus ecalcaratus (SEIDLITz, 1896) occurring in Europe (Sicily, Malta) and northern Africa. It has not been reported from Poland before. According to the Catalogue of Palaearctic Coleoptera (Löbl \& SMETANa 2008) C. e. elongatus is a Mediterranean subspecies, found in Turkey, Italy (Sardinia), France, Spain, Portugal, Madeira Island, Canary Islands, Morocco. The closest from Poland event of its occurrence is given by SEIDLITz (1896) from Austria (Steiermark), with a remark that identification could be erroneous. The beetles are connected with salt marshes. Its larvae develop in soil. The publication of the data from W. MĄCZYŃsKi`s collection is aimed at presentation of the actual state, i.e., presence of collected specimens which were probably introduced to Poland from abroad. Similarly as in the event of Cryphaeus cornutus (FiscH.) and Nalassus convexus (COM.), the species should not be included in Polish fauna, but it is possible that the specimens deposited in USMB have been indeed captured in Poland.

\section{TENEBRIONINAE LATREILLE, 1802}

Palorini Matthews, 2003

\section{Palorus depressus (FABRICIUS, 1790)}

Mazowiecka Lowland: Warszawa-Bielany [DC99]. 24 VI 1885, 12 V 1894, 30 V 1897, 22 VII 1898, 3 V 1899, 3 V 1900, 6 exx., leg. WM [USMB], $24 \mathrm{~V}$ 1951, 23 I and 31 VIII 1953, 10 
V 1955, 4-11 II 1959, 8 II and 13 IX 1962, 28 XI 1965, 6 VI 1966, 7 XII 1969, 19 exx., leg. BB. 17 V 1957, 1 ex.. leg. MZ [MIZ]: Warszawa-Mlociny [DC99]. 29 XI 1953, 1 ex.. leg. BB [MIZ]: Warszawa-Natolin [EC08], 10 VI 1898, 1 ex., leg. WM [USMB]: Świder [EC17], 15 VII 1900. 1 ex., leg. WM [USMB]; Urle [ED41], 11 VI 1902, 1 ex., coll. MIZ.

Lower Silesia: Buczyna Szprotawska nat. res. [WT50] ad Przemków, 19 V 2007, 1 ex., leg. $\mathrm{RD}$ [USMB].

Kraków-Wieluń Upland: Kraków [DA24], the building of Agricultural Department of Agricultural University, 1 VI 1984, 1 ex., leg. DK [ISEA].

The MIZ collection includes voucher specimens for reports of B. BURAKOWSKI (*) (Warsaw: Bielany, Młociny). A specimen from ISEA collection (D. KuBISz coll.) proves the possibility of occurrence of that species in Kraków-Wieluń Upland. The beetle often moves from its primary localities (rotten tree bark, decaying wood of broadleaf trees) to human settlements, and is considered storehouse pest. B. BURAKOwsKi captured mature beetles and larvae under oak bark and in hard wood of horse-chestnut (data on labels of specimens deposited at MIZ).

\section{Palorus ratzeburgii (Wissmann, 1848)}

Baltic Coast: Gdynia [CF44]. 9 VIII 1938, 1 ex.. leg. AB [MIZ].

Wielkopolska-Kujawy Lowland: Łęczyca [CC76], 21 X 1936, 1 ex., leg. AB [MIZ].

A species very rarely recorded in Poland, mainly synanthropic, although occasionally found outdoor, living under tree bark. The MIZ collection lacks voucher specimens for the report of B. Burakowski (*) of KFP from Siennica, while specimens from Łomianki belong in fact to $P$. subdepressus WoLL. (see below). No voucher specimens were found also for the reports from Warsaw, the work of TENENBAum (1931). In light of the above, the occurrence of this species in Mazowiecka Lowland needs confirmation.

\section{Palorus subdepressus Wollaston, 1864}

Wielkopolska-Kujawy Lowland: Poznań [XU30], the garden of Department of Entomology of Agricultural University, 16 XI 1981, 1 ex., leg. DI [ISEA].

Mazowiecka Lowland: Łomianki [DC99]. 4 IV 1953, 2 exx.. leg. BB [MIZ].

Described in literature as storehouse pest. Until recently, only one specimen has been reported from Poland, captured in Poznan, in a pile of slightly decayed bran (KUBIsz \& Szwalko 1994), and then reported from Puszcza Notecka Forest (Konwerski \& Przewoźny 2001). Specimens from Lomianki were erroneously identified in KFP (Burakowski*) as $P$. ratzeburgii (Wissm.). This is the first report on this species from Mazowiecka Lowland. 
Toxicini LACORDAIRE, 1859

\title{
Cryphaeus cornutus (FISCHER von W ALDHEIM, 1823)
}

(Fig. 7)

\begin{abstract}
Kraków-Wieluń Upland: Kraków: Botanical Garden [DA24]. 13 VI 1937, 1 ex., leg. SP [ISEA].

A species known mainly from the eastern Mediterranean region, from Caucasus to France, reaching Hungary in the north. Basing on the above-mentioned specimen, STEBNICKA (1991) included this species into her identification key of Polish Tenebrionidae (not giving precise data); she decided however that the specimen had been brought to Poland from abroad. As no further occurrences of this species were reported since then, it should not be included in the fauna of Poland.
\end{abstract}

\section{Bolitophagini KIRBY, 1837}

\section{Bolitophagus interruptus ILLIGER, 1800}

(Fig. 6)

Roztocze Upland: Panasówka [FB30], 20 VIII 1911, 17 exx.. leg. EI \& TE [MIZ]; Guciów [FB40]. 23 VIII 1912. 1 ex.. leg. TE [MIZ]: Nart [FB50]. 20 VI 1911. 1 ex.. leg. FF [MIZ].

A species not observed in Poland for many years. The MIZ collections includes only voucher specimens for reports published in the work of TENENBAUM (1913); there are no specimens coming from Poland in other collections. The species has been included in Polish Red List, DD category (Pawlowski et al. 2002).

\section{Bolitophagus reticulatus (LinNAEUs, 1767)}

Baltic Coast: Stepnica [VV74], 20 VII 1973, 1 ex., leg. MI [ISEA].

Pomeranian Lake District: Bielinek nad Odrą [VU46]. 3 VIII 1984, 4 exx.. leg. DI [USMB].

Masurian Lake District: Bachotek [CE91], 30 VII 1983, 14 exx., leg. MN [MIZ]; Łańsk [DE63]. 12 XI 2004. 1 ex.. leg. WJ [ISEA]: Kulik [EE43] ad Pisz. 22 X 1962. 1 ex.. leg. BB [MIZ]; f. distr. Boczki [FF02], 29 III 1955 i 15 X 1957, 4 exx., leg. BB [MIZ].

Wielkopolska-Kujawy Lowland: Sieraków [WU73], 23 VIII 1987, 1 ex.. leg. DI [USMB]; Średnica [WU96] ad Czarnków, 2 X 1983. 8 exx.. leg. TM [ISEA]; Krotoszyn [XT84]. 11 II 1990, 1 ex.. leg. AM [ISEA].

Mazowiecka Lowland: Kampinoski N. P., Ławy [DC79]. 23 X 1960, 1 ex., leg. BB [MIZ]; Kampinoski N. P.. Lipków [DC89]. 3 VII 2003, 2 exx., leg. WJ [ISEA]; Kampinoski N. P.. Wywrotnia Góra [DC89]. 1 X 1966, 1 ex.. leg. BB [MIZ]; Dziekanów Leśny [DC99]. 5 IV 1959. 6 exx.. leg. BB [MIZ]; Kampinoski N. P., Granica nat. res. [DD80], 11 IX 2003, 1 ex., leg. WJ [ISEA].

Podlasie Lowland: Kopna Góra [FE60] ad Supraśl. 3 V 1986. 1 ex.. leg. DK [ISEA]. 
Bialowieza Primeval Forest: Topiło [FD73], 26 V 2003, 2 exx., leg. RD [USMB]; Topilo. f. comp. 665D [FD73], 25 VIII 2003, 1 ex., leg. EN [ISEA]: Stara Judzianka [FD74] ad Hajnówka. 29 VII 2001. 1 ex., leg. WJ [ISEA]; f. comp. 424A [FD84], 1 ex.. leg. JG [ISEA]; Bialowieża [FD94], 9 VII 1920, 28 III 1921, 27 VII 1921, 14 VII 1930, 17 exx.., leg. TE [MIZ], 30 V 1934. 3 exx., leg. HC [MIZ], 28 IX-5 X 1950, 12 exx., leg. BB [MIZ]. 16 VIII 1976, 75 exx., leg. MB [ISEA]: f. comp. 340 [FD94]. 3 IV 1966 i 19 IV 1967. 2 exx.. leg. BB [MIZ]: f. comp. 342 [FD94], 4 V 1964, 2 exx., leg. BB [MIZ]; Kosy Most [FD95], 28 VII 1986, 1 ex.. leg. DK [ISEA]: Białowieski P. N., 21 III 1949, 8 exx.., leg. ZW [MIZ].

Lower Silesia: rez. Buczyna Szprotawska [WT50] ad Przemków. 19 V 2007. 18 exx.. leg. RD [USMB].

Upper Silesia: Rybnik [CA25]. 12 VII 1934, 10 exx.., leg. KL [MIZ]; Brynek [CA39]. 22 III 1927. 2 exx.. leg. HN [USMB]; Katowice-Murcki (Emmanuelssegen) [CA66]. 6 VI 1911 and 15 VI 1913, 4 exx., leg. GP, 6-20 VI 1928, 125 exx., leg. KI, V 1941. 1 ex., coll. USMB.

Kraków-Wieluń Upland: Zloty Potok [CB81], 20 VII 1900, 2 exx., leg. HL [USMB]: Kraków [DA24]. 3 X 1887, 13 exx.. leg. KJ [ISEA]: Kraków: Park Jordana [DA24]. 24 III 1896. 1 ex., leg. SS [ISEA]; „Częstochowa vicinity”, 7 VI 1934, 1 ex., leg. HC [MIZ].

Malopolska Upland: Tunel [DA28] ad Miechów. 15 IV 1985, 1 ex.. leg. JM [ISEA]; Bliżyn [DB86]. 15 VI 1968. 1 ex.. leg. SA [ISEA]: Spala [DC41]. 9 VI 1994. 1 ex.. leg. JK [ISEA].

Świętokrzyskie Mts.: Pasmo Orlowińskie. Zamczysko nat. res. [DB82]. 8 VII 1983, 1 ex., leg. LI [MIZ]: Łysica Mt. [DB93]. 26 X 1955, 15 IX 1968, 28 X 1968, 7 exx., leg. BB [MIZ]: Święty Krzyż Mt. [EB03]. 20 VI 1983. 1 ex.. leg. JM [ISEA]. 20 VI 1984. 2 exx.. leg. DM [MIZ]; „Еysogóry”, 2 VIII 1956, 1 ex., leg. AR [MIZ].

Roztocze Upland: Florianka [FB30], 20 VI 1906, 3 exx., leg. FF [MIZ]; Panasówka [FB30], 20 VIII 1911. 15 exx., leg. TE [MIZ]; Zwierzyniec [FB30]. Bukowa Góra. 2 VI 1993, 5 exx., leg. DI [USMB]; Guciów [FB40], 23 VIII 1912, 1 ex., leg. TE [MIZ]; f. distr. Grele [FB40]. 14 VIII 1911, 7 exx., leg. TE [MIZ]; Obrocz [FB41], 25 VIII 1912, 1 ex.. leg. FF [MIZ]; Nart nat. res. [FB50]. 30 VI 1985, 23 III 1986, 15 V 1987. 3 exx.. leg. DK [ISEA].

Sandomierska Lowland: Horyniec [FA66]. 1-30 VI 1888, 1-31 V i 16-20 VI 1889, 5 exx.. leg. SS [ISEA].

Western Sudety Mts.: Kornuty [WS53], 10 VI 1954, 1 ex., coll. USMB.

Western Beskidy Mts.: Równica Mt. [CA40]. VIII 1884, 4 exx., leg. BK [ISEA]; Czantoria Mt. [CA40]. VII-VIII 1884, 3 exx., leg. BK [ISEA]; Wisła [CA40]. V 1935, 2 exx., leg. WM [USMB]; Skrzyczne Mt. [CA50] ad Żywiec, VII 1884, 1 ex., leg. BK [ISEA]; Barania Góra Mt. [CV59]. 3 VIII 1873, 2 exx., leg. BK [ISEA]: Czarna Wiselka Valley [CV59]. 3 VII 1955. 1 ex., leg. BB [MIZ]; Babia Góra Mt. [CV99]. 28 VI-22 VII 1879, 9 exx., leg. SS [ISEA]; Babia Góra Mt., Hala Czarnego [CV99]. 20 VII 1948. 2 exx.. leg. ZW [MIZ]: Skawica [DA00]. 28-30 VI 1982. 2 exx.. leg. JM [ISEA]; Tęgoborza [DA70] ad Nowy Sąc. 26 VI 1892. 1 ex.. leg. SS [ISEA]; Paleśnica [DA81] ad Zakliczyn, 1987, 1 ex., leg. JM [ISEA]; Polichty [DA91] ad Gromnik, 10 X 1998, 1 ex., leg. AT [ISEA]; Młodów [DV77] ad Rytro. 12 V 1892 and 15 VII 1898. 5 exx.. leg. SS [ISEA]: Rytro [DV78]. 26 V-5 IX 1892. 21 VI-10 VII 1896. 28 VI-2 VII 1897. 19 VII 1898, 15 VII-7 VIII 1899, 25 V-23 VI 1900, 1-8 VI 1901, 10 VI-10 VII 1902. 2 VI-23 VII 1903, 19-20 VI 1919. 85 exx.., leg. SS [ISEA]: Krynica-Czarny Potok [DV97], 10 VII 1985. 1 ex.. leg. DK [ISEA]; Beskid Sądecki: Kryściówskiego Valley [DV98]. 10 VI 1989. 1 ex.. leg. AM [ISEA]: Wojkowa [EV06] ad Krynica, $20 \mathrm{~V} 1983.1$ ex., leg. DK [ISEA].

Eastern Beskidy Mts.: Radoszyce [EV76] ad Nowy Łupków. 28-30 V 1882, 3 exx., leg. BK [ISEA]; Panieński Czub [FA00] ad Bircza. 22 V 1879. 2 exx.. leg. BK [ISEA]; Rybotycze [FA10], 10 VII 1993, 1 ex., leg. JP [ISEA]: Góra Biala Mt. (Splawa) [FA10]. 7 VI and 6 VII 
1884, 6 exx.., leg. BK [ISEA]; Holubla [FA11]. $25 \mathrm{~V}$ and 15-29 VI 1884, VI 1886 and $17 \mathrm{~V}$ 1879, 19 exx.. leg. BK [ISEA]: Wapowce [FA11] ad Przemyśl. 1878, 13 exx., leg. BK [ISEA]: Olszany [FA11] ad Przemyśl. 1877-1878, 24 exx., leg. BK [ISEA]; Kaczmarowa Mt. [FA21] ad Łętownia, 28 IV 1879, 6 exx., leg. BK [ISEA]; Kamienna Laworta Mt. [FV17] ad Ustrzyki Dolne. 19 VI 1880, 3 exx.. leg. BK [ISEA]: Stebnik [FV27]. 5 VI 1880. 4 exx., leg. BK [ISEA]: Jaworniki Mt. ad Bandrów Narodowy [FV27]. 21 VI 1881. 2 exx.. leg. BK [ISEA]; Oratyk Mt. [FV27] ad Ustrzyki Dolne, 29-30 V 1880. 7 exx., leg. BK [ISEA].

Bieszczady Mts.: Jabłonki [EV95], 10 VII 1969, 1 ex.. coll. USMB; Cisna [EV95], 26 IX 1953. 1 ex.. leg. BB [MIZ]: Wetlina [FV04], 6 IX 1963. 2 exx., leg. SZ [ISEA]: Ustrzyki Górne [FV14]. 27 IX 1962. 15 IX 1958, 23 V 1966, 1 XI 1967, 4 exx., leg. BB [MIZ]: Tarnica Mt. [FV23], 19-20 VII 1880, 1 ex., leg. BK, 10 VI 1964, 11 exx., leg. JP [ISEA], 24-27 VI 1953. 7 exx.. leg. RB [MIZ]: Beskid Wolosacki [FV23]. 22 VI 1880. 1 ex.. leg. BK [ISEA]: Wolosate [FV23]. 27 VI 1953, 2 exx., leg. BB [MIZ]: Terebowiec Mt. [FV23], 27 VI 1953, 2 exx.. leg. BB [MIZ]; Bereżki [FV24], 21 VII 1968, 1 ex., leg. RB, 12 V 1970, 1 ex., leg. BP [MIZ].

Pieniny Mts.: Hulina Mt. [DV57]. 10 VIII 1928. 9 exx., leg. TE [MIZ]; Droga Pienińska [DV57], 17 VIII 1939, 31 exx., leg. TE [MIZ]; Krościenko [DV57], 9 VII 1925, 2 exx., leg. TE. 27 VII 1949, 1 ex., leg. RB [MIZ].

The species is frequently captured in Poland, which is reflected by a comparatively large amount of specimens deposited in the studied collections. It probably occurs in the whole territory of Poland, except of higher mountain levels. The collections lack only specimens from Trzebnickie Hills, Lubelska Lowland, Eastern Sudety Mts., Nowotarska Dale and Tatry Mountains, but the species has been recorded or probably occurs also in these KFP regions.

\section{Eledona agricola (HERBST, 1783)}

Baltic Coast: Rzucewo [CF36], 9-13 VII 1938, 4 exx., leg. AB [MIZ]; Mierzeja Wiślana (Frische Nehrung), date missing. 1 ex., leg. KN [USMB].

Pomeranian Lake District: Bielinek nad Odra [VU46]. 23 VII 1992. 1 ex.. leg. DK [ISEA]; Krzymowska Dąbrowa nat. res. [VU57] ad Krzymów, 9 IX 1991, 1 ex., leg. MA [ISEA]; Szczecin (Stettin) [VV62]. date missing. 3 exx., coll. MIZ.

Masurian Lake District: Orneta [DE49], 11 IX 1973, 2 exx., leg. BB [MIZ]; Sztynort [EF40]. 9 VIII 1958, 4 exx.. leg. BB [MIZ]: Ateny [FE37] ad Augustów. 14 VIII 1955. 3 exx.. leg. BB [MIZ].

Wielkopolska-Kujawy Lowland: Czempiń [XT27], 26 V 1997. 1 ex.. leg. WJ [ISEA]; Rogalin [XT39], 5 V 1984, 15 exx., leg. DI [USMB]; Łęczyca [CC76], 13 XI 1936, 6 exx., leg. AB [MIZ]: Toruń [CD37]. 22 III 1936. 2 exx., leg. AB [MIZ]: Toruń: Kępa Bazarowa [CD37]. 21 IX 1956, 9 IX 1957 i 3 IX 1962, 13 exx., leg. BB [MIZ].

Mazowiecka Lowland: Puszcza Kampinoska Forest: Zamczysko [DC69]. 25 I 1953, 2 exx.. leg. BB [MIZ]: Podkowa Leśna [DC87]. 13 VII 1934. 5 exx.. leg. TE [MIZ]: Żbików [DC88]. 24 X 1958, 1 ex., leg. BB [MIZ]; Warszawa-Leszno [DC98], 27 IV 1952, 3 exx., leg. BB [MIZ]: Warszawa-Buchnik [DC99]. 26 V 1956, 1 ex.. leg. BB [MIZ]; Warszawa-Bielany [DC99]. 5 VI 1889, 2 V 1890, 14 X 1892 and 4 X 1898, 22 exx., leg. WM [USMB]. 15 VI 1923, 3 exx.. leg. HC. 8 X 1930. 39 exx.. leg. TE. 26 V 1949. 3 exx.. leg. ZW. 15 VII and 25 VIII 1950. 1 I 1951. 10 II 1952, 11 I. 6 VI and 31 VIII 1953, 33 exx., leg. BB, 17 V 1957, 6 exx.., leg. MZ [MIZ]; Kazuń 
[DD70], 25 V 1922, 5 exx., leg. HC [MIZ]: Pomiechówek [DD71], 17 V 1928, 4 exx., leg. AB [MIZ]: Warszawa-Morysinek [EC07], 23 VI 1918, 2 exx., leg. TE. 20 X 1951. 1 ex., leg. RB. 30 X 1957 and 19 IV 1970, 16 exx., leg. BB [MIZ]: Warszawa-Wawer [EC08]. 27 VI 1898. 1 ex., leg. WM [USMB]: Warszawa-Natolin [EC08], 11 V 1899, 2 exx.. leg. WM [USMB]: Dęblin [EC51], 4 VIII 1933, 2 exx., leg. HC [MIZ]; Klembów [ED20]. 27 VII 1983, 2 exx., leg. BB [MIZ]: Szumin [ED33] ad Łochów, 19 VIII 1981, 1 ex., leg. TM [ISEA]: "Warszawa”. 20 VII 1948, 1 ex., leg. JF [MIZ].

Podlasie Lowland: Mordy [FC08] ad Siedlce, 22 VI 1991. 4 exx., leg. DK [ISEA]; Międzyrzec Podlaski [FC26], date missing, 1 ex., leg. EI [MIZ]; Niemirów [FC49] ad Mielnik, 13 V 2003, 4 exx., leg. DK [ISEA].

Bialowieża Primeval Forest: ad Czerlonka [FD84]. 30 VI 1993. 10 exx.. leg. JG [ISEA]; f. comp. 80D [FD85] 26 VI 1990, 5 exx., leg. DK [ISEA]; Bialowieża [FD94], 30 IX 1950 and 25 IX 1951, 6 exx., leg. BB [MIZ]: f. comp. 161B [FD95]. 30 IV 1990, 1 ex.. leg. RK [ISEA]: „Puszcza Białowieska”, 12 VII 1949, 6 exx.. leg. RB [MIZ].

Upper Silesia: Polomia [CA39]. 29 X 1999 and 5 XI 1999. 7 exx.. leg. RD \& HS [USMB]: Bieruń Nowy [CA74]. 13 X 1999, 9 exx.. leg. RD [USMB]: Oświęcim-Bobrek [CA74]. 13 X 1999, 5 exx., leg. RD [USMB]: Zawadzkie [CB20], 29 V 1929, 8 exx., leg. HN [USMB]: Lubliniec [CB31], 13 X 1999. 3 exx., leg. RD [USMB].

Kraków-Wieluń Upland: Klobuck [CB54], 12 VII 1924. 3 exx.. leg. HC [MIZ]; Liszki [DA14] ad Kraków, date missing. 1 ex., coll. ISEA; Kraków-Pychowice [DA14]. 8 VI 1939. 1 ex., leg. EM [MIZ]; Kraków-Dąbie [DA24], 12 VII 1900, 5 exx., leg. MR [ISEA]; Kraków: Botanical Garden [DA24]. 22-23 VI 1936. 5 V - 15 VI 1937. 14 V- 28 VI 1938. 32 exx.. leg. SP [ISEA]. 4 VI 1937, 1 ex., leg. EM [MIZ].

Malopolska Upland: Spala [DC30]. 29 VI 1912, 2 exx., leg. EI [MIZ].

Lubelska Upland: Kazimierz Dolny [EB68], 15 VIII 1947, 2 exx., leg. JF [MIZ].

Roztocze Upland: Ulów [FA58], 21 VII 1912, 42 exx. leg. TE [MIZ].

Sandomierska Lowland: Tarnów [DA94]. 1892. 6 exx.. leg. MR [ISEA]; Zaleszany [EB61] ad Sandomierz, 12-13 V 1891, 12 exx., leg. SS [ISEA]; Jaroslaw [FA24], 2 V 1888, 2 exx., leg. SS [ISEA]; Bobrówka [FA34] ad Jarosław, 15 VII-15 VIII 1960, 2 exx., leg. SO [USMB]: Nowa Grobla [FA44]. 25 IV 1888, 1 ex., leg. SS [ISEA]: Horyniec [FA66], 20 V 1888, 22 exx., leg. SS [ISEA].

Western Beskidy Mts.: Bielsko-Biala [CA51], date missing, 15 exx., coll. SN [ISEA]; Cieszyn [CA21]. VII-VIII 1873, lex.. leg. BK [ISEA].

Eastern Beskidy Mts.: Przemyśl-Budy [FA21]. VI-VII 1881, 13 exx.. leg. BK [ISEA].

The species occurs in the whole territory of Poland except of higher mountain levels. MIZ collection includes voucher specimens for the work of TENEBAUM (1913) and reports of B. BuRAKowsKi (*) from KFP (the present work gives particular data on occurrence of this species in Bialowieża Primeval Forest).

\section{Eledonoprius armatus (PANZER, 1799)}

(Fig. 8)

Eastern Beskidy Mts.: Holubla [FA11]. 29 VI 1884. 5 exx.. leg. BK [ISEA].

As a relict of old-growth forests, the species is considered extreme rarity. ISEA collections contain specimens captured in the end of the $19^{\text {th }}$ century in Eastern Beskidy 
Mountains (apart from that, it is known from several localities in 4 KFP regions). The present work contains the first (and last for the time being) published report on occurrence of this species in the above-mentioned area. It is one more confirmation that logging of forests caused extinction of this species in many countries in the XX century. For this reason, E. armatus has been counted to species probably extinct in Poland (EX? category) (Pawlowski et al. 2002).

\section{Tenebrionini Latreille, 1802}

\section{Neatus picipes (HERBST, 1797)}

\section{Pomeranian Lake District: Bielinek nad Odrą [VU46]. 23 VII 1992, 1 ex., leg. DK [ISEA].}

Wielkopolska-Kujawy Lowland: Nowa Sól (Neusalz) [WT43], 6 VI 1906 and 5 V 1908. 3 exx.. coll. MIZ: Śródka [WU83| ad Chrzypsko Wielkie. 5 V 1986. 1 ex.. leg. LB [USMB]: Krajkowo [XT38], 10 IX 1983, 29 VII and 20 VIII 1984, 5 exx.. leg. DI [USMB]; Rogalin [XT39]. 7 V 1983, 1 ex.. leg. MA. 5 V 1984. 1 ex.. leg. LB. 30 VII 1984. 1 ex.. leg. DI [USMB]: Rogalinek [XT39]. 16 V 1982, 1 ex.. leg. JW [USMB]: Czeszewo [XT78]. 9 V 1993, 3 exx., leg. LB [ISEA]: Glogówek [YR08]. 30 VI 1995. 2 exx.. leg. WZ [USMB].

Mazowiecka Lowland: Warszawa-Buchnik [DC99], 10 III 1968, 1 ex., leg. BB [MIZ]; Warszawa-Mlociny [DC99], 29 XI 1953, 1 ex., leg. BB [MIZ]: Warszawa-Żerań [DC99]. 9 V 1902, 1 ex.. leg. WM [USMB]: Warszawa-Bielany [DC99]. 18 IX 1888, 11 IV 1894. 28 VI 1897. 6 exx., leg. WM [USMB], $10 \mathrm{~V}$ 1895, 1 ex., leg. TE. 1 XI 1936, 1 ex.. leg. EM, 22 VI 1934. 1 XI 1936. 1 IX 1938, 6 exx.. leg. AB. 17 VII 1947. 3 exx.. leg. JF, 3 V 1953. 1 ex.. leg. AR. 17 V 1957, 3 exx., leg. MZ, 15 X 1950, 23 XI 1952, 23 I 1953, 13 V 1953, 9-11 X 1953, 22 XI 1953. 29 I-13 II 1955, 22 VI 1958, 4 II 1959. 26 III 1961. 27 II 1966. 21 exx., leg. BB [MIZ]: Warszawa-Morysinek [EC07], 18 V 1924, 1 ex., leg. HC, 14 I 1951, 17 IV 1955. 22 I 1961. 3 exx.. leg. BB [MIZ]: Warszawa-Saska Kępa [EC08]. 28 VII 1938. 1 ex.. leg. TE [MIZ]: Obory [EC16], 19 VIII 1950, 1 ex.., leg. BB [MIZ]; Stary Otwock [EC17], 13 VI 1928, 3 exx.. leg. TE [MIZ]: Świder [EC17]. 15 VII 1900 and 8 VIII 1901. 3 exx.. leg. WM [USMB]: „Warszawa”. 4 VI 1892. 1 ex., leg. WM [USMB], 21 VII 1948, 1 ex., leg. JF [MIZ].

Lower Silesia: Wroclaw [XS46]. Redycka str.. 5 V 1991. 1 ex.. leg. RK [ISEA].

Lubelska Upland: Parchatka [EB69] ad Pulawy, 9 VII 1950, 2 exx.. leg. BB [MIZ].

A species very widely spread in Europe, Asia and Nearctic ecozone. It is not rare, but there are few publications on it, and its occurrence has not been evidenced in many KFP regions so far. It is often captured together with species of Tenebrio genus, under bark and in hollows of broadleaved trees. The MIZ collections include voucher specimens for the reports of B. BURAKOWSKI $\left({ }^{*}\right)$ from 1987 on the occurrence of the species in Warsaw and neighboring towns, and in the vicinity of Pulawy; however, the specimen from Bialowieża has not been found. 


\section{Tenebrio molitor Linnaeus, 1758}

Baltic Coast: Wolin [VV76], 16 VII 1970, 1 ex., leg. ZZ [ISEA]; Golczewo Pomorskie [VV96], 5 VII-6 VIII 1969, 2 exx., leg. ZS [ISEA]; Ustronie Morskie [WA40]. 4 IX 1956, 1 ex.. leg. H. Jaczewska [MIZ]: Jastrzębia Góra [CF27]. 19 VIII 1936, 1 ex.. leg. SM [MIZ].

Pomeranian Lake District: Szczecin (Stettin) [VV62], 15 VII 1912, VI 1937, 10 VII 1943. 3 exx., leg. PN [MIZ]; Szczecin-Warszewo (Stettin Warsow) [VV62], 30 VII 1920, 1 ex.. leg. GS [MIZ]; Szczecin-Pogodno (Stettin. Braunsfelde) [VV62], VII 1912, 1 ex.. leg. WH, 1917, 2 exx.. leg. HE [MIZ]; Koszalin (Coeslin) [WA70]. date missing. 4 exx., leg. AL [MIZ].

Masurian Lake District: Bachotek [CE91]. 23 VIII 2010, 1 ex.. leg. II [MIZ]; Olsztyn [DE65], 31 VII 1951, 1 ex., leg. ML [ISEA].

Wielkopolska-Kujawy Lowland: Biecz (Beitsch) [VT83], 13-16 VII 1943, 3 exx., coll. USMB; Pietrzyków [VT92] ad Żary, 15 VII 1974, 1 ex., leg. TN [USMB]; Sieciejów [WT02] ad Żary, 20 VIII 1985, 3 exx.. leg. PS [USMB]; Nowa Sól (Neusalz) [WT43], date missing. 1 ex., coll. MIZ; Puszczykowo [XT29] ad Poznań, 30 VII 1982 and 5 VIII 1985. 2 exx.. leg. PS [ISEA, USMB]; Krajkowo [XT38], 6 VII 1983, 1 ex., leg. DI [USMB]; Rogalin [XT39], 22 VII 1981, 1 ex., leg. DI [USMB]: Wielkopolski N. P., f. distr. Górka, f. comp.131d [XT53], 4 VIII 1981, 1 ex., leg. LB [USMB]: Poznań [XU21]. 4 IX 1931, 1 ex.. leg. HC [MIZ]. IX 1949, 1 ex.. coll. MIZ, 21 VI 1973, 1 ex.. leg. DI, 28 VI 1985, 2 exx., leg. ET [USMB]: Wierzonka [XU41] ad Swarzędz. 16-20 VI 1941, 1 ex.. leg. WE [MIZ]; Kalisz-Wiatraki [BC94]. 28 V 1982, 1 ex.. leg. AM [ISEA]: Straszków [CC38], 1 IX 1933, 1 ex.. leg. SS [ISEA]; Łęczyca [CC76]. 27 VII 1934, 6-30 VI 1937, 3 exx., leg. AB [MIZ].

Mazowiecka Lowland: Warszawa-Bemowo [DC98], 18 VI 1987. 1 ex., leg. AM [ISEA]; Warszawa-Jelonki [DC98]. 30 VI 1997. 1 ex.. leg. WJ [ISEA]; Warszawa: Muzeum [DC98]. 24 V 1948, 1 ex., leg. Dohnet [MIZ]: Warszawa-Bielany [DC99], 2 X 1921, 1 ex.. leg. HC [MIZ]; Jabłonna [DD90], 15 VI and 18 VII 1893, 3 exx., leg. WM [USMB]; Warszawa-Saska Kępa [EC08]. 19 IV 1940. 1 ex.. leg. JF [MIZ]: Warszawa-Lazienki [EC08]. 8 VI 1908, 1 ex., leg. TE [MIZ]; Świder [EC17], 15 VII 1900, 18 VII 1901, 25 VI 1906, 3 exx.. leg. WM [USMB]. 2 V 1940, 1 ex., leg. JF [MIZ]; Warszawa-Anin [EC18], 17 VII 1949, 1 ex., leg. KG. 22 V 1951. 1 ex.. leg. MZ [MIZ]: Warszawa-Wawer [EC18]. 10 IX 1888, 2 exx.. leg. WM [USMB]: Żwir (Ratajewo) [EC18], 26 VI 1950, 1 ex., leg. BR [MIZ]; Kozienice [EC31], 19 VII 1995, 1 ex.. leg. RZ [ISEA]; Dębe Wielkie [EC38], 12 VII 1917, 1 ex., leg. TE [MIZ]: Garwolin-Parysew [EC45]. 24 IX 1933. 1 ex., leg. AB [MIZ]: Siennica [EC47], 4 XI 1928, 1 ex.. leg. AB [MIZ]: Kalinowo [ED65]. 26 VII 1932 and VI 1936, 4 exx.. leg. AB [MIZ]; „Warszawa”, 29 VI 1947. 16 VI and 20 VII 1948, 3 exx., leg. JF, I 1958, 1 ex., leg. SE, 10 VII 1959, 1 ex., leg. WE, 18 IV 1985. 1 ex.. leg. BB [MIZ].

Podlasie Lowland: Marcelin [ED50], 27 IX 1893, 1 ex., leg. WM [USMB]; Choroszczynka [FC65] ad Tuczna, VI 1970, 3 exx., leg. JS [ISEA]: Pilatowszczyzna [FD79]. 15-31 VII 1990 and 5-20 VII 1996. 2 exx.. leg. JP [ISEA].

Bialowieża Primeval Forest: Bialowieża [FD94]. 1 IV 1951. 1 ex.. leg. BB [MIZ].

Lower Silesia: Browieniec Nowy [XR98] ad Glogówek, 27 VI 2002, 3 exx., leg. JB [USMB]; Żarów (Saarau) [XS04], VII 1935, 1 ex., coll. USMB; Ząbkowice Śląskie [XS20]. 28 VII 1946. 21 VII 1948, 13 VII 1949, 3 VII 1950, 13 VII 1956, 6 exx.. leg. KL [MIZ].

Upper Silesia: Zabrze-Pawlów (Paulsdorf O/S) [BA95], 8 VII 1922, 1 ex., coll. USMB; Pogrzebień [CA05] ad Racibórz, 4 VI 1932, 2 exx., leg. WY [USMB]; Racibórz (Ratibor) [CA05]. 6 VIII 1911. 1 ex.. coll. USMB; Rybnik-Paruszowiec (Paruschowitz) [CA25]. date missing, 1 ex.. leg. SC [USMB]; Kozłów [CA27], 23 VII 1991, 1 ex.. leg. KW [USMB]: 
Maciejowice (Matwitz) [CA37], 15 VII 1927, 2 exx., leg. KI [USMB]; Bielszowice (Bielschowitz) [CA47]. 28-30 VI and 16 VII 1923, 20 VI 1927, 8 VIII 1931, 12 X 1932, 10 exx., coll. USMB: Ruda Śląska (Ruda) [CA47]. 10 VIII and 16 IX 1923, 13 VII 1924, 10 VIII 1931. 6 exx.. coll. USMB: Zabrze (Hindenburg O/S) [CA47], 15 IX 1913, 5 VII 1914, 10 VI 1921, 4 exx., coll. USMB; Kończyce [CA47], 12 VII 1961, 26 VI 1964, 24 VI 1967, 27 VI 1973, 4 exx.. leg. RY [USMB]; Czechowice-Dziedzice [CA53]. VI 1925, 1 ex.. leg. WM [USMB]; Mikolów-Jamna [CA55], 27 VI 1965, 1 ex., leg. KW [USMB]; Chorzów [CA57], 3 VII 1995, 1 ex.. leg. JB [USMB]; Bytom (Beuthen) [CA58], VII 1910 and 24.VI 1911, 4 exx., leg. GP, 9 XI 1926, 10 V 1927. 31 VII 1928. 5 exx., leg. KI. 7 VII 1933, 2 exx.. coll. USMB. V 1984. 2 VII and 1-9 VIII 1987, 7 exx., leg. MD, 25 VIII 2001, 1 ex., leg. WZ [USMB]; Chelmek [CA75], 10-16 VII 1878, 1 VII 1880, 28-30 IV 1881, 15-20 VIII 1884, 8 exx., leg. SS [ISEA]; Jaworzno-Szczakowa [CA76]. 8 VII 1910. 2 exx., leg. SS. 21 VII and 15 VIII 1912. 2 exx.. leg. SP [ISEA]: Dąrowa Górnicza [CA77]. VII 1936, 2 exx. coll. USMB; Żarki [CA84] ad Chrzanów, 20 V-15 VI 1881. 1 ex., leg. SS [ISEA]; Klekotna [CB22] ad Lubliniec. 6 VIII 1961, 1 ex.. leg. BI [USMB]: Lubliniec [CB31]. 22 VI 1994. 1 ex.. leg. RD [USMB].

Kraków-Wieluń Upland: Zakrzew [CB54] ad Klobuck. VI 1924, 1 ex., leg. HC [MIZ]; Lemańsk [CB56] ad Klobuck, 20 VII 1948, 1 ex., leg. MZ [MIZ]; Czatkowice [DA05], 30 VIII 1915. 2 exx.. leg. SS [ISEA]; Krzeszowice [DA05]. 11 V 1937. 1 ex.. leg. SP [ISEA]; Raclawice [DA06] ad Przeginia, 11 VII 1968, 1 ex., leg. JP [ISEA]; Kraków-Pychowice [DA14]. 17 V 1930. 1 ex., leg. SS [ISEA]; Kraków-Zwierzyniec [DA14]. 13 V 1906, 1 ex., leg. SS, 22 VIII 1968-21 VII 1976. 4 exx., leg. MG \& MB [ISEA]: Kraków-Sikornik [DA14]. 29 VI 1935. 1 ex.. leg. SP [ISEA]: Kraków-Przegorzaly [DA14], 7 VII 1917, 1 ex.. leg. SS [ISEA]: Ojców [DA16], 24 VIII 1913, 1 ex.. leg. EI [MIZ]: Kraków: Botanical Garden [DA24]. 30 VI 1925 and 28 VI 1935. 2 exx.. leg. SS [ISEA]; Kraków-Stare Miasto [DA24], 5-17 VII 1985, 3 exx.., leg. JP [ISEA].

Malopolska Upland: Pabianice [CC82], 20 VII and 5 VIII 1911, 2 exx.. leg. EI [MIZ]: Proszowice [DA56], 30 VII-1 VIII 1894, 2 exx., leg. SS [ISEA]; Pińczów [DA69]. VIII 1922. 2 exx.. coll. MIZ; Busko-Zdrój [DA89], 28-30 VI 1950 and 11 VII 1951. 6 exx., leg. JF [MIZ]: Rogów [DC24]. 10 X 1958, 1 ex.. leg. BB [MIZ]: Zacharzów [DC91] ad Radom. VII 1937. 1 ex., leg. A. Gretkówna [MIZ]; Radom [EB19]. 24 VI 1897, 1 ex., leg. SS [ISEA]; Sandomierz [EB51], 12 VII 1950, 1 ex., leg. MZ [MIZ].

Świętokrzyskie Mts.: Kielce-Pietraszki [DB63]. 28 VIII 1913. 1 ex.. leg. EI [MIZ]; Kielce [DB73]. 20 VII 1913, 1 ex., leg. EI [MIZ].

Lubelska Upland: Kazimierz Dolny [EB68], 19 VIII 1907, 1 ex.. leg. TE [MIZ]; Puławy [EB69]. 20 VII and 1 X 1948, 6 exx., leg. GO [MIZ]; Tyszowce [FB91]. 1933, 2 exx., coll. MIZ.

Roztocze Upland: Florianka [FB30], 15 VII 1905 and 15 V 1916. 2 exx., leg. FF [MIZ]: Obrocz [FB41]. 11 VII 1910, 2 exx., leg. TE [MIZ]: Zamość [FB52], VII 1930, 3 exx., leg. FF [MIZ].

Sandomierska Lowland: Puszcza Niepolomicka Forest: Kłaj [DA53], 1904, 3 exx., leg. MR [ISEA]: Dąbrowice [DA53] ad Bochnia, 11 VIII 1894, 1 ex.. leg. SS [ISEA]: Tarnów [DA94]. 26 VI 1973. 1 ex., leg. WT [ISEA]; Smęgorzów [EA06] ad Dąbrowa Tarnowska, 15 VII-30 VIII 1897, 8 exx., leg. SS [ISEA]; Jarosław [FA24], 20-30 VI 1888, 1 ex., leg. SS [ISEA]; Horyniec [FA66]. 1-30 VI 1889. 17 exx.. leg. SS [ISEA].

Western Sudety Mts.: Krzeszów [WS72], 24 VI 1961, 2 exx., leg. KO [USMB].

Western Beskidy Mts.: Cieszyn [CA21]. VII-VIII 1884, lex.. leg. BK [ISEA]; Czantoria Mt. [CA40]. 1880, 1 ex.. leg. BK [ISEA]: Cisownica [CA41] ad Goleszów. 17 VII 1936. 1 ex.. leg. KB [MIZ]; Zawoja [CV99]. 20-28 VIII 1936, 28 VII 1937. 28 VII-10 VIII 1938, 4 VII 1939. 
8 exx., leg. SP [ISEA]; Zawoja-Barańcowa [CV99]. 28 VII 1949. 6 exx., leg. GO [MIZ]; Babia Góra Mt. [CV99]. 5 VII 1879. 10 VII 1880. 2 exx.. leg. SS [ISEA]; Brzeźnica [DA03]. 13 VII 1918, 1 ex., leg. SS [ISEA]: Lubień [DA20]. 7 VII 1973, 1 ex.. leg. MB [ISEA]: Wrzasowice [DA23], 25-30 VII 1897, 1 ex.. leg. SS [ISEA]; Slomiróg [DA43]. VI 1912, 1 ex., leg. EM [MIZ]; Tegoborza [DA70] ad Nowy Sącz, 14 VI 1892, 1 ex.. leg. SS [ISEA]; Ruda Kameralna [DA81]. 20 VIII 1965. 1 ex.. leg. TN [USMB]: Skawa [DV29]. 14-18 VIII 1880. 1 ex.. leg. SS [ISEA]; Rdzawka [DV29], 22 VII 1998, 1 ex., leg. WZ [USMB]; Rabka [DV29], 1879. 1 ex., leg. SS [ISEA]: Piwniczna [DV77]. VII 1922, 1 ex.. leg. Poliński [MIZ]: Młodów [DV77] ad Rytro. 14 VII 1899. 1 ex.. leg. SS [ISEA]: Rytro [DV78]. 15 VII-5 VIII 1892. 22 VII 1899. 26-28 VII 1901, 4 exx., leg. SS [ISEA]: Nowy Sącz [DV79]. 28 VI 1892 and 12 VIII 1892. 2 exx., leg. SS [ISEA], 20 VII 1936, 1 ex., leg. DR [USMB]; Krynica [DV97], VIII 1937. 1 ex.. leg. $\mathrm{AB}[\mathrm{MIZ}]$.

Nowotarska Dale: Zakopane-Skibówki [DV26], 1880 and 12 VII-20 VIII 1882, 3 exx.. leg. BK [ISEA]; Zakopane [DV26], VIII 1892. 3 exx., leg. SS [ISEA]. 3 VIII 1927, 16-18 VII 1928. 3 exx.. leg. TE. 2 IX 1949. 2 exx.. leg. JF \& RB [MIZ]: Bukowina Tatrzańska [DV36]. 15 VII 1928, 1 ex., leg. HC [MIZ]. 9 VII 1960, 1 ex., leg. BI [USMB]; Lopuszna [DV38], 23 IX 1904, 1 ex., leg. SS [ISEA].

Eastern Beskidy Mts.: Gorlice [EA10]. 5 VIII 1916. 1 ex.. leg. SS [ISEA]; Kowalowy [EA31] ad Jaslo, 22 VII 1917, 1 ex., leg. SS [ISEA]; Jaslo [EA31]. VII 1922, VII 1944, 2 exx. leg. KA [ISEA]; Przemyśl-Wzniesienie [FA21]. X 1882, 2 exx., leg. BK [ISEA]; Przemyśl [FA21]. VII-VIII 1881, X 1882. IX 1886. 3 exx., leg. BK. VIII 1935. 15 exx., leg. SP [ISEA]. 3 VII 1938, 1 ex., leg. HC [MIZ].

Bieszczady Mts.: Habkowce [EV95], 14 VII 1969. 1 ex.. leg. BB [MIZ]; Baligród [EV96], 31 VII 1958, 1 ex., leg. TO [MIZ].

Pieniny Mts.: Sromowce Wyżne [DV57]. 8 VII 1939, 1 ex., leg. AB [MIZ]; Haluszowa [DV57], 20 VII 1924, 3 exx., leg. TE [MIZ]; Krościenko [DV57]. 5 VII and 8 VIII 1924, 14-17 VII 1925, 6-23 VII 1928, 8 exx., leg. TE, 27 VII 1949, 1 ex.. leg. RB, 16 VIII 1953 and 11 VIII 1972, 2 exx. leg. BB [MIZ].

Tatry Mts.: Mala Łąka Valley [DV15], 1880, 2 exx., leg. BK [ISEA]; Kuźnice [DV25], VI 1923, 2 exx., leg. EM [MIZ]: Pańszczyca Valley [DV35]. 11 VI 1915, 2 exx.. leg. EM [MIZ]: „Tatry Mountains”. VII 1912, 1 ex.. leg. EM [MIZ].

A cosmopolitan species, synanthropic, regarded storehouse pest. In Poland, it may be found in natural habitats and migrate to or from human settlements (often captured with light). Only a few specimens are labeled with particulars of place of collection, so it is usually difficult to established whether a beetle comes from a natural locality (e.g. "rotting old oak wood", "under an oak tree stump bark") or human settlements. The authors of KFP decided that the species is so frequent that there is no need to specify its localities. However, we do not share this opinion.

\section{Tenebrio obscurus FABRICIUs, 1792} [MIZ].

Pomeranian Lake District: Koszalin (Coeslin) [WA70], date missing, 2 exx.. leg. AL

Wielkopolska-Kujawy Lowland: Nowa Sól (Neusalz) [WT43]. V 1906, IX 1908, 8 VI 1909. 4 exx.. coll. MIZ; Łęczyca [CC76]. 3 VII 1938, 1 ex.. leg. AB [MIZ]. 
Mazowiecka Lowland: Świder [EC17], 15 VII 1900, 1 ex., leg. WM [USMB]; Dębe Wielkie [EC38]. 6 VII 1917, 1 ex.. leg. TE [MIZ]; Urle [ED41]. 18 VII 1907. 1 ex., leg. WM [USMB]. 19 VIII 1910. 1 ex., leg. TE [MIZ].

Upper Silesia: Żarki [CA84] ad Chrzanów. 16-20 VI 1881, 1 ex., leg. SS [ISEA].

Kraków-Wieluń Upland: Kraków [DA24], 1892, l ex.. leg. TE [MIZ].

Sandomierska Lowland: Tarnów [DA94]. 1892. 4 exx.. leg. MR [ISEA].

Western Beskidy Mts.: Piwniczna [DV77], 1-6 VI 1896, 10 VI 1898, 8 exx.., leg. SS [ISEA]; Rytro [DV78], 15 VI-22 VII 1899, 2 exx., leg. SS [ISEA]. 20 VI-20 VIII 1965, 1 ex., leg. PD [MIZ]: Nowy Sącz [DV79]. 28 VI-25 VII 1892, 2 exx., leg. SS [ISEA].

Nowotarska Dale: Zakopane [DV26]. 24-26 VII 1879, 1 ex., leg. BK [ISEA]; ZakopaneSkibówki [DV26], 1880, 1 ex.., leg. BK [ISEA]; Bukowina Tatrzańska [DV36], 17 VII 1928, 22 VII 1929. 27 VI 1930. 2 VII 1931, 5 exx.. leg. HC [MIZ].

Pieniny Mts.: Krościenko [DV57], 5 VII 1924 and 25 VII 1925, 2 exx., leg. TE [MIZ].

The species probably occurs in whole territory of Poland, but it is known from few scattered localities. It is recorded as storehouse pest, but it is also found in natural habitats (in hollows of rotten tree trunks, under bark, in birds`nests). In MIZ collections, there are voucher specimens for the publication of LüLLwiTz (1916) and the reports of B. BuRAKOwSKI (*) from KFP (the specimen from Rytro is included in the ISEA collection). KFP has not reported occurrence of this species in Upper Silesia (1 specimen from 1881 is deposited in ISEA).

\section{Tenebrio opacus DuFtschмID, 1812}

Pomeranian Lake District: Bielinek nad Odra [VU46], 30 V-1 VI 1983, 2 exx.. leg. BB [MIZ], 23 VII 1992, 10 VI 1999, 4 exx., leg. DK \& LB [ISEA]; Krzymowska Dąbrowa nat. res. [VU57] ad Krzymów. 13 VIII 1993, 1 ex., leg. LB [ISEA]; Szczecin (Stettin) [VV62]. date missing, 1 ex. [MIZ].

Wielkopolska-Kujawy Lowland: Krajkowo [XT38], 4-11 IX 1983, 20 VIII and 11 XI 1984, 5 exx.. leg. DI [USMB]; Rogalin [XT39]. 30 VII 1984, 1 ex.., leg. DI [USMB]; Miszek [CD55]. 13 VIII 1975, 1 ex., leg. JG [USMB].

Western Beskidy Mts.: Krynica [DV97]. VIII 1937, 1 ex., leg. AB [MIZ].

KFP reports its occurrence only in three KFP regions. Data from reviewed collections (coming from regions listed in KFP) confirm the occurrence of this species in western Poland. However, data on habitats of T. opacus (under rotting tree bark and in hollows of broadleaved trees, in old-growth forests and in gardens and single trees) may indicate a possibility of its occurrence in the whole country. Yet it is possible that Poland is crossed by the border of its range (and thus it is not accidental that it was reported only from western KFP regions), and data on its occurrence in Europe should be verified. It is found in central Europe, reaching Greece and Italy in the south, France in the west, and Denmark and Sweden in the north. It has been also recorded from Georgia in the Catalogue of Palaearctic Coleoptera (Lōbl \& Smetana 2008), and, rather generally, from „middle territory of Russia". The most recent monograph on Tenebrionidae in Ukraine does not report occurrence of T. opacus in that country (TSHERNIEY 2005). So it 
is justified to add T. opacus to the Polish Red List, DD category (Paw£owski et al. 2002) and to study more closely its biology and the actual range in Poland.

\section{Alphitobiini ReItTer, 1917}

\section{Alphitobius diaperinus (PANzer, 1797)}

Baltic Coast: Gdynia [CF44], 9 VII 1938. 3 exx.. leg. SS [ISEA].

Wielkopolska-Kujawy Lowland: Rybojedzko [XT19] ad Poznań. 12 VIII 1992, 1 ex.. leg. MA [ISEA]: Puszczykowo [XT29]. 16 VIII 1985. 2 exx., leg. PS [USMB].

Lower Silesia: Wroclaw-Karlowice [XS94]. Czajkowski str.. barracks. 20 VI 1989. 2 exx.. leg. RK [ISEA].

Kraków-Wieluń Upland: Kraków-Salwator [DA14], 21 VIII 2003. 1 ex., leg. JP [ISEA]; Kraków-Śródmieście [DA24]. V 1996, 2 exx.. leg. TW [ISEA].

Roztocze Upland: Florianka [FB30], 1 ex., leg. FF [MIZ].

Western Beskidy Mts.: Polichty [DA91] ad Gromnik, 5 V 1999, 1 ex., leg. AT [ISEA].

Synanthropic and cosmopolitan species. It is extremely difficult to establish which data concern its occurrence in natural conditions on the basis of checklists, catalogs and other summarizing publications. KFP indicates only several locations of reported occurrence of A. diaperinus in Poland, with a comment that it is very rarely found outside storehouses, flour mills and human settlements (usually under loose tree bark). There are no specimens to which publications mentioning the signature of B. BURAKOwSK ${ }^{*}$ refer. It is possible that the data on the occurrence in Gdynia come from the ISEA collection. Interestingly, the number of published records of occurrence of this species outdoor in Poland has remarkably increased in the last decade (inter alia, Kubisz \& Tsinkevich 2001; Greń 2003; Melke \& Grzywocz 2003; Ruta 2007; KLasiński \& Minkina 2008; Gutowski et al. 2010).

\section{Alphitobius laevigatus (FABRICIUs, 1781)}

Baltic Coast: Gdynia [CF44], 2-22 VIII 1938, 12 exx., leg. EM \& AB [MIZ].

Mazowiecka Lowland: Warszawa: Krakowskie Przedmieście [EC08]. 13 III 1915. 3 exx.. leg. TE [MIZ]; Warszawa-Saska Kępa [EC08], 7 V 1916. 1 ex.. leg. TE [MIZ]; Warszawa-Praga [EC08], 21 VIII 1927, 1 ex., leg. TE [MIZ]; „Warszawa”, 27 VI 1906, V 1916, 6 X 1917. 3 exx., leg. TE [MIZ].

Bialowieża Primeval Forest: Bialowieża [FD94], ca. 1880, 1 ex., leg. EW [ISEA].

Świętokrzyskie Mts.: „Świętokrzyskie Mountains”, date missing, 1 ex., leg. TE [MIZ].

Synanthropic and cosmopolitan species, feeding on damp, moldy cereals and its products (a so-called dirty storehouse pest). It is found much more rarely than $A$. diaperinus, which results in a smaller number of faunistic reports thereon. The studied collections include specimens collected in four KFP regions (according to KFP), three of which (Baltic Coast, Bialowieża Primeval Forest a specimen from 1880!, Świętokrzyskie 
Mountains) have not been listed in KFP. All the deposited specimens of $A$. laevigatus date back more than 70 years.

\section{Triboliini GISTEL, 1848}

\section{Latheticus oryzae WATERHOUSE, 1880}

Baltic Coast: Gdynia [CF44]. 9 VIII 1938. 2 exx.. leg. AB [MIZ].

Wielkopolska-Kujawy Lowland: Sieradz [CC41]. VII 1956, 1 ex.. leg. SL [USMB].

Mazowiecka Lowland: Kostrogaj [DD12] ad Plock, storehouse G.S., 25 VI 1955, 1 ex.. leg. SL [MIZ].

The species has not been given a separate number in KFP, i.e., it has not been included into Polish fauna, basing on the opinion that it has been introduced to Poland from abroad and the reports on it are too general. However, it is regarded cosmopolitan, and its occurrence has been documented in many Palearctic regions, including Poland (LỔBL \& SMETANA 2008). In the collections of MIZ and USMB, there are only several specimens of that beetle, but it often happens that species which are common or regarded pests are not properly represented in collections.

\section{Tribolium castaneum (HERBsT, 1797)}

Baltic Coast: Gdynia [CF44], 22 VIII 1938, 1 ex., leg. AB [MIZ].

Pomeranian Lake District: Bielinek nad Odrą [VU46]. 23 VII 1992, 1 ex., leg. DK [ISEA].

Masurian Lake District: Wyskok [EF31] ad Bartoszyce, IX 1991, 1 ex., 1eg. WK [ISEA].

Wielkopolska-Kujawy Lowland: Poznań-Dębina [XU30], 5 VI 1985, 1 ex., leg. MC [ISEA]: Sieradz [CC41], 10 VII 1956. 2 exx.. leg. SL [MIZ].

Mazowiecka Lowland: Warszawa-Śródmieście [DC98], 7 IV 1972. 3 exx., leg. BB [MIZ]; Plock [DD12], 1 VIII 1955. 1 ex., leg. EI [MIZ]: Siennica [EC47], 20 II 1934, 1 ex.. leg. AB [MIZ]; Dęblin [EC51]. 13 X 1925. 1 ex.. leg. HC [MIZ]; „Warszawa”, V 1917. 1 ex.. leg. EI. 19 VII 1956, 1 ex.. leg. SL [MIZ].

Upper Silesia: Taciszów (Tatischau) [CA28], 24 XI 1925, 1 ex.. leg. KI [USMB]; Bytom (Beuthen) [CA58]. 27 I 1931, 1 ex., leg. KI. I 1995, 4 exx., leg. RD [USMB].

Kraków-Wieluń Upland: Kraków [DA 14], 5 X 1899 and 20 II 1904. 7 exx., 1eg. SS [ISEA]; Kraków-Błonia [DA24]. 29 IV 1878. 4 exx.. leg. SS [ISEA]: Kraków-Wesola [DA24], 30 VIII 1900, 5 exx.. leg. MR [ISEA].

Malopolska Upland: Łódź [CC93]. VII 1955, 1 ex.. leg. KP. 15 V 1963, 2 exx., leg. SL [USMB].

Western Beskidy Mts.: Magura Spiska: Czarna Góra [DV47]. 20 X 1990. 1 ex.. leg. TS [ISEA].

A cosmopolitan and synanthropic species. It is a household and storehouse pest, where it feeds on food products; it also damages entomological collections. Found in 
few localities in Poland. Specimens kept in ISEA and USMB collections come from four KFP regions (Masurian Lake District, Kraków-Wieluń Upland, Malopolska Upland, Western Beskidy Mountains) not indicated in KFP. In MIZ collection, there are no specimens connected with the published records bearing B. BuRAKowsKI* signature (Warszawa, Dęblin).

\section{Tribolium confusum JACQUELIN dU VAL, 1862}

Baltic Coast: Gdynia [CF44], 22 VIII 1938, 1 ex., leg. AB [MIZ].

Mazowiecka Lowland: Siennica [EC47]. 1 ex.. leg. AB [MIZ].

Lower Silesia: Legnica (Liegnitz) [WS87]. 1927. 2 exx., coll. USMB; Zloty Stok (Reichenstein) [XR39]. 4 IV 1931, 4 exx., leg. KI [USMB].

Upper Silesia: Bytom (Beuthen) [CA58], 1 X and 7-25 XI 1937, 1 VI 1940, 21 exx., leg. KI [USMB].

Kraków-Wieluń Upland: Kraków [DA14], 5 X 1899, 3 exx., leg. SS [ISEA].

Malopolska Upland: Łódź [CC93], 3 V i 12 XI 1954, 2 exx.. leg. SL [USMB].

Pieniny Mts.: Trzy Korony Mt. [DV57]. 23 V 1972. 1 ex., leg. GB [MIZ].

A synanthropic species, met in whole Europe. Brought to many countries all over the world in food products, on which it feeds. Rarely found outdoors. Reported from Białowieża Primeval Forest in 2001 (KuBISZ \& TsinKEvich 2001). In the reviewed collections, there are specimens from Pieniny Mountains and Upper Silesia (regions not recorded in KFP). In the MIZ collection, there is a voucher specimen for a record signed "B. Burakowski*" (Siennica).

\section{Tribolium destructor UytTenboogaART, 1933}

Baltic Coast: Gdynia [CF44], XII 1956, 2 exx., leg. BS [MIZ].

Wielkopolska-Kujawy Lowland: Poznań [XU30], 26 III 1961, 2 exx., leg. BB [MIZ]. 10 XII 1976. 1 ex.. leg. JG. $5 \mathrm{~V}$ and 6 XII 1982. 3 exx.. leg. LB. 27 XI 1984. 3 exx.. leg. PS [USMB]: Kolo [CC38], 15 I 2000, 1 ex., leg. MK [ISEA].

Mazowiecka Lowland: „Warszawa”, 28 XII 1954, 18 exx., leg. BB [MIZ], 20 VI 1983. 1 ex., leg. TM [ISEA].

Podlasie Lowland: Puszcza Knyszyńska Forest: Rybniki [FE40]. 19 IV 1983, 1 ex.. leg. JG [USMB].

Bialowieża Primeval Forest: Białowieża [FD94]. IV 1990, 3 exx.. leg. DK [ISEA].

Kraków-Wieluń Upland: Kraków-Salwator [DA14], VI 1984, 1 ex., leg. JP [ISEA]; Kraków-Śródmieście [DA24], 13 XI 1983-15 X 1984, 19 exx., leg. MG. 30 VIII 1996. 1 ex.. leg. TW [ISEA].

Malopolska Upland: Łódź [CC93]. 15 V 1963, 5 exx.. leg. SL [USMB].

Sandomierska Lowland: Tarnów [DA94], 4 II 1983, 1 ex., leg. MP [ISEA].

A synanthropic species, feeding on plant and animal products, found in birds' nests (Greń 2003). It is probably widespread everywhere in Poland. Reported in KFP from 6 KFP regions, and then recorded in Bialowieża Primeval Forest (KuBIsz \& Tsinkevich 2001; a voucher specimen is included in the ISEA collection) and in Upper Silesia 
(GREN 2003). In the collections of ISEA and USMB, there are specimens from four further KFP regions (Podlasie Lowland, Kraków-Wielun Upland, Sandomierska Lowland, Małopolska Upland).

\section{Tribolium madens (ChaRPENTIER, 1825)}

Wielkopolska-Kujawy Lowland: Poznań [XU30]. 16 XI 1981, 3 exx.., leg. DI [USMB]; Falmierowo [XU49] ad Wyrzysk. 15 IV 1989. 1 ex.. leg. MJ [ISEA].

Upper Silesia: Bytom [CA58]. 25 VI 1984, 10-12 VI and 2-10 VIII 1987, 5 exx.. leg. MD [USMB]; Zaborze [CA74] ad Oświęcim. 14 V 1901 - 26 VII 1903, 12 exx.. leg. SS [ISEA].

Kraków-Wieluń Upland: Kraków [DAl4]. 29 VII 1920, 1 ex., leg. TE [MIZ]. 7 VII 1920. 1 ex.. leg. SL [USMB].

Malopolska Upland: Łódź [CC93], 15 V 1963, 2 exx.. leg. SL [USMB].

Sandomierska Lowland: Ochmanów [DA33] ad Podłęże, 6 V 1905, 1 ex., leg. SS [ISEA].

A widespread species (Palaearctic and Nearctic), however not regarded cosmopolitan, as three other species of Tribolium genus. A synanthrope (feeding mainly on plant products), also found in natural conditions (under tree bark and in rotten wood of broadleaved trees). According to KFP, that species is rarely recorded in Poland, reported from eight KFP regions. In reviewed collections of ISEA, there is a specimen from 1905 captured in Sandomierska Lowland, and in the USMB collection - found in Malopolska Upland (both faunistic regions not mentioned by KFP). The voucher specimen for the note signed "B. BuRakowski*" (Piaseczno) has not been found in the MIZ collection.

\section{Ulomini BLANCHARD, 1845}

\section{Uloma culinaris (LinNAEUs, 1758)}

Wielkopolska-Kujawy Lowland: Golaszyn [XT12] ad Nowa Sól. 30 VII 1988. 1 ex.. leg. AM [ISEA]: Czeszewo [XT78]. 9 V 1993, 1 ex., leg. LB [ISEA].

Mazowiecka Lowland: Warszawa-Buchnik [DC99]. 7 XI 1954. 1 ex., leg. BB [MIZ]; Warszawa-Bielany [DC99]. 11-13 V 1953, 2 exx., leg. BB [MIZ]; Brzóza [EC21] ad Kozienice. 7 VII 1956, 3 exx., leg. BB [MIZ]; Sewerynów [EC21] ad Kozienice, 7 VII 1956. 2 exx., leg. BB [MIZ]: Celestynów [EC26]. 27 VIII 1949, 4 exx.. leg. ZW, 17 IX 1950, 1 ex.. leg. BB [MIZ]: Urle [ED41]. 9 IV 1905, 2 exx., leg. WM [USMB].

Bialowieża Primeval Forest: Hajnówka [FD74]. 10 VII 1949. 38 exx.. leg. RB [MIZ]; Bialowieski N. P.. f. comp. 340 [FD94]. 3 IV 1966, 6 exx., leg. BB [MIZ]; f. comp. 399 [FD94]. 9 V 1959. 1 ex.. leg. BB [MIZ]: f. comp. 498 [FD94]. 13 VIII 1957. 2 exx.. leg. BB [MIZ]: f. comp. 499 [FD94], 30 VII 1955, 1 ex., leg. BB [MIZ]: Białowieża [FD94], 30 V 1934. 1 ex.. leg. HC. 27 VII 1949. 2 exx.. leg. ZW. 29 IX-5 X 1950. 7 exx.. leg. BB [MIZ]: Dziedzinka [FD94], 2 VII 1992. 1 ex., leg. JG [ISEA]; „Białowieża Primeval Forest at Hwoźna”, 20 III 1952. 1 ex., leg. BB [MIZ]; „Bialowieża Primeval Forest”, 13 VII 1949. 3 exx.. leg. RB [MIZ].

Lower Silesia: Buczyna Szprotawska nat. res. [WT50] ad Przemków, 19 V 2007, 4 exx.. leg. $\mathrm{RD}$ [USMB]. 
Upper Silesia: Rybnik [CA25], 15-16 VII 1934, 5 exx., leg. KL [MIZ]; Katowice-Murcki (Emmanuelssegen) [CA56], 15 VI 1913, 2 exx., leg. GP, 6 VI 1928, 16 IX 1931. 6-9 XI 1935. 18 VI 1936, 56 exx.. leg. KI. 21 V 1930 and 9 XI 1935. 6 exx., leg. HN [USMB]. 30 III 1962. 1 ex.. leg. BB [MIZ]; Bytom (Beuthen) [CA58]. 10 VI 1932 and 1 VI 1934, 4 exx., leg. KI, 10 IV 1936, 2 exx.. leg. HN [USMB]: Zawadzkie [CB21]. 15 X 1950, 2 exx.. leg. BB [MIZ].

Kraków-Wieluń Upland: Miękinia [DA05]. 28 IV 1912. 1 ex.. leg. TE [MIZ]; Krzeszowice [DA05] ad Kraków, 3 X 1976, 2 exx., leg. MB [ISEA].

Lubelska Upland: Pulawy [EB69]. 22 IV 1951, 2 exx.. leg. BB [MIZ]; Bochotnica [EB69] ad Kazimierz Dolny, 28 V 1955. 1 ex.. leg. BB [MIZ].

Roztocze Upland: Zwierzyniec [FB30]. Bukowa Góra, 7 VII 1999. 1 ex.. leg. ST [ISEA]. 3 VII 1910, 3 exx., leg. TE, 26 V 1955, 4 exx., leg. GO \& AR [MIZ]. 3 VI 1987 and 7 VI 1988. 3 exx.. leg. DI [USMB]; Panasówka [FB30]. 7-20 VIII 1911. 3 exx.. leg. TE [MIZ]; Florianka [FB30], date missing. 1 ex., leg. FF [MIZ]; Obrocz [FB41], 21 VI 1912, 1 ex., leg. FF [MIZ]: rez. Nart [FB50], 13-14 VII 1910 and 22 VIII 1912, 6 exx., leg. TE [MIZ], 30 VI 1985 and 9 VII 1999. 3 exx., leg. DK \& ST [ISEA]. 23 IX 1987 and 11 V 1988. 2 exx.. leg. DI [USMB]: Zamość [FB52]. VI 1930, 3 exx., leg. FF [MIZ].

Western Beskidy Mts.: Jastrzębia [DA12], 24 IV 1897, 2 exx.. leg. SS [ISEA]; KrakówSkotniki [DA14]. 22 VII 1954. 3 exx.. leg. RA [USMB]: Rytro [DV78]. 7 VIII 1899 and 23 V 1900, 2 exx.., leg. SS [ISEA], 13 VII 1949, 1 ex., leg. MZ [MIZ]: Łomnica [DV87] ad Piwniczna. 17-18 VII 1898, 1 ex., leg. SS [ISEA].

Eastern Beskidy Mts.: Lesko [EV97]. 10 VII 1969. 1 ex.. coll. USMB; Panieński Czub [FA00] ad Bircza, 22 V 1879, 4 exx., leg. BK [ISEA]: Góra Biala (Splawa) [FA10], 7 VI-6 VII 1884, 8 exx., leg. BK [ISEA]; Krasiczyn [FA11]. 22 IX 1962, 1 ex., leg. BB [MIZ]: Wapowce [FA11], 3 X 1886, 1 ex.. leg. BK [ISEA]: Holubla [FA11], 25-31 V 1884, 3 exx.. leg. BK [ISEA]: Przemyśl-Bakończyce [FA21]. 2 IV 1884, 1 ex., leg. BK [ISEA].

Bieszczady Mts.: Chryszczata Mt. [EV86], 19 VII 1969, 1 ex., leg. BB [MIZ]; Habkowce [EV95], 22 VI-25 VII 1969, 2 exx., leg. BB [MIZ]; Otryt Mt. [FV15], 16 VI 1958, 1 ex., leg. BB [MIZ]: Polana [FV16], 22 VI 1953, 1 ex.. leg. BB [MIZ].

A species widespread all over Europe, reaching western Siberia, and known from Iran. Reported in KFP from 13 regions, but it can be expected that it is spread everywhere in Poland except for higher mountain levels. The reviewed collections contain specimens from two KFP regions which have not been listed in KFP: Kraków-Wieluń Upland and Bieszczady Mountains. There are numerous voucher specimens for notes bearing signature "B. BuRAKowsKI*" in the MIZ collection. The species is quite commonly captured, which is reflected by a large amount of specimens in the reviewed collections, and by numerous publications giving data on its occurrence (inter alia. KuBISZ et al. 2000; Kubisz \& Tsinkevich 2001; Byk \& Byk 2004; Kubisz 2004; Gutowski et al. 2006).

\section{Uloma rufa (Piller \& Mitterpacher, 1783)}

Pomeranian Lake District: Wiele [XV87] ad Karsibór, 11 VIII 2008, 2 exx.. leg. ŁP [ISEA]: Komorz [XV94], 20 IV 1978, 1 ex., leg. JG [USMB].

Masurian Lake District: Łańsk [DE63]. 14 IX-11 X 2003, 5 exx., leg. WJ [ISEA]; Zdunowo [EE42] ad Pisz. 16 IX 1973, 3 exx.. leg. BB [MIZ]: Szeroki Bór [EE44]. 22 X 1962. 
2 exx., leg. BB [MIZ]; f. distr. Topilówka [FE27], Porzecznik, 3 IV 1955, 5 exx.., leg. BB [MIZ]; Bryzgiel [FE38] ad Augustów, 12 VIII 1955, 3 exx., leg. BB [MIZ].

Wielkopolska-Kujawy Lowland: Lipinki Lużyckie [VT92]. 8 VI 1985, 1 ex., leg. LB [USMB]; Nowogród Bobrzański [WT24], 19 IV 1983, 1 ex., leg. LB [USMB]; Miszek [CD55]. 17 VIII 1986. 2 exx., leg. JG [USMB].

Mazowiecka Lowland: Sieraków [DC89]. 1 VII 1953. 1 ex.. leg. BB [MIZ]; Dziekanów Leśny [DC99]. 11 XI 1956, 2 exx., leg. BB [MIZ]; Warszawa-Mlociny [DC99], 25 VIII 1960. 2 exx.. leg. WD [MIZ]; Warszawa-Bielany [DC99], 13 V 1953, 1 ex.. leg. BB [MIZ]; Pomiechówek [DD71]. 7 V 1891. 2 exx.. coll. MIZ: Jabłoń [EC17]. 8 V 1896. 6 exx.. leg. WM [USMB]: Sewerynów [EC21] ad Kozienice, 5 VII 1956, 1 ex., leg. BB [MIZ]; Celestynów [EC26]. 25 VIII 1949, 1 ex., leg. ZW, 14-28 V 1950, 4 exx., leg. BB [MIZ]: Dęblin [EC51], 5 X 1926 and 19 IV 1927. 4 exx., leg. HC [MIZ]: Szumin [ED33] ad Łochów. 18 VIII 1981. 1 ex.. leg. TM [ISEA]: Urle [ED41], 16 VI 1909, 3 exx., leg. WM [USMB]; Zaręby Kościelne [ED74] ad Malkinia, 20 V 1960, 2 exx., leg. IS [MIZ].

Podlasie Lowland: Jata [EC85] ad Łuków, 25 VI 1953, 1 ex.. leg. AR [MIZ]; Choroszczynka [FC65] ad Tuczna. VI 1970, 13 exx., leg. JS [ISEA]; Pilatowszczyzna [FD79], 5-20 VIII 1990, 1 ex.. leg. JP [ISEA]; Puszcza Knyszyńska Forest: ad Rybniki [FE40]. 8 VII 1987, 1 ex.. leg. JG [ISEA]: Kopna Góra [FE60] ad Supraśl. 16 VIII 1985, 1 ex.. leg. DK [ISEA].

Bialowieza Primeval Forest: f. comp. 78A [FD85], 22 IV 1993, 3 exx., leg. J [USMB]; Białowieża [FD94], 29 IX 1950 and 7 IV 1955, 3 exx., leg. BB [MIZ], 17 VIII 1976, 1 ex., leg. MB [ISEA]. 17 XI 1982, 2 exx.. leg. LB [USMB]: f. comp. 399 [FD94]. 9 VI 1959. 1 ex.. leg. BB, 22 IV 1968 and 11 VI 1970, 2 exx.. leg. AD [MIZ]; f. comp. 498 [FD94], 13 VIII 1957 and 28 IX 1968, 4 exx.. leg. BB [MIZ]; f. comp. 499 [FD94], 30 VII 1955, 4 exx., leg. BB [MIZ]: f. comp. 193 [FD95], 31 VII 1965, 1 ex., leg. BB [MIZ]: f. comp. 224 [FD95]. 28 V 1954, 1 ex.. leg. BB [MIZ]; f. comp. 285 [FD95]. 10 V 1959, 1 ex.. leg. BB [MIZ].

Upper Silesia: Rudno (Rauden) [CA18] ad Gliwice, 6 IV, 3 X and 6 XI 1929, 16 exx., leg. HN [USMB]: Błotnica Strzelecka (Blottnitz) [CA19], 31 VIII 1928, 1 ex., leg. HN [USMB]; RybnikParuszowiec (Paruschowitz) [CA25]. 25 VII 1909. 1 ex.. leg. SC [USMB]; Gliwice-Brzezinka (Birkenau) [CA37]. IV 1926, 1 ex., leg. K. Kuntze [USMB]; Szalsza [CA37] ad Tarnowskie Góry. 4 X 1927, l ex., leg. HN [USMB]; Brynek [CA39]. 22 IV and 14-24 X 1928, 12-17 XI 1929. 6 exx., leg. KI [USMB]; Tarnowskie Góry (Tarnowitz) [CA49]. 19 V 1911. 1 ex.. leg. GP [USMB]; Katowice-Panewniki (Pannewitz) [CA56], 25 II 1912, 1 ex., leg. GP [USMB]; Katowice-Murcki (Emmanuelssegen) [CA56], 6 VI and 6 XI 1928, 30 VI 1930, 18 VI 1936, 7 exx., leg. KI [USMB]: Bytom (Beuthen) [CA58]. 1 XI 1922. 1 XII 1927. 20 VI 1940. 3 exx.. leg. KI [USMB]: Grodziec [CA68] ad Będzin. VII 1950. 2 exx., leg. KA [ISEA]; Dąbrowa Górnicza [CA77]. VI 1933, 5 exx.. coll. USMB; Kielcza (Keltsch) [CB20], 10 X 1926. 5 exx.. leg. KI [USMB]; Potempa (Potempa) [CB30] ad Tworóg, 18 X 1937. 9 exx.. leg. HN [USMB]; Tworóg [CB30]. 23 X 1928, 1 ex., leg. HN [USMB]; Lubliniec [CB31], 30 V 1993 and 10-30 IV 1994, 4 exx., leg. RD [USMB].

Kraków-Wieluń Upland: Kraków-Wola Justowska [DA14]. 19 V 1878. 1 ex., leg. SS [ISEA].

Malopolska Upland: Łódź-Lućmierz [CC93]. 18 IV 1965, 1 ex.., leg. KM [USMB]; Spala [DC41], 24 V 1965, 2 exx.. leg. JC [USMB].

Lubelska Upland: Kazimierz Dolny [EB68]. 15 V 1949. 1 ex.. leg. AR [MIZ].

Roztocze Upland: Hamernia [FA48], 6 VIII 1982, 1 ex., leg. KK [USMB]; Czartowe Pole nat. res. [FA49], 14 VIII 1982, 1 ex., leg. DI [USMB]; Zwierzyniec [FB30], 26 V 1955, 3 exx.. leg. AR [MIZ]; Florianka [FB30]. date missing. 1 ex.. leg. FF [MIZ]; Obrocz [FB41]. 7 VI 1955. 2 exx., leg. BB [MIZ]. 
Sandomierska Lowland: Nisko [EA89], 18 IX 1957, 2 exx., leg. BB [MIZ]; Krzeszów [EA98]. VI 1941. 1 ex., leg. FF [MIZ]: Zaleszany [EB61] ad Sandomierz. 23 IV-12 V 1891, 28 exx., leg. SS [ISEA]: Horyniec [FA66], 1-15 VII 1888, 2 exx., leg. SS [ISEA]: Łukawica [FA77] ad Narol, 1 VI 1958, 2 exx., leg. BB [MIZ].

Eastern Sudety Mts.: Polanica-Zdrój [XR08]. 22 IV 1954, l ex., leg. EI [MIZ].

Western Beskidy Mts.: Kraków-Skotniki [DA14]. 27 VII 1954. 2 exx.. leg. RA [USMB]; Mlodów [DV77] ad Rytro, 12 V 1899, 1 ex.. leg. SS [ISEA].

Eastern Beskidy Mts.: Panieński Czub [FA00] ad Bircza. 4 V 1879, 1 ex., leg. BK [ISEA]; Góra Biala (Splawa) [FA10]. 6 VII 1884. 6 exx., leg. BK [ISEA].

Bieszczady Mts.: Habkowce [EV95]. 22 VII 1969. 1 ex., leg. BB [MIZ].

Tatry Mts.: Kuźnice [DV25]. 28 VI 1900, 5 exx., leg. MR [ISEA].

A rather widespread species, similarly as $U$. culinaris, inhabiting moldy wood of broadleaved and coniferous trees, most often rotting tree trunks and stumps of pines, spruces and fir trees. In KFP, it has been reported from eight regions. The reviewed collections contain specimens caught in further six KFP regions: Pomeranian Lake District, Kraków-Wieluń Upland, Małopolska Upland, Lubelska Upland, Eastern Sudety Mts. and Bieszczady Mountains. In the MIZ collections, there are voucher specimens for notes signed "B. Burakowski*". The species is often captured, so there is a large number of specimens in the reviewed entomological collections.

\section{Helopini Latreille, 1802 \\ Nalassus convexus (Comoli, 1837)}

(Fig. 11)

Mazowiecka Lowland: Warszawa-Bielany [DC99], 21 IV 1884. 1 ex.. leg. WM [USMB]; Jabłoń [EC17], 8 V 1896, 1 ex., leg. WM [USMB].

The species has not been reported from Poland so far. It inhabits a small area in the Central Europe (LōBl \& Smetana 2008): Austria, Germany, Liechtenstein, Switzerland, Hungary and Italy, and is considered a mountain species, connected mainly with Alps. It is likely that the specimens in the collection of W. MAcZYńsKI were brought to our country, although Stebnicka (1991) deems its occurrence in Poland possible.

\section{Nalassus dermestoides (ILLIGER, 1798)}

Pomeranian Lake District: rez. Bielinek nad Odrą [VU46], 24 IV 1962, 1 ex.. leg. EK [MIZ], 1 VI 1983, 1 ex., leg. DI [USMB]; Szczecin (Stettin) [VV62], date missing, 1 ex.. coll. MIZ; Szczecin-Warszewo (Stettin. Waldschloss Warsow) [VV62], 9 III 1913, 1 ex., coll. MIZ.

Masu rian Lake District: Puszcza Augustowska Forest: Sajenek [FE36], 30 IV 1990, 1 ex., leg. TW [ISEA].

Wielkopolska-Kujawy Lowland: Lipinki Łużyckie [VT92], 8 VI 1985, 1 ex.. leg. LB [USMB]: Sieciejów [WT02] ad Żary, 20 VIII 1985, 1 ex., leg. PS [USMB]; Nowogród 
Bobrzański [WT24], 13-25 VI 1979. 19 IV 1983, 21 VI 1984, 21 VI 1985, 7 exx., leg. PS \& LB [USMB]: Puszczykowo [XT29], 5 VIII 1985, 1 ex.. leg. PS [USMB]: Międzychód [XT36]. 25 V 1951. 1 ex., leg. RB [MIZ]: Baldoń [CC30] ad Kalisz, 31 III 1983, 1 ex., leg. AM [ISEA]: Kościelec [CC38] ad Kolo, 15 IV 2006, 4 exx., leg. MK [ISEA]; Kolo [CC48], 11 X 1983, 1 ex., leg. TM [ISEA]; Łęczyca [CC76], 28 X 1934, l ex.. leg. AB [MIZ]: Toruń [CD37]. 2 XI 1935. 1 ex.. leg. AB [MIZ]: Ciechocinek [CD56]. 10 VI 1926. 1 ex.. leg. HC [MIZ]: Wloclawek: Kulin nat. res. [CD63]; 3 V 1951, 1 ex., leg. RB [MIZ]; Szpetal Dolny [CD63] ad Wloclawek. 28 V 1954, 1 ex.., leg. AR [MIZ].

Mazowiecka Lowland: Lasy Młochowskie Forest: Nowa Wieś [DC77]. 30 VIII 1950. 1 ex.. leg. RB [MIZ]; Podkowa Leśna [DC87], 15 XII 1929 and 6 X 1934, 10 exx.. leg. TE. 5 XII 1948. 2 exx., leg. MZ [MIZ]; Puszcza Kampinoska Forest: Sieraków [DC89], 27 I 1956, 1 ex., leg. BB [MIZ]: Łoś [DC95] ad Grójec. 6 XII 1956, 1 ex.. leg. BB [MIZ]: Lomianki [DC99]. 29 X 1928. 1 ex.. leg. TE [MIZ]; Dziekanów Leśny [DC99]. 30 IX 1951 and 4 XII 1955, 5 exx., leg. BB [MIZ]: Warszawa-Buchnik [DC99]. 7 XI 1954. 2 exx., leg. BB [MIZ]; Warszawa-Mlociny [DC99], 28 V 1918. 2 exx.. leg. TE. 25 X 1953. 1 ex., leg. BB [MIZ]: Warszawa-Bielany [DC99]. 9 XI 1921. 1 ex., leg. HC, 29 III 1934, 1 ex., leg. TE, 15 VII 1950, 22 VI 1952, 11 V 1953, 4 II 1958, 13 IX 1962, 30 V 1963, 27 II 1966, 7 exx., leg. BB [MIZ]; Pomiechówek [DD71]. 30 X 1927 and 7 IV 1928. 2 exx., leg. AB, 22 VIII and 22 X 1950, 2 exx., leg. BB. 22 III 1951. 1 ex.. leg. RB [MIZ]: Jabłonna [DD90], 2 IV 1893, 1 ex.. leg. WM [USMB]; Zalesie [EC06] ad Piaseczno, 23 IV 1930. 1 ex., leg. TE, 20 IV 1947. 1 ex., leg. WR [MIZ]; Żabieniec [EC06] ad Piaseczno, 12 VI 1952. 1 ex.. leg. WE [ISEA]; Warszawa-Powsin [EC07]. 17 VI 1954. 1 ex.. leg. BB [MIZ]; Skolimów [EC07]. 14 XI 1935 and 14 IX 1939. 41 exx., leg. TE [MIZ]; Pustelnik [EC09]. 1 IV 1924. 2 exx., leg. TE [MIZ]; Drewnica [EC09], 4 IX 1920, 1 ex., leg. TE [MIZ]; Jabloń [EC17], 1 VII 1901, 1 ex.. leg. WM [USMB]: Otwock [EC17], 15 V 1895, 1 ex.. leg. WM [USMB]: WarszawaAnin [EC18]. 10 VI 1947. 2-9 I and 30 XI 1949, 9 exx., leg. KG. 6 IX 1947. 2 exx.. leg. WR. 23-26 IX 1947, 2 exx., leg. RB, V 1949, 1 ex., leg. WD [MIZ]; Warszawa-Wawer [EC18], 14 IV 1897, 3 exx., leg. WM [USMB]. 27 II 1922, 1 ex., leg. HC, 27 IX 1927, 20 exx., leg. TE, 21 IV 1960. 12 X 1961. 10 V 1970, 4 exx.. leg. BB [MIZ]; Żwir [EC18]. 26 IX 1950 and 8 XI 1951. 2 exx., leg. BR [MIZ]; Celestynów [EC26], 11 X 1931, 9 exx., leg. SS [ISEA], 10 IV 1949, 1 ex.. leg. WR. 9 IV 1951. 2 exx., leg. RB \& MZ [MIZ]; Dębe Wielkie [EC38], 8 VII 1916, 1 ex.. leg. TE [MIZ]: Dęblin [EC51]. 16 IV 1927 and 14 IV 1936. 3 exx.. leg. HC [MIZ]; Czarna Struga [ED00], 6 X 1927, 2 exx., leg. TE [MIZ]; Kalinowo [ED65], 6 VII 1933 and 3 VIII 1935. 2 exx.. leg. AB [MIZ]: „Warszawa”, 4 VI 1982, 1 ex., leg. WM [USMB].

Podlasie Lowland: Jata nat. res. [EC85] ad Łuków, 23 XI 1958, 1 ex., leg. BB [MIZ].

Bialowieża Primeval Forest: Orzeszkowo [FD74], 11 III 1997, 1 ex., leg. JL [USMB]; Hajnówka [FD74], 7 VII 1930. 1 ex.. leg. TE [MIZ].

Lower Silesia: Ślęża Mt. [XS23], 22 IV 1957. 2 exx., leg. BB [MIZ]; ad Chocianów [WS69]: former forest div. Trzmiel, f. distr. Balanów, X-XI 1953, 1 ex., leg. SA [ISEA].

Upper Silesia: Rybnik-Paruszowiec (Paruschowitz) [CA25], 24 VI 1911. 1 ex.. leg. SC [USMB]; Katowice-Murcki (Emmanuelssegen) [CA56]. 17 XI 1938, 1 ex., leg. HN [USMB]; Chełmek [CA75], 26-31 III 1883, 2 exx., leg. SS [ISEA]: Babice: Lipowiec castle [CA84], 20 IV 1882, 1 ex., leg. SS [ISEA]; Żarki [CA84] ad Chrzanów, 24 IV 1881, 2 exx., leg. SS [ISEA]: Pogorzyce [CA85] ad Libiąż. 8 VI 1889. 1 ex.. leg. SS [ISEA]: Kielcza (Keltsch) [CB20]. 10 X 1926, 1 ex.. leg. KI [USMB]: Zawadzkie [CB20]. 14 X 1927. 1 ex.. leg. KI [USMB]: Potempa [CB30] ad Tworóg. 18 X 1937, 1 ex., leg. HN [USMB].

Kraków-Wieluń Upland: Kraków [DA14]. ca. 1900, 5 exx., leg. MR [ISEA]; Ojców [DA16], 25 IV 1952, 1 ex., leg. MZ [MIZ]. 
Malopolska Upland: Pabianice [CC82], 13 IV 1914, 2 exx., leg. EI [MIZ]; Marchocice [DA47] ad Miechów. $30 \mathrm{~V}$ 1954, 1 ex.. leg. WS [ISEA]: Pińczów [DA69]. 6 VI 1991. 1 ex.. leg. MM [ISEA]; Malkowice [DA76], 31 V 1991, 1 ex., leg. MM [ISEA]; Skorocice [DA78]. 10 IX 1963. 1 ex.. leg. BB [MIZ]: Kików [DA98]. 3 IV 1991. 1 ex.. leg. JP [ISEA]: Murawy Dobromierskie nat. res. [DB26] ad Radomsko, $11 \mathrm{~V}$ 1994, 1 ex., leg. DK [ISEA]; Skowronno [DB60]. 25 V 1963, 30 IX 1967, 19 IX 1979, 3 IV 1990, 8 exx., leg. WS \& MM [ISEA], 18 VI 1995 and 15 VII 1996. 2 exx.. leg. RD [USMB]: Spalski Landscape Park [DC31]. 12-13 V 1999. 1 ex., leg. RD [USMB]; Dwikozy [EB52] ad Sandomierz, 21 V 1955, 2 exx.., leg. BB [MIZ], 31 V 1967, 1 ex., leg. WS [ISEA].

Świętokrzyskie Mts.: Miedzianka [DB53] ad Kielce, 11 VI 1984, 1 ex.. leg. WJ [MIZ].

Lubelska Upland: Opole Lubelskie [EB66]. 3 VI 1915, 1 ex., leg. EI [MIZ]; Kazimierz Dolny [EB68], 15 VI 1962 and 14 V 1963, 2 exx.. leg. WS [ISEA]; Pulawy [EB69], 21 IV-8 V 1925, 3 exx.. leg. HC. 2 X 1949. 1 ex.. leg. RB [MIZ]: Bochotnica [EB69] ad Pulawy, 16 VI 1962, 1 ex., leg. WS [ISEA].

Roztocze Upland: Ulów [FA58]. 13 VII 1919. 2 exx.. leg. TE [MIZ]; Szarowola [FA69]. 20 VII 1912. 1 ex.. leg. TE [MIZ].

Sandomierska Lowland: Klaj [DA53]. 24 V 1901. 1904. 15 XI 1908. 22 exx., leg. MR \& SS [ISEA]; Smęgorzów [EA06] ad Dąbrowa Tarnowska, 15 VII-30 VIII 1897. 1 ex.. leg. SS [ISEA]; Kochany [EB81] ad Stalowa Wola. X-XI 1953, 2 exx., leg. SA [ISEA]; Jaroslaw [FA24], 1 IX 1887, 1 ex., leg. SS [ISEA]; Ruda Różaniecka [FA57] ad Lubaczów. X-XI 1953. 2 exx.. leg. SA [ISEA].

Western Sudety Mts.: Karkonoski N. P., shelter-house „Strzecha Akademicka” [WS52], 23 V 1973, 1 ex., leg. NI [ISEA].

Western Beskidy Mts.: Kraków-Borek Falęcki [DA23], IV-V 1874, 2 exx., leg. BK [ISEA]; Wieliczka: Las Krzyszkowicki forest [DA23]. 8 VI 1879 i 29 V-5 VI 1880, 4 exx., leg. SS [ISEA].

Eastern Beskidy Mts.: Makowa [FA20] ad Przemyś1, 8 V 1990, 2 exx.. leg. JM [ISEA].

A species from the Central Europe, found mainly on pine trees (BurAKowski et al. 1987) or near oak trunks (RUTA 2007). KFP gives reports virtually from all regions, so it is just a formality to confirm that the reviewed collections contain specimens captured in further four KFP regions: Podlasie Lowland, Lower Silesia, Świętokrzyskie Mountains, Western Beskidy Mountains. The species is often captured, and the collections include numerous specimens (there are voucher specimens for records signed "B. BuraKowsKi*" in MIZ collections).

\section{Nalassus laevioctostriatus (GoEze, 1777)}

Wielkopolska-Kujawy Lowland: Nowogród Bobrzański [WT13], 6 IX 1982. 2 exx., leg. PS [ISEA]; Łęczyca [CC76], 24 V 1937. 1 ex.. leg. AB [MIZ].

The species inhabits mainly western and central Europe. It is regarded a western vicariant of $N$. dermestoides (BURAKOWSKI et al. 1987), and sometimes it is synonymized with it (NovAK 2005). However, we share the point of view of Catalogue of Palaearctic Coleoptera (LöвL \& SMETANa 2008), where both taxa are given as separate species. 
Found in broadleaved trees. In the reviewed collections, there are several specimens captured in Wielkopolska-Kujawy Lowland (not listed in KFP).

\section{Stenomax aeneus (ScopoLI, 1763)}

Masurian Lake District: Puszcza Augustowska Forest: ad Plaska [FE47]. 26 VI 1986. 1 ex., leg. JG [ISEA].

Wielkopolska-Kujawy Lowland: Biecz (Beitsch) [VT83], 1-5 V 1944. 2 exx., coll. USMB.

Mazowiecka Lowland: Świder [EC17], 15 VII 1900, 1 ex., coll. MIZ; Kozienice [EC31]. 23 IV 1986. 1 ex.. leg. RZ [ISEA]: Klembów [ED20]. 6 V 1962, 1 ex.. leg. BB [MIZ]: Biel [ED64], date missing. 1 ex.. leg. KN [USMB].

Podlasie Lowland: Międzyrzec Podlaski [FC26], date missing. 2 exx.. leg. EI [MIZ]; Biala Podlaska [FC46], 30 VII 1933, 1 ex.. leg. AD [MIZ].

Bialowieża Primeval Forest: f. comp. 632 [FD73], 12 V 1990, 1 ex.. leg. BB [MIZ]; Hajnówka [FD74]. 4 V 1977. 1 ex.. leg. MB [ISEA]: f. comp. 523B [FD83]. 25 V 1980. 2 exx.. leg. JG [USMB]; f. comp. 417 [FD84], 13 V 1961, 1 ex.. leg. AD [MIZ]: f. comp. 80C [FD85]. 23 VII 1981. 1 ex., leg. JL [USMB]; f. comp. 156 [FD85]. 26 VI 1991, 1 ex., leg. TM [ISEA]: f. comp. 369 [FD94]. 17 IV 1967. 1 ex.. leg. BB [MIZ]: f. comp. 474 [FD94]. 16 V 1969. 1 ex.. leg. BB [MIZ]; Białowieża [FD94]. 13 V 1985, 1 ex., leg. JG [ISEA], 16 V 1981, 1 ex., leg. LB [USMB].

Upper Silesia: Tworków [CA04] ad Racibórz. 30 IV 1950. 1 ex., coll. USMB; Racibórz (Ratibor) [CA05], 5 VI 1910, 1 ex., coll. USMB: Pogrzebień [CA05] ad Racibórz, 20 V 1935. 2 exx.. leg. WY [USMB]; Boguszowice (Boguschowitz) [CA24]. 20 V 1918. 1 ex.. coll. USMB.

Kraków-Wieluń Upland: Wręczyca [CB53]. 17 V 1923, 1 ex., leg. KR [MIZ]; Zakrzew [CB54] ad Kłobuck. 7 VI 1924, 1 ex., leg. HC [MIZ]; Zloty Potok [CB81]. 6 V 1928, 2 exx., leg. JF [MIZ]: Mirów [CB90]. 12 VI 1896, 1 ex.. leg. WM [USMB].

Malopolska Upland: Jaksice [DA18] ad Miechów. 31 V 1991, 1 ex.. leg. JM [ISEA]; Stary Wiśnicz [DA62]. 15-29 V 1912, 32 exx.. leg. SS [ISEA]; Krzyżanowice [DA68] ad Pińczów. 23 V 1953, 1 ex.. leg. BB [MIZ], 16 VI 1965, lex.. leg. WS [ISEA]; Bogucice [DA79] ad Pińczów. 14 V 1964, 1 ex.. leg. WS [ISEA]: Dwikozy [EB52], 21 V 1955, 1 ex... leg. BB [MIZ].

Świętokrzyskie Mts.: Łysa Góra Mt. [EB03], 2 VI 1956, 1 ex.., leg. BB [MIZ].

Lubelska Upland: Kazimierz Dolny [EB68], 30 V 1948, 1 ex.., leg. RB, 27 V 1955, 1 ex.. leg. BB [MIZ]. 12 VI 2003, 1 ex.. leg. EN [ISEA]: Puławy [EB69]. 17-29 IV 1925. 3 exx.. leg. HC [MIZ]: Gródek [GB01] ad Hrubieszów. 24 V 1985, 1 ex., leg. MM [ISEA].

Roztocze Upland: Justynówka [FA79] ad Tomaszów Lubelski. 23 IV 1986, 1 ex.. leg. LB [USMB]: Zwierzyniec [FB40], 6 VI 1987, 2 exx.. leg. DI [USMB]: Obrocz [FB41]. 7 VI 1955. 1 ex.. leg. BB [MIZ]; Ulica [FB50] ad Suchowola, 21 V 1985, 1 ex., leg. MM [ISEA]: NartCzerkies nat. res. [FB50], 11 V 1988, 6 exx.. leg. DI [USMB], 15 V 1987, 6 VI 1990, 2 exx.. leg. DK \& JG [ISEA].

Sandomierska Lowland: Puszcza Niepolomicka Forest: Kłaj [DA53], 1904, 27 exx., leg. MR. 9 IV 1936. 16 IV 1937 and 13 IV 1939. 9 exx.. leg. SP. 13 V 1933. 1 ex.. leg. SS [ISEA]: Stanislawice [DA53]. 18 IV 1986, 1 ex.. leg. DK [ISEA]; Zaleszany [EB61] ad Sandomierz, 1 V 1889. 1 ex.. leg. SS [ISEA]; Pawłosiów [FA13] ad Jarosław. 5 VI and $30 \mathrm{~V}$ 1889. 4 exx.. leg. SS [ISEA]: Jaroslaw [FA24], 9 V 1889, 1 ex.. leg. SS [ISEA]: Janów Lubelski [FB01], 9 VII 1974. 1 ex., leg. MG [ISEA]. 
Western Beskidy Mts.: Cieszyn (Teschen) [CA21]. date missing, 1 ex.. leg. VW [MIZ]; Zawada [DA22] ad Myślenice, 27 IV 1900, 1 ex., leg. SS [ISEA]; Kraków-Soboniowice [DA23]. 28 V 1898, 1 ex.. leg. SS [ISEA]; Wieliczka: Las Krzyszkowicki forest [DA23]. 8 VI 1879. 2 exx., leg. SS [ISEA]; Głogoczów [DA23], 28 V-5 VI 1897, 1 IV 1898 and 1 VI 1899, 15 exx.. leg. SS [ISEA]: Mogilany [DA23], 28 IV 1898, 1 ex.. leg. SS [ISEA]: Dziekanowice [DA32]. 29 V 1965. 1 ex.. coll. ISEA: Tęgoborza [DA70]. 10-30 V 1892. 1-13 VI 1892. 8 exx.. leg. SS [ISEA]: Roztoka [DA82] ad Zakliczyn, 30 IV-2 V 1987, 2 exx., leg. DK \& RK [ISEA]; Polichty [DA91] ad Gromnik. 6 VI 1998, 12 IV 1999. 2 exx., leg. DK [ISEA]: Młodów [DV77] ad Rytro. 12 V 1892. 6 exx.. leg. SS [ISEA]: Rytro [DV78]. 8 V-10 VIII 1892. 16 V-21 VI 1896. 11 V-11 VIII 1899. 20 exx., leg. SS [ISEA]; Nowy Sącz-Goląbkowice [DV79]. 15 V 1892, 26 IV-1 V 1920, 3 exx., leg. SS [ISEA]; Chełmiec [DV79], 25 V 1892, 1 ex.. leg. SS [ISEA]; Żegiestów [DV87]. 15 VIII 1892. 1 ex.. leg. SS [ISEA]: Ptaszkowa [DV99] ad Grybów, 5 VI 1892 and 30 VI 1896, 2 exx.. leg. SS [ISEA].

Eastern Beskidy Mts.: Sokól [EV19] ad Gorlice, 1 V 1995, 1 ex., leg. RZ [ISEA]; Łukawica [EV98] ad Lesko. 1 VI 1958, 3 exx.. leg. RB [MIZ]: Itna [EV99] ad Mrzyglód. 10-11 V 1879. 21-22 V 1881, 2 exx., leg. BK [ISEA]; Bircza [FA00]. 17 V 1989. 1 ex., leg. MM [ISEA]: Panieński Czub [FA00] ad Bircza, 4-22 V 1879, 3 exx.. leg. BK [ISEA]; Góra Biala (Splawa) [FA10]. 7 VI-6 VII 1884, 2 exx.. leg. BK [ISEA]: Holubla [FA11]. 25-31 V 1884 and $17 \mathrm{~V}$ 1879, 7 exx., leg. BK [ISEA]; Kaczmarowa Mt. [FA21] ad Łętownia, 28 IV 1879, 3 exx., leg. BK [ISEA]; Przemyśl: Ostrów [FA21]. 31 V 1879 and 26 V-3 VII 1881, 6 exx.., leg. BK [ISEA]: Przemyśl-Budy [FA21]. VI-VII 1881. 1 ex.. leg. BK [ISEA]; Przemyśl-Lipowica [FA21]. 2 VII 1884 and VI 1886, 4 exx., leg. BK [ISEA]: Przemyśl-Pralkowce [FA21]. 27 IV 1879, 8 V 1883. 3 exx.. leg. BK [ISEA]; Łętownia [FA21] ad Przemyśl, $10 \mathrm{~V}$ 1884, 2 exx.. leg. BK [ISEA].

Bieszczady Mts.: Baligród [EV96]. 28 VII 1954, 1 ex., leg. TO [MIZ].

Pieniny Mts.: Dlugi Gronik [DV57]. 21 VI 1973, 1 ex., leg. GB [MIZ].

The data on occurrence of Stenomax lanipes (L.) in KFP (index number 3996) also apply to $S$. aeneus, as both names have been synonymized (LôBL \& SMETANa 2008). For this reason, it has been reported together from 13 KFP regions. In the reviewed collections, there are specimens from four other KFP regions: Wielkopolska-Kujawy Lowland, Podlasie Lowland, Western Beskidy Mountains, Bieszczady Mountains. The beetles are quite commonly caught, which results in high number of specimens deposited in the collections (MIZ includes numerous voucher specimens for notes signed "B. BuRAKOWSKI*").

\section{Blaptini LeACH, 1815}

\section{Blaps halophila Fischer von WALDHEIM, 1822}

Lubelska Upland: Gródek [GB03] ad Hrubieszów, 10 VII 1962 - 5 V 1965. 5 exx.. leg. WS. 10 VII 1970 - 6 VII 1971, 3 exx.. leg. ZS [ISEA].

A Ponto-Siberian species; the western border of its range crosses the south-eastern Poland, where some localities were found only near Hrubieszów (BuRAKowski et al. 1987). The beetle does not inhabit houses, it lives in xerothermic hillsides, fields and 
wastelands. In ISEA collection, there are voucher specimens for the works of W. SZYMCZakowsis $(1965,1973)$. It is listed in the Polish Red List, VU category (Pawlowski et al. 2002), as is the case of the remaining species of Blaps genus.

\section{Blaps lethifera lethifera MARSHAM, 1802}

Wielkopolska-Kujawy Lowland: Rawicz (Rawitsch) [XT22]. 1903. 1 ex.. leg. FK [MIZ].

Upper Silesia: Racibórz (Ratibor) [CA05], 6 X 1911, 1 ex., coll. USMB; Oświęcim [CA74]. 11 VIII 1931. 1 ex., leg. KB [MIZ].

A synanthropic or wild species, widespread all over Europe, from northern Africa to southern Scandinavia. Known in Poland from few localities of 10 KFP regions (BurAKOwSKI et al. 1987) only as a synanthrope; inhabits damp and dark but not cold outbuildings. Listed in the Polish Red List, EN category (Pawlowski et al. 2002). Only one of its subspecies, B. lethifera lethifera Marsiam, 1802, is found in Poland; B. lethifera pterotapha FISCHER VON WALDHEIM, 1832 is a Caucasian subspecies, and B. lethifera subalpina MÉNÉTRIÉs, 1832 is an endemic subspecies met in the south of European part of Russia.

\section{Blaps mortisaga (Linnaeus, 1758)}

Pomeranian Lake District: Koszalin (Coeslin) [WA70], date missing, 5 exx., leg. AL [MIZ].

Masurian Lake District: Augustów [FE36]. 28 VI-22 VII 1936. 1 ex.. leg. EM [MIZ].

Wielkopolska-Kujawy Lowland: Biecz (Beitsch) [VT83]. 8 XII 1948, 1 ex.. coll. USMB; Nowa Sól (Neusalz) [WT43]. IV 1906, 2 exx.. coll. MIZ; Mlodzikowo [XT57] ad Solec Wielkopolski. 28 IX 1983, 2 exx.. leg. MA [USMB]: Stobnica [XU04] ad Obrzycko, 30 VIII 1980 and 15 X 1982. 2 exx.. leg. JA [USMB]: Sycyn Dolny [XU13] ad Szamotuly, 24 IV 1994. 2 exx.. leg. MA [ISEA]; Luboń [XU20], date missing. 1 ex.. leg. WY [USMB]; Poznań [XU21], date missing. 1 ex.. coll. MIZ: Łęczyca [CC76]. VII 1937. 2 exx.. leg. AB [MIZ]: Silno [CD46]. 8 IV 1977, 1 ex., leg. BA [MIZ]; Miszek [CD65], 10 III 1976, 1 ex., leg. JG [MIZ].

Mazowiecka Lowland: Łomianki [DC99]. 24 VII 1910. 1 ex.. leg. TE [MIZ]; WarszawaRuda Marymoncka [DC99]. 1895. 1 ex.. leg. TE [MIZ]; Pomiechówek [DD71], 27 IV 1928 and 7 III 1933, 2 exx.., leg. AB [MIZ]: Piaseczno [EC06], 6 V 1923. 1 ex.., leg. HC [MIZ]: Sulejówek [EC18], 26 V 1957. 5 exx., leg. WE [MIZ]: Dębe Wielkie [EC38]. 18 IV 1916, 1 ex.. leg. TE [MIZ]: Siennica [EC47]. 22 V 1933 and 20 IV 1934. 2 exx., leg. AB [MIZ]: Dęblin [EC51]. 2 VIII 1933, 1 ex.. leg. HC [MIZ]: Zegrze [ED01]. 15 V 1937, 1 ex.., leg. TE [MIZ] ; „Warszawa”. 20 II 1919, 1 ex., leg. TE [MIZ].

Podlasie Lowland: Międzyrzec Podlaski [FC26], date missing. 1 ex.. leg. EI [MIZ].

Upper Silesia: Pogrzebień [CA05] ad Racibórz, 7 X 1931, 3 exx.., leg. WY [USMB]; Rybnik-Paruszowiec (Paruschowitz) [CA25]. 8 III 1911. 4 exx.. leg. SC [USMB]; Zabrze-Maciejów (Mathesdorf) [CA37]. 1932, 1 ex., leg. GF [USMB]; Ruda Śląska (Ruda) [CA47]. 7 VIII 1923. 1 ex.. coll. USMB. 5 I 1926, 2 exx.. leg. Hr. Franciszek StriEcke. 29 XII 1925. 18 VIII 1928. 15 IX 1929. 5 exx., leg. WU [USMB]: Zabrze (Hindenburg O/S) [CA47]. 1 X 1928, 1 ex.. leg. KI [USMB]; Tarnowskie Góry (Tarnowitz) [CA49]. date missing. 2 exx., leg. KT [USMB]; Ruda Śląska-Kochlowice [CA57], 27 V 1965, 1 ex.. leg. KW [USMB]; Bytom (Beuthen) [CA58], 8 VII 
1924 and 3 VI 1931, 2 exx., leg. KI [USMB]: Chelmek [CA75], 16 VII 1876, 16 VII 1878, 10-20 III 1881. VI 1888, 4 exx., leg. SS [ISEA]; Jaworzno-Szczakowa [CA76]. VII-X 1910, 4 exx., leg. SP [ISEA]: Daborowa Górnicza [CA77]. VII 1933. 1 ex., coll. USMB; Byczyna [CB06]. 1957 and 25 IX 1960, 2 exx., leg. SI [USMB]; Truskolasy [CB43], 12 VI 1938, 1 ex., coll. MIZ.

Kraków-Wieluń Upland: Opatów [CB44], 23 X-4 XI 1913, 2 exx.. leg. EI [MIZ]; Czatkowice [DA05] ad Krzeszowice. 26 V 1914. 1 ex.. leg. SS [ISEA]: Kraków [DA14]. 23 VI 1911. 1 ex.. leg. TE [MIZ], 25 VII and 7 IX 1936, 3 exx., leg. SS \& SP [ISEA]: Kraków: Botanical Garden [DA24]. ca. 1900. 2 exx., leg. MR [ISEA]; Kraków-Podgórze [DA24], 1912, 4 exx.. leg. SP [ISEA].

Malopolska Upland: Krzyżanowice [DA68]. 15 VII 1953, 1 ex.. leg. BB [MIZ]; Zacharzów [DC91]. VII 1932, 1 ex., leg. GR [MIZ]: Sokolniki [EB51] ad Sandomierz. VIII 1991. 1 ex., leg. KC [ISEA].

Lubelska Upland: Pulawy [EB69]. 19 IV 1925. 1 ex.. leg. HC [MIZ].

Roztocze Upland: Florianka [FB30], 18 XI 1905 and 28 VI 1914, 3 exx., leg. FF [MIZ]; Zamość [FB52], VI 1930, 1 ex., leg. FF [MIZ].

Sandomierska Lowland: Podłęże [DA44]. 1913. 2 exx.. leg. EM [MIZ]; Wierzchoslawice [DA94] ad Tarnów, IX 1918, 1 ex., leg. SS [ISEA]: Ćwików [DA96] ad Olesno, 24 VIII 1984. 1 ex., leg. T. Nowak [ISEA]; Smęgorzów [EA06] ad Dąbrowa Tarnowska, 17 VII-30 VIII 1897. 5 exx.. leg. SS [ISEA]; Dąbrowica [EA07] ad Szczucin. 11 VIII 1894, 17 exx.. leg. SS [ISEA]; Padew Narodowa [EA38], 19 III 1900, 1 ex., leg. SS [ISEA]; Zaleszany [EB61] ad Sandomierz. $1 \mathrm{~V} 1891,1$ ex., leg. SS [ISEA].

Western Beskidy Mts.: Bielsko-Biala [CA51], date missing. 1 ex.. leg. TE [MIZ]; Żywiec [CA60], 1894, 1 ex., leg. SS [ISEA]; Czaniec [CA72] ad Kęty, 24 VII 1910, 1ex.. SS [ISEA]: Slomiróg [DA43]. V 1912. 2 exx., leg. EM [MIZ]; Bochnia [DA53]. 19 VIII-5 IX 1938, 7 exx., leg. EM [MIZ]; Piwniczna [DV77]. 8 VII 1898, 1 ex., leg. SS [ISEA]; Rytro [DV78]. 14 VII 1899. 1 ex.. leg. SS [ISEA].

Nowotarska Dale: Czarny Dunajec [DV17], VIII 1925, 1 ex., leg. SS [ISEA].

Eastern Beskidy Mts.: Przemyśl: Wzniesienie [FA21], IX 1886. 1 ex.. leg. BK [ISEA].

Pieniny Mts.: Krościenko [DV57], 9-21 VIII 1928. 2 exx., leg. TE [MIZ].

A species widespread in all central and southern Europe as a synanthrope, while in eastern Europe, wild populations occur. RuTA (2005) showed occurrence in Poland of a small population (probably coming from human settlements) living in natural conditions in the valley of the Noteć River. In the reviewed collections, there are specimens collected in 1876-1994, however mainly in the beginning of the $20^{\text {th }}$ century. KFP reports occurrence of this species in 13 regions, and data on labels of studied beetles indicate 5 further KFP regions: Podlasie Lowland, Małopolska Upland (also listed by RuTA in his work from 2005), Lubelska Upland, Nowotarska Dale and Pieniny Mts.. B. mortisaga is listed in Polish Red List, EN category (PAwlowski et al. 2002) and in Polish Red Data Book of Animals (Konwerski 2004).

\section{Blaps mucronata LATREILLE, 1804}

Masurian Lake District: Bryzgiel [FE38]. 11 VIII 1955. 1 ex.. leg. BB [MIZ].

Upper Silesia: Jaworzno-Szczakowa [CA76]. VII 1935, 3 exx., coll. MIZ; Dąbrowa Górnicza [CA77], VIII 1936, 1 ex., coll. USMB.

Malopolska Upland: Krzyżanowice [DA68]. 12 V 1953. 1 ex.. leg. MZ [MIZ]. 
A synanthropic species, extremely rarely met in Poland. In KFP, it is reported from single locations in five regions. Data on labels of specimens deposited in MIZ and USMB confirm its occurrence in Masurian Lake District (B. BuRAKowski*) and in Upper Silesia, and add Malopolska Upland (not listed in KFP). The species is listed in the Polish Red List, EN category (PAwLOwski et al. 2002).

\section{Pedinini Eschscholtz, 1829}

\section{Phylan gibbus (FABRICIUS, 1775)}

Baltic Coast: Wolin-Wiselka [VV78]. 19 VIII 1997, 6 exx., leg. RD [USMB]; Pobierowo [VV98] ad Kamień Pomorski. 21 V-5 VI 1960. 1 ex.. leg. JP [ISEA]. 20 VI 1955 and 19 VI 1965. 3 exx., leg. BB [MIZ]; Mrzeżyno [WA10] ad Trzebiatów, 3 VIII 1973, 1 ex.. leg. MI [ISEA]; Dźwirzyno [WA20]. 9 VII 1980. 1 ex.. leg. TM [ISEA]. 7 VI 1989. 1 ex.. leg. RD [USMB]: Ustronie Morskie [WA40], 10 VIII 1953, 2 exx.., leg. SJ [MIZ]; Sarbinowo [WA61], 8-18 VIII 1995. 1 ex.. leg. JB [USMB]: Jarosławiec [WA94]. 16-23 VII 1955. 2 exx., leg. MZ [MIZ]: Karwia [CF27], 10-16 VIII 1934, 8 exx... leg. EM. 25 VIII 1958 and 10 VIII 1963, 5 exx., leg. RB. 17 VIII 1967. 2 exx.. leg. BB [MIZ]. 18 VI 1996. 8 exx.. leg. RD [USMB]: Jastrzębia Góra [CF28]. 16-24 VI 1996, 5 exx., leg. RD [USMB]: Wladyslawowo [CF37], 18 VI 1993, 3 exx., leg. RD [USMB]; Gdańsk [CF42]. date missing, 1 ex., leg. Bohnsack [ISEA]; Gdańsk-Brzeźno [CF43]. 7-10 VI 1948, 2 exx.. leg. RB [MIZ]; Sopot [CF43]. 2 VI 1987. 1 ex.. leg. RK [ISEA]: Gdynia [CF44]. 25 VII 1927 and 2 VIII 1931, 8 exx.., leg. SS [ISEA]: Jastarnia [CF46]. 3 VIII 1931, 20 exx.. leg. SS [ISEA]. 30 V 1978, 1 ex., leg. JG. 22 VI 1996, 1 ex.. leg. RD [USMB]: Hel [CF55]. 22 VII 1927. 6 exx., leg. SS [ISEA]: Jurata [CF56]. 2-12 VI 1972, 8 exx., leg. ZS [ISEA]: Łysica [CF92] ad Elblag. 8 VIII 1960. 7 exx.. leg. BB [MIZ]: Piaski [DF03] ad Krynica Morska. 28 VII 1978, 9 exx., leg. BB [MIZ]; Mierzeja Wiślana (Frische Nehrung), date missing. 3 exx., leg. KN [MIZ. USMB]; „Pomorze”. date missing. 24 exx.. leg. EM [MIZ].

Pomeranian Lake District: Szczecin (Stettin) [VV62], date missing. 4 exx., coll. MIZ; Koszalin (Köslin) [WA70]. VI 1912. 1 ex.. leg. ZM [USMB].

A halophilous species, living in seacoasts in western Europe, southern Scandinavia, southern coasts of the North and Baltic Sea. Specimens deposited in the reviewed collections have been captured in the sea coast from Wolin to Vistula Spit, i.e., in whole Polish part of the Baltic Coast. The occurrence of the species in Pomeranian Lake District has not been recorded in KFP, but it seems likely in view of its general distribution in Poland.

\section{Pedinus femoralis (Linnaeus, 1767)}

Mazowiecka Lowland: Jabloń [EC17], 8 VI 1896, 1 ex.. leg. WM [USMB]; WarszawaAnin [EC18], 1949. 1 ex.. leg. ZA [MIZ].

Malopolska Upland: Klonów [DA47] ad Miechów, 23 IX 1965, 1 ex.. leg. WS [ISEA]; Dale [DA47] ad Raclawice. 8 VI 1965 and 13 X 1967. 4 exx.. leg. WS [ISEA]: Morsko [DA65] ad Nowy Korczyn, 5 XI 1996, 1 ex.. leg. TW [ISEA]; Krzyżanowice [DA68], 17-19 VIII 1952. 
2 exx., leg. „staff of PMZ”, 8-13 V 1953, 2 exx., leg. RB, 9 VIII 1954, 1 ex., leg. ME, 25 VIII 1954. 1 ex.. leg. EN [MIZ], 6 VIII 1953 - 8 V 1979. 5 exx.. leg. WS \& MM [ISEA]: Winiary Zagojskie [DA69]. 9 IX 1954, 1 ex., leg. BB [MIZ]: Skowronno [DA69]. 28 VII 1964, 1 ex.. leg. WS [ISEA]. 10 VII 1994. 18 VI 1995, 15 VII 1996. 3 exx.. leg. RD\&WZ [USMB]: Pińczów [DA69], 4 VII 1950, 2 exx., leg. RB, 30 VIII-30 IX 1950, 10 exx., leg. BB, 29 VIII 1950 and 7 IV 1953, 5 exx., leg. JF [MIZ], 11 VII 1996, 1 ex., leg. TW [ISEA]: Skorocice [DA78], 5-8 V 1979. 4 exx.. leg. MM [ISEA]: Bogucice [DA79]. 26 V 1953. 1 ex.. leg. WS [ISEA]. 5 VI 1951. 1 ex., leg. GO, 15 V 1954, 1 ex., leg. BB [MIZ]: Welecz [DA79]. 25 VI-8 VII 1951, 18 V 1952. 4 exx.. leg. JF [MIZ]: Galów [DA79]. 26 V 1993, 2 exx.. leg. MM [ISEA]: Szaniec [DA79]. 29 IV 1993, 1 ex.. leg. MM [ISEA]: Chotel Czerwony [DA88], 20 V 1952, 1 ex., leg. BB [MIZ], 18 IX 1963 and 8 V 1967. 2 exx.. leg. WS, 8 V 1980. 1 ex., leg. WW [ISEA]: Busko-Zdrój [DA89]. 30 VIII-3 IX 1950, 8 exx., leg. BB, 3 VII 1950, 1 ex., leg. RB, 8 IX 1950 and 24 VI-11 VII 1951. 5 exx.. leg. JF [MIZ]: Kików [DA98]. 23-28 V 1952. 3 exx.. leg. BB [MIZ]. 6 VI 1991. 2 exx.. leg. JM [ISEA], 19 VI 1995, 1 ex., leg. RD [USMB].

Lubelska Upland: Kazimierz Dolny [EB68]. V 1934, 1 ex., leg. TE. 30 V-1 VI 1948, 2 exx.. leg. RB, 14-15 V 1949, 2 exx., leg. GO \& AR, 11 VII 1950 and 21 V 1956, 2 exx., leg. RB [MIZ]. 14 V 1963. 1 ex.. leg. WS [ISEA]; Tarnogóra [FB44] ad Krasnystaw. 2 VII 1985. 1 ex.. leg. JM [ISEA]: Gródek [GB03] ad Hrubieszów, 10 VII 1962 - 5 V 1965. 25 exx.. leg. WS [ISEA].

Roztocze Upland: Zwierzyniec [FB40], 12 VIII 1987, 1 ex.. leg. JG [USMB]; Dziewcza Góra [FB41] ad Niedzieliska. 9 VI 1989, 1 ex., leg. JP [ISEA].

Sandomierska Lowland: Tarnogóra [EA97]. 26 VII 1987, 2 exx., leg. DI [USMB].

Eastern Beskidy Mts.: Łuczyce [FA31] ad Przemyś1, 18 IV 1880, 18 exx., leg. BK [ISEA].

A xerothermophilous species, occurring in south-eastern Poland. Faunistic data obtained from the reviewed collections confirm its occurrence in Malopolska Upland (voucher specimens for notes of B. Burakowski* are deposited in MIZ collection) and in Lubelska Upland. Undoubtedly, it inhabits both Roztocze Upland and Sandomierska Lowland (not listed in KFP, the specimens are kept in ISEA and USMB collections), while its occurrence in Mazowiecka Lowland is not certain (a single specimen collected by W. MĄcZYŃSKi in USMB collection; the finding can be explained by crossing the river or introduction).

\section{Opatrini Brullé, 1832}

\section{Gonocephalum granulatum pusillum (FABRICIUS, 1792)}

Mazowiecka Lowland: Jabłoń [EC17], 8 V 1896, 1 ex.. leg. WM [USMB].

In 1993, FERRER proposed the status of subspecies for this taxon, i.e. G. gramulatum pusillum (FABR.), which was further confirmed and formally accepted (FERRER 1995; IwAN \& LổL 2008). In KFP, the species was not included into Polish fauna (no index number was given). It was reported from Lower Silesia more than 160 years ago (UECHTRITz 1848), but further excluded by GERHARDT (1910). The specimen from 
W. MAczYÝski`s collection (USMB) does not support this report, but merely raises a question whether a single beetle collected more than 110 years ago can prove the occurrence of this species in Poland in the past.

\section{Opatrum riparium $\mathrm{SCRIBA}, 1865$}

Baltic Coast: Rowy [XA35] ad Ustka, 21 VII 1970. 3 exx.., leg. JP [ISEA]; Gdańsk-Rokitnica (Müggenhahl Danzig) [CF41]. V-VI 1919. 8 exx.. leg. KN [USMB].

Wielkopolska-Kujawy Lowland: Glogów (Glogau) [WT72], V 1935, 1 ex., coll. USMB.

Mazowiecka Lowland: Warszawa-Natolin [EC08], 11 V 1899, 1 ex., leg. WM [USMB].

Upper Silesia: Łężczok (Lenczok Ratibor) [CA05]. 31 X 1937. 1 ex.. leg. HN [USMB]; Nędza [CA06], 18 IX 1920, 4 exx., coll. USMB; Ciechowice [CA06] ad Nędza, 5 V 1918. 2 exx.. coll. USMB: Gliwice-Łabędy (Laband) [CA38]. 24 VI 1925. 1 ex., leg. KI. 27 III 1928. 21 IV 1932. 7 V 1938. 4 exx.. leg. HN [USMB]: Oświęcim [CA74]. 26 IV 1930. 1 ex.. leg. ZT [USMB]: Dąbrowa Górnicza (Dobrau) [CA77], 8 XI 1936 and 21 V 1938, 3 exx.. coll. USMB: Ciężkowice [CA86] ad Jaworzno. 5 IV 1920, 1 ex.. leg. SS [ISEA].

Kraków-Wieluń Upland: Kraków-Dębniki [DA14], ca. 1900, 1 ex.. leg. MR [ISEA]; Kraków-Pychowice [DA14]. 6 X 1919. 1 ex., leg. SS [ISEA]; Kraków-Wola Justowska [DA14]. 23 VI 1911 and 21 V 1918. 3 exx.. leg. SS \& SP [ISEA]: Kraków-Przegorzały [DA14]. 30 IV 1915. 1 ex.. leg. SS [ISEA]: Kraków-Plaszów [DA24], 25 IV 1935. 7 exx.. leg. SP [ISEA]

Malopolska Upland: Malkowice [DA76] ad Koszyce. 14 V 1991, 1 ex., leg. MM [ISEA].

Sandomierska Lowland: Grodkowice [DA43] ad Klaj. 1 V 1925 and 13 IV 1926. 10 exx.. leg. SS [ISEA]: Zaleszany [EB61] ad Sandomierz. 1-10 IX 1890, 14 IV-13 V 1891, 31 exx., leg. SS [ISEA].

Western Beskidy Mts.: Zator [CA83]. 3 V 1953, 1 ex.. leg. JS [ISEA]; Kraków-Kosocice [DA23], brackish place, $12 \mathrm{~V} 1994,1$ ex.. leg. DK [ISEA].

Eastern Beskidy Mts.: Łuczyce [FA31] ad Przemyśl, 1878, 1 ex., leg. BK [ISEA]; Hurko [FA31] ad Przemyśl. 3 V 1883 and 3 V 1884. 3 exx.. leg. BK [ISEA].

A widespread species in the southern part of northern Europe and in central Europe (Burakowski et al. 1987). It is rarely met in Poland in few scattered localities. The specimens deposited in ISEA have been captured in Wyżyna Malopolska (Malopolska Upland) and in Eastern and Western Beskid Mountains (not listed in KFP). In MIZ collection, there are no voucher specimens for notes signed "B. BURAKOWSKI*".

\section{Opatrum sabulosum sabulosum (LinNaEus, 1761)}

Baltic Coast: Gdańsk (Danzig) [CF42], date missing. 2 exx., coll. USMB; Sopot [CF43]. 8-9 V 1950, 2 exx., leg. KR [MIZ]: Gdynia [CF44], 2 VIII 1931, 1 ex., leg. SS [ISEA].

Pomeranian Lake District: Bielinek nad Odra [VU46]. 30 V 1983, 1 ex.. leg. DI [USMB]; Ślesin [XU79], 21 V 1995, 1 ex.. leg. DK [ISEA]: Grabowo [CE10]. Kozielec nat. res.. 23 V 1995, 30 V 1996, 2 exx., leg. DK [ISEA]: Gruczno [CE21], 22 V 1995, 30 V 1996, 2 exx.. leg. DK [ISEA]: Phutowo [CE21]. 24 VI 1964. 4 VI 1965, 3 exx.. leg. WS [ISEA]: Gniew [CE56], 18 VI 2001. 1 ex.. leg. DK [ISEA]: Tczew-Górki [CE59]. 17 V 2003. 1 ex.. leg. DK [ISEA]: Biala Góra [CE67] ad Sztum, 16 V 2003, 1 ex.. leg. DK [ISEA]. 
Masurian Lake District: Świecie [DE00] ad Brodnica, 15 V 2003, 1 ex., leg. DK [ISEA]; Elk [EE86], 20 III 1948, 1 ex., leg. RB [MIZ]: Szyba [EE86] ad Ełk, 20 III 1948, 1 ex.. leg. RB [MIZ]: Grajewo [EE94]. 27 X 1947 and 27 II 1948, 2 exx.. leg. RB [MIZ].

Wielkopolska-Kujawy Lowland: Zielona Góra [WT35]. 21 X 1950, 1 ex., leg. RB [MIZ]; Rybojady [WU50]. 15 VII 1961. 1 ex., coll. USMB; Biała [XU08] ad Trzcianka, 18 V 2002. 1 ex.. leg. MM [ISEA]: Luboń [XU20]. 7 V 1975. 1 ex.. leg. PS [USMB]: Suchy Las [XU21]. 11 V 1974, 1 ex., leg. LB [USMB]; Kobylnica [XU41] ad Swarzędz, 27 V 1951, 1 ex., leg. RB [MIZ]: Wierzonka [XU41] ad Swarzędz, 10-15 VI 1947, 2 exx., leg. WE [MIZ]: Bałdoń [CC30] ad Kalisz, 8 V 1988, 1 ex.. leg. AM [ISEA]: Kolo [CC38]. 6 VII 1958. 1 ex.. leg. BB [MIZ]: Borek [CD18] ad Toruń, 18 VIII 1964, 4 exx.. leg. WS [ISEA]: Miszek [CD55]. 16 VI 1976. 1 ex., leg. JG [USMB]; Ciechocinek [CD56], 26 V 1949, 1 ex., leg. RB [MIZ]; Wlocławek: Kulin nat. res. [CD63]. 3 V 1951, 2 exx.. leg. RB [MIZ]: Bobrowniki [CD65]. 14 V 2001, 1 ex.. leg. DK [ISEA].

Mazowiecka Lowland: Rawka [DC45] ad Skierniewice, 22 VII 1957, 4 exx., leg. BB [MIZ]: Grodzisk Mazowiecki [DC77]. 26 VII 1964 and 4 VII 1965, 5 exx., leg. BB [MIZ]: Zaborów [DC79]. 9 IX 1956, 2 exx., leg. BB [MIZ]; Grabina [DC79] ad Warszawa, 7-8 V 1950, 2 exx., leg. BB [MIZ]; Puszcza Kampinoska Forest: Sieraków [DC89], 15 IV 1953. 2 exx., leg. BB [MIZ]; Puszcza Kampinoska Forest: Wydma Łuże [DC89]. 5 VI 1965. 3 exx.. coll. MIZ; Kampinoski N. P., Lipków [DC89], 8 V 2003, 2 exx.. leg. WJ [ISEA]; Dziekanów Leśny [DC99], 1-27 IX 1957 and 5 IV 1959, 4 exx., leg. BB [MIZ]: Warszawa-Bielany [DC99]. 2 V 1890, 2 exx.. leg. WM [USMB]: Warszawa-Natolin [EC07]. 14 VI 1893, 2 exx., leg. WM [USMB]; Warszawa-Saska Kępa [EC08], 26 V 1928, 2 exx.. leg. HC [MIZ]; Pustelnik [EC09]. 5 VI 1955, 28 IX 1958, 26 VIII 1964, 4 exx., leg. BB [MIZ]; Warka [EC13]. 5 IV 1949, 2 exx., leg. MZ [MIZ]: Obory [EC16], 19 VIII 1950, l ex., leg. BB [MIZ]; Jabłoń [EC17], 1 VII 1901. 1 ex., leg. WM [USMB]: Świder [EC17], 4 VII 1903, 1 ex.. leg. WM [USMB]: Stara Miłosna [EC18], 19 VI 1958, 1 ex., leg. BB [MIZ]; Sewerynów [EC21], 30 VII 1961, 1 ex., leg. BB [MIZ]; Celestynów [EC26] ad Warszawa, 3 VII 1932, 1 ex., leg. SS [ISEA]: Struga [ED00]. 1 VI 1892, 1 ex.. leg. WM [USMB]. 2 X 1958, 1 ex., leg. BB [MIZ]: Klembów [ED20]. 21 X 1956, 1 ex., leg. BB [MIZ]; Urle [ED41] ad Wyszków, 10 VI 1909. 1 ex., leg. WM [USMB]: „Warszawa”, 21 V 1891, 1 ex., leg. WM [USMB].

Podlasie Lowland: Kryńszczak [EC95] ad Łuków. 16 V 1953, 1 ex.. leg. WB [MIZ]; Majdan Stuleński [FB89] ad Wlodawa, 17 VI 2003, 1 ex.. leg. MM [ISEA]; Niemirów [FC49], 12 V 2003, 1 ex., leg. DK [ISEA]: Mielnik [FD40], 6 VI 1963, 5 IX 1969, 4 exx., leg. WS \& JP [ISEA]; Pilatowszczyzna [FD79]. 3 V 1992. 3 exx.. leg. JP [ISEA].

Bialowieża Primeval Forest: f. comp. 47D [FD85]. 17 IV 1973, 1 ex., leg. JL [USMB]; Białowieża [FD94], 30 IV 1977. 2 exx.. leg. MB [ISEA]: Siemianówka [FD96]. 18 IV 1916. 1 ex., leg. JO [USMB].

Trzebnickie Hills: Trzebnica (Trebnitz) [XS49], VI 1935, 5 exx., leg. KI [USMB].

Upper Silesia: Gogolin [BA89], 30 IV 1928, 1 ex., leg. HN. 5 III 1929, 5 V 1929, 7 IV 1936. 18 exx.. leg. KI [USMB]: Pogrzebień [CA05] ad Racibórz, 10 VIII 1932, 1 ex.. leg. WY [USMB]: Rybnik-Paruszowiec (Paruschowitz) [CA25], 17 IV 1910, 1 ex., leg. SC [USMB]; GliwiceBrzezinka (Birkenau) [CA37], 12 VI 1936, 1 ex., leg. KT [USMB]; Brynek [CA39], 26 VI 1925. 1 ex.. coll. USMB; Mikołów-Mokre (Mokrau) [CA46]. 16 V 1932. 2 exx.. coll. USMB; Czechowice-Dziedzice [CA53]. VI 1925, 1 ex.. leg. WM [USMB]; Mikolów-Jamna [CA55]. 9 VI 1938. 1 ex., leg. KW [USMB]; Ruda Śląska-Kochłowice [CA57], 5 VI 1938, 4 VII 1939, 21 VI 1965. 3 exx.. leg. KW [USMB]: Bytom (Beuthen) [CA58]. 10 VI 1932. 8 exx.. leg. KI [USMB]: Chelmek [CA75], 5 V-30 IX 1878, 1 VII 1880, 10 IV 1881, 19-28 IV 1882, 21 III 1883, 12 IX 1884. 
5 IV 1885, 15 V 1898, 3 IX 1921, 29 V 1931, 12 VI 1932, 27 exx., leg. SS [ISEA], 28 IV 1932. 2 exx., leg. WM [USMB]; Libiąż [CA75] ad Chrzanów, 7 IX 1879, 2 VII 1890, 16 V 1932, 3 exx.. leg. SS [ISEA]; Jaworzno-Szczakowa [CA76], 12 VII 1910, 2 exx.. leg. SS [ISEA]; Dhugoszyn [CA76] ad Jaworzno-Szczakowa, 30 VI 1910, 2 exx., leg. SS [ISEA]; Dąbrowa Górnicza [CA77]. VII 1936. $21 \mathrm{~V}$ and 5-15 IX 1938, 4 exx., coll. USMB; Żarki [CA84] ad Chrzanów, 20 V-15 VI 1881. 2 exx.. leg. SS [ISEA]: Pogorzyce [CA85] ad Libiaż. 2 VII 1880, 1 ex.. leg. SS [ISEA]: Szymiszów (Schemischow) [CB00], 16 V 1928, 1 ex., leg. KI [USMB]; Byczyna [CB06]. VII 1955, 1 ex., leg. SI [USMB]; Zawadzkie [CB20]. 2 XI 1930, 1 ex.. leg. HN [USMB].

Kraków-Wieluń Upland: Ogrodzieniec [CA98]. 23 IV 1952. 2 exx.. leg. MZ [MIZ]; Karlin [CA99] ad Zawiercie. 24 IV 1952. 1 ex., leg. MZ [MIZ]: Cieciulów [CB35]. 29 V 1932 and 20 V 1937, 5 exx., coll. USMB; Góra Zborów (Berkowa Góra) [CB90]. 24 IV 1952, 3 exx., leg. MZ [MIZ]; Przeginia Duchowna [DA04] ad Czernichów. 29-31 V 1906. 15 VI 1931. 4 exx.. leg. SS, 21 V 1976, 1 ex.. leg. MG [ISEA]; Krzeszowice [DA05], 10 X 1916. 1 ex.. leg. SS [ISEA]: Rudawa [DA05], 20 X 1917, 20 XI-10 XII 1931, 3 exx.. leg. SS [ISEA]; Ściejowice [DA13] ad Liszki, 27 X 1898, 8 exx.. leg. SS [ISEA]; Kraków-Wola Justowska [DA14]. 15 IV-6 IX 1878, 25 IX 1892, 17 VI 1917, 4 VI 1931, 5 exx., leg. SS [ISEA]; Kraków-Sikornik [DA14], 11 V 1911, 19 VI 1917, 11 IV 1939, 6 exx., leg. SP [ISEA]; Kraków-Przegorzały [DA14], 30 IV 1915. 7 V-22 X 1917. 2 V 1920, 12 exx.. leg. SS [ISEA]: Kraków-Bielany [DA14]. 29 V 1919. 19 VI 1932, 1 V-19 VII 1967, 10 V 1972, 2 IV 1989, 6 exx., leg. SS. MG \& JP [ISEA]; Kraków: Lasotnia [DA14], ca. 1900, 2 exx., leg. MR [ISEA]; Kraków-Krzemionki [DA14], 24 V 1878. 12 V 1901. 28 IV 1906, 17 VI 1916. 25 IX 1921. 14 VI 1925. 21 VIII 1932. 18 IV 1935. 17-19 IX 1936, 27 VIII 1938, 27 IV 1942, 23 exx., leg. SS, SP \& R. Wojtusiak [ISEA]; Kraków-Pychowice [DA14], 5 V 1936, 2 exx.. leg. SS [ISEA]; Kraków-Podgórki [DA14], 15 IV 1961, 22 VI 1965, 2 V 1966, 15 IX 1967, 6 IV 1971, 6 exx.. leg. WS \& ZS [ISEA]: Kraków-Tyniec [DA14]. 15 III-1 IV 1898, 4 exx., leg. SS [ISEA]; 14 IV 1984, 3 IV 1985, 10 VI 1987. 21 III 1989. 8 exx.. leg. DK, JP \& JM [ISEA]; Modlniczka [DA15] ad Kraków, 16 VIII 1900, 3 exx.. leg. SS [ISEA]; Jerzmanowice [DA16] ad Ojców. 1 VI 1966, 1 ex.. leg. AS [ISEA]; Ojców [DA16], 23 VII 1961. 1 ex.. leg. PK [MIZ]: Ojców-Grodzisko [DA16]. 25 V 1964. 22 X 1968. 2 exx.. leg. WS [ISEA]: Kraków: Wawel [DA24], 20 IV 1971, 2 exx., leg. MG [ISEA]; Kraków-Olsza [DA24]. 1 V 1918. 1 ex.. leg. SP [ISEA]; Kraków-Łobzów [DA24]. 29 VI 1933, 3 exx.. leg. SS [ISEA]; KrakówPrądnik Bialy [DA24], 11 V 1990, 1 ex., leg. MM [ISEA]: Kraków-Mydlniki [DA24], 10 VI 1906, 1 ex., leg. SS [ISEA]; Mirów [CB90], 9 V 2003, 1 ex., leg. WJ [ISEA].

Malopolska Upland: Łódź-Łagiewniki [CC94], $19 \mathrm{~V}$ 1969, 1 ex., leg. JC [USMB]; Uniejów-Rędziny [DA28] ad Miechów, 2 V 1965, 18 V 1966, 2 exx.. leg. WS [ISEA]; Lisiniec [DA37] ad Miechów, 16 VI 1953, 1 ex., leg. WS [ISEA]; Dale [DA47] ad Raclawice, 6 V 1961. 10 VII 1964, 2 V 1966, 13 X 1967, 5 exx.. leg. WS [ISEA]; Pelczyska [DA68] ad Wiślica, 3 XI 1992, 2 exx.. leg. JP [ISEA]; Krzyżanowice [DA68], 28 V 1950. 1 ex.. leg. WB. 9 VIII 1953. 2 exx., coll. MIZ, 1 V 1972, 8 V 1979, 4 exx., leg. WS \& MM [ISEA]; Mozgawa [DA68] ad Pińczów, 3 IV 1990, 1 ex., leg. JP [ISEA]; Winiary Zagojskie [DA69], 9 IX 1954, 1 ex., leg. BB [MIZ]: Pińczów [DA69], 4 VII 1950, 1 ex., leg. RB, 29 VIII 1950, 1 ex., leg. JF. 30 IX 1950. 2 exx., leg. BB [MIZ]. 6 VI 1991. 2 exx., leg. MM [ISEA]; Malkowice [DA76] ad Koszyce. 31 V 1991. 1 ex., leg. MM [ISEA]: Szczerbaków [DA77] ad Busko Zdrój. 22 V 1952 and 21 V 1953. 2 exx.. leg. BB [MIZ]: Skorocice [DA78]. 10 IX 1963, 1 ex.. leg. BB [MIZ]. 16 VI 1965. 5 V-10 XI 1979. 3 exx., leg. WS \& MM [ISEA]: Welecz [DA79]. 18 V 1952, 2 exx.. leg. JF [MIZ]; Bogucice [DA79], 22 VI 1962, 30 IX 1967, 3 exx.. leg. WS [ISEA]; rez. Grabowiec [DA79]. 12 VII 1996. 1 ex.. leg. RD [USMB]: Pasturka [DA79] ad Pińczów. 19 VI 1997. 1 ex., leg. WZ [USMB]: Żerniki Górne [DA88]. 6 VI 1991 and 18 V 1992, 2 exx., leg. MM 
\& JP [ISEA]; Chotel Czerwony [DA88]. 19 VI 1953, 8 V-16 XI 1979, 5 exx., leg. WS \& MM [ISEA]: Busko-Zdrój [DA89], 24-30 VI 1951, 2 exx., leg. JF [MIZ]: Kików [DA98], 23 V 1952. 1 ex., leg. BB [MIZ]. 6 VI 1991. 1 ex.. leg. JM [ISEA]. 19 VI 1995. 1 ex., leg. WZ [USMB]: Skowronno [DB60], 3 IV 1990, 3 VI 1991, 3 exx., leg. JP [ISEA]; Radom [EB19]. 24 VI 1897. 4 exx., leg. SS [ISEA]; Gorzyczany [EB40] ad Sandomierz, 26 VII 1971, 1 ex.. leg. WS [ISEA]; Sandomierz [EB51]. 13 IV 1949. 3 exx.. leg. RB \& MZ [MIZ]: Góry Pieprzowe [EB51]. 5 V 1951, 18 IV-18 IX 1952, 22 III-26 X 1953, 13 V-22 VII 1954, 5 V 1972, 17 exx., leg. WS [ISEA]: Dwikozy [EB52] ad Sandomierz, 31 V 1967, 2 exx., leg. WS [ISEA]; Maruszów [EB53] ad Annopol. 2 VI 1985. 1 ex.. leg. JP [ISEA].

Świętokrzyskie Mts.: Zelejowa Góra [DB53] ad Chęciny, 6 VI 1958, 26 V 1963, 18 VII 1964, 3 IX 1965, 2 V-21 VI 1967, 10 exx., leg. WS [ISEA]; Nowa Shupia [EB03] ad Kielce, 24 VI 1966. 1 ex.. leg. WS [ISEA].

Lubelska Upland: Pulawy [EB69]. 15 X 1983, 1 ex.. leg. DI [USMB]; Tarnogóra [FB44] ad Krasnystaw, 7 IX 1972, 1 ex., leg. WS [ISEA]; Labunie [FB61] ad Zamość, 8 VI 1970, 1 ex., leg. ZS \& MO [ISEA]: Stawska Góra [FB67] ad Chelm. 10 V-7 VI 1963. 17 IX 1965. 5 exx.. leg. WS [ISEA]; Gródek [GB03] ad Hrubieszów, 10 VII 1962, 9 V-7 VI 1963, 5 V 1965, 10 VI 1970, 4-7 VI 1971, 24 V 1987, 18 exx., leg. WS, ZS, MO \& MM [ISEA]: Ślipcze [KS82] ad Hrubieszów. 1 V 1964, 1 ex.. leg. TP [MIZ].

Roztocze Upland: Zwierzyniec-Sochy [FB30], 13 V 1987, 2 exx., leg. MM [ISEA]; Zwierzyniec [FB30]. 12 VIII 1987. 1 ex., leg. JG [USMB]; Zwierzyniec [FB30], Bukowa Góra. 12 V 1987. 3 exx.. leg. LB [USMB].

Sandomierska Lowland: Węgrzce Wielkie [DA34] ad Kraków. 22 VIII 1932, 1 ex.. leg. SS [ISEA]: Kłaj [DA53], 1904, 16 exx., leg. MR, 13 VI 1911, 15 VII 1934, 23 VI 1935, 4 exx., leg. SS [ISEA]: Tarnów [DA94], 22 VII 1941. 1 ex., leg. SS [ISEA]: Dąbrówki Breńskie [DA96]. 2-4 VII 1942, 1 ex., leg. SS [ISEA]: Rzeszów [EA74], 27 VII 1917, 1 ex., leg. SS [ISEA]: Zbydniów [EB60] ad Rozwadów, 2 V 1891, 1 ex., leg. SS [ISEA]; Stalowa Wola [EB70], 13 IV 1952, 3 exx., leg. WE [MIZ]; Pawłosiów [FA13] ad Jarosław, 2 VI 1889, 1 ex.. leg. SS [ISEA]: Jaroslaw [FA24]. 28 IV 1889, 1 ex., leg. SS [ISEA]: Horyniec [FA66]. 1-30 VI 1888, 1 ex.. leg. SS [ISEA].

Western Beskidy Mts.: Polanka Wielka [CA73] ad Oświęcim. 21 IV 1899, 1 ex.. leg. SS [ISEA]: Skawina [DA13]. 2-16 VI 1932, 9 exx., leg. SS [ISEA]; Kraków-Borek Falecki [DA23]. 6 VI-7 X 1931, 4 VI 1932, 18 exx., leg. SS [ISEA]: Tęgoborza [DA70], 25 IV-3 VI 1892, 6 exx., leg. SS [ISEA]; Chełmiec [DV79], 12 IV-25 V 1892. 2 exx., leg. SS [ISEA]; Nowy Sącz [DV79]. 31 III-30 V 1892. 3 exx.. leg. SS [ISEA]: Żegiestów [DV87]. 26 IV-16 VI 1892. 4 exx.. leg. SS [ISEA].

Eastern Beskidy Mts.: Wapowce [FA11]. $10 \mathrm{~V}$ 1884, 1 ex., leg. BK [ISEA]; Hohubla [FA11]. 31 V 1884, 1 ex.. leg. BK [ISEA]; Makowa [FA20]. 8 V 1990. 1 ex.. leg. JM [ISEA]: Przemyśl: Trzy Krzyże [FA21], 29 IV 1878, 10-19 IV 1883, 5 exx.. leg. BK [ISEA]; Przemyśl-Winna Góra [FA21],30 III-14 IV 1880, 11 XI 1882 and 3 IV 1883, 9 exx.. leg. BK [ISEA]: Przemyśl-Wzniesienie [FA21], 18-19 IV 1882, X-XI 1882, 29 III 1884, 4 exx.. leg. BK [ISEA]: Przemyśl-Zasanie [FA21]. 12 IV 1883, 1 ex., leg. BK [ISEA]; Przemyśl: Krzyż Zielonki [FA21]. 18 III-28 IV 1883, 27 IX 1886, 5 exx.. leg. BK [ISEA]: Hurko [FA31], 29 IV-3 V 1883, 4 exx.. leg. BK [ISEA]: Luczyce [FA31]. 1877-1878. 18 IV 1880. 6 exx.. leg. BK. 24 VI 1965. 1 ex.. leg. WS [ISEA].

Pieniny Mts.: Czorsztyn [DV47]. Góra Zamkowa, 19 VI 1967, 1 ex.. leg. WS [ISEA]; Pulsztyn Mt. [DV57], 19 VII 1959, 1 ex., leg. WS [ISEA]; Cisowiec Mt. [DV57], 6 VI 1964, 1 ex.. leg. WS [ISEA].

Tatry Mts.: Polana Rusinowa [DV35], 6 VIII 1881, 2 exx., leg. BK [ISEA]. 
The Opatrum sabulosum species consists of seven subspecies, six of which are endemic for Spain, southern Europe and Caucasus, while one, O. sabulosum sabulosum, is widespread everywhere in Palaearctic, including Poland. It is one of the most frequently captured darkling beetles in our country. It occurs in all its territory, though it has not been reported from some regions in KFP. Very numerous in the studied collections. Basing on the specimens kept in ISEA, Trzebnickie Hills, Świętokrzyskie Mountains and Pieniny Mts. can be formally included to KFP regions where occurrence of this species has been documented in publications. In the MIZ collection, there are voucher specimens for records signed B. BURAKOWSKI*.

\section{Platyscelidini LACORDAIRE, 1859}

\section{Oodescelis melas (Fischer von WALDHEIM, 1823)}

Mazowiecka Lowland: Czersk [EC15]. 21 IV 1918, 1 ex.. leg. BL [MIZ].

Podlasie Lowland: Radomyśl [EC96], 12 VII 1959, 1 ex., leg. CS. 19 VII 1959. 1 ex., leg. PARDUS [ISEA] (determined as Oodescelis polita!).

Malopolska Upland: Galęzice [DB53]. 3 VI 1916. 1 ex.. leg. TE [MIZ].

Lubelska Upland: Kazimierz Dolny [EB68], 3 VI 1914, 1 ex., leg. TE [MIZ]; Pulawy [EB69]. 24 IV 1925, 1 ex., leg. HC [MIZ].

The species reaches Poland from south-eastern Europe. Reported in KFP from three south-eastern regions. CIEŚLAK (2004) confirmed its occurrence in Sandomierska Lowland and Lubelska Upland. Specimens deposited in the reviewed collections come from single localities in Malopolska Upland, Lubelska Upland and Mazowiecka Lowland, which has not been reported previously. The species is listed in Polish Red List, EX? category (PAwLOwski et al. 2002), which requires verification in light of the abovementioned publication. In ISEA collection, there is also a misidentified specimens from the Podlasie Lowland (vicinity of Siedlce), on the basis of which Oodescelis polita (STuRm, 1807) was reported from Poland by Szymczakowski (1973), which was later repeated in KFP.

\section{Melanimini Seiduitz, 1894}

\section{Melanimon tibialis tibialis (FABRICIUS, 1781)}

Baltic Coast: Pobierowo [VV99] ad Szczecin, 10 VI 1980, 1 ex.. leg. CW [ISEA]; Jastrzębia Góra [CF27], 27 VIII 1936, 1 ex., leg. AB [MIZ], 24 VI 1996. 1 ex., leg. RD [USMB]; Karwia [CF27]. 10 VIII 1963, 1 ex., leg. RB [MIZ], 15 VI 1996, 16 exx.. leg. RD [USMB]: Jastarnia [CF36], $30 \mathrm{~V}$ 1978, 1 ex., leg. JG [USMB]: Wladyslawowo [CF37], 6 VI 1993, 4 exx., leg. RD [USMB]; Gdańsk (Danzig) [CF42], date missing, 2 exx., coll. USMB; Gdańsk-Brzeźno [CF43]. 7 VI 1948, 3 exx., leg. RB [MIZ]: Sopot [CF43]. 2 VI 1987. 1 ex.. leg. RK [ISEA]; Jurata 
[CF56]. 2-12 VI 1972, 3 exx., leg. ZS [ISEA]; Elbląg [CF90]. 21 V 1986, 1 ex.. leg. PZ [ISEA]; Mierzeja Wiślana (Frische Nehrung), date missing, 3 exx., leg. KN [MIZ]; Zalew Wiślany (Frisches Haff), date missing, 2 exx.. coll. [USMB].

Pomeranian Lake District: Bielinek nad Odrą [VU46]. 3 V 1984, 1 ex.., leg. PS [USMB]; Szczecin (Stettin) [VV62], date missing, 11 exx., leg. FK [MIZ]; Goleniów (Gollnow) [VV83]. date missing. 2 exx.. leg. LD [MIZ]; Szczecinek [Neustettin] [XV15]. VI 1940, 1 ex.. leg. HN [ISEA]; Koszalin (Coeslin) [WA70], date missing. 8 exx., leg. AL [MIZ]; Grabowo [CE10]. Kozielec nat. res., $23 \mathrm{~V}$ 1995, 1 ex., leg. MM [ISEA]; Wzgórza Phutowskie Hills [CE20]. 4 VI 1965. 9 exx.. leg. WS. 24 V 1995. 27 V 1996. 2 exx.. leg. MM \& DK [ISEA]: Chrystkowo [CE21] ad Gruczno, 30-31 V 1996, 2 exx., leg. DK [ISEA]: Gruczno [CE21]. 23 V 1995. 31 V 1996, 2 exx., leg. MM \& DK [ISEA]; Terespol Pomorski [CE22], 1 VI 1990, 1 ex., leg. JP [ISEA]: Jamy nat. res. [CE54] ad Gardeja. 15 VII 1926. 1 ex.. leg. TO [USMB].

Wielkopolska-Kujawy Lowland: Nowogród Bobrzański [WT13]. 9 V 1982 and 18 V 1984. 4 exx., leg. PS [ISEA, USMB]; Bocianowo [BD99] ad Bydgoszcz, 31 V 1927, 1 ex., leg. TO [USMB]: Wielkopolski N. P.. f. comp. 4 [XT29]. 28 VIII 1986. 1 ex.. leg. PS [ISEA]: Rogalin [XT39], 5 V 1984, 1 ex.. leg. DI [USMB]; Brzostków [XT67]. 1 V 1991, 1 ex., leg. AM [ISEA]; Poznań-Golęcin [XU21], 5 VI 1930, 1 ex., leg. HC [MIZ]: Poznań-Dębina [XU30]. 19 VIII 1985. 18 exx.. leg. MA [ISEA]: Krzesiny [XU30] ad Poznań. 21 V 1986, 1 ex.. leg. MA [ISEA]: Potasze [XU32] ad Poznań. 1 VII 1984, 15 exx., leg. JW [USMB]; Zielonka [XU42] ad Poznań. 26 VI 1974, 1 ex., leg. PS [USMB]; Bałdoń [CC30] ad Kalisz, 6 IV 1985, 7 III 1989, 2 exx.. leg. AM [ISEA]: Łęczyca [CC76]. 13 III 1938, 1 ex., leg. AB [MIZ]: Borek [CD 18] ad Toruń. 25 VI 1964, 1 ex., leg. WS [ISEA]; Wielka Kępa Ostromecka nat. res. [CD19] ad Bydgoszcz. 29 V 1996, 1 ex.. leg. DK [ISEA]: Kulin nat. res. [CD63] ad Wloclawek, 3 V 1951, 1 ex.. leg. RB [MIZ].

Mazowiecka Lowland: Otwock [DC46]. VIII 1923, 2 exx., leg. EM [MIZ]; Julinek [DC79] ad Leszno, 26 X 1958, 2 exx., leg. BB [MIZ]; Zaborów [DC79], 9 IX 1956, 2 exx., leg. BB [MIZ]; Rozalin [DC86]. 14 VI 1955, 2 exx.. leg. MZ [MIZ]; Puszcza Kampinoska Forest. Wydma Łuże [DC89]. 5 VI 1965, 9 exx.. coll. MIZ: Kampinoski N. P.. Lipków [DC89]. 26 V 2003. 1 ex., leg. WJ [ISEA]; Phudy [DC99]. 14 IX 1947, 1 ex., leg. RB [MIZ]: Warszawa-Koło [DC99]. 9 V 1948, 4 exx.., leg. RB [MIZ]: Warszawa-Bielany [DC99]. 2 V 1890. 1-12 VI 1897. 3 VI 1898 and 27 VI 1917. 6 exx., leg. WM [USMB], 3 VI 1950, 2 exx.. leg. RB [MIZ]: Grochale [DD70] ad Kazuń, 12 V 2001, 1 ex., leg. DK [ISEA]; Pomiechówek [DD81]. 10 III and 1 IV 1928. 2 exx.. leg. AB, 21 V 1948, 1 ex., leg. MZ, 21 V 1949, 1 ex., leg. RB [MIZ]; Jabłonna [DD90]. 4 VI 1893, 1 ex.. leg. WM [USMB]: Chojnów [EC06]. 4 VI 1932, 1 ex.. leg. TE, 16 V 1948. 1 ex., leg. ZW [MIZ]; Skolimów [EC07]. 23 V 1936. 1 ex., leg. TE [MIZ]; Chylice [EC07]. date missing. 2 exx., leg. TE [MIZ]; Warszawa-Wilanów [EC07]. 27 VIII 1915. 1 ex., leg. TE [MIZ]; Warszawa-Natolin [EC07], 22 VI 1888, 24 IV 1893. 19 V 1897. 4 exx., leg. WM [USMB]: Warszawa-Saska Kępa [EC08]. 26 IV 1916, 1 ex., leg. TE [MIZ]; Warszawa-Zacisze [EC09]. 7 V 1916. 1 ex., leg. TE [MIZ]; Pustelnik [EC09], 19 X 1948, 1 ex.. leg. GO [MIZ]; Drewnica [EC09]. 8 VI 1917, 1 ex.. leg. TE [MIZ]: Warka [EC13]. 22 VI 1952, 1 ex.. leg. BB [MIZ]: Baniocha [EC16], 17 V 1948, 1 ex., leg. RB, 24 V 1951, 1 ex., leg. JF [MIZ]; Świder [EC17]. 18 IV 1950, 2 exx., leg. RB [MIZ]; Jabłoń [EC17], 1 VII 1908, 1 ex., leg. WM [USMB]; Miłosna [EC18]. 4 VI 1897. 1 ex.. leg. WM [USMB]: Warszawa-Goclawek [EC18]. 18 IV 1948, 6 exx.. leg. GO \& RB [MIZ]: Warszawa-Wawer [EC18], 27 VI 1890 and 27 VI 1895. 2 exx., leg. WM [USMB]; Warszawa-Anin [EC18], 27 IX 1947, 1 ex., leg. RB. III 1949, 3 exx., leg. WD, 29 V 1950. 5 exx.. leg. BB [MIZ]: Celestynów [EC26]. 9 IV 1949 and 9 IV 1951. 2 exx.. leg. MZ [MIZ]: Śródborów [EC27]. $20 \mathrm{~V}$ 1927, 16 exx., leg. TE [MIZ]: Dębe Wielkie [EC38], 15 VI 
1919, 1 ex., leg. TE [MIZ]; Trąbki [EC45], 6 V 1892, 1 ex., leg. WM [USMB]; Mienia [EC47]. 14 V 1933, 1 ex.. leg. AB [MIZ]: Struga [ED00]. 5 V 1922, 1 ex.. leg. TE. 2 X 1958. 2 exx., leg. BB [MIZ]: Czarma Struga [ED00], 3 V 1898, 12 IV 1910, 17 IV 1922. 16 IV 1924, 18 exx., leg. TE, 7 IV and 22 IX 1949, 6 exx., leg. RB [MIZ]; Zegrze [ED01], 20 III 1937, 2 exx., leg. AB, 14 III 1948, 1 ex., leg. RB, 22 III 1949, 19 III 1953 and 18 IV 1956, 3 exx., leg. MZ [MIZ]; Popowo [ED02] ad Serock. 24 V 2003. 1 ex.. leg. WJ [ISEA]: Pultusk [ED04]. 17 V 1929. 3 exx.. leg. TE [MIZ]; Kalinowo [ED65], 6 VII 1933, 1 ex., leg. AB [MIZ].

Podlasie Lowland: Mocarze [EE90] ad Jedwabne. 24 V 1969, 4 exx.. leg. JP [ISEA]; Międzyrzec Podlaski [FC26]. date missing. 2 exx.. leg. EI [MIZ]; Niemirów [FC49], 12 V 2003. 1 ex., leg. DK [ISEA]; Mielnik [FD30], 4 VI 2002, 1 ex.. leg. MM [ISEA].

Bialowieża Primeval Forest: Białowieża [FD94], 29 V 1984, 1 ex.. leg. JG [USMB].

Lower Silesia: Trzebień [WS39]. 19 V 2007. 1 ex.. leg. RD [USMB].

Trzebnickie Hills: Siemianice [BB97] ad Kępno, 12 VI 1982, 1 ex.. leg. PS [ISEA]; Oborniki Śląskie [XS39]. 10 VI 1977. 1 ex.. leg. JL [USMB].

Upper Silesia: Dziergowice (Dziergowitz) [CA06], 4 VII 1929. 2 exx.. leg. HN [USMB]; Rybnik-Paruszowiec (Paruschowitz) [CA25], 5 V 1912, 2 exx., leg. SC [USMB]; GliwiceBrzezinka (Birkenau) [CA37], 9 IV 1936, 2 exx.., leg. KT [USMB]; Gliwice-Labędy (Laband) [CA38]. 19 IX 1938, 2 exx.. leg. HN [USMB]; Tarnowskie Góry-Pniowiec (Pniow) [CA49]. 1 VI 1934, 1 ex.. leg. KI [USMB]: Bytom (Beuthen) [CA58], 4 V 1920 and 24 IV 1932, 3 exx.. leg. KI [USMB]: Chełmek [CA75]. 12 VI 1932, 25 VI 1939, 3 exx., leg. SS \& SP [ISEA]: Jaworzno-Szczakowa [CA76]. 2 X 1932. 4 exx.. leg. EM [MIZ]; Żarki [CA84] ad Chrzanów. 5 V 1882. 5 exx., leg. SS [ISEA]: Babice: Lipowiec [CA84], 5 V 1882, 1 ex., leg. SS [ISEA]: Klucze: Pustynia Błędowska [CA97]. 3 V 1932. 2 exx., leg. EM [MIZ], 8 VI 1971, 1 ex., leg. JP [ISEA]: Lubliniec [CB31], 1 VI 1993. 1 ex.. leg. RD [USMB].

Kraków-Wieluń Upland: Klucze [CA97] ad Olkusz, 3 VI 1998, 1 ex.. leg. DK [ISEA]; Klobuck [CB54], 9 VI 1924, 1 ex., leg. HC [MIZ]; Kraków-Zakrzówek [DA14], ca. 1900, 3 exx., leg. MR. 3 IV 1937, 4 exx.. leg. SP [ISEA]; Kraków-Pychowice [DA14], 8 VI 1919. 6 V 1937, 17 exx.. leg. SS \& SP [ISEA]: Kraków-Podgórki [DA14]. 28 V 1954, 6-20 IV 1971. V 1985, 42 exx.. leg. WS, ZS. MO \& JM [ISEA]: Kraków-Wola Justowska [DA14]. 19 V 1878. 1 ex., leg. SS [ISEA]; Kraków-Olsza [DA24], 6 V 1874, 15 exx., leg. BK [ISEA].

Malopolska Upland: Wymyslów [CC82] ad Pabianice, 12 V 1912, 1 ex.. leg. EI [MIZ]; Szczepanowice [DA37] ad Miechów, 9 V 1994. 1 ex., leg. DK [ISEA]; Grabowiec nat. res. [DA69], 18 VI 1997, 3 exx.. leg. RD [USMB]: Kików [DA98], 19 VI 1995, 19 exx., leg. RD \& WZ [USMB]: Nasilów [EB68] ad Kazimierz Dolny, 8 VII 2003, 1 ex., leg. MM [ISEA].

Świętokrzyskie Mts.: Dyminy [DB73], 27 V 1952, 1 ex., leg. JF [MIZ].

Lubelska Upland: Opole Lubelskie [EB66], 23 V 1915. 1 ex.. leg. EI [MIZ]; Kazimierz Dolny [EB68]. 3 V 1923, 2 exx., leg. TE [MIZ]: Pulawy [EB69]. 24 IV 1925, 4 exx.. leg. HC [MIZ]; Tyszowce [FB91] ad Hrubieszów. 30 VI 1985, 3 exx., leg. MM [ISEA]: Gródek [GB03] ad Hrubieszów. $24 \mathrm{~V}$ 1987, 1 ex., leg. MM [ISEA].

Roztocze Upland: Zwierzyniec [FB30]. V 1906. 4 exx., leg. FF [MIZ]; Florianka [FB30]. 15 V 1913, 5 exx., leg. FF [MIZ].

Sandomierska Lowland: Niepolomice [DA44]. 17 V 1989. 3 exx., leg. DK [ISEA]; Staniątki [DA44]. 30 III 1913. 2 exx., leg. EM [MIZ]; Puszcza Niepolomicka Forest: Klaj [DA53]. 1904. 10 exx.. leg. MR [ISEA]; Padew Narodowa [EA38], 14 VI 1931, 4 exx.. 1eg. SS [ISEA]; Stalowa Wola [EB70]. VIII 1950, 1 ex., leg. WE [MIZ]; Bobrówka [FA34] ad Jarosław, 15 VII-15 VIII 1960, 6 exx.. leg. SO [USMB]; „Puszcza Niepolomicka Forest”. 6 VI 1912 and 14 VI 1914. 2 exx., leg. TE [MIZ]. 
Western Beskidy Mts.: Skawina [DA13], 2-16 VI 1932, 7 exx., leg. SS [ISEA]; KrakówBorek Fałęcki [DA23]. IV-V 1874, 2 exx., leg. BK. 6 VI-6 X 1931. 23 IV-4 VI 1932, 96 exx.. leg. SS, 6 V 1934. 2 exx., leg. EM [ISEA], 28 V 1933, 12 exx., leg. EM [MIZ]: Wieliczka: Las Krzyszkowicki forest [DA23], 6 IV 1878, 4 exx., leg. BK [ISEA].

M. tibialis (FABR.) consists of two subspecies: M. tibialis tibialis, found almost everywhere in Europe, and M. tibialis subaereus REITTER, 1904, known only from Kirghistan and Kazakhstan. In view of data from many recent publications (among others, KuBIsz \& Tsinkevich 2001; Gutowski et al. 2005; Gutowski et al. 2006; Przewoźny 2007; Ruta 2007; JAskula et al. 2009; GuTOWski et al. 2010) and from labels on specimens deposited in the studied collection, the statement from KFP: ,... reported from few localities in Poland..." is no longer true. It is one of the most commonly captured Tenebrionidae beetles. It is widespread all over Poland. Specimens coming from the reviewed collections and captured in Podlasie Lowland, Trzebnickie Hills, Lower Silesia and Western Beskidy Mountains give strong support for including these regions (not listed in KFP) to the range of distribution of this species in Poland.

\section{ALLECULINAE LAPORTE, 1840}

\section{Alleculini LaPORTE, 1840}

\section{Allecula morio (FABRICIUS, 1787)}

Wielkopolska-Kujawy Lowland: Nowa Sól (Neusalz) [WT43]. 14 VII 1906, 1 ex.. coll. MIZ; Karszew [CC57] ad Dąbie, 5 VII 2000, 1 ex., leg. RM [ISEA].

Mazowiecka Lowland: Podkowa Leśna [DC87]. 15-29 VI 1934. 9 exx.. leg. TE [MIZ]; Warszawa-Buchnik [DC99], 19 VII 1956. 7 exx., leg. ZI. 23 III 1960, 1 ex., leg. BB [MIZ]: Warszawa-Mlociny [DC99], 29 XI 1953. 2 exx.. leg. BB [MIZ]; Warszawa-Bielany [DC99]. 18-27 VI 1888, 3 exx., leg. WM [USMB], 5 VII 1947, 3 exx., leg. ZW, 23 XII 1951, 26 IX 1954. 30 I 1955, 16 V 1958, 31 V 1961, 11 XII 1964, 6 VII 1965, 12 V 1969, 15 exx.. leg. BB [MIZ]: Piaseczno [EC06], 8 VII 1906, 3 exx., leg. WM [USMB]; Warszawa-Natolin [EC07], date missing, 3 exx., leg. TE [MIZ]; Warszawa-Morysinek [EC07]. 8 VI 1956, 2 exx.. leg. BB [MIZ]; Warszawa-Łazienki [EC08], 23 III 1977, 1 ex.. leg. BB [MIZ]; Warszawa-Agrykola [EC08]. 2-10 III and 2 VI 1959, 3 exx., leg. BB [MIZ]; Świder [EC17]. 28 VII 1900, 2 exx., coll. MIZ: Świdry Male [EC17]. 14 VI 1964. 1 ex., leg. BB [MIZ]: Urle [ED41]. 30 IV 1967. 1 ex.. leg. BB [MIZ].

Bialowieża Primeval Forest: Bialowieski P. N.. f. comp. 340 [FD94], 19 IV 1967. 2 exx.. leg. BB [MIZ]; ad Białowieża [FD94], 16 VII 1992, 1 ex., leg. JG [ISEA].

Lower Silesia: Ligota Wielka (Ellguth Ottmachau) [XR49] ad Otmuchów, VIII 1909 and 7 VII 1926, 3 exx., leg. ED [USMB]: Wroclaw: Park Szczytnicki [XS46]. 19 VI 1970, 3 exx.. leg. BB [MIZ]; Borowa Oleśnicka [XS57], 23 VI 1977. 1 ex.. leg. BB [MIZ].

Upper Silesia: Pokój (Carlsruhe O/S) [XS94], 10 VI 1928. 2 exx.. leg. HN [USMB]; Zaborze [CA74] ad Oświęcim, 5-6 VIII 1902, 1 ex.. leg. SS [ISEA]; Chełmek [CA75], 15-23 VII 1878. 2 exx.. leg. SS [ISEA]; Babice: Lipowiec castle [CA84], 29 V 1882, 1 ex.. leg. SS [ISEA]; Żarki 
[CA84] ad Chrzanów. 16-20 VI 1881, 1 ex.., leg. SS [ISEA]; Bolęcin [CA95], 12 VI 1902, 1 ex., leg. SS [ISEA].

Kraków-Wieluń Upland: Czatkowice [DA05] ad Krzeszowice, 9 VII 1903, 1 ex.. leg. SS [ISEA]: Kraków-Las Wolski [DA14], 14 II 1957. 4 exx., leg. BB [MIZ]: Ojców [DA16], date missing. 1 ex.. leg. EI, 3 VIII 1907, 1 ex.. leg. TE [MIZ]. 13-17 VII 1966, 8 VII 1967, 5 exx., leg. JP [ISEA]; Kraków-Śródmieście [DA24], 28 VII 1985, 1 ex., leg. DK [ISEA]; Kraków [DA24]. 1914, 1 ex., leg. TE [MIZ].

Świętokrzyskie Mts.: Nowa Slupia [EB03]. 11 V 1956, 12 exx.. leg. BB [MIZ].

Lubelska Upland: Kazimierz Dolny [EB68]. V 1934, 1 ex., leg. TE [MIZ]; Pulawy [EB69], 2 VII 1953. 1 ex.. leg. BB [MIZ].

Roztocze Upland: Florianka [FB30], date missing. 1 ex., leg. FF [MIZ]; Zwierzyniec [FB30]. 4 VIII 1911, 1 ex., leg. TE [MIZ].

Sandomierska Lowland: Tarnów [DA94]. 1892, 8 exx.. leg. MR [ISEA]; Jaroslaw [FA24]. 20-30 VI 1888, 1 ex.. leg. SS [ISEA]; Księżpol [FA28]. 25 VII 1911. 1 ex.. leg. TE [MIZ].

Western Sudety Mts.: Jedlina-Zdrój (Charlottenbrunn) [WS91]. VIII 1917. 1 ex.. leg. ZM. 10 VI 1928, 2 exx., leg. HN [USMB].

Western Beskidy Mts.: Ustroń [CA40]. 1873, 1 ex., leg. BK [ISEA]; Polanka Wielka [CA73] ad Oświęcim. 6 VII 1900, 14 exx., leg. SS [ISEA]; Beskid Śląski. Czarna Wiselka Valley [CV59]. 19 VI 1962, 1 ex., leg. BB [MIZ]; Zawoja [CV99], date missing. 1 ex., leg. EM [MIZ]; Skawica [DA00]. 7 VII 1983, 11 VIII 1996, 26 VII 2002, 3 exx.. leg. ST [ISEA]: Skomielna Czarna [DA10], VII 1934, 1 ex., leg. EM [MIZ]; Skawina [DA13], 4 VIII 1986, ad lucem, 1 ex.. leg. WI [ISEA]: Kraków-Wróblowice [DA23]. budynek mieszkalny, 15 VIII 1998. 17 VII 2003, 25 VII 2005, 3 exx., leg. DK [ISEA]: Gorzków [DA32]. 1 VII 1920. 1 ex.. leg. EM [MIZ]: Sieraków [DA32] ad Dobczyce. 20 VI 1909, 2 exx.. leg. SS [ISEA]; Mordarka [DA60] ad Limanowa, 16 VII 1903, l ex., leg. SS [ISEA]; Paleśnica [DA81] ad Zakliczyn. 1987, 30 VIII 1989, 6 exx., leg. JM [ISEA]; Zakliczyn [DA82]. VII 1983, 1 ex., leg. JM [ISEA]; Ponice [DV29] ad Rabka. 30 VII 1911, 1 ex.. leg. SS [ISEA]; Rytro [DV78], 22 VII 1899. 15 VII 1901, 2 exx.. leg. SS [ISEA].

Nowotarska Dale: Poronin [DV26], 19 VII 1949, 1 ex., leg. AR [MIZ].

Eastern Beskidy Mts.: Jasło [EA31]. VII 1921, 1 ex., leg. KA [ISEA]; Krasiczyn [FA11]. 22 IX 1962, 1 ex., leg. BB [MIZ].

Tatry Mts.: Czerwony Stawek [DV25], 23 VII 1904, 1 ex., leg. SS [ISEA].

A species widespread in central Europe. Probably living in all KFP regions in Poland. Specimens deposited in ISEA confirm the possibility of capturing beetles of this species even in lower mountain localities (Tatry Mountains). It is a saproxylophage, comparatively rarely captured due to its nocturnal activity pattern. In MIZ collections, there are no voucher specimens for notes signed „B. Burakowsk **” (KFP) from the following KFP regions: Baltic Coast, Pomeranian Lake District, Lower Silesia, Western Sudety Mts.

\section{Allecula rhenana $\mathrm{B}_{\mathrm{ACH}}, \mathbf{8 5 9}$}

Wielkopolska-Kujawy Lowland: Otyń [WT44], 22 IV 1971, 2 exx.., leg. BB [MIZ]; Rawicz (Rawitsch) [XT22], VII 1903, 1 ex.. leg. FK [MIZ].

Mazowiecka Lowland: Warszawa-Mlociny [DC99]. 29 XI 1953 and 11 XII 1955. 23 exx.. leg. BB [MIZ]: Warszawa-Bielany [DC99], 7 III 1954 and $10 \mathrm{~V}$ 1962, 4 exx., leg. BB [MIZ]: Warszawa-Morysinek [EC07], 31 I 1960, 26 V 1968, 19 IV 1970, 11 exx.. leg. BB [MIZ]: 
Warszawa-Ogród Saski [EC08], 13 VIII 1974 and 8-28 VII 1975, 3 exx.. coll. MIZ; Klembów [ED20], 13 V 1958, 1 ex.. leg. BB [MIZ].

Świętokrzyskie Mts.: Łysa Góra Mt. [EB03]. 27 X 1968, 2 exx.. leg. BB [MIZ].

Lubelska Upland: Pulawy [EB69], 22 VII 1951, 1 ex., leg. BB [MIZ].

Sandomierska Lowland: Leżajsk [FA06]. 20 IX 1957, 1 ex., leg. BB [MIZ]. [MIZ].

Western Beskidy Mts.: Wisla-Glębce [CV49]. 22 VI 1967 and 25 VI 1968. 4 exx.. leg. BB

A species very close to A. morio in terms of distribution and biology. As it is not so often captured, its occurrence is poorly documented in publications for most of the territory of Poland. Faunistic data on labels of specimens deposited in MIZ, collected by B. BURAKOWSKI, confirm its occurrence in 6 KFP regions. The seventh region, Eastern Beskid Mountains, has been listed in KFP mainly on the basis of original data from publications by TRELLA (1923).

\section{Gonodera luperus luperus (HERBST, 1783)}

Baltic Coast: Międzyzdroje [VV67]. 30 V 1992, ad lucem. 1 ex.. leg. MA [ISEA].

Pomeranian Lake District: Bielinek nad Odrą [VU46], 31 V 1984, 3 VI 1985, 29 V 1988. 13 exx., leg. PS \& LB [ISEA]; Szczecin (Stettin) [VV62], date missing. 1 ex.. coll. MIZ.

Wielkopolska-Kujawy Lowland: Goluchów [YT05]. 23 V 1984, 1 ex.. leg. AM [ISEA]; Kościelec [CC38] ad Kolo, 15 V 1999. 3 exx., leg. MK [ISEA].

Lower Silesia: Strzegom (Striegau) [WS94], V 1924, 1 ex., leg. ED [USMB].

Upper Silesia: Babice: Lipowiec castle [CA84]. 5 V 1882, 1 ex.. leg. SS [ISEA]; Pogorzyce [CA85] ad Libiąż, 8 VI 1889. 1 ex.. leg. SS [ISEA].

Kraków-Wieluń Upland: Klucze [CA97]. 22 VI 1994. 1 ex., leg. DK [ISEA]; Zloty Potok [CB81]. 25 V-26 VI 1900, 3 exx., leg. TE \& FF [MIZ], 24 V 1900, 4 exx., leg. WM [USMB]: Kraków-Bielany [DA14], 5 VI 1879, 1 ex., leg. SS [ISEA]; Ojców [DA16], 26 VI 1964, 14 V 1968, 2 V 1990, 3 exx.. leg. JP [ISEA].

Malopolska Upland: Uniejów-Rędziny [DA28] ad Miechów. 1 VI 1954, 9 VI 1955, 18 V 1966. 5 exx.. leg. WS [ISEA]: Lisiniec [DA37] ad Miechów. 16 VI 1953, 1 ex.. leg. WS [ISEA]: Marchocice [DA47] ad Miechów, 5 VI 1955. 1 ex., leg. WS [ISEA].

Świętokrzyskie Mts.: Kielce [DB73]. 8 VI 1913, 1 ex., leg. EI [MIZ].

Lubelska Upland: Kazimierz Dolny [EB68]. 12 VI 1957, 1 ex.. coll. MIZ.

Western Sudety Mts.: Duszniki-Zdrój (Bad Reinerz) [WR98]. VI 1912, 1 ex., leg. ED [USMB].

Western Beskidy Mts.: Rytro [DV78]. 16-18 VI 1892. 1 ex.. leg. SS [ISEA].

Eastern Beskidy Mts.: Manasterzec [EV98]. Góra Sobień, 8 V 1974, 1 ex., leg. AJ [MIZ].

Pieniny Mts.: Potok Pieniński Valley [DV57]. 28 V 1912, 1 ex.. leg. TE [MIZ]; Krościenko [DV57], 19 VII 1939, 1 ex., leg. TE [MIZ].

In 1944, MAR̂A described a subspecies of G. luperus hellenica (Greek endemic taxon). For this reason, a nominotypical subspecies of G. luperus luperus (HERBST, 1783) occurs in Poland. It is widespread in whole Europe. It has been rarely and sporadically captured in Poland, so there are few publications (KLASINSKI 2007). In ISEA collections, there are specimens coming from Małopolska Upland and Świętokrzyskie Mountains, 
not listed as its regions in KFP. In MIZ collection, there are no voucher specimens for reports from Lower Silesia, signed „B. BuRAKOWSKI*”.

\section{Hymenalia rufipes (Fabricius, 1792)}

Baltic Coast: Świnoujście [VV57], 19 VII 1990. 2 exx.. leg. MA [ISEA]; Międzyzdroje [VV67]. VIII 1948. 1 ex.. leg. KA [ISEA].

Pomeranian Lake District: Bielinek nad Odra [VU46], 18 VII 1987. 1 ex., leg. PS [ISEA]; Szczecin (Stettin) [VV62], date missing. 4 exx.. coll. MIZ.

Wielkopolska-Kujawy Lowland: Mosina [XT29]. 19 VI 1975, 1 ex., leg. MB [ISEA]; Tarnowa [CC37] ad Turek. 25 VI 2001. 1 ex.. leg. MK [ISEA]: Borek [CD18] ad Toruń. 25 VI 1964. 5 exx.. leg. WS [ISEA]: Solec Kujawski [CD 18], 22 VI 1995, 1 ex.. leg. JH [ISEA].

Mazowiecka Lowland: Jablonna [DD90]. 17 VI 1893. 1 ex.. leg. WM [USMB].

Podlasie Lowland: Niemirów [FC49]. 15 VI 2003. 1 ex.. leg. DK [ISEA]; Oslowo [FD30] ad Mielnik. 15 VI 2003, 1 ex.. leg. DK [ISEA]; Mielnik [FD30]. Uszeście nat. res.. 6 VI 1963 and 7-8 VIII 1968, 2 exx.. leg. WS, 22 VI 2001, 2 exx.. leg. DK. 16 VI 2003, 1 ex.. leg. WA [ISEA].

Upper Silesia: Szymiszów (Schemischow) [CB00]. 15 VII 1932. 1 ex.. leg. HN [USMB].

Kraków-Wieluń Upland: Radwanowice [DA05] ad Kraków. 3 VII 1997, 1 ex.. leg. TW [ISEA]; Kraków-Krzemionki [DA14], 17-28 VI 1939, 2 exx.. leg. SP [ISEA]; Kraków-Podgórki [DA14], 12 VII 1967, 1 ex.. leg. WS [ISEA]; Kraków-Pychowice [DA14], 15 VI 1985, 1 ex., leg. DK [ISEA]: Ojców [DA16]. 7 VII 1967, 1 ex.. leg. JP [ISEA].

Malopolska Upland: Krzyżanowice [DA68]. 9 VII 1953. 2 exx.. leg. BB [MIZ]; Skowronno [DA69]. 17 VI 1995, 1 ex.. leg. RD [USMB]: Skotniki Górne [DA78]. 14 IV 1954. 4 exx.. leg. BB [MIZ]; Grabowiec nat. res. [DA79], 9 VI 1954, 1 ex.. leg. BB [MIZ]; Chotel Czerwony [DA88]. 21 V 1952 and 21 V 1958, 6 exx., leg. BB [MIZ]: Sandomierz [EB51]. 13 VII 1950. 2 exx.. leg. MZ [MIZ].

Lubelska Upland: Opoka Duża [EB63], 26 VI 1962. 1 ex.. leg. WS [ISEA].

Pieniny Mts.: Biale Skały [DV57]. 9 VI 1972, 1 ex., leg. BB [MIZ].

The species inhabits mainly southern Europe, and is locally found in xerothermic habitats in its northern part, including Poland. The larvae develop in soil among roots of grasses and perennial plants (they are particularly connected with field wormwood, Artemisia campestris (L.)). The beetle is rarely captured in Poland, but its occurrence has been confirmed by quite recent publications (RUTA 2007; BYK 2001; KONWERSKi \& PRZEWOŹNY 2001). In KFP, the species has not been reported from north-western part of Poland and from Bieszczady and Tatry Mountains. The ISEA collection contains specimens from Podlasie Lowland and Lubelska Upland. In MIZ collection, there are voucher specimens for notes of B. BuRakowski* from Malopolska Upland and Pieniny Mountains; the specimen from Międzyzdroje is deposited in ISEA collection.

\section{Hymenophorus doublieri Mulsant, 1851}

(Fig. 10)

Bialowieża Primeval Forest: f. comp. 471 [FD84]. 19 XI 1959. 1 ex. ex larva 25 V 1960. leg. BB [MIZ]. 
A southern-European species, with scarce relict localities in central Europe and in southern provinces of Sweden and Finland, also reported from Siberia. It feeds in old trunks of rotten pine trees. Recorded in Poland on the basis of specimens captured more than a hundred years ago in Masuria. The authors of KFP stressed the necessity of confirmation of its occurrence in Poland. During review of MIZ collection (for the present publication), one beetle specimen was identified as $H$. doublieri. It was grown from larval stage by B. BuRAKOwski in 1960, i.e., 27 years before publication of Vol. 14 of KFP. The species has been placed in the Polish Red List (PAwŁowski et al. 2002), CR category; this has appeared to be right and reasonable.

\section{Isomira murina murina (LinNaEus, 1758)}

(Fig. 9)

Pomeranian Lake District: Cedynia [VU46]. 10 VI 2003, 1 ex.., leg. DK [ISEA]; Bielinek nad Odra [VU46]. 27 VI 1959. 1 ex.. leg. BB [MIZ]. 22 VI 1984. 3 exx.. leg. LB [ISEA]: Grędziec [VU99] ad Pyrzyce, 11 VI 2003, 1 ex., leg. DK [ISEA]; Stary Przylep [WU09] ad Pyrzyce, 11 VI 2003. 6 VI 2008, 2 exx.. leg. DK [ISEA]: Wolsko [XU38] ad Miasteczko Krajeńskie. 17 V 2002. 1 ex., leg. MM [ISEA]; Bielawy [XU69] ad Naklo, 13 VI 2003, 1 ex.., leg. DK [ISEA]: Ślesin [XU79]. 16 V 1990. 5 exx.. leg. JP [ISEA]: Grabowo [CE10]. Kozielec nat. res., 8 VI 1994. 1 ex., leg. MS [ISEA]; Topolno [CE11] ad Gruczno. 30 V 1996, 1 ex.. leg. DK [ISEA]; Unislaw [CE20]. 16 V 2002. 1 ex.. leg. DK [ISEA]: Wzgórza Plutowskie Hills [CE20]. 24 VI 1964. 4 VI 1965, 4 exx.. leg. WS. 27 V 1996, 1 ex., leg. DK [ISEA]: Gruczno [CE21]. 23 V 1995, 1 ex., leg. DK [ISEA]; Starogród [CE21]. Góra św. Wawrzyńca nat. res., 16 V 2002. 1 ex.. leg. DK [ISEA]; Terespol Pomorski [CE22]. 1 VI 1990, 1 ex.. leg. JP [ISEA]; Czarcie Góry nat. res. ad Świecie [CE32], 10 VII 1996, 1 ex., leg. DK [ISEA]: Nowe Marzy [CE42], 25 V 1995, 1 ex.. leg. DK [ISEA]; Tymawa [CE56] ad Gniew, 19 VI 2001, 1 ex., leg. DK [ISEA]; Jaźwiska [CE56] ad Gniew. 20 VI 2001, 1 ex., leg. DK [ISEA]; Gniew [CE56], 10 V 2002, 1 ex.. leg. MM [ISEA]: Biala Góra [CE67] ad Sztum. 19 VI 2001, 1 ex., leg. MM [ISEA].

Masurian Lake District: Nowe Miasto Lubawskie [DE02]. 7 VI 2002, 1 ex.. leg. DK [ISEA]; Krosno [DE49] ad Orneta. 23-25 VI 1982. 8 exx.. leg. BB [MIZ]; Osowiec-Twierdza [FE03], 8 VI 2009, 2 exx.. leg. WJ [ISEA]: Gawrych Ruda [FE38]. 5 VI 2002, 1 ex., leg. MM [ISEA]; Puszcza Augustowska Forest ad Plaska [FE47]. 25 VI 1986. 1 ex.. leg. JG [ISEA].

Wielkopolska-Kujawy Lowland: Laski Lubuskie [VU71] ad Kostrzyn. 9 VI 2003, 1 ex., leg. DK [ISEA]; Owczary [VU71] ad Kostrzyn. 10 VI 2003, 1 ex.. leg. DK [ISEA]; Górzyca [VU71] ad Kostrzyn. 10 VI 2003, 1 ex., leg. DK [ISEA]: Puszczykowo [XT29]. 12 VI 1987. 1 ex., leg. PS [ISEA]: Niwka [XT29] ad Poznań, 15 VI 1975. 6 exx.. leg. MB [ISEA]: Rogalin [XT38], 16-18 VI 1975, 7 exx.., leg. MB [ISEA]; Sycyn Dolny [XU13], 19 V 1993, 1 ex., MA [ISEA]: Ujście [XU18] ad Pila. 18 V 2002. 1 ex.. leg. MM [ISEA]: Kościelec [CC38] ad Kolo. 10 IV 1998, 2 exx.. leg. MK [ISEA]; Solec Kujawski [CD18], 23 VI 1995, 1 ex.. leg. JH [ISEA]: Przypust ad Nieszawa [CD45], 14 VI 2003, leg. MM [SEA]; Włoclawek [CD63], the vicinity of Kulin nat. res.. $13 \mathrm{~V} 2002$. leg. MM [ISEA].

Mazowiecka Lowland: Kampinoski N. P.. f. distr. Józefów [DC69], 12 VII 1899, 2 exx.. leg. WM [USMB], 9 VI 2004, 2 exx.. leg. WJ [ISEA]: Warszawa-Jelonki [DC98], 6 VI 2003. 1 ex., leg. WJ [ISEA]: Warszawa-Bielany [DC99]. 12 VI 1895, 4 exx.. leg. WM [USMB]: Jabłonna [DD90]. 30 VI 1893. 1 ex.. leg. WM [USMB]: Warszawa-Natolin [EC07]. 4 VII 1894 
and 10 VI 1898, 2 exx., leg. WM [USMB]; Świder [EC17], 15 VII 1900, 8 exx.. leg. WM [USMB]; Otwock [EC17], 4 VI 1894. 2 exx., leg. WM [USMB]; Miłosna [EC18], 4 VI 1897. 1 ex.. leg. WM [USMB]: Trąbki [EC45], 6 V 1902, 1 ex.. leg. WM [USMB]: Urle [ED41], 8 VII 1909. 1 ex.. leg. WM [USMB]; Piątnica [ED79] ad Łomża. 15 VI 2003, 1 ex.. leg. WA [ISEA]: „Warszawa”, 3 V 1895 i 10 V 1904, 3 exx.. leg. WM [USMB].

Podlasie Lowland: Stulno [FB89] ad Wlodawa. 16 VI 2003. 1 ex.. leg. DK [ISEA]; Sutno [FC49] ad Mielnik, 22 VI 2001, 1 ex., leg. MM [ISEA]; Niemirów [FC49], 4 VI 2002, 1 ex., leg. DK [ISEA]: Wajków [FC49]. 7 VII 2009, 1 ex.. leg. WJ [ISEA]: Zaczopki [FC68] ad Terespol. 3 VI 2002. 1 ex.. leg. DK [ISEA]: Gródek n/Bugiem [FD01]. 21 VI 2001. 1 ex.. leg. DK [ISEA]: Wólka Zamkowa [FD10] ad Drohiczyn, 21 VI 2001, 1 ex.. leg. DK [ISEA]; Zajęczniki [FD10] ad Drohiczyn. 21 VI 2001, 1 ex., leg. DK [ISEA]; Osłowo [FD30] ad Mielnik, 15 VI 2003, 1 ex., leg. DK [ISEA]; Mielnik [FD30]. 22 VI 2001. 4 VI 2002. 3 exx.. leg. DK [ISEA]: Haćki [FD45] ad Bielsk Podlaski, 5 VI 2002, 1 ex., leg. DK [ISEA]; Dąbrówki [FD49] ad Wasilków. 5 VI 2002. 1 ex., leg. DK [ISEA]; Biebrzański N. P., Carska Droga. f. comp. 20 [FE01], 30 V 2002, 4 exx., leg. WA [ISEA]: Biebrza Valley: Dawidowizna [FE22]. 12 VI 2009, 3 exx.. leg. WJ [ISEA]: Studzianki [FE40] ad Wasilków, 5 VI 2002, 1 ex.. leg. DK [ISEA].

Bialowieża Primeval Forest: f. comp. 259C [FD95], 6 VII 1994, 1 ex., leg. LB [ISEA].

Lower Silesia: Ligota Wielka (Ellguth Ottmachau) [XR49] ad Otmuchów. VI 1919. VI 1921, 13 VI 1926, 3 exx., leg. ED [USMB].

Upper Silesia: Slawięcice (Slawentzitz) [CA18]. 4 VII 1926, 1 ex.. leg. KI [USMB]; Zbroslawice (Broslawitz) [CA48], 11 VI 1926, 3 exx.. leg. HN [USMB]; valley of Drama river ad Repty [CA48], 11 VI 1926, 9 exx., leg. KI [USMB]: Bytom (Beuthen) [CA58], 29 VI 1926. 1 ex., leg. KI [USMB]; Chełmek [CA75], 12 VI 1900, 13 VII 1902, 12 VI 1932, 3 exx., leg. SS [ISEA]: Babice [CA84], 24 VI 1882, 3 exx.. leg. SS [ISEA]: Żarki [CA84] ad Chrzanów. 16-20 VI 1881, 1 ex.. leg. SS [ISEA].

Kraków-Wieluń Upland: Niegowonice [CA88] ad Łazy, 17 VI 1998, 1 ex., leg. DK [ISEA]: Syborowa Góra [CA97] ad Olkusz. 2 VII 1997. 23 VI 1998. 2 exx., leg. DK [ISEA]: Pomorzańskie Skalki nat. res. [CA97] ad Olkusz, 2 VII 1997, 23 VI 1998. 2 exx., leg. DK [ISEA]; Klucze [CA97] ad Olkusz, 3 VI 1998, 1 ex.. leg. DK [ISEA]; Rzędowa Skala rock [CA99] ad Zawiercie. 5 VII 1997, 17 VI 1998, 2 exx., leg. DK [ISEA]: „Biakło” rock ad Olsztyn [CB72], 17 VI 2004, 1 ex., leg. JP [ISEA]: Czernichów [DA03], 12 VI 1991, 3 exx.. leg. JP [ISEA]: Przeginia Duchowna [DA04]. 29-31 V 1906, 15 VI 1931, 3 exx., leg. SS, 6 VI 2009. 1 ex., leg. DK [ISEA]; Krzeszowice [DA05], 10 VII 1902, 2 exx., leg. SS [ISEA]; Sułoszowa [DA06], 21 VI 1990, 1 ex., leg. JP [ISEA]: Smoleń [DA08]. 4 VII 1997. 2 VI 1998, 2 exx.. leg. DK [ISEA]; Kraków-Sikornik [DA14], 26 V-12 VI 1936, 51 exx.. leg. SP [ISEA]; KrakówPrzegorzały [DA14], 19 V 1918, 21 V 1923, 3 exx., leg. SS [ISEA]; Kraków-Bielany [DA14]. 7 VI 1967, 1 ex., leg. WS [ISEA]; Kraków-Zakrzówek [DA14]. 14 VI 1906. 16 VI 1919. 2 exx.. leg. SS. 17 VI 1939, 2 exx., leg. SP [ISEA]; Kraków-Pychowice [DA14]. 23 VI-13 VII 1919. 2 exx., leg. SS, 18 VI 1984, 12 VI 1987, 6 exx.. leg. DK [ISEA]: Kraków-Podgórki [DA14]. $10 \mathrm{~V} 1947.1$ ex.. leg. JS. 22 VI 1965, 2 exx.. leg. WS [ISEA]: Piekary [DA14]. 11 VI 1991. 2 exx., leg. JP [ISEA]; Ujazd [DA15], 7 VII 1919, 1 ex., leg. SS [ISEA]; Modlnica [DA15]. 4 VI 1907, 1 ex., leg. SS [ISEA]; Wierzchowie [DA15], 15 VII 1923, 4 exx.., leg. SS [ISEA]: Prądnik Korzkiewski [DA15]. 29 VI 1990. 1 ex.. leg. MM [ISEA]; Bolechowice [DA15]. 19 VII 1919. 1 ex.. leg. SS [ISEA]; Będkowska Valley [DA15] ad Kraków, 13 VI 1969. 1 ex.. leg. WS [ISEA]; Kluczwody Valley [DA16] ad Kraków, 5 VI 1968, 3 exx.. leg. WS [ISEA]; Jerzmanowice [DA16]. rock $502 \mathrm{~m} .15$ VI 1969. 1 ex.. leg. WS (determined as Isomira icteropa (KusT.)!) [ISEA]; Ojców [DA16], 19-31 V 1990, 2 exx., leg. JP [ISEA]: Kraków-Olsza [DA24]. 1873. 
1 ex.. leg. BK, 28 VI 1911. 1 ex.. leg. SP [ISEA]; Kraków-Prądnik Czerwony [DA24]. 2 VI 1984. 4 exx., leg. MM [ISEA]; Kraków-Prądnik Biały [DA24], 11 V 1985. 13 VI 1987, 6 exx., leg. MM [ISEA]; Kraków-Mydlniki [DA24]. 1 ex., leg. MM [ISEA]; Kraków-Podgórze [DA24]. VI 1916, 1 ex., leg. SP [ISEA].

Malopolska Upland: Łódź-Łagiewniki [CC94], 25 VI 1997, 1 ex., leg. WZ [USMB]; Jaksice [DA18] ad Miechów. 31 V 1991. 1 ex.. leg. MM [ISEA]; Szczepanowice [DA37]. 17 VI 1994, 1 ex.. leg. MM [ISEA]; Dale [DA47] ad Raclawice, 16 VI 1954, 8 VI 1965, 3 exx., leg. WS [ISEA]; Morsko [DA65] ad Koszyce, 31 V 1991. 2 exx.. leg. JP \& MM [ISEA]; Polana Polichno nat. res. [DA69]. 15 VI 1995 and 15 VII 1996. 3 exx. leg. RD [USMB]: Malkowice [DA76] ad Koszyce, 31 V 1991, 12 exx., leg. JP \& MM [ISEA]: Skorocice [DA78]. 23 VI 1959, 4 VII 1961. 13 VI 1964, 16 VI 1965, 10 exx., leg. WS, 18 V 1992, 3 exx., leg. JP [ISEA]: Galów [DA79] ad Pińczów. 26 V 1993. 1 ex.. leg. MM [ISEA]; Bogucice [DA79] ad Pińczów. 26 VI 1953. 22 VI 1962, 9 exx., leg. WS [ISEA]; Grabowiec nat. res. [DA79]. 9 VI 1954. 9 exx.. leg. BB [MIZ]; Chotel Czerwony [DA88], 28 V-19 VI 1953, 6 exx., leg. WS [ISEA]; Owczary [DA88] ad Busko-Zdrój. 27 V and 9 VI 1999. 2 exx.. leg. DK \& LB [ISEA]: Kików [DA98]. 6 VI-2 VII 1991, 2 exx., leg. MM [ISEA]; Skowronno [DB60], 22 VI 1962, 25 V 1963, 3 exx.. leg. WS, 29 V 1990, 1 ex., leg. AP. 10 VI 1992. 3 exx., leg. JP [ISEA]; Gorzyczany [EB40] ad Sandomierz. 28 V 1971. 3 exx.. leg. WS [ISEA]: Góry Pieprzowe [EB51]. 9 VI 1951. 2 VII 1952. 2 exx.. leg. WS, 8 VI 2004, 1 ex., leg. DK [ISEA]; Dwikozy [EB52] ad Sandomierz, 31 V 1967, 2 exx., leg. WS [ISEA].

Świętokrzyskie Mts.: Zelejowa Góra [DB53] ad Chęciny, 21 VI 1967. 2 exx.. leg. WS [ISEA]: Miedzianka [DB53], 11 VI 1984, 2 exx.. leg. WJ [MIZ]: Radomice [DB72], 21 VI 1983. 1 ex., leg. LI [MIZ]; Kielce [DB73], 1859. 1 ex., leg. AW [ISEA]; Nowa Shupia [EB03], 16 V-24 VI 1966, 2 exx.. leg. WS [ISEA].

Lubelska Upland: Dobre [EB68] ad Kazimierz Dolny, 28 V 2003, 1 ex.. leg. DK [ISEA]; Puławy [EB69]. 23 VI 1956, 1 ex., leg. WB [MIZ]; Łęczna [FB38] ad Lublin, 9 VI 1970, 1 ex., leg. WS [ISEA]; Tarnogóra [FB53] ad Krasnystaw, 3 VI 1971. 2 exx., leg. WS [ISEA]; Gródek [GB03] ad Hrubieszów, 10 VII 1962, 7 VI 1963, 10 VI 1970, 4 VI 1971. 30 VI 1985, 10 exx.. leg. WS, ZS, MO \& MM [ISEA].

Roztocze Upland: Szczebrzeszyn [FB31]. 1 VII 1985, 2 exx.. leg. DK [ISEA].

Sandomierska Lowland: Tarnów [DA94], 15 III 1891, 1 ex., leg. SS [ISEA].

Eastern Sudety Mts.: Mielnik [XR17] ad Klodzko, southern slope of Krowiarki, 19 VI 1980, 1 ex.. leg. AK [MIZ]; Gluchołazy (Bad Ziegenhals) [XR67]. 26 VI 1927, 2 exx., leg. KI [USMB].

Western Beskidy Mts.: Skawica [DA00], 20 VI 1985, 1 ex., leg. ST [ISEA]; Nowy Sacz [DV79], 13 IV 1892, 2 exx., leg. SS [ISEA].

Eastern Beskidy Mts.: Babice [FA01] ad Krzywcza. 23 V 1989. 1 ex.. leg. JP [ISEA]; Przemyśl: Winna Góra [FA21], 27 VI-2 VII 1881, 2-13 VII 1884, 8 exx.. 1eg. BK \& SS, 26 V 1989. 2 exx., leg. MM [ISEA]; Przemyśl: Ostrów [FA21]. 16 VI-3 VII 1881, 15 exx., leg. BK [ISEA]; Przemyśl: Lipowica [FA21], 22-23 VI 1881, 9 exx., leg. BK [ISEA]; Przemyśl: Wzniesienie [FA21], 11 VII 1884. 1 ex., leg. BK [ISEA]; Łuczyce [FA31] ad Przemyśl. 24 VI 1965, 1 ex., leg. WS [ISEA].

Pieniny Mts.: Czorsztyn [DV47]. VI 1951. 2 exx., leg. KA. 25 V 1961. 19 VI 1967. 3 exx.. leg. WS, 17 VI 1992. 2 exx.. leg. SK [ISEA]: Ostra Skala [DV57]. 19 VI 1957. 1 ex.. leg. WS [ISEA]: Pieninki [DV57]. 21 VI 1957, 1 ex., leg. WS [ISEA]; Pulsztyn Mt. [DV57], 6 VI 1964. 2 exx.. leg. WS [ISEA]; Długi Gronik [DV57]. 6 VI 1964. 3 exx.. leg. WS [ISEA]; Zamczysko Mt. [DV57], 19 VI 1967, 2 exx., leg. WS [ISEA]; Grabczycha [DV57], 13 VI 1972, 12 IV 1973. 
18-22 VI 1973 and 1 VII 1974, 12 exx., leg. BB [MIZ]; Wąwóz Sobczański [DV57]. 26 V 1973. 4 exx., leg. BB [MIZ]: Biale Skaly [DV57], 9 VI 1972, 1 ex.., leg. BB [MIZ]: Trzy Korony [DV57], 17 VI 1972. 3 exx.. leg. BB [MIZ]: Wąwóz Gorczyński [DV57], 13 VI 1973, 1 ex., leg. RB [MIZ]; Facimiech [DV57], 28 VI 1973, 1 ex.. leg. BB [MIZ]; Flaki [DV57]. 4 VIII 1997. 1 ex., leg. BB [MIZ].

A widespread European species, reaching Western Siberia. Often captured in Poland, which is confirmed by numerous specimens deposited in the reviewed collections. In the present study, the authors follow the interpretation that Isomira semiflava (KÛsTER, 1852) is a younger synonym of I. murina murina (regarded separate species in KFP). The species inhabits lowlands and forelands in all Poland. The ISEA collection contains specimens from habitats in three KFP regions (Eastern Sudety Mts., Sandomierska Lowland, Podlasie Lowland) not listed in KFP. The MIZ collection includes numerous voucher specimens for faunistic records of B. Burakowski*. In 1982, Dubrovina described a subspecies of $I$. murina ciscaucasica occurring in the western part of Siberia and in Far East. For this reason, a nominotypical subspecies of Isomira mirina murina occurs in Poland. According to earlier interpretation that I. semiflava is a separate species, there were reasons for placing it at the Polish Red List, DD category (PAwlowski et al. 2002). Presently, there is no reason to treat $I$. murina murina (an older synonym of $I$. semiflava) as an endangered species and keep it in Polish Red List. In ISEA collection, there is also a specimen (det. E. WEISE 1971) on the basis of which Isomira icteropa (KÜSTER, 1852) was reported from Poland by Szymczakowski (1973), which was later repeated in KFP. In this group of species, precise identification of species is possible only for male specimens (DUBROVINA 1982), while the discussed specimen is a female, identical as collected female specimens of $I$. murina. Bearing in mind the above, we have to state that I. icteropa has not yet been found in Poland, and I. murina is the only representative of its genus in our country.

\section{Mycetocharis axillaris (PAYKULL, 1799)}

Pomeranian Lake District: Szczecin (Stettin) [VV62], date missing, 2 exx., coll. MIZ.

Masurian Lake District: Krosno [DE49] ad Orneta, 4 IX 1980, 1 ex.. leg. BB [MIZ]; Kulik [EE43] ad Pisz, 22 X 1962, 5 exx., leg. BB [MIZ].

Wielkopolska-Kujawy Lowland: Rogalin [XT38], 23 III 1954, 11 exx., leg. BB [MIZ]; Karszew [CC57] ad Dąbie. 1 VI 2004, 2 exx., leg. MK [ISEA]; Toruń [CD37]. city park. 20 IX 1956, 2 exx., leg. BB [MIZ]; Toruń: Kępa Bazarowa [CD37], 20 IX 1956, 6 exx., leg. BB [MIZ].

Mazowiecka Lowland: Skierniewice [DC45]. 25 VI 1895, 2 exx.. leg. SS [ISEA]; Ruda [DC45] ad Skierniewice, 8 V 1955, 13 exx., leg. BB [MIZ]; Puszcza Kampinoska Forest: Nartowa Góra [DC69]. 14 V 1964, 4 exx., leg. BB [MIZ]; Puszcza Kampinoska Forest: Roztoka [DC79]. 5 V 1963, 2 exx.. leg. BB [MIZ]: Rózin [DC79]. 8 I 1961. 3 exx.. leg. BB [MIZ]: Debly [DC79]. 23 X 1960 and 8 I 1961, 9 exx., leg. BB [MIZ]: Warszawa-Bielany [DC99]. 24 VI 1894, 20 XI 1895 and 1-5 VI 1897, 7 exx.. leg. WM [USMB], 13 V 1950, 28 III 1954, 24 IV and 27 XI 1955. 22 I and 5 IV 1956, 22 XII 1957. 16 II and 7 IV 1958, 4 II and 14 V 1962. 15 III 1963, 7 V 1966, 105 exx., leg. BB [MIZ]; Warszawa-Mlociny [DC99]. 28 XI 1953, 11 XII 1955. 6 I 1958, 25 X 1959, 21 III 1969, 29 exx., leg. BB [MIZ]; Grochale Stare [DD70], 20 II 1972. 
2 ex.. leg. BB [MIZ]; Glusk [DD70], 6 XI 1960, 3 exx.. leg. BB [MIZ]: Pomiechówek [DD71]. 15 IV 1956 and 19 IV 1958, 5 exx.. leg. BB [MIZ]; Palmiry [DD80], 20 III 1960, 3 exx.., leg. BB [MIZ]; Warszawa-Morysinek [EC07]. 17 IV and 20 V 1955, 4 exx., leg. BB [MIZ]; Warszawa: Ogród Saski [EC08]. 7 IV 1977. 2 exx., leg. BB [MIZ]; Warszawa-Agrykola [EC08]. 10 III 1959, 13 exx., leg. BB [MIZ]; Świder [EC17], 15 VII 1900. 1 ex., leg. WM [USMB]; Brzóza [EC21] ad Kozienice. 7 VII 1956, 3 exx.. leg. BB [MIZ]: Gliniak [EC37]. 17 III 1923. 3 exx.. leg. TE [MIZ]; Kacice [ED03] ad Pultusk. 24 III 1955, 1 ex., leg. BB [MIZ]; Porządzie [ED23] ad Wyszków. 7 X 1962. 7 exx., leg. BB [MIZ].

Bialowieża Primeval Forest: Kosy Most [FD95], f. comp. 130D, 16 VI 1988, 1 ex.. leg. DK [ISEA].

Lower Silesia: Sobótka [XS24], 3 IV 1955, 1 ex., leg. BB [MIZ]: Henryków [XS41], 18 VI 1970, 2 exx.. leg. BB [MIZ].

Upper Silesia: Katowice-Murcki [CA56], 30 III 1962, 2 exx., leg. BB [MIZ]; Koszęcin [CB41], 4 VII 1989, 1 ex., leg. HS [ISEA].

Kraków-Wieluń Upland: Zloty Potok [CB81]. 9 V 1961. 13 exx.. leg. BB [MIZ]; Siedlec [CB81] ad Zloty Potok. 7 XI 1952, 5 exx., leg. BB [MIZ]; Kraków-Panieńskie Skaly [DA14]. 1 VII 1917, 1 ex., leg. EM [MIZ]; Kraków-Wola Justowska [DA14]. 27 VI 1917, 1 ex.., leg. SS [ISEA]: Kraków-Łobzów [DA24]. 2 VI 1900. 5 exx.. leg. MR [ISEA]: Kraków [DA24]. 29 Listopada Ave., 8 VI 1990, 1 ex., leg. DK [ISEA].

Malopolska Upland: Krzyżanowice [DA68], 9 VII 1953, 2 exx., leg. BB [MIZ]; BuskoZdrój [DA89]. 1 VII 1956. 1 VII 1958. 5 exx.. leg. BB [MIZ].

Lubelska Upland: Macoszyn [FB79]. 15 VI 1994, 2 exx., leg. MA [ISEA].

Roztocze Upland: Zwierzyniec [FB30], 11 VIII 1911, 1 ex., leg. FF, 11 VI 1955, 16 exx., leg. BB [MIZ].

Western Beskidy Mts.: Wisla-Czarne [CA40]. 1 ex., leg. BB [MIZ]; Wielka Czantoria Mt. [CA40], 19 VI 1962, 8 exx., leg. BB [MIZ]; Zwardoń [CV58], 18 III 1956, 5 exx., leg. BB [MIZ]; Barania Góra Mt. [CV59], 5 VII 1955. 2 exx., leg. BB [MIZ].

Eastern Beskidy Mts.: Tylawa [EV57]. 7 X 1961. 3 exx.. leg. BB [MIZ]: Holubla [FA11]. 29 VI 1884 and VI 1886, 2 exx.. leg. BK [ISEA]; Olszany [FA11] ad Przemyśl, 24 IX 1962. 1 ex., leg. BB [MIZ]: Przemyśl-Prałkowce [FA21]. 25 V 1958, 1 ex.. leg. BB [MIZ].

Bieszczady Mts.: Ustrzyki Górne [FV14]. 6 VI 1956, 6 VI 1958, 27 IX 1962, 14 VII 1969. 33 exx., leg. BB [MIZ].

A species distributed in central and south-eastern part of Europe. According to KFP, the beetles are collected in few scattered localities, but in most of KFP regions (except of mountains and eastern parts of the country), which is supported from not numerous but recent faunistic reports (Pawıowski et al. 2000; Konwerski \& Przewoźny 2001; Kubisz \& Tsinkevich 2001; Gosik 2007; Bunalski et al. 2010). In ISEA collection, there are specimens captured in Lubelska Upland (no localities from that region have been listed in KFP). MIZ collection includes numerous voucher specimens for notes signed "B. Burakowski*" (apart from localities from Baltic Coast).

\section{Mycetocharis flavipes (FABRICIUS, 1792)}

Masurian Lake District: Krosno [DE49] ad Orneta. 19 IX 1977. 4 IX 1980, 8 IV 1983, 11 exx., leg. BB [MIZ]; Kulik [EE43] ad Pisz. 22 X 1962. 1 ex., leg. BB [MIZ]; Puszcza Romincka 
Forest: ad Pluszkiejmy [EF91], 30 VI 1987, 2 exx.. leg. JG [ISEA]; Porzecznik [FE27] ad Augustów, 3 IV 1955. 1 ex., leg. BB [MIZ]; Augustów [FE36], 31 III 1955. 1 ex.. leg. BB [MIZ].

Wielkopolska-Kujawy Lowland: Nowa Sól (Neusalz) [WT43], date missing. 1 ex., coll. MIZ.

Mazowiecka Lowland: Żyrardów [DC66], 16 XII 1951. 12 exx.. leg. BB [MIZ]; Zaborów [DC79]. 21 III 1965. 20 exx., leg. BB [MIZ]; Puszcza Kampinoska Forest: Rózin [DC79]. 8 I 1961, 1 ex., leg. BB [MIZ]; Puszcza Kampinoska Forest: Ławy [DC79]. 13 IX 1960, 1 ex., leg. BB [MIZ]; Puszcza Kampinoska Forest: Grabowy Grunt [DC79], 28 XII 1953, 8 exx.., leg. BB [MIZ]; Puszcza Kampinoska Forest: Wywrotnia Góra [DC89]. 5 I 1958, 19 exx.. leg. BB [MIZ]; Puszcza Kampinoska Forest: Sieraków [DC89], 6 III 1955, 2 III and 21 IX 1958, 8 XI 1959, 18 IX 1960, 40 exx., leg. BB [MIZ]; Warszawa: Skierniewicka str. [DC98], 12 VI 1953, 4 exx., leg. BB [MIZ]: Dziekanów Leśny [DC99]. 11 XI 1956. 5 IV 1959. 21 V 1961. 4 exx.. leg. BB [MIZ]: Łomianki [DC99], 18 I and 4 IV 1953, 10 V 1955. 6 V 1956, 21 exx., leg. BB [MIZ]: WarszawaBuchnik [DC99], 28 III 1957, 1 ex., leg. BB [MIZ]; Warszawa-Mlociny [DC99], 10 II 1952, 6 I and 21 III 1958, 21 III 1968, 9 exx., leg. BB [MIZ]: Warszawa-Bielany [DC99]. 23 VI 1893, 14 VI 1895, 30 VII 1897, 27 VI 1898, 4 exx., leg. WM [USMB], 26 VI 1935, 1 ex., leg. AB, 17 VI 1952, 24 IV and 27 XI 1955, 5 IV 1956, 16 V 1958, 14 II 1959, 19 exx., leg. BB, 20 VI 1978. 1 ex.. leg. AJ [MIZ]: Glusk [DD70]. 6 XI 1960, 1 ex.. leg. BB [MIZ]: Izabelin [DD99]. 3 III 1957, 9 exx., leg. BB [MIZ]; Warszawa-Natolin [EC07], 6 VI 1888, 9 V 1892, 3 exx., leg. WM [USMB]; Warszawa-Morysinek [EC07], 11 IV 1955 and 7 I 1962, 22 exx., leg. BB [MIZ]: Warszawa: ul. Frascati [EC08]. 21 VII 1955, 1 ex.. leg. PD [MIZ]: Świder [EC17]. 25 VI 1932. 4 exx.. leg. BB [MIZ]; Świdry Male [EC17]. 16 VI 1964 and 23 V 1965. 2 exx.. leg. BB [MIZ]: Otwock [EC17], 4 VI 1894, 1 ex., leg. WM [USMB]; Warszawa-Miedzeszyn [EC18], 11 VI 1956, 1 ex.. leg. BB [MIZ]; Maciejowice [EC31] ad Kozienice, 7 VII 1956, 2 exx., leg. BB [MIZ]: Gliniak [EC37]. 17 VI 1923, 1 ex., leg. TE [MIZ]: Siennica [EC47], 26 V 1932. 2 exx.. leg. AB [MIZ]; Rudka [EC57] ad Mińsk Mazowiecki, 12 I 1958, 5 exx., leg. BB [MIZ]; Klembów [ED20], 20 IV and 13 V 1958, 27 III 1960. 12 XI 1962, 13 exx., leg. BB [MIZ]: Małkinia [ED63]. IX 1952. 1 ex.. leg. BB [MIZ]; ,Warszawa”, 23 VI 1901. 1 ex., leg. WM [USMB].

Bialowieża Primeval Forest: ad Podcerkwa [FD83]. 22 VI 1995, 1 ex., leg. JG [ISEA]; f. comp. 579D [FD83]. 25 VI 1991. 1 ex., leg. DK [ISEA]; f. comp. 312A/313B [FD84], 2 VIII 1990, 1 ex., leg. JG [ISEA]; f. comp. 450 [FD84]. 19 XI 1959, 1 ex.. leg. BB [MIZ]; f. comp. 80D [FD85]. 26 VI 1990, 1 ex.., leg. DK [ISEA]; f. comp. 399A [FD94], 23 V 1993, 1 ex.. leg. JL [USMB]; f. comp. 192 [FD95], 2 IV 1966, 1 ex., leg. BB [MIZ].

Upper Silesia: Tarnowskie Góry-Pniowiec (Pniow) [CA49], 1 VI 1934, 1 ex., leg. KI [USMB].

Malopolska Upland: Krzyżanowice [DA68], 21 V 1955, 2 exx.. leg. BB [MIZ]; BuskoZdrój [DA89]. 19 V 1952 and 1 VII 1956, 9 exx.. leg. BB [MIZ].

Świętokrzyskie Mts.: Kielce [DB73], 1908, 1 ex.., leg. TE [MIZ]; Św. Katarzyna [DB84]. 20 VI 1954, 1 ex., leg. PD [MIZ].

Lubelska Upland: Nowiny [FB77] ad Chelm Lubelski. 8 VI 1991, 1 ex.. leg. ŁP [ISEA]; Macoszyn [FB79], 15 VI 1994, 9 exx., leg. MA [ISEA].

Sandomierska Lowland: Horyniec [FA66], 1-16 V 1888, 1 ex.., leg. SS [ISEA].

Western Beskidy Mts.: Wisla [CA40]. 24 VI 1955 and 13 VI 1959. 3 exx., leg. BB [MIZ]: Polichty [DA91] ad Gromnik, 22 V 1999, 1 ex.. leg. LB [ISEA]; Rytro [DV78]. 2 VI 1897, 1 ex.. leg. SS [ISEA]; Nowy Sacz [DV79]. 18 VI 1892, 1 ex., leg. SS [ISEA].

Eastern Beskidy Mts.: Radoszyce [EV76] ad Nowy Łupków. 28-30 V 1882. 2 exx.. leg. BK [ISEA]; Góry Slonne: Wujskie [EV98]. 17 VI 1954. 1 ex., leg. AR [MIZ]; Góra Biała (Splawa) 
[FA10], 7 VI 1884, 1 ex., leg. BK [ISEA]; Olszany [FA11] ad Przemyśl, 24 IX 1962, 1 ex., leg. BB [MIZ]; Holubla [FA11], 25 V-15 VI 1884 and VI 1886, 4 exx., leg. BK [ISEA]: PrzemyślPralkowce [FA21]. 25 V 1956 and 25 V 1958, 4 exx., leg. BB [MIZ]; Przemyśl-Ostrów [FA21]. $31 \mathrm{~V} 1879$ and VI-VII 1880, 2 exx., leg. BK [ISEA].

Bieszczady Mts.: Ustrzyki Górne [FV23]. 13 V 1968, 1 ex., leg. BB [MIZ]; Tarnica Mt. [FV23]. 24 VI 1953. 1 ex.. leg. BB [MIZ].

The species occurs in the northern and central part of Europe, southern border of its range reaching northern Italy and Romania. According to KFP, it is rarely and sporadically found in Poland, and most of data on its distribution is based on findings from last century. This applies to some of the KFP regions (Pomeranian Lake District, Upper Silesia, Lower Silesia, Trzebnickie Hills, Sandomierska Lowland) and is still true. The studied collections contain also numerous specimens captured in the second part of $20^{\text {th }}$ century (including voucher specimens for the notes signed „B. BURAKOWsKI" "), which is also confirmed by data published in the last decade (KuBISz et al. 2000; PAwlowski et al. 2000; Kubisz \& Tsinkevich 2001; Gutowski et al. 2006; Bunalski et al. 2010). In the ISEA collection, there is one specimen caught in 1888 in Sandomierska Lowland (Horyniec), a region not listed in KFP.

\section{Mycetocharis humeralis (FABRICIUS, 1787)}

Pomeranian Lake District: Szczecin (Stettin) [VV62]. date missing. 3 exx., coll. MIZ; Puszcza Bukowa: Radziszewko [VV70], 15 VII 1991, ad lucem. 2 exx.. leg. MA [ISEA]; Goleniów (Gollnow) [VV83], date missing, 2 exx., leg. LD [MIZ]; Koszalin (Coeslin) [WA70]. date missing. 4 exx.. leg. AL [MIZ].

Bialowieża Primeval Forest: Bialowieża [FD94]. VII 1985, 1 ex.. leg. JG [ISEA]; f. comp. 130D [FD95], 16 VI 1988, 1 ex.. leg. DK [ISEA].

Upper Silesia: Racibórz (Ratibor) [CA05]. 13 VI 1911, 1 ex.. coll. USMB.

Kraków-Wieluń Upland: Czerna [DA05] ad Krzeszowice, 16 VII 1903, 1 ex., leg. SS [ISEA]: Zabierzów [DA15] ad Kraków, 10 VI 1934, 1 ex.. leg. EM [MIZ]; Kraków [DA24]. near Wawel castle. 29 X 1958, 19 exx.. leg. BB [MIZ]; Kraków: Botanical Garden [DA24], 14 VI 1907. VI 1914, 20 V-16 VII 1937, 5 exx., leg. SP [ISEA].

Lubelska Upland: Kazimierz Dolny [EB68], 6-19 VII 1951, 1 ex., leg. BP [MIZ].

Sandomierska Lowland: Horyniec [FA66]. 23-25 V 1888, 1 ex.. leg. SS [ISEA].

Western Beskidy Mts.: Krzęcin [DA13] ad Skawina, 7 X 1917, 3 exx.. leg. EM [MIZ]; Rytro [DV78], date missing. 1 ex., leg. SS [ISEA].

Eastern Beskidy Mts.: Lipowica [EV48] ad Dukla. 8 VI 1966. 1 ex.. leg. AS [ISEA].

Bieszczady Mts.: Otryt Mt. [FV15], 16 VI 1958, 1 ex., leg. BB [MIZ]; Ustrzyki Górne [FV23], 6 VI 1958, 27 IX 1962, 3 IV and 15 XI 1968, 5 exx.., leg. BB [MIZ]; Tarnica Mt. [FV23]. 24 VI 1953. 1 ex.. leg. BB [MIZ]; Szeroki Wierch Mt. [FV23], 9 VI 1958, 1 ex., leg. BB [MIZ]: Bereżki [FV24], 9 IX 1967, 1 ex., leg. BB [MIZ]: Smolnik nad Sanem [FV25], 14-17 VI 1958. 10 exx., leg. BB [MIZ].

A widespread species in all Europe, but rarely found in all places. According to KFP, reported from few localities in Poland in only eight regions, among which Upper Silesia has not been listed. However, faunistic data coming from reviewed collections and 
publication (Kubisz \& Szwalko 1991; Pawlowski et al. 2000; Byk \& Byk 2001; Kubisz \& Tsinkevich 2001; Bunalski et al. 2010) prove that the species probably inhabits all Poland, with the exception of high mountain areas.

\section{Mycetocharis maura (FABRICIUS, 1792) [=linearis (ILLIGER, 1794)]}

Pomeranian Lake District: Bielinek nad Odrą [VU46], 22 VI 1993, 1 ex., leg. DK [ISEA]; Szczecin (Stettin) [VV62], date missing. 9 exx.. coll. MIZ; Koszalin (Coeslin) [WA70], date missing. 2 exx.. leg. AL [MIZ]; Krzeszna [CF01] ad Kartuzy, 15 IV 1955, 8 exx.. leg. BB [MIZ].

Wielkopolska-Kujawy Lowland: Nowa Sól (Neusalz) [WT43], 22 V-20 VI 1905, 16 exx.. leg. Schreiber. 30 III 1957, 2 exx.. leg. BB [MIZ]: Otyń [WT44]. 22 IV 1971. 1 ex.. leg. BB [MIZ]; Bobrowniki [WT54], 23 IV 1971, 1 ex., leg. BB [MIZ]; Smolarnia [WU97]. 19 III 1954. 2 exx.. leg. BB [MIZ]: Rogalin [XT38]. 17 VI 1975, 1 ex.. leg. MB [ISEA]: Poznań [XU21], 18 IX 1955. 1 ex., leg. BB [MIZ]: Kolo [CC38], 31 XII 1999. 1 ex.. leg. MK [ISEA]: Kazimierz Biskupi [CD00] ad Shupca, 16 VI 1907. 1 ex.. leg. HL [USMB].

Mazowiecka Lowland: Warszawa-Bielany [DC99]. 23 I 1953, 27 XI 1955, 5 IV 1956. 26 XII 1982. 19 exx.. leg. BB [MIZ]: Stary Otwock [EC17]. 13 VI 1928. 1 ex., leg. TE [MIZ]: Brzóza [EC21] ad Kozienice, 7 VII 1956, 1 ex., leg. BB [MIZ].

Lower Silesia: Świebodzice [WS93]. 6 VI 1959 and 16 IV 1960. 2 exx.., leg. BB [MIZ]; Ligota Wlk. (Ellguth Ottmachau) [XR49] ad Otmuchów. VI 1910 and VI 1919, 2 exx., leg. ED [USMB]; Wroclaw-Park Szczytnicki [XS46]. 30 IV and 3 V 1961, 3 V 1963, 112 exx., leg. BB [MIZ].

Upper Silesia: Chelmek [CA75], 15 V 1898. 11 exx.. leg. SS [ISEA]. 15 V 1907, 2 exx., leg. HL [USMB]: Zawadzkie [CB20]. 20 II 1928, 1 ex.. leg. KI [USMB].

Kraków-Wieluń Upland: Ojców [DA16]. Góra Chełmowa, 9 VII 1964. 1 ex., leg. JP [ISEA].

Sandomierska Lowland: Klaj [DA53]. 11 V 1897 and 24 V 1901. 2 exx.. leg. SS [ISEA].

Western Sudety Mts.: Maciejowiec [WS44] ad Lwówek Śląski, 25 XI 1960, 8 IX 1964. 26 IV 1971, 5 exx.., leg, BB [MIZ].

Western Beskidy Mts.: Wielka Czantoria Mt. [CA40]. 13 VII 1962. 1 ex.. leg. BB [MIZ]; Kubalonka pass [CV49]. 18 VI 1959, 1 ex.. leg. BB [MIZ]; Glogoczów [DA23], 20-29 V 1897. 3 exx.. leg. SS [ISEA].

Eastern Beskidy Mts.: Radoszyce [EV76] ad Nowy Łupków. 28-30 V 1882, 1 ex.. leg. BK [ISEA]: Panieński Czub [FA00] ad Bircza, 22 V 1879, 1 ex.. leg. BK [ISEA]; Holubla [FA11]. 29 VI 1884 and VI 1886, 4 exx.. leg. BK [ISEA]: Krasiczyn [FA11]. 22 IX 1962, 2 exx.., leg. BB [MIZ].

A widespread species in Europe. Reported from virtually all KFP regions except for the eastern part of the country. This beetle inhabits lowlands and lower mountain habitats. It probably occurs in all the country, but is rarely captured and there are few faunistic data published (ByK \& ByK 2004; Bunalski et al. 2010). The MIZ collection includes most of voucher specimens for notes signed B. Burakowski* (the specimen from Sopot is missing).

\section{Mycetocharis pygmaea (REDTENBACHER, 1874)}

Sandomierska Lowland: Klaj [DA53], 25 V 1904, 1 ex., leg. MR [ISEA]. 
A species inhabiting south-eastern Europe and southern part of the central Europe, very rarely and sporadically found. According to KFP, it is known only from four regions in southern Poland. This information comes from publications from the first half of the XX century. In MIZ collection, there are no voucher specimens for faunistic reports (B. Burakowski*, Burakowski 1976) from Upper Silesia and Sandomierska Lowland (vicinity of Jaroslaw), while the ISEA collection includes one specimen captured in 1904 (Klaj, Puszcza Niepolomicka Forest). This species should probably be placed in the Polish Red List, category DD or EX.

\section{Prionychus ater (FABRICIUS, 1775)}

Baltic Coast: Slawoszyno [CF17] ad Wejherowo, 24 IX 1955, 2 exx., leg. BB [MIZ]; Rzucewo [CF36] ad Puck. 20 V 1938, 1 ex., leg. AB [MIZ]: Jastarnia [CF46], 10 VIII 1957. 1 ex.. coll. MIZ, 11 VIII 1991, ad lucem. 1 ex.. leg. MA [ISEA]: Tolkmicko [DF02]. 20 VII 1957. 1 ex., leg. RW [MIZ].

Pomeranian Lake District: Szczecin (Stettin) [VV62]. date missing. 2 exx., coll. MIZ; Koszalin [WA70], 3 IV 1970, 1 ex., leg. BB [MIZ]: Plutowo [CE21] ad Chelmno. 20 VIII 1965. 1 ex., leg. WS [ISEA].

Wielkopolska-Kujawy Lowland: Nowa Sól (Neusalz) [WT43], 6 VI 1901, 4 VIII 1907. 4 IV and 5 V 1908, 4 exx., coll. MIZ; Czempiń [XT27], 13 VII 1997. 1 ex.. leg. WJ [ISEA]: Kościelec [CC38] ad Kolo, 15 II 1998, 2 exx., leg. MK [ISEA]; Łęczyca [CC76], 20 V 1938 and 12 V 1939, 2 exx., leg. EM [MIZ].

Mazowiecka Lowland: Wola Pękoszewska [DC65]. 3 V 1977. 2 exx.. leg. BB [MIZ]; Józefów [DC69]. 28 VI 1931, 1 ex.. leg. BB [MIZ]; Milanówek [DC77]. 2 VII 1957, 1 ex.. leg. SY [MIZ]; Lasy Mlochowskie Forest [DC86], 5 VI 1949, 1 ex., leg. AR [MIZ]: Żbików [DC88]. 5 X 1958, 1 ex.. leg. BB [MIZ]: Dziekanów Leśny [DC99]. 6 I 1955. 13 exx.. leg. BB [MIZ]: Warszawa-Buchnik [DC99], 19 VII 1956, 1 ex., leg. BB [MIZ]; Warszawa-Mlociny [DC99]. 23 XII 1951, 23 XII 1953, 10 IV and 11 XII 1955, 7 exx., leg. BB [MIZ]: Warszawa-Bielany [DC99]. 17 VI 1889, 1 ex.. leg. WM [USMB]. 5 VII 1950. 1 VII 1954. 30 I and 24 IV 1955. 18-20 II 1959. 7 II 1960, 4 IV 1961, 13 III and 11 XII 1964, 27 II 1966, 12 V 1969, 25 exx.. leg. BB [MIZ]; Jabłonna [DD90], 20 VIII 1908, 1 ex.. leg. TE [MIZ]; Warszawa-Morysinek [EC07]. 5 XII 1954. 8 VI 1956. 27 IV 1964. 5 exx.. leg. BB [MIZ]: Warszawa-Agrykola [EC08]. 2 III 1959, 2 exx., leg. BB [MIZ]: Warszawa-Saska Kępa [EC08]. 2 VIII 1898, 1 ex., leg. WM [USMB], 25 VI 1953, 1 ex., leg. MZ, 9 V 1955, 2 exx.. leg. BB [MIZ]; Warszawa-Ogród Saski [EC08]. 7 IV 1977. 1 ex.. leg. BB [MIZ]: Świder [EC17]. 4 V 1910. 1 ex.. leg. TE. 15 VI 1948. 1 ex., leg. PD [MIZ]; Otwock Stary [EC17], 20 VI 1947, 1 ex., leg. ZW [MIZ]; WarszawaWygoda [EC18], 18 VIII 1960, 1 ex., leg. WD [MIZ]; Serock [ED01], 4 III 1961, 2 exx., leg. BB [MIZ]: Klembów [ED20]. 31 X 1953 and 13 V 1958, 2 exx.. leg. BB [MIZ]: Sulejów [ED31]. 3 VI 1898, 1 ex., leg. WM [USMB]; Kalinowo [ED65], 8 VIII 1935. 1 ex., coll. MIZ; „Warszawa”. 16 VI 1948, 1 ex., leg. JF [MIZ].

Bialowieża Primeval Forest: Białowieski N. P., f. comp. 340 [FD94], 13 VI 1965, 5 exx.. leg. BB [MIZ]; f. comp. 400 [FD94]. 25 V 1974, 1 ex.. leg. BB [MIZ].

Lower Silesia: Ligota Wielka (Ellguth Ottmachau) [XR49] ad Otmuchów. VII 1910. 1 ex., leg. ED [USMB]: Ząbkowice Śląskie [XS20], 27 VI 1948, 1 ex.. leg. KL [MIZ]: Sobótka [XS24]. 2 IV 1956, 3 exx., leg. BB [MIZ]: Wroclaw-Swojczyce [XS46]. 24 III 1954, 2 exx., leg. BB [MIZ]; Starościn (Sterzendorf) [XS95] ad Namyslów, 10 III 1927, 4 exx.. leg. KI [USMB]. 
Upper Silesia: Pokój (Carlsruhe O/S) [XS94], 12 IV 1927, 2 exx., leg. HN [USMB]: Rybnik-Paruszowiec (Paruschowitz) [CA25]. 12 VII 1910. 1 ex.. leg. SC [USMB]; KatowiceMurcki [CA56]. 30 III 1962, 1 ex.. leg. BB [MIZ]: Bytom (Beuthen) [CA58], 14 VII 1928. 1 ex.. leg. KI. 7 V 1934, 1 ex., leg. HN [USMB]: Chelmek [CA75]. 5 VII-14 VIII 1878, 2 exx.. leg. SS [ISEA].

Kraków-Wieluń Upland: Kraków-Wola Justowska [DAl4], 2 V and 7 VII 1878, 3 exx.. leg. SS \& MR [ISEA]: Kraków: Botanical Garden [DA24], 15-18 VI 1937, 3 exx.. leg. SP [ISEA]; Kraków-Mydlniki [DA24], 18 VII 1927, 1 ex., leg. SP [ISEA]; Koniecpol [DB02], 19 VII 1983. 1 ex.. leg. TW [ISEA].

Malopolska Upland: Michałów [DA69] ad Pińczów, 15 V 1954, 1 ex., leg. BB [MIZ]; Radom [EB19]. 24 VI 1897, 2 exx., leg. SS [ISEA].

Lubelska Upland: Kazimierz Dolny [EB68], 31 VII 1907 and V 1934. 2 exx., leg. TE [MIZ]: Bochotnica [EB69]. 28 V 1955. 1 ex.. leg. BB [MIZ]: Pulawy [EB69]. 9 VII 1950. 5 exx., leg. BB [MIZ].

Roztocze Upland: Florianka [FB30]. date missing. 1 ex.. leg. FF [MIZ]; Zwierzyniec [FB30]. Bukowa Góra. 7-8 VI 1955, 2 exx., leg. BB [MIZ].

Sandomierska Lowland: Wolica [EA24] ad Dębica. 17 III 2006, 1 ex.. leg. AT [ISEA]; Leżajsk [FA06]. 20 IX 1957, 2 exx., leg. BB [MIZ]; Horyniec [FA66]. 1-31 V 1889, 1 ex.. leg. SS [ISEA].

Western Beskidy Mts.: Kraków-Wróblowice [DA23]. 21 VII 1998. 1 ex.. leg. DK [ISEA]; Stadniki [DA42] ad Gdów, 15 VII 1987. 1 ex.. leg. BW [ISEA]: Lapanów [DA42]. 30 VII 1916 and 23 VI 1917, 2 exx., leg. EM [MIZ]; Zborczyce [DA43], 29 VIII 1895, 1 ex.. leg. SS [ISEA]: Rabka [DV29]. VII-VIII 1879, 1 ex., leg. SS [ISEA].

A species widespread everywhere in Europe. Common in Poland, which is supported by numerous reports on its occurrence in publications (KuBIsz et al. 2000; KuBISZ \& Tsinkevich 2001; Konwerski \& Przewó́ny 2001; Byk \& Byk 2004; Klasiński 2008 ). Juvenile stages inhabit rotting broadleaved trees, sometimes coniferous trees; mature beetles show nocturnal activity pattern and secretive nature. In KFP, it is reported from virtually all regions; voucher specimens for notes of B. BuRAKOwski* are deposited in MIZ collection.

\section{Prionychus melanarius (GERMAR, 1813)}

Pomeranian Lake District: Szczecin (Stettin) [VV62]. date missing. 3 exx., coll. MIZ; Korzybie [XA21] ad Slawno, 31 VII 1954, 1 ex., leg. BB [MIZ]: Tleń [CE14], 12 VIII 1922. 1 ex., leg. TE [MIZ].

Masurian Lake District: Augustów [FE36], 15 XII 1954, 6 exx.. leg. BB [MIZ].

Wielkopolska-Kujawy Lowland: Bobrowniki [WT54], 23 IV 1971, 1 ex., leg. BB [MIZ].

Mazowiecka Lowland: Łomianki [DC99], 18 XII 1955, 6 V 1956, 5 exx., leg. BB [MIZ]; Warszawa-Bielany [DC99]. 2 V 1890, 1 ex.. leg. WM [USMB]. 13 III 1964. 1 ex.. leg. BB [MIZ]; Otwock [EC17], 4 VI 1894 and 26 VII 1899. 2 exx., leg. WM [USMB]: Świder [EC17]. 10-22 VII 1900, 2 exx., leg. WM [USMB]; Brzóza [EC21] ad Kozienice. 7 VII 1956, 14 exx., leg. BB [MIZ]: Celestynów [EC26]. 16 X 1955, 1 ex.. leg. BB [MIZ].

Bialowieża Primeval Forest: f. comp. 499 [FD94], 30 VII 1955, 4 exx.. 1eg. BB [MIZ].

Lower Silesia: f. div. Popielów [XS93]. 30 VI 1951, 2 exx., leg. ZC [MIZ]. 
Upper Silesia: Katowice-Murcki (Emmanuelssegen) [CA56], 17 VI 1923, 5 exx., coll. USMB, 16 XI 1954, 2 exx.. leg. BB [MIZ].

Roztocze Upland: Zwierzyniec [FB30], 3 VII 1910, 1 ex., leg. TE [MIZ]; Panasówka [FB30]. 7 VII 1911. 1 ex.. leg. TE [MIZ]: Florianka [FB30], date missing. 1 ex.. leg. FF [MIZ]: Kaczorki [FB40]. 4 VI 1961, 1 ex., leg. PD [MIZ]: Nart [FB50], 14 VII 1910, 1 ex., 1eg. TE, 20 VII 1912, 1 ex.., leg. FF [MIZ].

Sandomierska Lowland: Nisko [EA89], 26 VII 1956. 2 exx., leg. WB \& EK [MIZ]; Zaleszany [EB61] ad Sandomierz, 12 V 1891, 1 ex., leg. SS [ISEA].

A rare species in Poland. According to KFP, it has been reported from few scattered locations in various parts of the country ( 9 KFP regions); MIZ collection includes specimens with localities given as B. BuRAKOwsKI* note (except for Lubelska Upland: Kaczorki). The occurrence of this species in Poland has been supported several times in the last decade, in the following publications: KuBisz et al. 2000, GuTowski et al. 2006, Gutowski et al. 2010, Kubisz \& Tsinkevich 2001. Larvae live in hollows at the bottom of tree trunks, under tree bark, in stumps and logs, mainly of broadleaved trees.

\section{Pseudocistela ceramboides ceramboides (LinnaEUs, 1761)}

Baltic Coast: Slowiński N. P., Wydma Czolpińska [XA46]. 11-20 VII 1991, 2 exx.. leg. MA [ISEA]: Białogóra [XA98], 10 VII 1991, 1 ex.. leg. MA [ISEA]; Jastarnia [CF46], 9 VII 1991. 1 ex., leg. MA [ISEA].

Pomeranian Lake District: Bielinek nad Odrą nat. res. [VU46]. 1 VI 1983, 1 ex., leg. BB [MIZ]: Szczecin (Stettin) [VV62]. 10 II 1912, 2 exx., leg. GS [MIZ]; Miastko [XV28], 3 VI 1982, 1 ex., leg. JM [ISEA]: Tleń [CE14]. 30 VII 1922. 1 ex.. leg. TE [MIZ].

Masu rian Lake District: Stare Jabłonki [DE35], 19 VI-13 VII 2004, 2 exx., leg. EN [ISEA]; Szeroki Bór [EE44] ad Pisz. 22 X 1962. 2 exx.. leg. BB [MIZ]; Porzecznik [FE27] ad Augustów. 3 IV 1955, 2 exx., leg. BB [MIZ]; Puszcza Augustowska Forest: Gruszki [FE67], 9 VII 1990, ad lucem. 2 exx., leg. MA [ISEA]; Puszcza Romincka Forest: f. distr. Boczki [FF02]. 29 III 1955 and 15 X 1957. 11 exx., leg. BB [MIZ]. 12 VI 1990, ad lucem. 1 ex.. leg. MA [ISEA].

Wielkopolska-Kujawy Lowland: Nowa Sól (Neusalz) [WT43]. 7 VI-1 VII 1906, 30 VI 1907. 25 VI 1908. 11 exx. coll. MIZ: Otyń [WT44] ad Nowa Sól. 22 IV 1971. 3 exx.. leg. BB [MIZ]; Osowa Góra [XT07], 29 VI 1994, 1 ex.. leg. MK [ISEA]; Rawicz (Rawitsch) [XT22], IV 1903, 1 ex., leg. FK [MIZ]; Puszczykowo [XT29] ad Poznań, 30 VI 1994, 5 exx. ad lucem. leg. MA [ISEA]: Sycyn Dolny [XU13]. 10 V 1993. 1 ex.. leg. MA [ISEA].

Mazowiecka Lowland: Puszcza Kampinoska Forest: Nartowa Góra [DC69], 22 III 1953. 2 exx.. leg. BB [MIZ]; Puszcza Kampinoska Forest: Debły [DC79]. 8 XI 1953, 1 ex.. leg. BB [MIZ]; Dziekanów Leśny [DC99]. 9 IV 1961. 1 ex.. leg. BB [MIZ]; Warszawa-Buchnik [DC99]. 19 VII 1956 and 1 V 1957. 3 exx., leg. BB [MIZ]: Warszawa-Bielany [DC99]. 16 V 1958, 1 ex.. leg. BB [MIZ]: Palmiry [DD80], 20 III 1960, 3 exx., leg. BB [MIZ]; Jablonna [DD90]. 21 VI 1893, 1 ex., leg. WM [USMB]; Warszawa-Morysinek [EC07], 20 V 1955, 1 ex., leg. BB [MIZ]: Warszawa-Natolin [EC07], 14 VI 1888, 1 ex., leg. WM [USMB]: Świder [EC17], 15 VII 1900. 3 exx. leg. WM [USMB]; Sewerynów [EC21] ad Kozienice, 30 VII 1961. 1 ex.. leg. BB [MIZ]; Dębe Wielkie [EC38]. 5 VII 1918 and 15 VII 1919. 2 exx.. leg. TE [MIZ]: Dąbkowizna [ED10] ad Radzymin. 28-29 VII 1958, 4 exx.. leg. BB [MIZ]: Klembów [ED20]. 21 X 1956. 22 III 1959. 18 XI 1962, 12 exx.. leg. BB [MIZ]; Porządzie [ED23] ad Wyszków, 26 V 1963. 1 ex.. leg. BB 
[MIZ]; Urle [ED41], V 1910, 1 ex., leg. JF [MIZ]; Bojany [ED53] ad Brok, 10 III 1966, 1 ex.. leg. BB [MIZ].

Podlasie Lowland: Puszcza Knyszyńska Forest: Międzyrzecze nat. res. [FE60]. 9 VII 1985. 1 ex. leg. JG [ISEA].

Bialowieża Primeval Forest: f. comp. 637D [FD83], 27 VI 1991, 1 ex., leg. DK [ISEA]; f. comp. 450 [FD84], 19 XI 1959, 1 ex., leg. BB [MIZ]; f. comp. 473 [FD84], 4 VI 1954. 1 ex.. leg. BB [MIZ]: f. comp. 340 [FD94]. 13 VI 1965. 1 ex.. leg. BB [MIZ]: f. comp. 341 [FD94]. 6 VI 1954, 1 ex.. leg. BB [MIZ]; f. comp. 344 [FD94], 11 VIII 1957, 3 exx., leg. BB [MIZ]; f. comp. 399 [FD94]. 31 V 1954. 1 ex.. leg. BB [MIZ]: f. comp. 429 [FD94]. 16 VII 1992. 1 ex.. leg. JG [ISEA]: Białowieża [FD94], 19 II-19 III 1952, 4 exx., leg. BB [MIZ], 9 VIII 1976, 1 ex.. leg. MB [ISEA]; f. comp. 192 [FD95]. 2 IV 1966, 1 ex., leg. BB [MIZ].

Upper Silesia: Katowice-Murcki (Emmanuelssegen) [CA66], 1 IV 1924, 6 VI 1928, 3-9 IV 1929. 18 VI 1936. 7 exx.. leg. KI [USMB].

Kraków-Wieluń Upland: Kraków-Wola Justowska [DA14], 19 V 1879. 1 ex., leg. SS [ISEA]: Ojców [DA16]. 17 VII 1966, 1 ex., leg. JP [ISEA].

Malopolska Upland: Folusz [DA37] ad Miechów. 20 VII 1933, 1 ex.. coll. USMB; Świnia Góra nat. res. ad Bliżyn [DB86]. 19 V 1982. 1 ex.. leg. BB [MIZ]: Radom [EB19]. 24 VI 1897. 1 ex., leg. SS [ISEA].

Świętokrzyskie Mts.: Kielce [DB73]. 14 VI-6 VII 1913, 2 exx.. leg. EI [MIZ]; Łysa Góra Mt. [EB03]. 27 VI 1956, 1 ex., leg. BB [MIZ].

Lubelska Upland: Opole Lubelskie [EB66]. 11 VI 1915. 1 ex., leg. EI [MIZ]; Kazimierz Dolny [EB68]. VI 1935, 1 ex., leg. TE, 28 V 1955, 1 ex., leg. BB [MIZ]; Pulawy [EB69]. VI 1907, 1 ex., leg. TE [MIZ]; Marynopole [EB73] ad Kraśnik. 8 IX 1970, 1 ex., leg. BB [MIZ]: Kosyń [FB79]. 19 V 1994. 1 ex.. leg. MA [ISEA].

Roztocze Upland: Ulów [FA69], 1 VII 1990, ad lucem, 2 exx., leg. MA [ISEA]; Hrebenne [FA87]. 28 VI 1990. 1 ex.. leg. JG [ISEA]: Florianka [FB30]. 20 VI 1912. 2 exx.. leg. FF [MIZ]: Jangi [FB41], 10 VI 1956, 4 exx.. leg. BB [MIZ].

Sandomierska Lowland: Klaj [DA53]. 1904. 1 ex.. leg. MR. 11-23 VII 1935. 2 exx.. leg. SS \& SP [ISEA]; Lubasz [EA07] ad Szczucin, 1943, 1 ex., leg. WN [MIZ]; Nisko [EA89], 18 IX 1957. 2 exx., leg. BB [MIZ]; Zaleszany [EB61] ad Sandomierz, 16 VI 1891, 3 exx., leg. SS [ISEA].

Western Beskidy Mts.: Wisła-Malinka [CA40], 25 VIII 1956, 2 exx., leg. BB [MIZ]; Rytro [DV78], 9 VII 1892, 25 VII 1905, 2 exx., leg. SS [ISEA], 12 VII 1959, 1 ex.. leg. PD [MIZ].

Eastern Beskidy Mts.: Bircza [FA00], $30 \mathrm{~V}$ 1958. 1 ex., leg. BB [MIZ]; Hołubla [FA11]. 31 V 1884, 2 exx., leg. BK [ISEA].

Bieszczady Mts.: Nowy Łupków [EV75]. 29 V 1970, 2 exx., leg. BB [MIZ].

Pieniny Mts.: Krościenko [DV57]. 27 VII 1950. 1 ex.. leg. BB [MIZ].

Poland is inhabited by one of two subspecies - Pseudocistela ceramboides ceramboides; the other subspecies $P$. ceramboides serrata (CHEVROLAT, 1844) is endemic for the Carpathians, reported so far only from Hungary and Slovakia. The nominotypical subspecies is widespread everywhere in Europe. In Poland, the beetle is often met, which is confirmed by a large number of currently published reports (among others, KuBISZ \& Szwalko 1991; Pawlowski et al. 2000; Kubisz et al. 2000; Konwerski \& Przewoźny 2001; Kubisz \& Tsinkevich 2001; Byk \& Byk 2004; Gutowski et al. 2006; Bunalski et al. 2010; Gutowski et al. 2010) and rich museum collections. Its larvae live in rotten 
wood and hollows, mainly in broadleaved trees. KFP does not list reports from Podlasie Lowland, Malopolska Upland and Nowotarska Dale; the ISEA collection contains specimens captured in the first two KFP regions, while the MIZ collection includes voucher specimens for B. BuRaKowski* notes.

\section{Cteniopodini Solier, 1835}

\section{Cteniopus sulphureus (LinNaEus, 1758) [=flavus (Scopoli, 1763)]}

Baltic Coast: Woliński N. P.. Trzciagowo [VV57], 14 VII 1975, 1 ex., leg. PS [ISEA]: Wicko lake [VV67] ad Wolin, 28 VI 1965, 1 ex., leg. AO [MIZ]; Sopot-Kamienny Potok [CF43]. 27 VII 1951. 1 ex.. leg. RB [MIZ]: Tolkmicko [DF02]. 13 VII 1954. 2 exx.. leg. RW [MIZ].

Pomeranian Lake District: Bielinek nad Odrą [VU46], 2 VIII 1985, 18 VII 1987, 8 exx., leg. PS [ISEA]: Krajnik Dolny [VU57]. 19 VII 1957. 1 ex.. leg. EK [MIZ]: Szczecin (Stettin) [VV62], 26 VI 1900, 12 exx., leg. E. Schmidt \& GS [MIZ]; Szczecin-Klucz (Stettin-Klütz) [VV71], 2 VIII 1940, 1 ex., leg. PN [MIZ]; Tleń [CE14], 27-31 VII 1922, 3 exx., leg. TE [MIZ]: Gruczno [CE21]. 5 VII 1995. 1 ex.. leg. MM [ISEA]: Plutowo [CE21] ad Chelmno, 24 VI 1964. 9 exx.. leg. WS [ISEA]; rez. Czarcie Góry ad Świecie [CE32], 10-11 VII 1996, 2 exx.. leg. DK [ISEA]: Nowe Marzy [CE42]. 6 VII 1995, 1 ex., leg. MM [ISEA]: rez. Wiosto Male [CE55] ad Kwidzyn. 12 VII 1996, 1 ex., leg. DK [ISEA].

Masurian Lake District: Orneta [DE49], 9 VII 1952, 3 exx., leg. RB [MIZ].

Wielkopolska-Kujawy Lowland: Owczary [VU71] ad Kostrzyn, 10 VI 2003, 1 ex., leg. DK [ISEA]: Borek [CD18] ad Toruń, 25 VI 1964. 6 exx., leg. WS [ISEA].

Mazowiecka Lowland: Podkowa Leśna [DC87]. 7-19 VII 1934, 51 exx.. leg. TE, 9 VII 1950, 5 exx., leg. WE [MIZ]; Otrębusy [DC87], 25 VII 1956, 9 VII 1961, 22 VII 1962. 20 exx., leg. BB [MIZ]; Zielonki [DC89]. 18 VII 1953, 2 exx., leg. RB [MIZ]; Warszawa-Tarchomin [DC99]. VII 1999, 1 ex. leg. AG [ISEA]: Pomiechówek [DD71], 20 VII 1947, 1 ex., leg. JF [MIZ]; Świder [EC17], 15 VII 1900, 8 exx., leg. WM [USMB]: Dębe Wielkie [EC38], 20 VII 1918, 1 ex.. leg. TE [MIZ]; Zegrze [ED01]. 13 VII 1935, 6 exx., leg. AB, 12-22 VII 1948, 6 exx.. leg. RB [MIZ]; Pultusk [ED04]. 21 VII 1973. 1 ex.. leg. EN [MIZ].

Podlasie Lowland: Mielnik [FD30], 11 VII 1975, 3 exx., leg. ZS, 29 VII 2008, 1 ex., leg. WJ [ISEA].

Lower Silesia: Świebodzice [WS93] ad Świdnica, 19 VI 1961, 5 exx.. leg. BB [MIZ].

Upper Silesia: Bytom [CA58], 16 VI 1978, 1 ex., leg. BI [USMB].

Kraków-Wieluń Upland: Czernichów [DA03], 27 VII 1882. 12 exx., leg. SS [ISEA]; Przeginia Duchowna. Kajasówka nat. res. [DA04]. 19 VII 1987, 1 ex.. leg. TW [ISEA]; KrakówZwierzyniec [DA14], 17 VIII 1919, 3 exx., leg. EM [MIZ]; Kraków-Kostrze [DA14]. 28 VI 1932, 8 exx.. leg. SS [ISEA]; Kraków-Podgórki [DA14], $10 \mathrm{~V}$ 1947, 11-18 VII 1954, 5 exx.. leg. JS \& WS [ISEA]; Kraków-Tyniec [DA14]. 2 VII 1991, 4 exx.. leg. MM [ISEA]: KrakówPrzegorzaly [DA14], 11 VII 1936, 3 exx., leg. SS [ISEA]: Kraków-Las Wolski [DA14]. 25 VII 1916, 8 exx.. leg. SS [ISEA]; Kraków: Panieńskie Skały [DA14], 22 VII 1920, 1 ex.. leg. TE [MIZ]: Kraków-Bielany [DA14]. 29 VII 1932. 1 ex., leg. SS. 15 IV-30 VII 1970. 2 exx.. leg. MG [ISEA]; Zabierzów, Skala Kmity [DA15], 1 VIII 1984, 1 ex., leg. PZ [ISEA]; Kraków-Mydlniki [DA24], 31 VII 1990, 1 ex., leg. MM [ISEA]. 
Malopolska Upland: Skorocice [DA78], 10 VII 1951, 29 exx., leg. JF, 30 V 1978, 2 exx.. leg. BB [MIZ]. 25 VII 1987. 14 VII 1996. 3 exx.. leg. DK \& HS [ISEA]. 14 VII 1996, 4 exx., leg. WZ [USMB]; Bogucice [DA79] ad Pińczów, 28 VII 1964. 2 exx.. leg. WS [ISEA]; Grabowiec nat. res. [DA79]. 12 VII 1953, 15 VII 1959. 3 VIII 1962. 17 exx., leg. BB [MIZ]: Chotel Czerwony [DA88] ad Busko, 4 VII 1961, 5 exx., leg. WS [ISEA]; Sandomierz [EB51], 6 VII 1919. 1 ex., leg. TE [MIZ]; Dwikozy [EB52] ad Sandomierz, 21 V 1955 and 30 VII 1956, 2 exx., leg. BB \& WB [MIZ]. 7 VII 1967, 8 exx.. leg. WS [ISEA]; Góry Wysokie [EB52]. 30 VII 1956. 1 ex., leg. EK \& WB [MIZ]; Janowiec [EB68] ad Kazimierz Dolny, 8 VII 2003, 1 ex., leg. DK [ISEA].

Świętokrzyskie Mts.: Chęciny [DB62], 17 VII 2009, 1 ex.. leg. DK [ISEA]; Nowa Shupia [EB03] ad Kielce, 20 VII 1967, 4 exx.. leg. WS [ISEA].

Lubelska Upland: Kazimierz Dolny [EB68], 8 VIII 1907, 1 ex., leg. TE [MIZ]. 6 VII 2002. leg. WJ [ISEA]: Dobre [EB68] ad Kazimierz Dolny. 30 VII 2004, 2 exx., leg. WJ [ISEA].

Sandomierska Lowland: Lubaczów [FA55], 4 VII 1968 and VII 1969, 3 exx.. leg. TN [USMB].

Eastern Sudety Mts.: Bardo Śląskic (Wartha) [XR29], 15 VII 1927, 2 exx., leg. KI [USMB].

Western Beskidy Mts.: Szczyrk [CA50]. VII 1952, 1 ex., leg. EI [MIZ].

A very widely distributed species in whole Europe, reaching also to Caucasus, Asia Minor and Siberia. KFP lists it under its synonymical name flavus (SCopoLI, 1763) with a comment that it probably occurs in the whole territory of Poland. It is fairly represented in the reviewed collections; the MIZ collection includes voucher specimens for notes of B. BurakowskI*. The ISEA and USMB collections contain specimens captured in four KFP regions (Podlasie Lowland, Kraków-Wieluń Upland, Lubelska Upland, Sandomierska Lowland) not listed in KFP.

\section{Cteniopus sulphuripes (GERMAR, 1824)}

Lower Silesia: Ligota Wielka (Ellguth Ottmachau) [XR49] ad Otmuchów. VII 1920. 2 exx.. leg. ED [USMB].

A species distributed from eastern Siberia and Turkey to Italy and France. It is rarely found in central Europe, in scattered xerothermic localities. All data regarding Poland come from the end of $19^{\text {th }}$ and beginning of $20^{\text {th }}$ centuries; it has not been observed in our country since then. Placed in Polish Red List with EN category (PAwlowski et al. 2002), but it would be advisable to confirm its present occurrence in Poland, or to consider its moving to EX? category.

\section{Omophlus pubescens (LinNaEus, 1758) [=betulae (Hersst, 1783)]}

Pomeranian Lake District: Szczecin (Stettin) [VV62], 25 IX 1924 and 21 V 1925, 3 exx.. leg. GL [MIZ]; Koszalin (Coeslin) [WA70]. date missing. 6 exx.. leg. AL [MIZ]; Grabowo [CE10]. Kozielec nat. res., 23 V 1995. 1 ex., leg. DK [ISEA]; Biała Góra [CE67] ad Sztum. 16 V 2003, 1 ex., leg. DK [ISEA]. 
Wielkopolska-Kujawy Lowland: Sieciejów [WT02], 4 VI 1983, 1 ex., leg. PS [ISEA]; Piastowskie Wzgórza Hills [WT35] ad Zielona Góra. 15-17 V 1955. 1 ex.. leg. SE [MIZ]; Nowa Sól (Neusalz) [WT43], 5 VI 1900, 1 ex., coll. MIZ: Puszczykowo [XT29] ad Poznań, 24 V 1951. 10 exx.. leg. RB [MIZ]; Włoclawek [CD63]. Kulin nat. res. vicinity, 13 V 2002. 1 ex.. leg. MM [ISEA]; Kretkowo [CD70], 1 VII 1938, 1 ex., leg. AB [MIZ].

Mazowiecka Lowland: Grodzisk Mazowiecki [DC77], 24 V 1931, 4 exx., leg. TE [MIZ]; Warszawa-Leszno [DC98], 13 V 1951. 1 ex., leg. BB [MIZ]; Jablonna [DD90]. 7-13 VI 1893. 3 exx., leg. WM [USMB]; Piaseczno [EC06], 3 VII 1908, 1 ex., leg. TE [MIZ]; Chojnów [EC06]. 8 VI 1924, 1 ex.. leg. TE. 16-17 V 1948, 2 exx., leg. ZW \& RB [MIZ]. 18 V 1970, 1 ex.. coll. MIZ; Konstancin [EC07], 23 V 1924 and 29 V 1930, 6 exx., leg. TE [MIZ]: Warszawa-Saska Kępa [EC08]. 21 V 1889. 1 ex., leg. WM [USMB]: Czersk [EC15], 26 V 1917. 1 ex., leg. JD [MIZ]; Świder [EC17], 15 VII 1900, 2 exx., coll. MIZ; Warszawa-Falenica [EC17], 23 V 1924. 1 ex.. leg. TE [MIZ]: Siennica [EC47]. 29 V 1932. 3 exx.. leg. AB [MIZ]: Zegrze [ED01]. 7 VI 1935, 1 ex.. leg. TE [MIZ]; Klembów [ED20]. 21 X 1956, 1-21 V 1958, 6 V 1962, 26 exx., leg. BB [MIZ]: „Warszawa”, 3 VI 1940, 1 ex., leg. TE [MIZ].

Podlasie Lowland: Łysów [FC19], 17 V 1953, 1 ex., leg. WB [MIZ]; Międzyrzec Podlaski [FC26]. date missing. 1 ex., leg. EI [MIZ]: Niemirów [FC49]. 13 V 2003, 1 ex.. leg. DK [ISEA]: Zajęczniki [FD10] ad Drohiczyn, 14 V 2003, 2 exx.. leg. MM [ISEA]; Puszcza Knyszyńska Forest: Woronicze nat. res. [FE60]. $17 \mathrm{~V}$ 1989, 1 ex.. leg. PO [ISEA].

Bialowieża Primeval Forest: Jakubowo [FD73]. 19 V 1993, 1 ex., leg. JG [ISEA]; f. comp. 117A [FD75]. $20 \mathrm{~V}$ 1993, 1 ex.. leg. JL [USMB].

Lower Silesia: Raszówka [WS88] ad Lubin, 28 V 1964, 3 exx., leg. RB [MIZ]; Kąty Opolskie (Konty) [YS10], 1 VI 1926, 1 ex.. leg. KI [USMB].

Kraków-Wieluń Upland: Opatów [CB44]. 19 X 1963, 1 ex.. leg. EI [MIZ]; KrakówPodgórki [DA14], 10 V 1947. 1 ex.., leg. WS [ISEA].

Malopolska Upland: Bogucice [DA79]. 26 V 1953. 1 ex.. leg. WS [ISEA]. 30 IV 1959. 1 ex., leg. WB [MIZ]; Ochojec [DB29]. 18 VI 1955, 1 ex., leg. SA [ISEA]; Nasilów [EB68] ad Kazimierz Dolny. 29 V 2003. 2 exx.. leg. DK [ISEA].

Świętokrzyskie Mts.: Kielce [DB73], 1-4 VI 1913, 2 exx., leg. EI [MIZ]; „Lysogóry”, 14 VI 1930, 1 ex., leg. AB [MIZ].

Lubelska Upland: Kazimierz Dolny [EB68], 17 V 1948, 15 V 1949, 17 V 1950, 4 exx.. leg. AR \& GO [MIZ]; Bochotnica [EB69], 23 V 1953. 1 ex., leg. WB [MIZ]; Goląb [EC60] ad Pulawy, 26 IV 1957, 2 exx., leg. BB [MIZ].

Roztocze Upland: Susiec [FA58]. 9 VI 1955, 3 exx., leg. BB [MIZ]; ad Narol [FA68]. 25 V 1967. 2 exx.. leg. JP [ISEA]; Florianka [FB30]. 24 VI 1905. 26 V 1907, 10 VIII 1916, 5 exx., leg. FF [MIZ]

Sandomierska Lowland: „Puszcza Niepolomicka Forest”, 6 VI 1912 and 14 VI 1914. 2 exx.. leg. TE [MIZ].

Western Beskidy Mts.: Kraków-Borek Falęcki [DA23], 31 V 1874, 2 exx., leg. BK [ISEA].

A widespread species all over Europe. According to KFP, where it has been listed as $O$. betulae (HERBST, 1783), it probably occurs in whole Poland except for high mountain areas. The MIZ collection includes numerous voucher specimens to faunistic notes signed by B. BurakowskI*. It is so far the only species from the genus Omophlus DAIL, which occurrence in Poland has been well documented. Other species mentioned 
by publication about 100 years ago are not represented in collections by particular specimens, thus they cannot be presently counted among Polish fauna.

\section{Podonta nigrita (FABRICIUs, 1794)}

Mazowiecka Lowland: Jabłoń [EC17], 8 V 1896, 1 ex.., leg. WM [USMB]; Świder [EC17]. 15 VII 1900. 2 exx.. coll. MIZ: Warszawa-Wawer [EC18]. 16 VI 1900. 2 exx.. leg. WM [USMB].

The species occurs in southern and eastern Europe, reaching Asia Minor and Caucasus in the south east. The beetles are captured on flowers of Asteraceae or Lamiaceae. Bionomy of this species is unknown. However, it is known to be connected with xerotherms, so its localities close to Warsaw (the collection of W. MĄcZYŃSKI) are doubtful. Also the explanation of MAKÓLSKI (1952), that single specimens of mountain beetles could be brought by Vistula waters to the vicinity of Warsaw, raises doubts in this case, especially that reports on occurrence of this species in Carpathians were published in the first half of the XX century, and data coming from publication of GERHARDT (1910) do not pertain to the territory of Poland (,.Beskiden" = „Lissa-Hora ", Zaolzie, Czech part of the Pogórze Cieszyńskie Mts.). The MIZ collection lacks the voucher specimen for the report of BuRAKOWSKI (1976) on occurrence of the species in the vicinity of Przemyśl. The species is now listed in Polish Red List in EN category (PAwŁOWsKI et al. 2002), however, its occurrence in Poland should be confirmed or it should be moved to EX category.

\section{DIAPERINAE LATREILLE, 1802}

\section{Diaperini LatreILLE, 1802}

\section{Alphitophagus bifasciatus (SAY, 1824)}

Masurian Lake District: Elk [EE86], 29 XII 1972, 3 exx.. leg. RB [MIZ].

Wielkopolska-Kujawy Lowland: Kościelna Wieś [BC94] ad Kalisz. 7 V 1990, 1 ex.. leg. AM [ISEA].

Western Beskidy Mts.: Polichty [DA91] ad Gromnik, 11 V 1999, 1 ex., leg. DK [ISEA].

A cosmopolitan species, occurring in whole Europe. Synanthropic in Poland (feeds on rotting straw, hay, decaying cereals and their products, infests stables, flour mills and storehouses) but also surviving outdoors (in molding broadleaf wood, under rotten bark and in hollows). The MIZ collection includes voucher specimens for faunistic records with B. Burakowski* signature (lack of specimens from Siennica, Mazowiecka Lowland). KFP lists occurrence of the species in seven regions. Publications that appeared after publication of Volume 14 of KFP give data on capturing the species in Bialowieża Primeval Forest (Kubisz \& Tsinkevich 2001), or in Upper Silesia (Melke \& Grzywocz 2003). 


\section{Diaperis boleti (LinNaEus, 1758)}

Pomeranian Lake District: rez. Bielinek nad Odrą [VU46], 28 IV 1953, 1 ex., leg. BB [MIZ], 30 V 1983, 6 exx., leg. DI [USMB]; Miastko [XV28]. 3 VI 1982, 1 VII 1983, 8 exx., leg. JM [ISEA]: Tleń [CE14]. 25 VIII 1922. 1 ex.. leg. TE [MIZ].

Masurian Lake District: Bachotek [CE91], 27 VII 1983, 6 exx., leg. BB [MIZ]; Orneta [DE49], 11 IX 1973, 1 ex.. leg. BB [MIZ]; Elk [EE86]. 25 X 1947. 3 exx.. RB [MIZ]; Sztynort [EF40]. 9 IX 1958, 2 exx.. leg. BB [MIZ]: Plociczno [FE29]. 14 XII 1954, 2 exx.. leg. BB [MIZ].

Wielkopolska-Kujawy Lowland: Sieraków [WU73], 23 VIII 1987, 1 ex.. leg. DI [USMB]; Rawicz (Rawitsch) |XT22|. VI 1903, 5 exx.. leg. FK [MIZ]: Czempiń [XT27]. 26 V 1997. 1 ex.. leg. WJ [ISEA]; Krajkowo [XT38], 1 VIII 1954, 1 ex., leg. BB [MIZ], 4 V 1984, 4 exx., leg. DI [USMB]; Rogalin [XT39], 14 IV 1984, 1 ex., leg. DI [USMB]: Sycyn Dolny [XU13], 23 V 1993. 1 ex.. leg. MA [ISEA]: Poznań-Dębina [XU30]. 27 V 1931. 1 ex.. leg. HC [MIZ]: Poznań [XU30], 22 V 1975, 1 ex., leg. JL [USMB]; Toruń: Kępa Bazarowa [CD37], 3 IX 1962, 1 ex.. leg. BB [MIZ].

Mazowiecka Lowland: Truskaw [DC89]. 22 IX 1968. 1 ex., leg. BB [MIZ]; WarszawaBuchnik [DC99]. 31 VII 1957, 1 ex., leg. BB [MIZ]: Warszawa-Mlociny [DC99], 13 I 1952. 3 exx.. leg. BB [MIZ]; Warszawa-Bielany [DC99], $18 \mathrm{VI}$ and 4 X 1888, 2 V 1890, 4 exx.. leg. WM [USMB]. 30 V-6 VI 1953, 3 exx., leg. RB. 17 V 1957. 1 ex.. leg. MZ. 15 VII 1950. 7 V and 31 VIII 1953, 25 VIII 1975, 5 exx., leg. BB [MIZ]; ad Modlin [DD70], 10 V 2001, 1 ex., leg. DK [ISEA]; Jabłonna [DD90], 1 VII 1893, 1 ex., leg. WM [USMB]: Warszawa-Wilanów [EC07], 18 IX 1917. 2 exx.. leg. TE [MIZ]: Warszawa-Natolin [EC07]. 23 IV 1939. 2 exx.. leg. TE [MIZ]: Warszawa-Morysinek [EC07]. 6 I 1937. 1 ex.. leg. EM [ISEA]. 6 I 1937. 2 exx.. leg. JF, 14 I 1951, 9 VI 1956, 6 I 1959, 5 exx., leg. BB [MIZ]: Warszawa: Zoological Garden [EC08], 13 VI 1940, 2 exx.. leg. TE [MIZ]: Dęblin [EC51]. 21 V 1925 and 30 VI 1929. 3 exx.. leg. HC [MIZ]: Struga [ED00]. 1 VI 1892, 2 exx., leg. WM [USMB]; Klembów [ED20], 27 VII 1983. 2 exx.. leg. BB [MIZ]; Urle [ED41], 8 VII 1909. 1 ex., leg. WM [USMB]; Dybki [ED44] ad Ostrów Mazowiecka, 26 V 1987. 1 ex.. leg. EN [MIZ]; „Warszawa”, 3 V 1895. 7 exx. leg. WM [USMB]. 10 VI 1917, 1 ex., leg. TE [MIZ].

Podlasie Lowland: Międzyrzec Podlaski [FC26], date missing, 3 exx., leg. EI [MIZ]; Niemirów [FC49] ad Mielnik, 13 V 2003, 2 exx.. leg. DK [ISEA]; Kopna Góra [FE60] ad Supraśl, 19 VIII 1985. 2 exx., leg. DK [ISEA].

Bialowieża Primeval Forest: Topilo, f. comp. 665D [FD73]. 25 VIII 2003, 1 ex., leg. EN [ISEA]: Grudki [FD78]. 2 VI 1982, 2 exx., leg. JG [USMB]: f. comp. 523/547 [FD84], 4 VI 1954, 1 ex.. leg. BB [MIZ]: Białowieża [FD94]. 11-13 VII 1920, 26 VI 1921. 8-14 VII 1930, 11 exx., leg. TE, 8 VI 1939. 1 ex., leg. HC, 24 IX 1951, 1 ex., leg. BB, 12 V 1968, 1 ex., leg. AD [MIZ]; Bialowieski N. P., f. comp. 398 [FD94]. 29 VIII 1968. 1 ex.. leg. BB [MIZ]; f. comp. 285 [FD95], 10 V 1959, 2 exx.. leg. BB [MIZ]: Siemianówka [FD96]. 9 IV 1976, 1 ex.. leg. JL [USMB].

Upper Silesia: Rybnik-Paruszowiec (Paruschowitz) [CA25], 22 IX 1911, 2 exx., leg. SC [USMB]; Tarnowskie Góry (Tarnowitz) [CA49]. 14 V 1911. 5 exx.. leg. GP [USMB]; Tarnowskie Góry-Pniowiec (Pniow) [CA49], 1 VI 1934, 1 ex., leg. KI [USMB]; Pszczyna (Pless) [CA53]. 8 VII 1923, 1 ex., coll. USMB; Ruda Śląska-Kochłowice [CA57], 9 V 1939, 1 ex.. leg. KW [USMB]: Oświęcim-Bobrek [CA74]. 13 X 1999. 5 exx., leg. RD [USMB]: Ciężkowice [CA86] ad Jaworzno. 5 IV 1920, 7 exx.. leg. SS [ISEA]; Byczyna [CB06], 14 V 1961. 5 exx., leg. SI [USMB]: Zawadzkie [CB20], 2 XI 1930, 1 ex., leg. HN [USMB]; Kościeliska (Hedwigstein) 
[CB25] ad Olesno. 8 VII 1923, 1 ex., coll. USMB; Lubliniec [CB31]. 12 IX 1993, 4 exx.. leg. $\mathrm{RD}$ [USMB].

Kraków-Wieluń Upland: Kraków [DA24], 1 X 1984, 1 ex., leg. PA [ISEA].

Malopolska Upland: Polana Polichno nat. res. [DA69]. 18 V 1994, 1 ex.. MA [ISEA]; Spala [DC30], 29 VI 1912, 2 exx., leg. EI [MIZ].

Świętokrzyskie Mts.:Łysica Mt. [DB93], 21 VI 1897, 2 exx.. leg. SS [ISEA]; Łysa Góra Mt. [EB03]. 2 VI 1956, 2 exx.. leg. BB [MIZ]; „Świętokrzyskie Mountains”, 15-29 V 1957. 2 exx., leg. PD [MIZ].

Lubelska Upland: Kazimierz Dolny [EB68], 11 VIII 1907, 1 ex., leg. TE, 13 V 1949. 8 exx., leg. AR \& GO [MIZ]; Pulawy [EB69], date missing, 1 ex., leg. HC. 16 VII and 9 VIII 1950, 8 exx.. leg. BB [MIZ]; Parchatka [EB69]. 9 VIII 1950. 3 exx., leg. BB [MIZ]; Macoszyn [FB79]. 21-30 V 1993, $20 \mathrm{~V}$ 1994, 2 exx., leg. MA [ISEA].

Roztocze Upland: Ulów [FA58]. 15 VIII 1912, 14 exx., leg. TE [MIZ]; Zwierzyniec [FB30]. 22 VII 1911, 2 exx., leg. TE, 15 VI 1914, 1 ex., leg. FF [MIZ]: Florianka [FB30]. 18 V 1905. 2 exx.. leg. FF [MIZ]: Nart [FB50]. 14 VII 1910, 9 exx.. leg. TE [MIZ].

Sandomierska Lowland: Wierzchoslawice [DA94] ad Tarnów, 7 VIII 1917, 10 exx., leg. SS [ISEA]; Zaleszany [EB61] ad Sandomierz. 1-10 IX 1890. 2 exx.. leg. SS [ISEA].

Western Beskidy Mts.: Cieszyn [CA21]. 1874-1882, 1 ex.. leg. BK [ISEA]; Czantoria Mt. [CA40]. 1880. 2 exx., leg. BK [ISEA]: Równica Mt. [CA40], 28-29 VI 1876, 1 ex., leg. BK [ISEA]: Wisla [CA40]. V 1935, 9 exx., leg. WM [USMB]: Barania Góra Mt. [CV59]. VII 1876. 1 ex.. leg. BK [ISEA]; Zawoja [CV99]. 29 VI-27 VII 1879, 24-27 VII 1880, 14 exx.. leg. SS. 7-25 VIII 1936, 27 VII-20 VIII 1937, 7-11 VII 1938, 110 exx., leg. SP [ISEA]; Zawoja-Barańcowa [CV99]. 15-26 VII 1948, 18 exx.., leg. ZW, 21 IV 1949, 8 exx., leg. RB [USMB]. 24-29 VII 1949. 8 exx.. leg. GO [MIZ]: Babia Góra Mt. [CV99]. 26 VI-1 VIII 1879. 14 VII 1880. 31 exx.. leg. SS [ISEA]. VII 1919. 2 exx., leg. EM, 26 VII 1949, 1 ex., leg. GO [MIZ], 1 XI 1949, 3 exx., leg. RB [USMB]: Młodów [DV77] ad Rytro. 12 V 1899. 3 exx.. leg. SS [ISEA]: Rytro [DV78]. 14 VII 1879, 20 V-16 VIII 1892, 16 V-21 VI 1896, 10 VII-18 IX 1898, 12 IV-7 VIII 1899, 6 VII 1901. 19 VI 1919. 179 exx.. leg. SS [ISEA]. 22 V 1924, 5 exx.. leg. EM [MIZ]: Nowy Sacz [DV79]. 21 VII 1892, 5 exx.. leg. SS [ISEA]; Żegiestów [DV87], 15 VIII 1892, 4 exx., leg. SS [ISEA]: Ptaszkowa [DV99] ad Grybów. 30 VI 1892, 3 exx.. leg. SS [ISEA]; Dębowa [EA22] ad Jodlowa. 30 VI 1999, 1 ex., leg. DK [ISEA].

Eastern Beskidy Mts.: Panieński Czub [FA00] ad Bircza, 4-22 V 1879, 16 exx.. leg. BK [ISEA]; Góra Biala (Splawa) [FA10]. 7 VI-6 VII 1884, 3 exx., leg. BK [ISEA]; Olszany [FA11] ad Przemyśl. 1887-1878, 3 exx., leg. BK [ISEA]; Przemyśl: Budy [FA21]. VI-VII 1881, 1 ex.. leg. BK [ISEA].

Bieszczady Mts.: Lutowiska [FV25]. 28 VI 1953, 1 ex., leg. BB [MIZ].

Pieniny Mts.: Czorsztyn [DV47]. VI 1952, 1 ex.. leg. KA [ISEA].

Tatry Mts.: Czarny Staw Gąsienicowy [DV25], 13 VII 1893, 1 ex.. leg. SS [ISEA]; Bystra Valley [DV25], 24 VI 1900, 5 exx.. leg. MR [ISEA].

A species widespread everywhere in Europe. It inhabits sporocarps of Polyporaceae growing mainly on broadleaved trees, sometimes on coniferous trees. It occurs in all territory of Poland and it is one of most frequently captured darkling beetles. Very numerous specimens in the reviewed collection. It is a mere formality to state that it occurs also in regions not listed in KFP (Podlasie Lowland, Sandomierska Lowland, Małopolska Upland, Pieniny Mts. and Tatry Mts.) on the basis of specimens deposited 
mainly in ISEA collection (specimens collected in localities in Nowotarska Dale are missing).

\section{Gnatocerus cornutus (FABRICIUS, 1798)}

Wielkopolska-Kujawy Lowland: Łęczyca [CC76], 8 X and 16 XI 1935. 8 exx.. leg. AB [MIZ]: Kolo [CC38], 15 I 2000, 1 ex.. leg. MK [ISEA].

Mazowiecka Lowland: Świder [EC17]. 15 VII 1900. 2 exx.. leg. WM [USMB]; „Warszawa”. IV-V 1916, 7-15 V 1929, 29 VIII 1939. 8 exx., leg. TE [MIZ].

Lower Silesia: Zloty Stok (Reichenstein) [XR39]. 4 IV 1931. 1 ex.. leg. KI [USMB].

Upper Silesia: Bytom [CA58], 25 VI 1984, 10 exx., leg. MD [USMB].

Kraków-Wieluń Upland: Kraków. Krowoderska str. [DA24], ca. 1900. 5 exx.. leg. MR [ISEA].

Malopolska Upland: Łódź [CC93]. from flour. 20 IX 1980. 1 ex.. leg. TM [ISEA].

Sandomierska Lowland: Bobrówka [FA34] ad Jarosław, 15 VII-15 VIII 1960, 13 exx., leg. SO [USMB].

Although it is a cosmopolitan species, constantly introduced to Europe in food products, it is little known in Poland (Burakowski et al. 1987). It is considered pest of cereals and their products. In KFP, only about a dozen reports from 6 regions are supported. A voucher specimen for a note signed B. BuRAkowski* (Lęczyca) is deposited in the MIZ collection. The ISEA collection contains a specimen collected in Lódź (Malopolska Upland), and the USMB collection - specimens captured in Upper Silesia and Sandomierska Lowland (regions not listed in KFP).

\section{Neomida haemorrhoidalis (FABRICIUS, 1787)}

Masurian Lake District: Kulik [EE43] ad Pisz, 22 X 1962, 2 exx., leg. BB [MIZ]; f. distr. Boczki [FF02] ad Goldap, 29 III 1955, 1 ex., leg. BB [MIZ].

Podlasie Lowland: Chotylów [FC66]. 10 VI 1935, 1 ex.. leg. HC [MIZ]; Kopna Góra [FE60] ad Supraś1, 20 VIII 1985, 1 ex., leg. PT [ISEA].

Mazowiecka Lowland: Świder [EC17]. 15 VII 1900. 5 exx.. leg. WM [USMB].

Bialowieża Primeval Forest: Topilo, f. comp. 665D [FD73], 25 VIII 2003, 2 exx., leg. EN [ISEA]; Podcerkwa [FD83]. 2 XII 2001. 1 ex.. leg. RD [USMB]; Bialowieski N. P.. f. comp. 340 [FD94]. 19 IV 1967, 1 ex., leg. BB [MIZ]; Białowicża [FD94]. 9 VII 1930, 5 exx., leg. TE. $30 \mathrm{~V}$ 1934, 19 III 1937, 8 VI 1939, 7 exx.. leg. HC. 23 IX-1 X 1950, 2 exx., leg. BB [MIZ], 4 V 1987, 2 exx., leg. DK [ISEA]: Bialowieski N. P., f. comp. 314 [FD95], 19 IV 1967, 1 ex., leg. $\mathrm{BB}$ [MIZ].

Upper Silesia: Rybnik [CA25]. 12 VII 1934, 9 exx., leg. KL [MIZ]; Katowice-Murcki (Emmanuelssegen) [CA56]. 13 XI 1928, 20 XI 1929, 23 IV and 21 V 1930, 10 exx., leg. HN, 6-20 VI 1928, 8 exx.. 20 XI 1929, 12 exx.. 21 V 1930, 14 exx., leg. KI [USMB].

Kraków-Wieluń Upland: Miękinia [DA05]. 28 IV 1912, 1 ex.. leg. TE [MIZ].

Roztocze Upland: Kosobudy-Katy [FB41]. 27 VI 1902. 1 ex.. leg. HO [MIZ].

Western Beskidy Mts.: Rytro [DV78]. 19 VI 1892, 1-8 VI 1901, 10 VI-10 VII 1902. 9 exx.. leg. SS [ISEA]. 
Eastern Beskidy Mts.: Panieński Czub [FA00] ad Bircza, 22 V 1879, 25 exx., leg. BK [ISEA]: Góra Biała (Splawa) [FA10], 7 VI-6 VII 1884, 6 exx., leg. BK [ISEA]; Hołubla [FA11]. 25 V-29 VI 1884, 65 exx., leg. BK [ISEA]: Olszany [FA11] ad Przemyśl. 1877-1878, 7 exx.. leg. BK [ISEA]; Kamienna Laworta Mt. [FV17] ad Ustrzyki Dolne, 19 VI 1880, 2 exx.., leg. BK [ISEA].

Bieszczady Mts.: Wetlina [FV04]. 28 VI 1954, 1 ex.. leg. BB [MIZ]. 16 IX 1963. 2 exx.. leg. SZ [ISEA]: Terebowiec [FV23], 27 VI 1953, 1 ex., BB [MIZ].

Pieniny Mts.: Droga Pienińska [DV57]. 17 VIII 1939, 1 ex., leg. TE [MIZ].

Euro-Siberian species, considered a relict of old-growth forest, more and more rare in central Europe. According to KFP, the species was recorded in seven regions. Further publications (Borowiec 1991; Kubisz \& Szwalko 1991; Kubisz et al. 1998; Pawlowski et al. 2000; Kubisz \& Tsinkevich 2001; Gutowski et al. 2006; Bidas 2007; Gawroński \& Oleksa 2007; Bunalski et al. 2010; Gutowski et al. 2010; MarczaK 2010) brought documented reports of not only occurrence of this species in Poland, but also new localities in four other KFP regions (Wielkopolska-Kujawy Lowland, Mazowiecka Lowland, Podlasie Lowland, Świętokrzyskie Mts., Bieszczady Mts.). MIZ collection includes a specimen captured in 1912 in Kraków-Wieluń Upland (the species has not been reported from that KFP region so far). Neomida haemorrhoidalis is listed in Polish Red List (Pawlowski et al. 2002), NT category.

\section{Pentaphyllus testaceus (HeLlwig, 1792)}

Pomeranian Lake District: Szczecin (Stettin) [VV62], date missing, 2 exx., coll. MIZ.

Masurian Lake District: Sztynort [EF40]. 9 VIII 1958. 3 exx.. leg. BB [MIZ]; Bryzgiel [FE38] ad Augustów, 12 VIII 1955, 3 exx., leg. BB [MIZ].

Wielkopolska-Kujawy Lowland: Osowa Góra [XU99], 12 IV 1958, 2 exx.. leg. BB [MIZ]; Łęczyca [CC76]. 5 IX 1937. 1 ex.. leg. AB [MIZ]: Toruń [CD37]. 22 III 1936, 1 ex.. leg. AB [MIZ]; Toruń: Kępa Bazarowa [CD37]. 21 IX 1956, 5 exx.. leg. BB [MIZ].

Mazowiecka Lowland: Skierniewice [DC45]. 22 V 1901. 2 exx., leg. WM [USMB]; Puszcza Kampinoska Forest: Zamczysko [DC69]. 25 I 1953, 2 exx.. leg. BB [MIZ]; Podkowa Leśna [DC87], 13 VII 1934, 2 exx., leg. TE [MIZ]; Warszawa-Bielany [DC99], 27 VI 1888, 12-24 $\mathrm{V}$ and $14 \mathrm{X}$ 1889, 11 exx., leg. WM [USMB]. 1 XI 1936 and 1 IX 1938, 8 exx., leg. AB, date missing. 1 ex.. leg. EM. 22 I 1950, 1 I 1951, 23 I. 22 XI. 26 XII 1953. 26 IX 1954, 29 I and 13 II 1955, 4-23 II 1959, 7 II and 2 VII 1960, 8 V 1965, 27 II 1966, 18 III 1979, 4 VII 1988, 47 exx., leg. BB [MIZ]; Warszawa: Zoological Garden [EC08], 13 VI 1940, 3 exx., leg. TE [MIZ]; Dhuga Kościelna [EC28]. 5 XI 1950, 4 exx.. leg. BB [MIZ]; Gliniak [EC37] ad Mińsk Mazowiecki. 4 VII 1923, 1 ex., leg. HC [MIZ]; Klembów [ED20], 29 IX 1951. 2 exx., leg. BB [MIZ]: Urle [ED41], 13 VIII 1910, 1 ex., leg. TE [MIZ].

Lower Silesia: Wroclaw: Park Szczytnicki [XS46], 17 VI 1961, 13 exx., leg. BB [MIZ]; Wroclaw-Niskie Łaki [XS46], 8 VII 1990, 2 exx.. leg. RK [ISEA].

Upper Silesia: rez. Łężczak [CA05], 28 VIII 1996, 1 ex., leg. ST [ISEA]; Polomia [CA39]. 29 X 1999. 1 ex.. leg. RD \& HS [USMB].

Kraków-Wieluń Upland: Kraków-Dąbie [DA24], ca. 1900, 15 exx.. leg. MR [ISEA]; „Kraków vicinity”, 1914, 1 ex.. leg. TE [MIZ].

Western Beskidy Mts.: Zawoja-Markowa [CV99], 12 VIII 1879. 1 ex.. leg. SS [ISEA]. 
European species, widely distributed, but rather rarely met in Poland. It dwells in dry, rotting wood of broadleaved trees, especially in trunks of oak infested by parasite fungus, sulphur shelf - Laetiporus sulphureus (BulL. ex Fr.) Murr. The majority of the published data come from the first half of the XX century, when many specimens from the reviewed collections were captured. The MIZ collection includes voucher specimens for faunistic reports signed B. BuRAKOWSKI*.

\section{Platydema dejeanii Laporte \& Brullè, 1831}

Mazowiecka Lowland: Świder [EC17], 15 VII 1900, 3 exx.. leg. WM [USMB]; Siennica [EC47], $19^{\text {th }}$ century, 3 exx.., leg. AW [ISEA].

Bialowieża Primeval Forest: f. comp. 730 [FD83]. 1 VIII 1988. 1 ex.. leg. JG [ISEA]; f. comp. 340 [FD94]. 6 IV 1955, 31 III 1966, 3 exx.. leg. BB [MIZ]: f. comp. 370 [FD94]. 5 V-6 VI 1954, 7 exx.., leg. BB [MIZ]: f. comp. 373 [FD94], 29 VIII 1968, 1 ex., leg. BB [MIZ]: f. comp. 375 [FD94]. 17 VIII 1999. 1 ex.. leg. JG [ISEA]: f. comp. 397 [FD94]. 5 IV 1955. 3 exx.. leg. BB [MIZ]; f. comp. 475 [FD94], 12 VIII 1957, 4 exx.. leg. BB [MIZ]; f. comp. 476 [FD94], 12 VIII 1957, 1 ex., leg. BB [MIZ]; f. comp. 477 [FD94], 12 VIII 1957, 1 ex., leg. BB [MIZ]: Białowieża [FD94]. 25 IX 1951. 1 ex.. leg. BB [MIZ]: f. comp. 192 [FD95]. 10 V 1959. 1 ex.. leg. BB [MIZ]; f. comp. 286 [FD95]. 7 VI 1968, 1 ex.. leg. AD [MIZ].

Lubelska Upland: Kazimierz Dolny [EB68], 29 V 1939, 1 ex., leg. HC [MIZ].

Roztocze Upland: Zwierzyniec [FB30]. 20 IX 1920. 1 ex.. leg. FF [MIZ]; Zwierzyniec [FB30]. Bukowa Góra, 10 VI 1989, 1 ex.. leg. DI [USMB]: Panasówka [FB30]. 20 VIII 1911. 1 ex., leg. TE [MIZ]; f. distr. Grele [FB40]. 14 VIII 1911, 4 exx.., leg. FF [MIZ]: Jarugi [FB41]. 29 III 1990. 1 ex.. leg. LB [ISEA]: Nart [FB50]. 15 VII 1910, 1 ex.. leg. TE [MIZ].

Western Beskidy Mts.: Makowica Mt. [DV78] ad Rytro. 3 VIII 1939. 5 exx., leg. AB \& BB [MIZ].

Eastern Beskidy Mts.: Hohubla [FA11]. 25 V 1884. VI 1886, 17 V 1879, 12 exx.. leg. BK [ISEA]; Kaczmarowa Mt. [FA21] ad Łętownia. 28 IV 1879. 1 ex.. leg. BK [ISEA]; PrzemyślPralkowce [FA21], 8 V 1883, 18 V 1884, 27 IV-7 V 1879, 9 exx., leg. BK [ISEA]: Rokszyce [FA21] ad Przemyśl. 24 IX 1962, 1 ex.. leg. BB [MIZ].

Euro-Siberian species, a great rarity in central Europe and in Poland. The reviewed collections contain specimens captured in all five KFP regions (listed in KFP), including voucher specimens for faunistic notes by B. BuRAKowski*. The report of MARCZAK (2010) on occurrence of $N$. haemorrhoidalis in Kampinoski National Park supports also the possibility of the occurrence of $P$. dejeanii (three specimens from Siennica, $19^{\text {th }}$ century, from A. WAGA's collection [ISEA], and three specimens from Świder deposited in USMB, in the collection of W. MączYŃski). An identical label (Świder, 15 VII 1900) is attached to specimens of $N$. haemorrhoidalis preserved in USMB, the collection of W. MĄCZYŃsKI. MAKÓLSKI (1952) admits that it is possible that species connected with old-growth forests occurred in the vicinity of Warsaw at the turn of $19^{\text {th }}$ and $20^{\text {th }}$ centuries (the period of gathering the entomological collection by W. MĄczYŃskI). Their absence nowadays can be explained by destruction of large areas of forest, especially on the right side of the Vistula river, and removal of moribund and fallen trees. Due to its extreme rarity and habitat (oldgrowth forests), it is advisable to consider introducing this species on the Polish Red List. 


\section{Platydema violaceum (FABRICIUS, 1790)}

Pomeranian Lake District: Bielinek nad Odrą [VU46], 29 IX 1984, 1 ex., leg. DI [USMB].

Wielkopolska-Kujawy Lowland: Goluchów [YT05] ad Kalisz. 20 IV 1984. 1 XI 1990. 2 exx.. leg. AM [ISEA]: Krajkowo [XT38] ad Poznań. 25 VIII 1988, 1 ex.. leg. DK [ISEA]: Brączewo [XU04] ad Obrzycko, 26 XI 1985, 5 exx., leg. KZ [ISEA. USMB]: Toruń [CD37], 31 X 1937, 2 exx., leg. AB [MIZ].

Mazowiecka Lowland: Warszawa-Bielany [DC99], 17 IV 1885. 1 ex., leg. WM [USMB]; Warszawa-Wawer [EC18]. 2 IV 1905, 1 ex., leg. TE [MIZ].

Podlasie Lowland: Marcelin [ED50]. 7 VII 1894. 3 exx., leg. WM [USMB].

Bialowieża Primeval Forest: Białowieża [FD94], 19 III 1952 and 5 IV 1955, 2 exx., leg. $\mathrm{BB}$ [MIZ].

Trzebnickie Hills: Trzebnica [XS49]. 31 XII 1975, 1 ex., leg. J [USMB].

Kraków-Wieluń Upland: Kraków-Skały Panieńskie [DA14], 22 XI 1872, 1 ex., leg. BK [ISEA]; Kraków-Wola Justowska [DA14], 2 VI 1904, 2 exx., leg. SS [ISEA]; Kraków-Las Wolski [DA14]. 13 IV 1986, 5 X 1988, 2 exx., leg. DK [ISEA]: Ojców [DA16]. 15 VI 1982, 1 ex.. leg. JM [ISEA]: Kraków-Dąbie [DA24]. V 1989. 1 ex., leg. JM [ISEA]: Mlodziejowice [DA25]. 11 III 2006, 3 exx., leg. LP [ISEA].

Malopolska Upland: Będków [CB67], 3 V 1975, 2 exx., leg. JL [USMB]; Rogów [DC24] ad Koluszki. 11 XI 1954. 1 ex.. leg. BB [MIZ].

Roztocze Upland: Zamość [FB52], VII 1930, 1 ex., leg. FF [MIZ].

Sandomierska Lowland: Bobrówka [FA34] ad Jarosław, 15 VII-15 VIII 1960, 1 ex., leg. SO [USMB].

Western Beskidy Mts.: Wojkowa [EV06] ad Krynica. 8 V 1984. 1 ex.. leg. SD [ISEA].

The species occurs in central Europe, but is met rarely and sporadically. Both adults and larvae feed on sporocarps of Auriculariaceae family, growing on live and dead trees. It is known in Poland from few localities in more than a half of KFP regions. However, most of the reports come from publications dating back to the $19^{\text {th }}$ and the first half of the $20^{\text {th }}$ century. In the studied collections, there are specimens captured in the territory of seven KFP regions, both in the $19^{\text {th }}$ century (Kraków-Skaly Panieńskie, 1872) an in the second half of the $20^{\text {th }}$ century. The collections includes voucher specimens captured in Sandomierska Lowland and in Western Beskidy Mountains (not listed in KFP).

\section{Crypticini Brullé, 1832}

\section{Crypticus quisquilius (Linnaeus, 1761)}

Baltic Coast: Trzciagowo [VV57] ad Wolin. 14 VII 1975, 1 ex., leg. PS [ISEA]; Rowy [XA35] ad Ustka. 19-21 VII 1970, 2 exx., leg. JP [ISEA]; Łeba [XA66], 20-25 VI 1987. 20 VI 1993, 3 exx., leg. JP \& PZ [ISEA]; Rąbek [XA67] ad Łeba, 9 VIII 2000, 1 ex., leg. ST [ISEA]: Jastrzębia Góra [CF27], 19 VIII 1936. 1 ex.. leg. SM [MIZ]: Władyslawowo [CF37], 6-18 VI 1993. 10 exx.. leg. RD [USMB]; Sopot [CF43]. VII 1952. 1 ex.. leg. EI [MIZ]; Hel [CF55], 22 VII 1927. 7 exx., leg. SS [ISEA]. 
Pomeranian Lake District: Bielinek nad Odrą [VU46], 30 V 1984, 1 ex., leg. DI [USMB]; Szczecin-Gumieńce (Scheune b. Stettin) [VV62]. 6 VI 1917, 1 ex.. leg. PN [MIZ]; Szczecin (Stettin) [VV71]. 18 VI 1930, 2 exx., coll. MIZ; Koszalin (Coeslin) [WA70], 10 exx.. leg. AL [MIZ]; Bielawy [XU69] ad Naklo, 17 V 2002, 1 ex.. leg. DK [ISEA]; Topolno [CE11] ad Gruczno, 30 V 1996, 1 ex., leg. DK [ISEA]: Tleń [CE14], 31 VII 1922. 4 exx., leg. TE [MIZ]: Wzgórza Plutowskie Hills [CE20]. 27 V 1996, 1 ex.. leg. DK [ISEA].

Masurian Lake District: f. distr. Śmieszny Kąt [DE35] ad Stare Jablonki. 7 VI 2004, 1 ex., leg. EN [ISEA]: Suwalki [FE29], 2 VI 1918, 3 exx., leg. FK [MIZ].

Wielkopolska-Kujawy Lowland: Nowa Sól (Neusalz) [WT43]. 1 VIII 1907. 2 exx.. coll. MIZ: Slawa (Schlawa) [WT74]. VI 1937, 1 ex., coll. USMB; ad Kalisz [BC94], 15 VI 1988. 1 ex., leg. AM [ISEA]; Opatówek [CC03] ad Kalisz. 5 VII 1985, 1 ex., leg. AM [ISEA]; Kolo [CC38]. 6 VII 1958. 2 exx.. leg. BB [MIZ]: Łęczyca [CC76]. 15 V 1931. 10 VI 1935. 11 IX 1936, 3 exx., leg. AB [MIZ]: Barlogi [CC49], 29 V 1975, 1 ex.. leg. LB [USMB]: Borek [CD18] ad Toruń, 25 VI-18 VIII 1964, 2 exx., leg. WS [ISEA]; Ciechocinek [CD55], 16 VII 1918, 2 exx., leg. TE. 18 VI 1950, 2 exx., leg. BB [MIZ]: Miszek [CD55]. 16 VI 1986. 1 ex.. leg. JG [USMB]: Sycyn Dolny [XU13] ad Szamotuly, 16 VI 1991, 2 exx., leg. MA [ISEA]; Wierzonka [XU41]. 10-20 VI 1947, 5 exx., leg. WE [MIZ]; Zielonka [XU42] ad Poznań, 25 VI 1974, 2 exx.. leg. PS [USMB]: Niezgoda [XU90]. 10 VI 1964, 4 exx.. leg. AN [USMB]: „Poznań”, 28 V 1930. 1 ex.. leg. HC [MIZ].

Mazowiecka Lowland: Plecewice nad Bzurą [DC59], 12 VI 1952, 5 exx., leg. BB [MIZ]; Grodzisk Mazowiecki [DC77]. 4 VII 1965. 1 ex.. leg. BB [MIZ]: Brwinów [DC77]. 29 VI 1949. 2 exx., leg. AR [MIZ]; Grabina [DC79] ad Warszawa, 8 V 1950, 1 ex., leg. BB [MIZ]; Podkowa Leśna [DC87], 26 V 1949, 1 ex., leg. AD [MIZ]; Kampinoski N. P.. Lipków [DC89], 26 V 2003, 2 exx., leg. WJ [ISEA]: Sieraków [DC89]. 31 V 1953, 1 ex.. leg. BB [MIZ]: WarszawaSlużewiec [DC98]. 20 VII 1908, 1 ex., leg. TE [MIZ]; Warszawa-Wlochy [DC98]. 1 ex., leg. TE [MIZ]; Łomianki [DC99], 24 VII 1910, 1 ex., leg. TE [MIZ]; Warszawa-Mlociny [DC99]. 28 V 1918, 10 exx., leg. TE [MIZ]: Warszawa-Bielany [DC99], 4 VII 1888 and 9 V 1891. 2 exx.. leg. WM [USMB]. 13 VIII 1962, 1 ex., leg. BB [MIZ]: Pomiechówek [DD71]. 13 VI 1928, 2 exx. leg. AB [MIZ]; Jablonna [DD90], 16-30 V and 4 VI 1893, 3 exx.. leg. WM [USMB]: Piaseczno [EC06], 9 VII 1908, 1 ex.. leg. TE [MIZ]; Chojnów [EC06], 18 V 1920, 1 ex.. coll. MIZ: Żabieniec [EC06], 21 VI 1909. 4 exx., leg. TE [MIZ]; Warszawa-Wilanów [EC07]. 8 IX 1915. 3 exx., leg. TE [MIZ]; Warszawa: Zoological Garden [EC08]. 29 V 1940, 1 ex.. leg. TE [MIZ]; Pustelnik [EC09], 11 VI 1917, 2 exx.. leg. TE [MIZ]; Drewnica [EC09], 17 VI 1917, 4 exx., leg. TE [MIZ]: Warka [EC13]. 22 VI 1952, 1 ex., leg. BB [MIZ]: Świder [EC17], 12 VI 1904. 1 ex.. leg. WM [USMB]; Warszawa-Anin [EC18], 14-26 VII 1949 and $30 \mathrm{~V}$ 1950, 3 exx., leg. KG \& RB [MIZ]: Warszawa-Zbójna Góra [EC18]. 1 VI 1961. 1 ex.. leg. WB [MIZ]; Żwir [EC18]. 5 VI 1922, 2 exx., leg. HC. 15 VI 1951, 1 ex., leg. BR [MIZ]: Zielonka [EC19]. 8 VI 1949. 1 ex.. leg. ZW [MIZ]; Celestynów [EC26], 14-28 V 1950, 4 exx., leg. BB [MIZ]; Dęblin [EC51], 13 V 1925, 2 exx., leg. HC [MIZ]; Karasiówka [EC61], 8 VII 1908, 1 ex., leg. TE [MIZ]; Czarna Struga [ED00], 30 V 1915, 1 ex., leg. TE [MIZ]; Popowo [ED02] ad Serock. 24 V 2003, 1 ex.. leg. WJ [ISEA]; Kalinowo [ED65], 14 VII 1933, 1 ex., leg. AB [MIZ]; „Warszawa”, 3 V 1895. 4 exx.. leg. WM [USMB].

Podlasie Lowland: Mocarze [EE90] ad Jedwabne, 24 V 1969, 1ex., leg. JP [ISEA]; Międzyrzec Podlaski [FC26], date missing. 2 exx., leg. EI [MIZ]; Niemirów [FC49], 15 VI 2003, 1 ex.. leg. MM [ISEA]; Wólka Zamkowa [FD10] ad Drohiczyn, 21 VI 2001, 1 ex.. leg. DK [ISEA]: Zajęczniki [FD10] ad Drohiczyn. 15 VI 2003. 1 ex.. leg. DK [ISEA]: Mielnik [FD30] ad Siemiatycze, 30 V 1993, 1 ex.. leg. JG. 22 VI 2001. 1 ex.. leg. MM [ISEA]. 
Bialowieża Primeval Forest: Podborowisko [FD76]. 21 VI 1993, 1 ex., leg. JG [ISEA]; Gruszki [FD85]. 25 VI 1990, 1 ex., leg. DK [ISEA]: Białowieża [FD94]. 8 VII 1930, 2 exx., leg. TE [MIZ]; „Bialowieża Primeval Forest”. 14 VI 1937. 1 ex.. leg. AB [MIZ].

Lower Silesia: Świdnica [XS03]. 26 VII 1965. 3 exx., coll. USMB.

Upper Silesia: Rybnik-Paruszowiec (Paruschowitz) [CA25]. 3 VIII 1909, 1 ex.. leg. SC [USMB]; Pniów [CA28] ad Pyskowice. 16 VI 1929. 1 ex.. leg. KI [USMB]; Przezchlebie [CA38], 8 VI 1926, 1 ex., leg. KI [USMB]; Gliwice-Łabędy (Laband) [CA38], 28 II 1928. 3 exx.. coll. USMB; Orzesze [CA45]. VI 1923, 2 exx., leg. WY [USMB]: Ruda Śląska (Ruda) [CA46]. 3 VI 1923, 1 ex.. coll. USMB: Bielszowice (Bielschowitz) [CA47]. 6 VII 1924. 2 exx.. coll. USMB; Tarnowskie Góry-Pniowiec (Pniow) [CA49]. 1 VI 1934. 3 exx., leg. KI [USMB]; Bytom (Beuthen) [CA58], 8 IV 1913, 1 ex., leg. KI [USMB]: Chełmek [CA75]. 8 VII-10 VIII 1878, 1 VII 1880, 6 exx., leg. SS [ISEA]: Jaworzno-Szczakowa [CA76]. 11 VII 1910. 27 V9 VI 1911. 3 exx., leg. SP [ISEA]: Dąbrowa Górnicza [CA77], V 1934, 3 exx.. leg. WM [USMB]: Żarki [CA84] ad Chrzanów. 20 V-20 VI 1881, 4 exx., leg. SS [ISEA]; Pogorzyce [CA85] ad Libiąż, 2 VII 1880, 1 ex. leg. SS [ISEA]; Wojciechów (Albrechtsdorf) [CB14] ad Olesno. 10 VIII 1938, 3 exx.. leg. HN [USMB]; Lubliniec [CB31], 27-28 VI 1994, 2 exx., leg. RD [USMB]: Woźniki [CB60] ad Lubliniec. 4 IX 1937, 1 ex., leg. WM [USMB].

Kraków-Wieluń Upland: Klucze [CA97]. 22 VI 1994, 1 ex.. leg. DK [ISEA]; Cieciulów [CB35]. 29 V 1937, 1 ex., leg. WM [USMB]; Klobuck [CB54], 15 VII 1924, 1 ex., leg. HC [MIZ]; Czernichów [DA03]. VII 1912, 1 ex., leg. EM [MIZ]; Liszki [DA14] ad Kraków, date missing. 1 ex.. coll. ISEA; Kraków-Sikornik [DA14]. 26 V-3 VII 1935. 12 VI 1936, 3 exx.. leg. SP [ISEA]; Kraków-Zakrzówek [DA14], 22 V 1915, 1 VII 1940, 3 exx., leg. SS [ISEA], 17 V and 12 VII 1918, 7 exx.. leg. EM [MIZ]; Kraków-Pychowice [DA14], 10 V 1934, 1 ex., leg. EM [MIZ], 8 VI 1937. 4 exx., leg. SP [ISEA]: Kraków-Podgórki [DA14], 11 VII 1954. 1 ex., leg. WS [ISEA]; Kraków-Tyniec [DA14]. 19 VI 1932, 2 exx., leg. EM [MIZ]; Kraków-Przegorzaly [DA14], 2 VIII 1917. 2 exx., leg. EM [MIZ]: Jerzmanowice [DA16], 19 VI 1990, 1 ex., leg. JP [ISEA]; Kraków-Prądnik Biały [DA24]; 24 V 1918, 5 exx.. leg. EM [MIZ]; Kraków-Krzemionki [DA24], 24 V 1878, 1 ex., leg. SS, ok. 1900, 1 ex., leg. MR [ISEA]. 22 V 1918, 6 exx.. leg. EM [MIZ].

Malopolska Upland: Pabianice [CC82], 16-22 VI 1912, 2 exx.. leg. EI [MIZ]; Szczepanowice [DA37]. 17 VI 1994 and 6 VI 1995. 2 exx.. leg. MM [ISEA]: Pińczów [DA69]. 5 VII 1950. 2 exx., leg. JF [MIZ], 11 VII 1996, 2 exx.. leg. TW [ISEA]: Skowronno nat. res. [DA69], 2 VII 1951, 3 exx., leg. JF [MIZ]. 8 VII 1993, 1 ex., leg. MM [ISEA], 20 VI 1995, 1 ex.., leg. WZ [USMB]: Szaniec [DA79], 26 V 1993, 1 ex., leg. MM [ISEA]: Malkowice [DA76], 14 V 1991. 1 ex., leg. MM [ISEA]; Pasturka [DA79] ad Pińczów, 13 VII 1996, 1 ex., leg. RD. 19 VI 1997. 1 ex., leg. WZ [USMB]: Busko-Zdrój [DA89]. 27 VI 1950, 1 ex., leg. JF [MIZ]: Kików [DA98]. 6 VI-2 VII 1991, 5 exx., leg. JM \& MM [ISEA], 19 VI 1995, 2 exx.. leg. RD \& WZ [USMB]: Sandomierz [EB51], 13 VII 1950, 1 ex.. leg. MZ [MIZ]; Góry Pieprzowe [EB51], 9 VI 1951. 1 VI 1952, 20 V 1953, 4 exx.. leg. WS. 8 VI 2004. 1 ex., leg. DK [ISEA]: Dwikozy [EB52] ad Sandomierz. 7 VII 1967, 1 ex.. leg. WS [ISEA]; Maruszów [EB53] ad Annopol, 2 VI 1985. 1 ex., leg. JP [ISEA].

Świętokrzyskie Mts.: Zelejowa Góra [DB53] ad Chęciny, 21 VI 1967, 1 ex., leg. WS [ISEA]: Milechowy nat. res. [DB53]. 11 VI 1984. 1 ex.. leg. WJ [MIZ]: Dyminy [DB73] ad Kielce. 27 V 1952. 2 exx., leg. JF [MIZ].

Lubelska Upland: Opole Lubelskie [EB66], 28 V 1915, 1 ex., leg. EI [MIZ]; Puławy [EB69]. 15 VI 1984. 4 exx.. leg. DI [USMB]: Zaklików [EB72]. 19 IV 1950. 1 ex.. coll. MIZ: Końskowola [EB79]. 20 VIII 1928, 1 ex., leg. SU [MIZ]; Nasutów [FB09] ad Lublin. 29 V 2003. 
1 ex., leg. MM [ISEA]; Tyszowce [FB91] ad Hrubieszów, 30 VI 1985, 3 exx., leg. MM [ISEA]; Gródek [GB03] ad Hrubieszów. 10 VI 1970, 4-7 VI 1971, 24 V 1987, 6 exx.. leg. ZS. MO \& MM [ISEA].

Roztocze Upland: Ulów [FA58], 19 VII 1912, 10 leg. TE [MIZ]; Zwierzyniec [FB30], 30 VI 1985, 1 ex.. leg. DK [ISEA]; Florianka [FB30], 1 VIII 1916, 10 exx., leg. FF (1 ex. determined as Pedinus helopioides Arr.!) [MIZ]; f. distr. Grele [FB40]. 27 VII 1912. 3 exx.. leg. TE [MIZ]; Dziewcza Góra [FB41] ad Niedzieliska, 9 VI 1989, 1 ex.. leg. JP [ISEA]; Krasnobród [FB50]. 20 VIII 1912, 1 ex., leg. TE [MIZ].

Sandomierska Lowland: Szarów [DA43] ad Niepołomice, 3 VI 1984. 2 exx.. leg. TW [ISEA]; Niepolomice [DA44], VI 1912 and 14 IX 1913, 2 exx., leg. EM [MIZ]; Klaj [DA53]. 1904, 1 ex., leg. MR [ISEA]: Grodkowice [DA53] ad Bochnia, 23 VI 1935, 1 ex., leg. SP [ISEA]: Padew Narodowa [EA38]. 14 VI 1931. 10 exx.. leg. SS [ISEA]: Horyniec [FA66]. 1-30 VI 1888. 1-31 V and 1-8 VI 1889, 19 exx., leg. SS [ISEA]; „Puszcza Niepolomicka Forest”, 6 VI 1912. 1 ex., leg. TE [MIZ].

Western Beskidy Mts.: Sucha Beskidzka [CA90]. 19 $9^{\text {th }}$ century, 1 ex.. leg. AW [ISEA]; Kraków-Borek Falęcki [DA23], 31 V 1874, 2 exx., leg. BK, 22 VI-23 IX 1931, 3 exx., leg. SS [ISEA]; Łapanów [DA42], date missing. 3 exx., leg. EM [MIZ]; Rytro [DV78]. 4 VII 1892. 1 ex., leg. SS [ISEA]: Muszyna [DV96]. 19 VII 1984. 1 ex.. leg. AM [ISEA].

Eastern Beskidy Mts.: Przemyśl: Winna Góra [FA21], 26 V 1989, 1 ex., leg. MM [ISEA].

A species widespread all over Europe, reaching also Caucasus, Siberia and Mongolia. Very frequently captured in Poland in various KFP regions. In KFP, not reported from Sudety Mountains, Carpathians and the north-eastern part of Poland. There are many specimens of this species in the reviewed collections, also coming from three additional KFP regions (Masurian Lake District, Podlasie Lowland, Western Beskidy Mountains), which confirms that it is probably spread all over Poland, with the exception of high mountain areas. In MIZ collection, there are numerous voucher specimens for faunistic reports signed B. BuRAKOwsKi*, and a specimen from the collection of S. Tenenbaum (Fejfer leg.) misidentified as Pedinus helopioides Ahr.

\title{
Myrmechixenini JacqueLin du VAL, 1858
}

\section{Myrmechixenus subterraneus Chevrolat, 1835}

\author{
Pomeranian Lake District: Koszalin (Coeslin) [WA70], date missing, 2 exx., leg. AL \\ [MIZ].
}

Wielkopolska-Kujawy Lowland: f. div. Babki [XT39], 31 VII 1980. 1 ex., leg. JW [ISEA]: f. div. Goraj [XU05], 2 III 1981, 1 ex., leg. JW [ISEA].

Upper Silesia: Chelmek [CA75], 1-4 VIII 1882, 21 exx., leg. SS [ISEA].

Mazowiecka Lowland: Podkowa Leśna [DC87]. 24 IX-1 X 1934. 102 exx.. leg. TE [MIZ]; Świder [EC17], 4 VII 1903, 6 exx., leg. WM [USMB]; Dębe Wielkie [EC38]. 20 IV-7 VIII 1916. 202 exx., leg. TE [MIZ]: Siennica [EC47]. 18 XII 1933, 5 exx.., leg. AB [MIZ].

Kraków-Wieluń Upland: Kraków-Wola Justowska [DA14]. 19 V 1878. 1 ex.. leg. SS [ISEA].

Lubelska Upland: Kazimierz Dolny [EB68], IX 1934, 1 ex., leg. TE [MIZ]. 
Sandomierska Lowland: Kłaj [DA53]. 18 III 1897, 1 ex., leg. SS [ISEA].

Western Beskidy Mts.: Jastrzębia [DA12], 24-28 IV 1897. 8 exx., leg. SS [ISEA]; Glogoczów [DA12]. 19 IV 1898. 3 exx.. leg. SS [ISEA].

A species distributed everywhere in Europe, especially in its northern and central part, also recorded from Siberia. Myrmecophilous species, found in nests of Formica pratensis RETzius. It probably inhabits all Poland except of high mountain areas. KFP records presence of $M$. subterraneus in most of KFP regions, basing however on publications coming from the turn of the $19^{\text {th }}$ and the $20^{\text {th }}$ centuries. Recent reports confirm its occurrence in Poland (Jaloszyński et al. 2006; Ruta 2007). The reviewed collections contain specimens captured in three KFP regions (Pomeranian Lake District, Lubelska Upland, Sandomierska Lowland) not mentioned in KFP.

\section{Hypophlaeini Billberg, 1820}

\section{Corticeus bicolor (OLIVIER, 1790)}

Pomeranian Lake District: Gruczno [CE21]. 28 V 1996, 1 ex.. leg. DK [ISEA].

Wielkopolska-Kujawy Lowland: Nowa Sól (Neusalz) [WT43], 16 V-11 X 1907, 17 exx.. coll. MIZ; Śródka [WU83] ad Chrzypsko Wielkie, 5 V 1996, 3 exx., leg. LB [USMB]; Kościelna Wieś [BC94] ad Kalisz. 2 exx.. leg. AM [ISEA]: Rajsków [CC03] ad Kalisz, 30 X 1982. 1 ex.. leg. AM [ISEA]; Brzeziny [CC12] ad Kalisz. 15 IV 1984. 3 exx.. leg. AM [ISEA]; Łęczyca [CC76], 4 IV 1935 and 16 IV 1937, 3 exx., leg. AB [MIZ]; Sycyn Dolny [XU13], 24 IV 1994. 1 ex., leg. MA [ISEA]; Długa Goślina [XU33] ad Oborniki, 3 II 1968. 1 ex.. leg. JR [ISEA].

Mazowiecka Lowland: Otwock Wielki [DC46]. 11 VI 1950. 1 ex.. leg. BB [MIZ]; Szczytno [DC58] ad Sochaczew, 13 VII 1955, 1 ex.. leg. BB [MIZ]; Zaborów [DC79]. 14 XII 1952. 1 ex., leg. BB [MIZ]: Warszawa-Wierzbno [DC98], 20 IV 1922, 1 ex., leg. HC [MIZ]: Warszawa-Wola [DC98], cemetery, 23 VII 1930, 1 ex., leg. BB [MIZ]; Warszawa-Slużewiec [DC98], 8 VII 1908, 4 exx.. leg. TE [MIZ]; Warszawa-Mlociny [DC99], 30 XI 1947. 8 XII 1948. 28 XI 1952, 7 exx.. leg. RB. 24 II and 28 XI 1952. 3 exx., leg. BB [MIZ]: WarszawaBielany [DC99]. 24 IV and 12 V 1889. 5 exx.. leg. WM [USMB]. 8 X 1930. 23 III 1935. 23 III 1936, 23 III 1937, 1 XI 1937, 23 III 1938, 5 VII 1939, 11 exx.. leg. TE, 30 IV 1939, 3 exx.. leg. HC, 5 VII 1948, 1 ex.. leg. PD, 22 I 1950, l ex., leg. ZW, 23 I and 24 IV 1953, 2 exx.. leg. RB, 30 III 1953, 1 ex., leg. ME. 13 V- 29 VI 1950, 18 II 1959, 26 III 1961, 12 X 1962. 20 III 1965. 6 IV 1970, 20 exx., leg. BB [MIZ]; Jabłonna [DD90]. 17 VI 1907, 1 ex., leg. TE [MIZ]; Warszawa-Natolin [EC07], $16 \mathrm{~V}$ 1889. 1 ex.. leg. WM [USMB]. 11-13 IV 1930, 6 exx., leg. TE [MIZ]: Warszawa-Morysinek [EC07]. 15 XI 1953 and 11 IV 1955. 5 exx.. leg. BB [MIZ]: Warszawa-Łazienki [EC08], 21 III 1922, 1 ex., leg. HC [MIZ]; Warszawa-Goclaw [EC08]. 7 III 1948, 4 exx., leg. ZW [MIZ]: Warszawa: Zoological Garden [EC08], 18 IX 1937. 2 exx., leg. TE [MIZ]: Warszawa-Zacisze [EC09]. $19 \mathrm{~V}$ 1928. 5 exx., leg. TE [MIZ]: Obory [EC16]. 19 VIII 1950, 1 ex., leg. BB [MIZ]; Świder [EC17], 15 VII 1900 and 11 VII 1903, 2 exx., leg. WM [USMB]; Otwock [EC17], 26 IV 1915, 1 ex., leg. TE [MIZ]; Stary Otwock [EC17], 13 VI 1928, 4 exx.. leg. TE [MIZ]: Trąbki [EC45]. 6 VI 1902. 1 ex.. leg. WM [USMB]: Mienia [EC47], 14 V 1933, 1 ex., leg. AB [MIZ]; „Warszawa”, 21 V 1891 and 10 VI 1902, 3 exx., leg. WM [USMB], 16 VI and 27 VII 1948, 2 exx., leg. JF [MIZ]. 
Podlasie Lowland: Międzyrzec Podlaski [FC26], date missing, 1 ex., leg. EI [MIZ]; Białystok [FD48], 26 III 1951. 1 ex.. leg. BB [MIZ].

Bialowieża Primeval Forest: Bialowieża [FD94], Park Palacowy, 26-27 III 1949, 3 exx.. leg. ZW [MIZ].

Lower Silesia: Żarów (Saarau) [XS04], 1933, 2 exx., coll. USMB; Wroclaw: Park Szczytnicki [XS46]. 30 IV 1961. 4 exx.. leg. BB [MIZ]: Wroclaw: Park Wschodni [XS46]. 26 VI 1975. 1 ex., leg. BB [MIZ].

Trzebnickie Hills: Trzebnica [XS49], 24 XII 1975, 1 ex., leg. Л [USMB].

Upper Silesia: Katowice-Murcki (Emmanuelssegen) [CA56], 30 XI 1929 and 9 XI 1935. 2 exx., leg. KI [USMB], 30 III 1962. 5 exx., leg. BB [MIZ]; Bytom (Beuthen) [CA58], 4 IX 1931 and $5 \mathrm{~V}$ 1941, 8 exx., leg. KI [USMB].

Kraków-Wieluń Upland: Kajasówka nat. res. [DA04] ad Kaszów. 28 I 1989. 1 ex.. leg. JM [ISEA]; Kraków-Zwierzyniec [DA14], 22 IV 1900, 3 exx.. leg. MR. 8 VI 1936, 1 ex.. leg. SP [ISEA]: Kraków-Dębniki [DA14], 10 VI 1919, 14 exx., leg. SS [ISEA]: 25 XII 1983, 3 exx., leg. JM [ISEA]; Kraków-Tyniec [DA14]. 8 IV 1989, 4 exx.. leg. DK [ISEA]; Kraków-Bronowice [DA24], 11 IV 1933, 1 ex., leg. EM [MIZ]; Kraków [DA24]. Park Strzelecki, 20 X 1936, 1 ex., leg. EM [MIZ]: Kraków: Botanical Garden [DA24]. 20 V 1936, 3 exx., leg. EM [MIZ], 10 V6 XI 1936. 1 IV-23 VI 1937. 18 IX 1939, 119 exx., leg. SP, 30 III 1986, 2 exx.. leg. JM [ISEA]: Kraków: Blonia [DA24], 2 II 1938, 1 ex.. leg. SP [ISEA]; Kraków-Cichy Kącik [DA24], 11 XII 1938, 1 ex., leg. EM [MIZ]; Kraków: Park Jordana [DA24], 30 XII 1997, 1 ex.. leg. TW [ISEA]: Kraków: Planty [DA24]. 20-22 III 1974. 2 exx., leg. MG [ISEA]: Kraków [DA24]. 29 Listopada Ave., 23 IV 1985, 10 exx., leg. DK [ISEA]; Kraków-Ugorek [DA24], 10 IV 1992, 1 ex., leg. JH [ISEA]: Kraków-Plaszów [DA24], 29 XI 1916 and 29 III 1936, 2 exx., leg. EM [MIZ]; KrakówPrądnik Biały [DA24], 21 III 1935 and 18 VI 1941, 4 exx.. leg. EM [MIZ]; Kraków-Prądnik Czerwony [DA24], 1 XII 1935. 3 exx., leg. EM [MIZ].

Malopolska Upland: Ruda Pabianicka [CC82], 13 III 1959. 1 ex., leg. JZ [USMB]; Tunel [DA28] ad Miechów, VI 1983, 1 ex., leg. JM [ISEA]; Krzyżanowice [DA68]. 29 VII 1956 and 17 VII 1959. 3 exx.. leg. BB [MIZ]; Polana Polichno nat. res. (Dębina ad Mlodzawy) [DA69]. 10 IX 1954, 1 ex., leg. BB [MIZ].

Western Beskidy Mts.: Roztoka [DA82] ad Zakliczyn. 2 V 1987. 1 ex.. leg. DK [ISEA].

Bieszczady Mts.: Jablonki [EV95], 3 VII 1969, 2 exx., leg. TN [USMB]: Wetlina [FV04]. 6-8 IX 1963, 10 exx., leg. SZ [ISEA].

A species widespread everywhere in Europe, probably occurring in all Poland. Predatory larvae feed in bark beetle galleries, mainly of the European elm bark beetle - Scolytus multistriatus MARSH. and the large elm bark beetle - S. scolytus (FABR.). The species is rather numerous in the studied collections, often captured, and mentioned in many publications (Kubisz et al. 1998; Kubisz 2000; PAwŁowski et al. 2000; JAŁoszyŃsKi \& Konwerski 2001; Konwerski \& Przewoźny 2001; Kubisz \& Tsinkevich 2001; Byk \& Byk 2004; Gutowski et al. 2006; Bunalski et al. 2010).

\section{Corticeus bicoloroides (RoubaL, 1933)}

(Fig. 12)

Upper Silesia: Katowice-Murcki (Emmanuelssegen) [CA56]. 20 VI 1930, 3 exx.. leg. KI. V 1932, 4 exx., leg. HN [USMB]. 
The species is found in central and southern Europe. Its range extends from Poland (eastern border), through Czech Republic, Slovakia, Romania, Hungary, Austria, Switzerland and Italy, to France in the west, where it was captured in scattered locations throughout the country ( 9 departments). It inhabits old rotting wood of oak, poplar and beech trees, often accompanied by brown tree ants - Lasius brunneus (LATR.). The USMB collection includes voucher specimens for the publication by KuBISz (2000). One specimen, also voucher for that publication, is deposited in the collection of T. TRELLA held by ISEA. It bears a vague description „Przemyśl vic.”, but the relevant publication (TrelLa 1923) states that it has been collected at Turnica Mt. [FV19] in Eastern Beskid Mountains.

\section{Corticeus fasciatus (FABRICIUs, 1790)}

Pomeranian Lake District: Szczecin (Stettin) [VV62], date missing. 3 exx., coll. MIZ.

Wielkopolska-Kujawy Lowland: Krajkowo [XT38] ad Poznań, 16 IV 1993, 2 exx., leg. DK [ISEA].

Mazowiecka Lowland: Warszawa-Bielany [DC99]. 12 V 1889 and 1 VI 1897. 3 exx.. leg. WM [USMB]. 5 VII 1933 and 28 IV 1934. 7 exx.. leg. TE, 20 VI 1934, 3 exx., leg. JF, 26 VI 1935, 4 exx.. leg. EM, 26 VI 1935 and 1 IX 1938, 6 exx., leg. AB, 9 V 1947. 5 exx., leg. ZW, 13 V-29 VI and 26 XII 1950, 22 VI 1956, 19 V 1958, 11 II 1959, 3 VI-24 VII 1962, 19 exx., leg. BB [MIZ]: Warszawa-Natolin [EC07], $16 \mathrm{~V} 1889$ and 12 VI 1900, 4 exx., leg. WM [USMB]: Milosna [EC18], date missing. 1 ex.. leg. TE [MIZ]; Warszawa-Wawer [EC18], 29 VI 1895 and 15 V 1902, 3 exx.., leg. WM [USMB]; Stary Otwock [EC27]. 13 VI 1928, 3 exx., leg. TE [MIZ]: Trą̧bi [EC45], 6 V 1902, 1 ex., leg. WM [USMB]; Urle [ED41], 11 VI 1902, 3 exx., leg. WM [USMB].

Lower Silesia: Olawa [XS64]. V 1925, 1 ex., leg. PL [USMB].

Upper Silesia: Bytom (Beuthen) [CA58]. 1 VI 1941. 1 ex.. leg. KI [USMB].

Kraków-Wieluń Upland: Kraków-Tyniec [DA14], 3 III 1912, 1 ex., leg. TE [MIZ].

Roztocze Upland: Siedliska Tomaszowskie [FA87]. 2 V 1992. 2 exx., leg. DK [ISEA].

Sandomierska Lowland: Rudzica [DA44] ad Niepolomice, 15 VII 1949, 1 ex.. coll. USMB.

Western Beskidy Mts.: Rytro [DV78]. 21 VII 1902. 1 ex.. leg. WM [USMB].

Eastern Beskidy Mts.: Rokszyce [FA11]. 24 IX 1962. 1 ex., leg. BB [MIZ].

The species inhabits southern and central Europe, reported in Poland from few scattered localities in various regions of the country. The beetles are found in old trunks of broadleaved trees, especially oaks, in bark beetle galleries. The reviewed collections include voucher specimens for faunistic reports of B. BuRAKowski* and data on occurrence of the species in four KFP regions (Pomeranian Lake District, Sandomierska Lowland, Roztocze Upland, Western Beskidy Mountains) not recorded in KFP.

\section{Corticeus fraxini (KugELANN, 1794)}

Mazowiecka Lowland: Świder [EC17], 15 VII 1900, 3 exx., leg. WM [USMB]; WarszawaWawer [EC18]. 15 V 1902. 10 exx.. leg. WM [USMB]. 
Bialowieża Primeval Forest: Bialowieża [FD94]. 24 III- 28 IV 1921, 49 exx., leg. TE, 30 IX 1950, 1 ex., leg. BB [MIZ]; strict nat. res. [FD94], 2 X 2003, 1 ex., leg. JG [ISEA]; Białowieski N. P.. f. comp. 340 [FD94], 20 IV 1967. 2 exx.. leg. BB [MIZ]; Bialowieski N. P.. f. comp. 398 [FD94], 10 VI 1965, 17 exx., leg. BB [MIZ]; f. comp. 131C [FD95], 15 IV 2004, 1 ex., leg. JG [ISEA]: f. comp. 229B [FD95], 2 IX 1998, l ex., leg. KS [ISEA].

Upper Silesia: Rybnik-Paruszowiec (Paruschowitz) [CA25]. 23 VI 1911. 1 ex.. leg. SC [USMB]; Tworóg [CB30] ad Tarnowskie Góry, 7 IV 1984, 2 exx., leg. MW [ISEA].

Malopolska Upland: Modlica [CC92] ad Łódź, 12-19 V 1949, 3 exx.. leg. SL [USMB]: Spala [DC30]. 14 V 1906. 1 ex.. leg. WM [USMB].

Western Beskidy Mts.: Wlosań [DA22] ad Mogilany, 4 VII 1919, 1 ex., leg. EM [MIZ].

Eastern Beskidy Mts.: Wapowce [FA11] ad Przemyśl, 3 X 1886, 1 ex., leg. BK [ISEA].

Bieszczady Mts.: Lutowiska [FV25]. 16 X 1965, 1 ex., leg. RB [MIZ].

The species is widespread in Europe. Reported from single locations in few KFP regions, but confirmed by many currently published studies regarding the area of the Białowieża Primeval Forest, Podlasie Lowland, Bieszczady and Beskidy Mountains (Kubisz \& Szwalko 1991; Kubisz et al. 1998; Pawlowski et al. 2000; Kubisz \& Tsinkevich 2001; Kubisz 2004; Gutowski et al. 2006; Gutowski et al. 2010). It inhabits rotten wood and bark beetle galleries in coniferous and broadleaved trees. The collection of W. MAcZYÝski held by USMB includes specimens gathered in the beginning of the $20^{\text {th }}$ century in the vicinity of Warsaw (Swider). The locality seems to be probable (see the comments to Platydema dejeanii), but the present occurrence of the species in Mazowiecka Lowland (not listed in KFP) should be verified. The MIZ collection includes a specimen captured in Bialowieża (28 IV 1921, ex. coll S. Tenenbaum) designated as a neotype by FERRER and LUNDBERG (2003).

\section{Corticeus linearis (FABRICIUS, 1790)}

Mazowiecka Lowland: Milanówek [DC77]. 25 V 1907. 1 ex., leg. TE [MIZ].

Bialowieża Primeval Forest: Bialowieża [FD94], 27 III 1921, 2 exx., leg. TE. 6 IX 1927. 1 ex., leg. MN [MIZ].

Upper Silesia: Stolarzowice (Stollarzowitz) [CA48]. 24 VIII 1931. 1 ex.. leg. HN [USMB]; Bytom (Beuthen) [CA58], 4-11 IX and 4 XI 1931, 9 exx.. leg. KI \& HN [USMB].

Roztocze Upland: Florianka [FB30]. V 1910, 2 exx., 23 VII 1912, 1 ex. (identified as Corticeus suberis!), leg. FF [MIZ].

Sandomierska Lowland: Puszcza Niepolomicka Forest: Chobot [DA54], 15 III 1948, 3 exx.. coll. USMB.

Western Sudety Mts.: Maciejowiec [WS44]. 25 XI 1961, 3 exx.. leg. BB [MIZ].

Western Beskidy Mts.: Cieszyn [CA21], date missing. 1 ex.. leg. AH [MIZ]; Wisla-Glębce [CV49], 19 VIII 1961, 12 exx., leg. BB [MIZ]; Krynica-Kopciowa [DV97]. VII 1985, 28 VI and 26 VII 1989, 4 exx.. leg. DK [ISEA].

Pieniny Mts.: Male Pieniny Mts.: Jaworki [DV67], 31 VII 1928. 1 ex.. leg. TE [MIZ].

Euro-Siberian species, widespread in Europe. According to KFP, it probably inhabits whole territory of Poland except of high mountain areas, although it has not yet been reported from many KFP regions. This speculation is still valid, but not yet confirmed 
in spite of many publications (Borowiec et al. 1992; Gutowski \& KubISz 1995; KuBISZ 1995; Kubisz \& Tsinkevich 2001; Konwerski \& Przewoźny 2001; Kubisz \& Szafraniec 2003; Gutowski et al. 2005; Gutowski et al. 2006; Tykarski 2006; Bidas 2007; BuNALSKI et al. 2010; GuTOWSKi et al. 2010). The reviewed collections include voucher specimens for the reports of B. BuRakowski*, and specimens captured in Sandomierska Lowland and Western Beskidy Mountains (information not recorded in KFP). TYKaRSKI (2006) reported occurrence of that species in the Tatry Mountains. The beetles are found in bark beetle galleries (upper part of tree trunk and narrow tops of coniferous tree branches, especially of pine trees).

\section{Corticeus longulus (GYLLENHAL, 1827)}

Mazowiecka Lowland: Warszawa-Bielany [DC99], 4 V 1894, 3 V 1900, 26 X 1901. 4 exx.. leg. WM [USMB]: Świder [EC17]. 18 IX 1900. 2 exx.. leg. WM [USMB]: Warszawa-Wawer [EC18], 26 IV 1970, 3 exx., leg. BB [MIZ]; Dębe Wielkie [EC38]. 18 IV and 12 V 1916, 4 exx.. leg. TE \& EI [MIZ]: Urle [ED41]. 17 VIII 1958, 1 ex., leg. MZ [MIZ].

Bialowieża Primeval Forest: Białowieża [FD94]. 24 III 1921. 3 exx.. leg. TE [MIZ].

Upper Silesia: Bytom (Beuthen) [CA58], 1 V 1941, 1 ex., leg. KI [USMB]; Lubliniec [CB31], 1 V 1994, 4 exx., leg. RD [USMB].

Kraków-Wieluń Upland: Kraków-Wola Justowska [DA14]. 11 V 1912. 1 ex.. leg. TE [MIZ]: Kraków-Las Wolski [DA14]. 5 V 1973, 1 ex.. leg. MG [ISEA].

Malopolska Upland: Miechów [DA38], 7 IV 1912, 1 ex., leg. JF [MIZ]; Krzyżanowice [DA68], 17 VII 1959. 2 exx.. leg. BB [MIZ]; Spala [DC30], 27 IX 1900. 1 ex., leg. WM [USMB].

Sandomierska Lowland: Klaj [DA53], 21 III 1937, 1 ex., leg. EM [MIZ]; „Puszcza Niepolomicka Forest", 29 V 1951, 3 exx.. coll. USMB.

The species is widespread in the central part of Europe. According to KFP, it is known in Poland from single localities, only in three regions. Since 1987, many publications appeared, confirming a wider range of its species in our country (GRZYwOCZ \& Szoltys 1996; Kubisz \& Tsinkevich 2001; Sienkiewicz \& Konwerski 2005; Bidas 2007; Bunalski et al. 2010). The reviewed collections include specimens captured in Upper Silesia and Western Beskidy Mountains (not listed in KFP). The beetles are connected with coniferous trees, mainly with pines and spruces, found under the bark or in galleries of bark beetles).

\section{Corticeus pini (PANzer, 1799)}

Wielkopolska-Kujawy Lowland: Bobrowniki [CD65], 25 IV 1971, 1 ex.. leg. BB [MIZ].

Mazowiecka Lowland: Warszawa-Bielany [DC99]. 11 II 1959. 2 exx., leg. BB [MIZ].

Bialowieża Primeval Forest: Białowieski N. P.. f. comp. 399 [FD94]. 15 V 1965. 1 ex.. leg. BB [MIZ].

Kraków-Wieluń Upland: Kraków-Bielany [DA14], 15 VI 1900, 4 exx.. leg. MR [ISEA]; Kraków-Przegorzaly [DA14]. ca. 1900. 1 ex.. leg. MR [ISEA]; Kraków: Botanical Garden [DA24], 8 IX 1936, 1 ex.. leg. SP [ISEA]. 
Świętokrzyskie Mts.: Łysa Góra Mt. [EB03], 14 VII 1932, 2 exx., leg. TE [MIZ].

Roztocze Upland: Florianka [FB30]. 1 XI 1913, 1 ex., leg. FF [MIZ]; Krasnobród [FB50]. 27 III 1934. 1 ex.. leg. AB [MIZ].

Sandomierska Lowland: Tarnów [DA94], 29 IX 1891, 1 ex.. leg. MR [ISEA]; Rzeszów [EA74], 2 III 1876, 1 ex., leg. MR [ISEA].

A Mediterranean species, rare and sporadically found in the central Europe. According to KFP, it has been reported from few localities in only six regions of Poland. The reviewed collections include specimens captured in localities of three KFP regions (Wielkopolska-Kujawy Lowland, Mazowiecka Lowland, Sandomierska Lowland) not indicated by KFP.

\section{Corticeus suberis (LuCas, 1846)}

Upper Silesia: Bytom (Beuthen. Stadtwald) [CA58]. 14 IX 1938. 1 ex.., leg. HN [USMB].

A central-European species, relict of old-growth forests. Recorded in Poland on the basis of notes dating back 100 years. The MIZ collection includes a specimen from the collection of F. FEJFER (a voucher specimen for FEJFER 1924), identified as C. suberis, which is in fact a misidentified specimen of $C$. linearis (FABR.). The authors of KFP have express their doubt as to its occurrence in Poland. The specimen included in the USMB collection is an evidence that the species has nevertheless occurred here. Although it was properly qualified to the DD category (Polish Red List, PAwlowski et al. 2002), one can consider changing its category to EX?.

\section{Corticeus suturalis (PaYkuld, 1800)}

Bialowieża Primeval Forest: Bialowieża [FD94]. 25 III 1921, 1 ex., leg. TE [MIZ]; Bialowieski N. P.. f. comp. 399 [FD94]. 26 IX 1974, 4 exx., leg. BB [MIZ]; Bialowieski N. P.. f. comp. 369 [FD94], 18 IV 1967, 3 exx., leg. BB [MIZ]: Bialowieski N. P., f. comp. 371 [FD94]. 22 IV 1968, 2 exx.., leg. BB [MIZ]; strict nat. res. [FD94]. 2 X 2003, 2 exx., leg. JG [ISEA].

A boreo-montane species, little known in Poland, reported from three KFP regions so far (KFP; Kubisz \& SzwaŁko 1991; Kubisz 1995; Gutowski et al. 2006). It inhabits trunks of dead, standing spruces or pines, which is less common (under the bark or in bark beetle galleries).

\section{Corticeus unicolor Piller \& Mitterpacher, 1783}

Pomeranian Lake District: Bielinek nad Odra [VU46], 11 VIII 1990, 10 VI 1999, 3 exx., leg. LB [ISEA]; Puszcza Bukowa. Kolowskie Parowy nat. res. [VV71]. 10 IV 1988. 4 exx.. leg. MA [ISEA]; Puszcza Bukowa, Buczynowe Wąwozy nat. res. [VV80], 26 V 1988, 5 exx., leg. LB [ISEA]: ad Koszalin [WA70], 5 V 1978, 1 ex., leg. BB [MIZ].

Wielkopolska-Kujawy Lowland: Goluchów [YT05] ad Kalisz, 17 III 1981. 1 ex.. leg. AM [ISEA]: Brzeziny [CC12] ad Kalisz, 15 IV 1984, 6 exx., leg. AM [ISEA]. 
Mazowiecka Lowland: Żbików [DC88], 5 X 1958, 2 exx., leg. BB [MIZ]; Warszawa-Bielany [DC99], 28 IV 1934, 3 exx., leg. TE, 13-24 V 1950. 6 VI 1966, 6 IV 1970, 1 I 1972. 10 exx.. leg. BB. 19 VI 1973, 2 exx., leg. EN [MIZ]. 2 V 2005, 1 ex.. leg. EN [ISEA]: Warszawa-Natolin [EC07], 24 V 1893, 2 exx., leg. WM [USMB]; Warszawa-Wawer [EC18], 8 VI 1898, 1 ex., leg. WM [USMB]; ,Warszawa”, 10 VI 1892, 2 exx., leg. WM [USMB].

Bialowieża Primeval Forest: ad Hajduki [FD84]. 2 VI 1993. 1 ex.. leg. JG [ISEA]; Gruszki [FD85], 28 VII 1986, 3 exx., leg. DK [ISEA]; Bialowieża [FD94], 29 IX 1950, 1 ex., leg. BB [MIZ]; f. comp. 317 [FD94], 20 IV 1967. 1 ex., leg. BB [MIZ]; f. comp. 340 [FD94]. 6 IV 1955. 3 exx., leg. BB [MIZ]: f. comp. 369 [FD94]. 28 V 1999. 2 exx.. leg. ST [ISEA]: f. comp. 370 [FD94], 22 IV 1968, 1 ex., leg. BB [MIZ]; Kosy Most [FD95], 28 VII 1986, 2 exx., leg. DK [ISEA]: f. comp. 223 [FD95], $28 \mathrm{~V}$ 1954, 2 exx., leg. BB [MIZ]; f. comp. 285 [FD95], $10 \mathrm{~V}$ 1959. 2 exx.. leg. BB [MIZ]: f. comp. 286D [FD95]. 19 VII 1995. 1 ex.. leg. LB [ISEA].

Lower Silesia: Buczyna Szprotawska nat. res. [WT50] ad Przemków, 19 V 2007, 9 exx.. leg. RD [USMB].

Upper Silesia: Rybnik-Paruszowiec (Paruschowitz) [CA25]. 25 VIII 1909. 2 exx.. leg. SC [USMB]; Segiet nat. res. [CA48], 29 VI 1995, 3 exx.. leg. WZ [USMB]; Katowice-Murcki (Emmanuelssegen) [CA56]. 9 VI 1912 and 15 VI 1913, 6 exx.. leg. GP, 13 XI 1928, 20 VI 1930, 25 III and 17 V 1931. VIII 1942. 15 exx.. leg. KI [USMB]; Katowice: Las Murckowski nat. res. [CA56], 20 VIII 1996, 1 ex., leg. ST [ISEA]: Bytom (Beuthen) [CA58], 7 X 1925 and 9 X 1934. 2 exx.. leg. KI, 21 XI 1938, 1 ex., leg. HN [USMB]; Zawadzkie [CB20], 18 IV 1930, 1 ex., leg. KI [USMB]: Tworóg [CB30] ad Tarnowskie Góry, 1 X 1983. 7 IV 1984. 2 exx.. leg. ST \& MW [ISEA]: Lubliniec [CB31], 28 VI 1994, 4 exx., leg. RD [USMB].

Kraków-Wieluń Upland: Zloty Potok [CB91] ad Częstochowa, 11 V 1902, 1 ex.. leg. SS [ISEA].

Malopolska Upland: Spala [DC41]. 27 IX 1900, 2 exx., leg. WM [USMB].

Świętokrzyskie Mts.: Huta Szklana [DB93], 10 V 2002, 2 exx.. leg. DK [ISEA]; Łysa Góra Mt. [EB03], 2 VI 1956, 1 ex.. leg. BB [MIZ].

Roztocze Upland: Czartowe Pole nat. res. [FA49] ad Hamernia. 17 VIII 1982 and $10 \mathrm{~V}$ 1988, 4 exx.., leg. DI [ISEA. USMB]; Panasówka [FB30], 20 VIII 1911. 6 exx.., leg. TE [MIZ]; Florianka [FB30]. 2 VIII 1911 and 25 VII 1913, 6 exx.. leg. FF [MIZ]: f. distr. Grele [FB40]. 25 VII 1910 and 27 VII 1912, 21 exx. leg. TE [MIZ]: Katy-Kosobudy [FB41], 21 VIII 1912, 2 exx.. leg. FF [MIZ]; Obrocz [FB41], 30 VII 1912, 3 exx.. leg. FF [MIZ]: Krasnobród [FB50], 30 III 1934, 11 exx., leg. AB [MIZ]; Nart nat. res. [FB50], 15 VII 1910 and 22 VIII 1912, 21 exx., leg. TE [MIZ]. VIII 1981, 1 ex., leg. TW, 9 VII 1999. 3 exx., leg. ST [ISEA].

Western Beskidy Mts.: Czantoria Mt. [CA40]. 1880, VII-VIII 1884, 21 VIII 1886, VIIVIII 1887. 7 exx., leg. BK [ISEA], 19 VI-11 VII 1962, 3 exx.. leg. BB [MIZ]: Stożek Wielki Mt. [CA40], 26 IX 1898, 1 ex., leg. BK [ISEA]: Równica Mt. [CA40], 5 VIII 1873. VIII 1884. 5 exx., leg. BK [ISEA]; ad Szczyrk [CA50]. VIII 1884, 1 ex., leg. BK [ISEA]; Skrzyczne Mt. [CA50] ad Żywiec. VIII 1884, 1 ex.. leg. BK [ISEA]: Barania Góra Mt. [CV59]. 30 VI 1955. 1 ex., leg. BB [MIZ]: Tymbark [DA50]. 3 VI 1905, 1 ex., leg. SS [ISEA]; Młodów [DV77] ad Rytro, 12 V 1892, 15 VII 1898, 12 V 1899, 88 exx., leg. SS [ISEA]; Rytro [DV78], 2 V-16 VIII 1892, 14 VII 1894, 9-21 VI 1896, 30 VII-5 VIII 1898, 11 V-7 VIII 1899, 10 VI 1900, 1 VI-5 VII 1901. 10 VI-21 VII 1902. 23 VII 1903. 85 exx., leg. SS [ISEA]. 10 VII-10 VIII 1929. 68 exx.. leg. TE. 14 VII 1949, 18 exx., leg. MZ [MIZ]: Beskid Sądecki Mts.: Pusta Wielka [DV87]. 5 IX 1987, 1 ex., leg. AM [ISEA].

Eastern Beskidy Mts.: Barwinek [EV57]. 25 VII 1988. 1 ex.. leg. DK [ISEA]; Sanok [EV89]. 26 V 1952, 5 exx., leg. RB [MIZ]; Panieński Czub [FA00] ad Bircza, 22 V 1879, 4 exx.. 
leg. BK [ISEA]: Góra Biala (Splawa) [FA10], 7 VI-6 VII 1884, 7 exx., leg. BK [ISEA]: Holubla [FA11]. 25 V-29 VI 1884. VI 1886, 11 exx., leg. BK [ISEA]: Wapowce [FA11] ad Przemyśl. 3 X 1889, 4 exx., leg. BK [ISEA]; Kaczmarowa Mt. [FA21] ad Łętownia. 28 IV 1879. 3 exx.. leg. BK [ISEA]; Przemyśl: Pralkowce [FA21], 27 IV-31 V 1879, 8 V 1883, 18 V 1884, 13 exx.. leg. BK [ISEA]; Przemyśl: Ostrów [FA21]. 25 V 1882, 1 ex., leg. BK [ISEA]; Przemyśl: Winna Góra [FA21]. 1880. 1 ex.. leg. BK [ISEA]; Kamienna Laworta Mt. [FV17] ad Ustrzyki Dolne. 19 VI 1880, 4 exx., leg. BK [ISEA]; Jaworniki Mt. ad Bandrów Narodowy [FV27]. 21 VI 1881. 1 ex., leg. BK [ISEA].

Bieszczady Mts.: Wetlina [FV04]. 7-8 IX 1963, 8 exx.. leg. SZ [ISEA]; Bukowiec [FV06] ad Solina, 14 IX 1969, 1 ex., leg. SZ [ISEA]; Otryt Mt. [FV15], 16 VI 1958 and 6 VI 1970. 6 exx., leg. BB [MIZ].

Pieniny Mts.: Krościenko [DV57]. 27 VII 1949. 16 exx., leg. RB [MIZ]; Droga Pienińska [DV57], 17 VII 1939. 3 exx., leg. TE [MIZ]; Ociemne [DV57]. 14 VIII 1939. 2 exx., leg. TE [MIZ]; Pustelnia św. Kingi [DV57], 1 VI 1929, 1 ex.. leg. TE [MIZ].

Tatry Mts.: Hala Gąsienicowa [DV25]. 8 VII 1897. 1 ex.. leg. SS [ISEA].

A European species, widely distributed. According to KFP, it probably occurs in whole Poland, although not yet reported from some regions. It is the most often captured species of Corticeus PILL. \& MrTt. genus, which is reflected by its numerous representation in the surveyed collections and reports in scientific publications (KuBISz et al. 2000; Pawlowski et al. 2000; Kubisz \& Tsinkevich 2001; Klejdysz \& Kubisz 2003; Byk \& Byk 2004; Kubisz 2004; Gutowski et al. 2006; Bunalski et al. 2010). The ISEA collection includes a specimen captured in the Tatry Mountains (a region not listed in KFP); in MIZ collection, there are numerous voucher specimens for the reports by B. BurakowsKi*.

\section{Scaphidemini ReITTER, 1922}

\section{Scaphidema metallicum metallicum (FABRIcIUs, 1792)}

Pomeranian Lake District: Bielinek nad Odra [VU46], 25 VI 1983, 1 ex., leg. LB [ISEA]; 31 V-3 VI 1983, 3 exx., leg. DI [USMB]: Ślesin [XU79], 21 V 1995, 1 ex.. leg. MM [ISEA]; Rudzki Most [XV93]. 12 V 1978, 1 ex., leg. JG [MIZ]; Tleń [CE14], 27 VII 1922. 1 ex.. leg. Schuster [MIZ].

Wielkopolska-Kujawy Lowland: Laski Lubuskie [VU71] ad Kostrzyn. 9 VI 2003, 1 ex.. leg. MM [ISEA]; Brzeziny [CC12] ad Kalisz, 15 IV 1984, 1 ex., leg. AM [ISEA]; Łęczyca [CC76]. 25 VIII 1934, 1 ex., leg. AB [MIZ]: Pożegowo [XT29] ad Śrem. 12 IV 1958, 2 exx.. leg. BB [MIZ]: Krajkowo [XT38], 28 VII 1984, 2 exx., leg. DI [USMB]; Rogalin [XT39], 19 III 1983, 2 exx., leg. JW [USMB]: Moja Wola [XT80] ad Kalisz, 30 XI 1987, 1 ex., leg. AM [ISEA]: Sycyn Dolny [XU13]. 15 V 1994. 1 ex.. leg. MA [ISEA]: Poznań [XU21]. 28 III 1976. 1 ex.. leg. JG [MIZ]: Poznań-Golęcin [XU21], 4 VI 1931, 4 exx.. leg. HC [MIZ].

Mazowiecka Lowland: Puszcza Kampinoska Forest: Grabowy Grunt [DC79]. 6 I 1953. 1 ex.. leg. BB [MIZ]; Puszcza Kampinoska Forest: Przykop [DC79]. 1 III 1953, 1 ex.. leg. BB [MIZ]; Puszcza Kampinoska Forest: Zaborów [DC79]. 12 IV 1953 and 21 III 1965, 2 exx., leg. BB [MIZ]: Żbików [DC88], 29 IV 1951, 1 ex., leg. RB, 5 X 1958, 2 exx., leg. BB [MIZ]: 
Kampinoski N. P., Lipków [DC89]. 2 IV2004, 1 ex., leg. WJ [ISEA]: Lomianki [DC99], 17 III 1923. 1 ex.. leg. HC [MIZ]; Warszawa-Mlociny [DC99], 10 II 1952. 2 exx.. leg. BB [MIZ]; WarszawaBielany [DC99]. 29 IV 1934, 3 exx., leg. TE, 5 VII and 26 XII 1950. 3 I 1954. 9 X 1955, 20 II 1959. 19 III 1967, 28 IV 1970, 7 exx., leg. BB [MIZ]; Konstancin-Jeziorna [EC07], 27 IV 1933. 1 ex., leg. JF, 6 V-8 VI 1934, 2 exx.. leg. AB [MIZ]; Warszawa-Morysinek [EC07], 14 I 1951. 1 ex.. leg. BB [MIZ]; Warszawa-Natolin [EC07]. 4 V 1905. 1 ex.. leg. WM [USMB]; WarszawaŁazienki [EC08], 19-20 V 1905, 2 exx., leg. WM [USMB], 16 IV 1914, 2 exx.., leg. TE [MIZ]: Świder [EC17], 15 VII 1900, 1 ex., leg. WM [USMB]; Warszawa-Wygoda [EC18], 20 VI 1961. 1 ex., leg. WD [MIZ]: Zegrze [ED01]. 7 I 1951. 1 ex.. leg. BB [MIZ].

Lower Silesia: Świebodzice [WS93]. 20 IV 1953, 1 ex.. leg. RB [MIZ]; Żarów (Saarau) [XS04], VII 1941, 1 ex., coll. USMB; Wroclaw [XS46], 6 III 1966, 4 exx., leg. TN [USMB]: Łanów (Königswalde) [XS60]. 1 X 1929. 12 exx.. leg. KI [USMB]; Grodków (Grottkau) [XS61]. 1 X 1929, 9 exx.. leg. KI [USMB].

Trzebnickie Hills: Trzebnica [XS49], 2 IV 1976, 1 ex., leg. J [USMB].

Upper Silesia: Gliwice [CA37]. 5 VI 1998, 3 exx.. leg. ST [ISEA]; Gliwice-Łabędy (Laband) [CA38], 28 II and 2-28 IV 1926, 6 IV 1928, 5 exx., leg. KI, 4 IX 1925 and 3 V 1938. 2 exx.. leg. HN [USMB]; Rotuz nat. res. [CA42] ad Czechowice-Dziedzice, 19 X 1996, 25 VI 1997. 2 exx.. leg.PR [ISEA]: Tarnowskie Góry [CA49]. 15 IV 1985. 2 exx.. leg. MW [ISEA]: Bytom (Beuthen) [CA58]. VII 1910, 4 exx., leg. GP [USMB]; Zaborze [CA74] ad Oświęcim. 5-6 VIII 1902, 23-26 VII 1903, 5 exx.. leg. SS [ISEA]: Gromiec [CA74] ad Libiąż, 24 IV 1882. 1 ex., leg. SS [ISEA].

Kraków-Wieluń Upland: Czatkowice [DA05] ad Krzeszowice, 8 V 1882, 5 exx.. leg. SS [ISEA]: Smoleń [DA08]. 2 VI 1998, 1 ex., leg. DK [ISEA]: Kraków-Zwierzyniec [DA14]. 22 IV 1923, 1 ex.. leg. SS [ISEA]; Kraków-Salwator [DA14], IX 1985, 1 ex.. leg. JM [ISEA]: Kraków-Chelm [DA14]. VII 1965, 1 ex., leg. MG [ISEA]; Kraków-Wola Justowska [DA14]. 8 XII 1876, 12 VI 1878, 13 exx., leg. SS [ISEA]; Kraków-Panieńskie Skaly [DA14], 22 III 1936. 1 ex., leg. EM [MIZ]: Kraków-Sikornik [DA14], 6 IX 1884, 1 ex., leg. SS, 10 V 1984. 19 IV 1987, 5 exx., leg. DK [ISEA]: Kraków-Przegorzaly [DA14], 7 V 1917, 1 ex.. leg. SS. 8 IV 1987, 4 I 1991, 3 exx., leg. JM [ISEA]: Kraków-Dębniki [DA14], ca. 1900, 6 exx.. leg. MR [ISEA]; Kraków-Zakrzówek [DA14]. 16 III 1987. 1 ex.. leg. JM [ISEA]; Kraków-Pychowice [DA14]. 9 IV 1987, 1 ex., leg. JM [ISEA]; Kraków-Kostrze [DA14]. 10 X 1920. 1 ex., leg. EM [MIZ]: Kraków: Park Jordana [DA24], 20 XI 1931, 1 ex.. leg. EM [MIZ]; Kraków-Olsza [DA24], 14 III 1911, 1 ex., leg. SP [ISEA]: Kraków: Botanical Garden [DA24], 30 IV 1936. 1 IV-7 VII 1937. 17 III-26 VI 1938, 21 VI 1939. 29 exx.. leg. SP, 30 III 1986, 1 ex., leg. JM [ISEA]; Kraków-Krowodrza [DA24], 23 III 1935, 20 exx.. leg. EM [MIZ]; Kraków-Prądnik Czerwony [DA24]. 6 VI 1936, 1 ex.. leg. EM [MIZ]: Kraków-Prądnik Biały [DA24]. 28 X 1940. 1 ex.. leg. EM [MIZ]; Kraków-Lobzów [DA24]. 19 III 1933 and 29 I 1939. 2 exx.. leg. EM [MIZ]; Krakow-Krzemionki Podgórskie [DA24], 25 V 1916, 2 exx., leg. EM [MIZ]; Kraków-Zablocie [DA24], 13 X 1923 and 9 IV 1933, 4 exx.. leg. EM [MIZ]; „Kraków”, 28 III 1914. 2 exx., leg. TE [MIZ].

Malopolska Upland: Wielki Dól [DA37] ad Miechów, 14 VI 1991, 1 ex.., leg. MM [ISEA]: Wola Chroberska [DA68], 3 XI 1992, 1 ex., leg. JP [ISEA].

Lubelska Upland: Pulawy [EB69]. 22 II 1912, 1 ex., leg. TE [MIZ].

Roztocze Upland: Nart-Obrocz nat. res. [FB50]. 30 VI 1985, 1 ex.. leg. DK [ISEA], 6 V 1988, 5 exx., leg. DI [USMB].

Sandomierska Lowland: Podlęże [DA44]. 18 III 1913. 1 ex.. leg. EM [MIZ]; Stanislawice [DA53] ad Klaj, 30 III 1954, 1 ex., leg. BB [MIZ]. 
Western Sudety Mts.: Sobieszów [WS43] ad Jelenia Góra, 19 IV 1953, 2 exx.. leg. RB [MIZ].

Western Beskidy Mts.: Cieszyn [CA21], date missing. 2 exx., leg. VW. 15 III 1952. 1 ex.. leg. WE [MIZ]; Babia Góra Mt. [CV99]. 25 VII 1938. 1 ex., leg. SP [ISEA]; Leńcze [DA02]. 11 IV 1895. 2 exx., leg. SS [ISEA]: Lanckorona [DA02], 27 XI 1938, 1 ex., leg. EM [MIZ]: Glogoczów [DA12]. 25 V 1897. 1 ex.. leg. SS [ISEA]; Paleśnica [DA81] ad Zakliczyn. 6 VI 1998, 1 ex., leg. DK [ISEA]: Mogila Mt. [DA82] ad Zakliczyn. 20 V 1999, 1 ex., leg. LB [ISEA]: Popowice [DV78] ad Stary Sącz, 21 V 1999, 1 ex., leg. LB [ISEA]; Nowy Sącz [DV79]. 18 VI 1892. 2 exx.. leg. SS [ISEA]: Brzanka Mt. [EA02] ad Gromnik. 5 VI 1998. 1 ex.. leg. DK [ISEA].

Eastern Beskidy Mts.: Cergowa Mt. [EV58] ad Dukla, 23 VIII 1966, 1 ex., leg. AS [ISEA]; Reczpol [FA11] ad Krzywcza. 3 IV 1989. 2 exx.. leg. JP [ISEA]: Hohubla [FA11]. 17 V 1879. 11 exx., leg. BK [ISEA], 3 XI 1955, 3 exx., leg. BB [MIZ]: Przemyśl: Pralkowce [FA21]. 16 VI 1881, 1 ex., leg. BK [ISEA]: Przemyśl: Wzniesienie [FA21], 12 VI 1884, 1 ex., leg. BK [ISEA]: Łuczyce [FA31]. 7 V 1990. 1 ex.. leg. MM [ISEA].

Bieszczady Mts.: Otryt Mt. [FV15]. 16 VI 1958, 1 ex., leg. BB [MIZ].

Pieniny Mts.: Krościenko [DV57]. 5 V 1974, 1 ex., leg. BB [MIZ]; Kras [DV57], 15 V 1972. 2 exx.. leg. BB [MIZ]: Biala Woda nat. res. [DV67]. 30 IV 1974. 1 ex.. leg. BB [MIZ].

A Euro-Siberian species, widespread everywhere in Europe. Apart from the a nominotypical subspecies of S. metallicum metallicum (FABR.), there is a subspecies of S. metallicum bicolor (FABRICIUS, 1798), reported from Hungary and Ukraine (LōBL \& SMETana 2008), not listed, however, in a study of Ukrainian fauna (TSHERnIEy 2005 ). According to KFP, this species probably occurs in the whole territory of Poland except from high mountain areas. This is supported by a comparatively large number of specimens deposited in the surveyed collections, inter alia, from localities in four KFP regions (Pomeranian Lake District, Roztocze Upland, Pieniny Mts., Bieszczady Mts.) not listed in KFP. The MIZ collection includes voucher specimens for notes signed B. BURAKowsKI* .

\section{Conclusions}

The present study on beetles of the Tenebrionidae family contains data on more than 10,500 specimens belonging to 78 species deposited in three largest entomological collections in Poland (ISEA, MIZ and USMB).

The oldest specimen of darkling beetles preserved in the studied collections belongs to Isomira murina murina (LinNAEUS, 1758), and was collected by A. WAGA in 1859 (Kielce, Świętokrzyskie Mts.). It may be assumed that the data from presented collections include the last 150 years.

The review of the above listed materials allowed to confirm identification of specimens belonging to 14 doubtful species or important for interpretation of their occurrence in Poland: Blaps halophila FiscH., Oodescelis melas (FISCH.), Bolitophagus interruptus ILL., Eledonoprius armatus (PAnz.), Platydema dejeanii (CAST. \& BR.), Alphitophagus bifasciatus ( $\mathrm{SAY}$ ), Alphitobius laevigatus (FABR.), Tenebrio opacus (DuFt.), Laena reitteri WEISE, Allecula rhenana BACH, Hymenophorus doublieri Muls., Cteniopus 
sulphuripes (GERM.) and not counted into Polish fauna by KFP (listed without index numbers) Gonocephalum gramulatum pusillum (FABR.) and Latheticus oryzae WATER.

The reviewed collections include specimens representing 14 species listed in Polish Red Book of Endangered Animals (Konwerski 2004) and in Polish Red List (Pawıowski et al. 2002): Blaps halophila Fisch., B. lethifera MARSH., B. mortisaga (L.), B. mucronata Latr., Oodescelis melas (FIsch.), Hymenophorus doublieri Muls., Cteniopus sulphuripes (GERm.), Podonta nigrita (FABr.), Bolitophagus interruptus ILl., Eledonoprius armatus (PANZ.), Neomida haemorrhoidalis (FABr.), Corticeus suberis (LuCAS), Tenebrio opacus DuFT. and Laena reitteri WeISE; however, the specimens of: Gnaptor spinimanus (PAL.), Bius thoracicus (FABR.), Mycetocharis obscura (ZеITt.), M roubali MARAN and Menephilus cylindricus (HERBST), which had been captured in Poland, were not found. Treating Isomira murina murina (L.) as an older synonym of I. semiflava (Küst.) causes that this name cannot be regarded a separate species. The data on occurrence of I. murina murina in Poland do not give a reason to treat this species as endangered, and so to include it in Polish Red List (PAWŁowski et al. 2002).

The following cases of misidentification were found:

- a voucher specimen (deposited in the MIZ collection) used in publications by Tenebaum $(1918,1923)$ and secondarily cited by KFP; a specimen captured by F. FEJFER (Roztocze Upland, Florianka) belongs in fact to Crypticus quisquilius (L.), and not to Pedinus helopioides AHr.;

- a voucher specimens (deposited in the ISEA collection) used in the publication by Szymczakowski (1973) and secondarily cited by KFP; the specimens captured in the Radomyśl in fact belongs to Oodescelis melas (Fisch.) and not Oodescelis polita (STURM);

- a voucher specimen (deposited in the ISEA collection) used in the publication by SZYMCZAKOWSKI (1973) and secondarily cited by KFP; the specimen captured by that collector in the vicinity of Ojców in fact belong to Isomira murina (L.) and not Isomira icteropa (KÜST.), thus the latter one should be deleted from the list of species occurring in Poland;

- a voucher specimen (deposited in the MIZ collection) used in KFP, and cited as B. Burakowski( ${ }^{*}$ ); specimens captured by B. Burakowski (Lomianki) belongs in fact to Palorus subdepressus Woll. and not Palorus ratzeburgii (WISSM.);

- a voucher specimen (deposited in the MIZ collection) used for publication by FEJFER (1924) and secondarily cited by KFP; the specimen collected by F. FEJFER (Roztocze Upland, Florianka) belongs actually to Corticeus linearis (FABR.), and not Corticeus suberis (LuCAs).

In case of 19 species, the authors failed to find, during the review of the collections, specimens captured in Poland which could document records published in old works and in KFP: Gnaptor spinimanus (PAL.), Myrmechixenus vaporariorum GuĖ., Bius thoracicus (FABR.), Menephilus cylindricus (HeRBST), Mycetocharis roubali MARAN, Omophlus lividipes MuLs. and not counted into Polish fauna by KFP (listed without index numbers) Tentyria nomas (PAL.), Asida sabulosa (Fues.), Prosodes obtusa (Fabr.), Phaleria cadaverina (FABr.), Pentaphyllus chrysomeloides (Rossi), Myrmechixenus 
picinus (AuBE), Corticeus versipellis (BAUDI), Upis ceramboides (L.), Accanthopus velikensis PllL. \& Mitt. [=Enoplopus dentipes (Rossi)], Odocnemis badius (REDt.), Mycetocharis obscura (ZEITT.), Omophlus lepturoides (FABR.) and O. picipes (FABR.).

For more than a half of the studied species (44), data on their occurrence in faunistic regions not listed in KFP.

Information regarding capturing some species, not reported from Poland in KFP, was published for the first time. This applies to: Centorus elongatus (HERBST), Cryphaeus cornutus (FISH.) (voucher specimen for the record by STEBNICKA 1991) and Nalassus convexus (Сом.).

\section{ACKNOWLEDGMENTS}

We would like to express our gratitude to the director of the Department of Nature of the Upper Silesian Museum in Bytom, Roland Doвosz, for granting access to the collection, and to Marcin KAMIŃsKI and Marcin RAs for technical assistance during works on the project. The authors are thankful to Roland Dobosz, Łukasz Przybylowicz and Dawid Schimrosczyk for taking the photographs.

\section{REFERENCES}

BDAS M. 2007. Nowe stanowiska czarnuchowatych (Coleoptera: Tenebrionidae) w Górach Świętokrzyskich. Wiadomości Entomologiczne 26 (4): 291-292.

BorowIEC L. 1991. Nowe i rzadkie dla Polski gatunki chrząszczy (Coleoptera). Wiadomości Entomologiczne 10 (4): 197-205.

BoRowec L. 1992. The leaf-beetles in the collection of Wojciech MĄCZÝ́sKI (1860-1911) (Coleoptera: Chrysomelidae). Annals of the Upper Silesian Museum in Bytom, Natural History 3: 3-29.

Borowete L.. Kanta J. \& Wanat M. 1992. Chrzaszcze (Coleoptera) nowe dla Puszczy Bialowieskiej. Wiadomości Entomologiczne 11 (3): 133-141.

Bouchard P.. Lawrence J. F.. Davies A. E. \& Newton A. F. 2005. Synoptic classification of the world Tenebrionidae (Insecta: Coleoptera) with a rewiev of family-group names. Annales Zoologici 55 (4): 499-530.

Buntalski M., Konwerski S., Przewoźny M. \& Ruta R. 2010. Nowe dane o rozmieszczeniu chrzaszczy z rodziny czarnuchowatych (Coleoptera: Tenebrionidae) na Nizinie Wielkopolsko-Kujawskiej. Część 2: Lagriinae i Diaperinae. Wiadomości Entomologiczne 29 (2): 75-86.

Burakowski B. 1976. Rozmiazgowate - Pythidae. Omiękowate - Lagriidae. Cisawkowate - Alleculidae. Klucze do oznaczania owadów Polski, cz. XIX Chrząszcze - Coleoptera, z. 88-90. PWN. Warszawa, $76 \mathrm{pp}$.

Burakowski B.. Mroczkowski M. \& Stefańsia J. 1987. Chrzaszcze Coleoptera. Cucujoidea. cz. 3. Katalog fauny Polski 23 (14): 1-309.

Burakowski B.. Mroczkowski M. \& Stefásska J. 2000. Uzupełnienia tomów 2-21. Katalog fauny Polski 23 (22): 1-252. 
B Yк A. 2001. Interesujące chrząszcze (Coleoptera) odłowione w okolicach Hrubieszowa. Wiadomości Entomologiczne 19 (3/4): 195.

BYк A. \& BYK S. 2004. Chrząszcze saproksylofilne próchnowisk rezerwatu „Dęby w Krukach Pasłęckich". Parki Narodowe i Rezerwaty Przyrody 23 (4): 555-580.

Cieślak R. 2004. Nowe odnotowania Platyscelis melas Fischer. 1824 (Coleoptera: Tenebrionidae) w Polsce. Wiadomości Entomologiczne 23 (1): 56-57.

Dubrovina M. I. 1982. Obzor zhukov-pyltseedov roda Isomira Muls. (Coleoptera, Alleculidae) fauny SSSR. Entomologicheskoe Obozrenie 61 (1): 131-144.

FEJFER F. 1924. Nowe chrząszcze (Coleoptera) dla Ziem polskich. Kosmos 49: 135-143.

FERRER J. 1993. Essai de revision des espèces africaines et européennes appartenant au genre Gonocephalum Solier (Coleoptera. Tenebrionidae). Atti del Museo Civico di Storia Naturale di Trieste 45: 59-150.

FERRER J. 1995. Essai de revision des especes africaines et europeennes appartenant au genre Gonocephalum Solier (Coleoptera. Tenebrionidae). Deuxieme partie. Atti del Museo Civico di Storia Naturale di Trieste 46: 1-75.

FERRER J. \& LundBerg S. 2003. De svenska barksvartbaggarna, släktet Corticeus Piller \& Mitterpacher. 1783 (Coleoptera. Tenebrionidae). Entomologisk Tidskrift 124 (3): 187-192.

Gawroński R. \& Oleksa A. 2007. Nowe stanowiska rzadkich i zagrożonych chrzaszczy (Coleoptera) z północnej Polski. Wiadomości Entomologiczne 26 (1): 5-14.

Gerhardt J. 1910. Verzeichnis der Käfer Schlesiens preussischen und österreichischen Anteils, geordnet nach dem Catalogus coleopterorum Europae vom Jahre 1906. Dritte, neubearbeitete Auflage. J. Springer Verlag. Berlin. 431 pp.

Gosik R. 2007. Description of the pupa of Mvcetochara axillaris (PAYKULI, 1799) (Coleoptera. Tenebrionidae). Baltic Journal of Coleopterology 7 (2): 179-184.

Greń C. 2003. Chrząszcze odłowione w gniazdach ptaków na Śląsku (Coleoptera). Acta Entomologica Silesiana 9/10: 35-40.

Grzywocz J. \& Szoltys H. 1996. Materialy do poznania koleopterofauny Górnego Śląska (Coleoptera). Acta Entomologica Silesiana 4: 14-18.

Gutowski J., Buchiolz L.. Kubisz D., Ossowska M. \& Sućko K. 2006. Chrząszcze saproksyliczne jako wskaźnik odkształceń ekosystemów leśnych borów sosnowych. Leśne Prace Badawcze 4: 101-144.

Gutowski J. M. \& Kubisz D. 1995. Entomofauna drzewostanów pohuraganowych w Puszczy Białowieskiej. Prace Instytutu Badawczego Leśnictwa, Seria A 788: 91-129.

Gutowski J. M., Kubisz D. \& Buchiolz L. 2005. Chrząszcze (Coleoptera) drzewostanów sosnowych w Borach Tucholskich. In: GwoźDzIński K. (ed.). Bory Tucholskie III. Zasoby i ich ochrona. pp. 113-135. Wydawnictwo Uniwersytetu Lódzkiego, Lódź. 276 pp.

Gutowski J. M.. Kubisz D.. Sućko K. \& Zub K. 2010. Sukcesja saproksylicznych chrzaszczy (Coleoptera) na powierzchniach pohuraganowych w drzewostanach sosnowych Puszczy Piskiej. Leśne Prace Badawcze 71 (3): 279-298.

Iwan D. 2004. Tenebrionidae. In: Bogdanowicz W., Chudzicka E., Pilipiuk I. \& Skibińska E. (eds.). Fauna Polski - charakterystyka i wykaz gatunków, tom I. pp. 112-113. Muzeum i Instytut Zoologii PAN. Warszawa, 509 pp.

Iwan D. \& Lobl I. 2008. Opatrini. In: Lobl I. \& Smetana A. (eds.), Catalogue of Palaearctic Coleoptera. Vol. 5. pp. 258-277. Apollo Books. Stenstrup. 670 pp.

JALOSZyŃski P. \& Konwerski S. 2001. Znaczenie parków śródmiejskich w zachowaniu różnorodności gatunkowej chrząszczy (Coleoptera) na przykładzie parku „Cytadela” w Poznaniu. In: InD YKIEWICz P., BARCZAK T. \& KACzorowski G. (eds.), Bioróżnorodność i ekologia populacji zwierzęcych w środowiskach zurbanizowanych. pp. 46-51. NICE. Bydgoszcz. 
Jaloszyński P., Konwerski S. \& Ruta R. 2006. Nowe stanowiska chrząszczy z rodzaju Myrmechixenus Chevrolat (Coleoptera: Tenebrionidae) w Polsce. Wiadomości Entomologiczne 25 (1): 60-61.

Jaskula R. Przewoźny M. \& Melke A. 2009. Chrząszcze (Coleoptera). In: Jaskuta R. \& Tó́czyk G. (eds.). Owady (Insecta) Spalskiego Parku Krajobrazowego. Część I. pp. 27-59. Mazowiecko-Świętokrzyskie Towarzystwo Ornitologiczne. Spala, 112 pp.

KLAsiński J. 2007. Chrząszcze (Coleoptera) rezerwatu Sokole Góry w Jurajskim Parku Krajobrazowym. Biuletyn Częstochowskiego Kola Entomologicznego 6: 3-6.

KLAsiński J. \& Minkina Ł. 2008. Interesujace chrząszcze (Coleoptera) odłowione w okolicach Częstochowy w 2008 roku. Biuletyn Częstochowskiego Kola Entomologicznego 7: 7.

Klejdysz T. \& Kubisz D. 2003. Chrząszcze (Coleoptera) związane z grzybami nadrzewnymi Puszczy Niepołomickiej (Kotlina Sandomierska). Rocznik naukowy Polskiego Towarzystwa Ochrony Przyrody „Salamandra” 7: 145-166.

Konwerski S. 2004. Blaps mortisaga (Linnaeus, 1758). In: Glowací́ski Z. \& Nowacki J. (eds.), Polish Red Data Book of Animals. Invertebrates. pp. 136-137. Instytut Ochrony Przy rody PAN w Krakowie \& Akademia Rolnicza im. A. Cieszkowskiego w Poznaniu. Kraków, 448 pp.

Konwerski S. \& PrzewoźNy M. 2001. Interesujące gatunki chrząszczy (Coleoptera) odłowione w samolówki świetlne w Puszczy Noteckiej. Wiadomości Entomologiczne 20 (1/2): 90.

Kotula B. 1872. Dodatek do fauny chrząszczów Galicyi. Sprawozdania Komisyi Fizyograficznej 6: 69-72.

Kotula B. 1873. Przyczynek do fauny chrzaszczów Galicyi. Sprawozdania Komisyi Fizyograficznej 7: 53-90.

Kotula B. 1874. Przyczynek do fauny chrząszczów galicyjskich. Sprawozdania Komisyi Fizyograficznej 8: 18-26.

KuBisz D. 1995. Chrzaszcze (Coleoptera) z wybranych rodzin jako element monitoringu ekologicznego w Puszczy Białowieskiej. Prace Instytutu Badawczego Leśnictwa. Seria A 797 : 161-176.

Kubisz D. 2000. Mordellochroa milleri EMeRy (Mordellidae). Anaspis bohemica Schilsky (Scraptiidae) i Corticeus bicoloroides (RouBAL) (Tenebrionidae) - nowe dla fauny Polski gatunki chrząszczy (Coleoptera: Tenebrionoidea). Wiadomości Entomologiczne 19 (1): 9-14.

Kubisz D. 2004. Chrzasszcze (Coleoptera) z wybranych rodzin jako element monitoringu ekologicznego w Puszczy Bialowieskiej. Wyniki badań z lat 1993-1999. Leśne Prace Badawcze 4: $37-49$.

Kubisz D.. Hilszczański J. \& Garbalníski P. 2000. Chrzaszcze (Coleoptera) rezerwatów „Czerwińskie Góry” I i II i ich otuliny w Puszczy Kampinoskiej. Parki Narodowe i Rezerwaty Przyrody 19 (4): 83-89.

Kubisz D.. Mazur M. \& Pawlowski J. 1998. Chrzaszcze Miodoborów (Zachodnia Ukraina). II. Aktualny stan poznania. (Insecta: Coleoptera). Studia Ośrodka Dokumentacji Fizjograficznej 25: 217-294.

Kubisz D. \& Szafranec S. 2003. Chrzaszcze (Coleoptera) masywu Babiej Góry. In: Woloszyn B. W. Woeoszyn D. \& Celary W. (eds.). Monografia fauny Babiej Góry, pp. 163-221. Komitet Ochrony Przyrody PAN, Kraków, 489 pp.

Kubisz D. \& Szwalko P. 1994. Chrząszcze (Coleoptera) nowe dla fauny Polski. Wiadomości Entomologiczne 13 (1): 13-19.

Kubisz D.. Szwalko P. \& Wojas T. 1998. Materials to the fauna of Coleoptera of the Western Bieszczady Mts. (Polish Eastern Carpathians). Annals of the Upper Silesian Museum in Bytom. Natural History 15: 5-15. 
Kubisz D. \& Tsinkevich V. A. 2001. Familia (rodzina): Tenebrionidae. In: Gutowski J. M. \& Jaroszewicz B. (eds.), Catalogue of the fauna of Bialowieża Primeval Forest. pp. 182-183. Instytut Badawczy Leśnictwa. Warszawa, 403 pp.

Lobl I. \& Smetana A. (eds.) 2008. Catalogue of Palaearctic Coleoptera. Vol. 5. Apollo Books. Stenstrup, $670 \mathrm{pp}$.

Lültwitz A. 1916. Verzeichnis der im Regierungsbezirk Köslin aufgefundenen Käfer. Stettiner Entomologische Zeitung 76: 205-264.

Makólski J. 1952. Rodzina Carabidae w zbiorze Wojciecha MĄczYńskiego (Coleoptera). Fragmenta Faunistica Musei Zoologici Polonici 6: 207-241.

Marczak D. 2010. Rzadkie gatunki chrząszczy (Insecta: Coleoptera) Kampinoskiego Parku Narodowego. Parki Narodowe i Rezerwaty Przyrody 29 (2): 81-91.

Melke A. \& Grzywocz J. 2003. Carpophilus pilosellus Motschulsky, 1858 (Coleoptera. Nitidulidae) - nowy chrząszcz w polskiej entomofaunie synantropijnej oraz interesujące dane o innych gatunkach tego środowiska. Acta Entomologica Silesiana 11: 102-105.

Novak V. 2005. Icones Insectorum Europae Centralis. Coleoptera: Tenebrionidae. Folia Heyrovskyana. seria B. 2: 1-20.

Paweowski J.. Kubisz D. \& Mazur M. 2002. Coleoptera chrzaszcze. In: Glowaciński Z. (ed.): Red List of Threatened Animals in Poland. pp. 88-110. Instytut Ochrony Przyrody PAN. Kraków, 155 pp.

Pawlowski J., Petryszak B., Kubisz D. \& Szwalko P. 2000. Chrzązzcze (Coleoptera) Bieszczadów Zachodnich. Monografie Bieszczadzkic 8: 9-143.

Przewoźny M. 2007. Chrząszcze (Coleoptera) okolic Jeziora Maltańskiego w Poznaniu. Nowy Pamiętnik Fizjograficzny 5 (1/2): 29-48.

RutA R. 2005. Nowe stanowisko pokatnika zlowieszczka Blaps mortisaga (L.) (Coleoptera: Tenebrionidae) w dolinie Noteci oraz uwagi o występowaniu. biologii. ekologii i zagrożeniu pokątników. Wiadomości Entomologiczne 24 (1): 33-42.

RutA R. 2007. Chrząszcze (Insecta: Coleoptera) kserotermicznych wzgórz Byszewickich w Dolinie Noteci. Nowy Pamiętnik Fizjograficzny 5 (1/2): 49-107.

RyBiński M. 1897. Wykaz chrząszczów nowych dla fauny galicyjskiej. Sprawozdania Komisyi Fizyograficznej 32: 46-62.

Rybiśski M. 1902. Coleopterorum species novae minusqve cognitae in Galicia inventae. Rozprawy Wydzialu Matematyczno-Przy rodniczego Akademii Umiejętności 42, seria B: 1-8.

Rybiński M. 1903a. Chrząszcze nowe dla fauny galicyjskiej. Wykaz II. Sprawozdania Komisyi Fizyograficznej 37: 15-30.

RyBiński M. 1903b. Wykaz chrząszczów zebranych na Podolu galicyjskiem przy szlaku kolejowym Złoczów - Podwoloczyska w latach 1884-1890. Sprawozdania Komisyi Fizyograficznej 37: 57-175.

SeIDlitz G. 1896. Tenebrionidae (pars III). In: Naturgeschichte der Insekten Deutschlands. Berlin. 5. 1, 4: 609-800.

Sienkiewicz P. \& Konwerski S. 2005. Rare and endangered beetles (Coleoptera) from Krajkowo Nature Reserve in the middle course of the Warta river in Western Poland. In: SkLoDowsKi J., Huruk S., Barśsevskis A. \& Tarasiuk S. (eds.), Protection of Coleoptera in the Baltic Sea Region. pp. 57-63. Warsaw Agricultural University Press, Warsaw. 239 pp.

SMrEczyński S. 1932. Zbiór ryjkowców ś.p. Wojciecha MĄczÝ́skiego. Sprawozdania Komisji Fizjograficznej 65: 1-24.

Stebnicka Z. 1991. Czarnuchowate - Tenebrionidae. Boridae. Klucze do oznaczania owadów Polski. cz. XIX Chrząszcze - Coleoptera, z. 91. Polskie Towarzystwo Entomologiczne. Wroclaw, $93 \mathrm{pp}$. 
SToвIEcki S. 1883. Do fauny Babiej góry. Sprawozdanie z wycieczek entomologicznych na Babią Górę w latach 1879 i 1880. Sprawozdania Komisyi Fizyograficznej 17 (II): 1-84.

Stobecki S. 1939. Chrzaszcze (Coleoptera) ś.p. Wojciecha Mączý́skiego w zbiorach entomologicznych Śląskiego Muzeum Przyrodniczego w Katowicach. Kózki (Cerambycidae). Sprawozdania Komisji Fizjograficznej 72: 263-268.

Szujecki A. 1958. Zbiór chrzaszczy Wojciecha Mącz yńskiego. Steninae (Coleoptera. Staphylinidae). Polskie Pismo Entomologiczne. 27: 115-119.

Szymczakowski W. 1959. Zbiór chrząszczy Wojciecha MĄczý́skiego. Catopidae (Coleoptera). Polskie Pismo Entomologiczne. 29: 195-199.

Szymczakowski W. 1960. Materialy do poznania kserotermofilnej fauny chrząszczy Wyżyny Małopolskiej. Polskie Pismo Entomologiczne 30 (14): 173-242.

Szymczakowski W. 1965. Materialy do poznania chrzaszczy (Coleoptera) siedlisk kserotermicznych Polski. Polskie Pismo Entomologiczne 35 (5): 225-257.

Szymczakowski W. 1973. Dalsze materiały do znajomości chrząszczy (Coleoptera) biotopów kserotermicznych Polski. Acta Zoologica Cracoviensia 18 (9): 183-216.

Tenenbaum S. 1913. Chrząszcze (Coleoptera) zebrane w Ordynacyi Zamojskiej w gub. Lubelskiej. Pamiętnik Fizyograficzny 21: 1-72.

Tenenbaum S. 1918. Dodatek do spisu chrzaszczy z Ordynacyi Zamojskiej. Pamiętnik Fizyograficzny $25: 1-35$.

Tenenbaum S. 1923. Przybytki do fauny chrząszczów Polski od roku 1913. Rozprawy i Wiadomości z Muzeum im. Dzieduszyckich 7/8: 136-186.

TenenBaum S. 1931. Nowe dla Polski gatunki i odmiany chrząszczy, oraz nowe stanowiska gatunków dawniej podawanych. V. Fragmenta Faunistica Musei Zoologici Polonici 1: 329-359.

Trella T. 1923. Wykaz chrząszczów okolic Przemyśla. Heteromera. Polskie Pismo Entomologiczne 2: 12-19.

TSHeRniey L. S. 2005. Zhuki-tshernotelki (Coleoptera, Tenebrionidae). Fauna Ukrainy, t.19, vyp. 10. Naukova Dumka, Kiev, 432 pp.

TYKarski P. 2006. Beetles associated with scolytids (Coleoptera. Scolytidae) and the elevational gradient: Diversity and dynamics of the community in the Tatra National Park. Poland. Forest Ecology and Management 225: 146-159.

TYKARSKi P. 2010. Scope and applicability of information stored in natural history collections a case study based on the largest museum collections of Tenebrionidae (Coleoptera) in Poland. Fragmenta Faunistica 53(1): 97-115.

Uechtritz [M.] 1848. [In Schlesien sehr seltene, oder gar nicht gefundene Käfer]. Uebersicht der Arbeiten und Veränderungen der Schlesischen Gesellschaft für Vaterlandische Kultur, Breslau 1847: 104-105. 


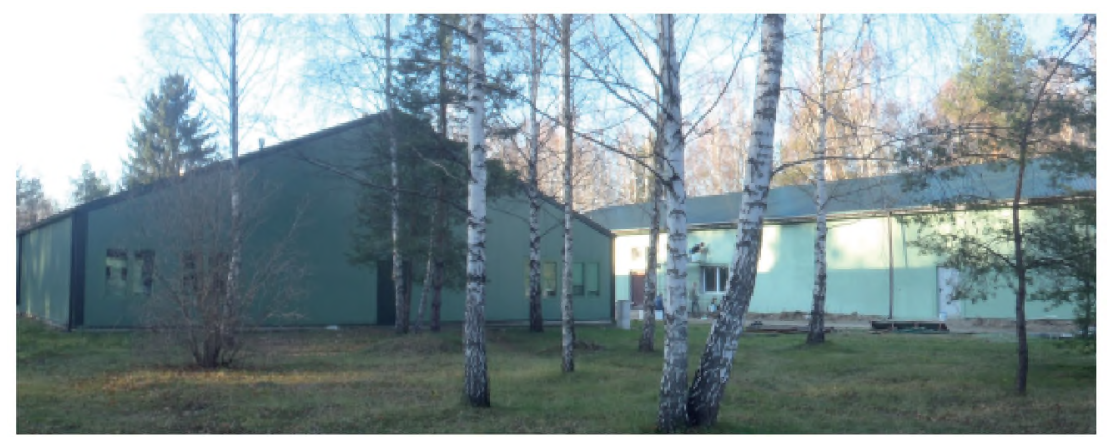

Fig. 1. Zoological collections in Lomna. Museum and Institute of Zoology PAS, Warsaw (photo D. IwAN).

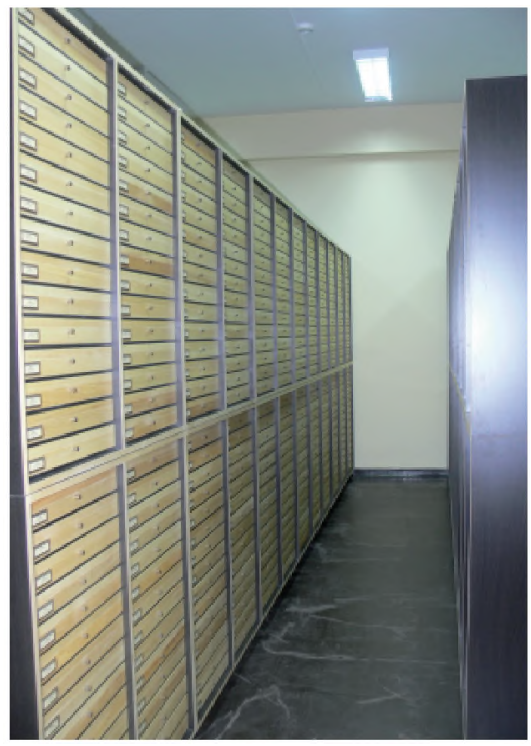

Fig. 2. Insects collections. Upper Silesian Museum, Bytom (photo R. DoBosz).

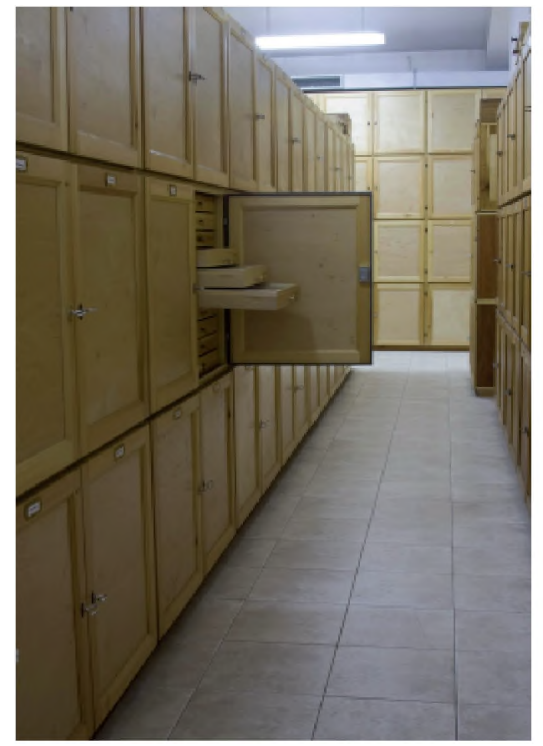

Fig. 3. Insects collections. Institute of Systematics and Evolution of Animals PAS, Cracow (photo E. Przybyeowicz).

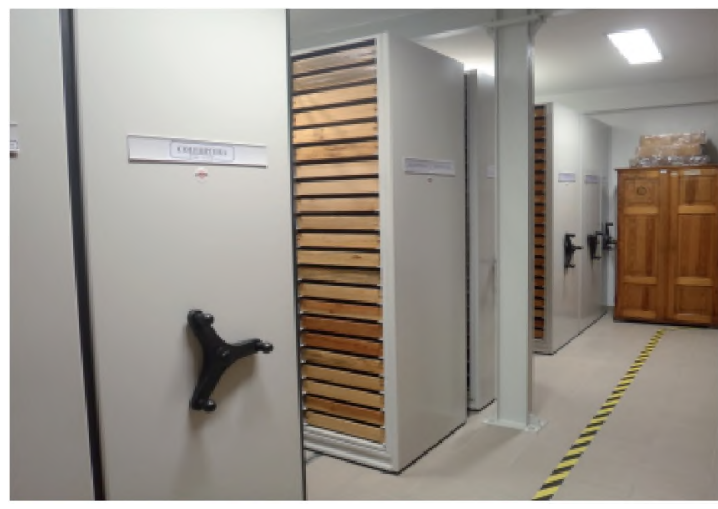

Fig. 4. Insects collections, Łomna. Museum and Institute of Zoology PAS, Warsaw (photo D. IwAN). 


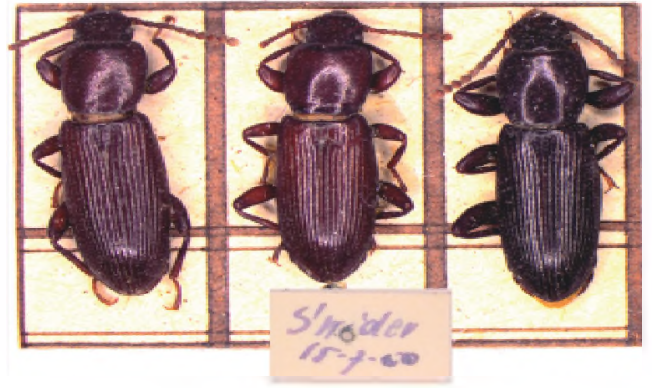

Fig. 5. Centorus elongatus (Herbst), USMB collection (photo D. KuBISZ).
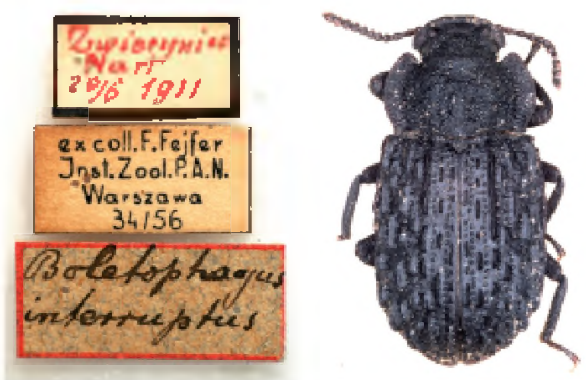

Fig. 6. Bolitophagus interruptus ILL. MLZ collection (photo D. SCHIMrosczyk).

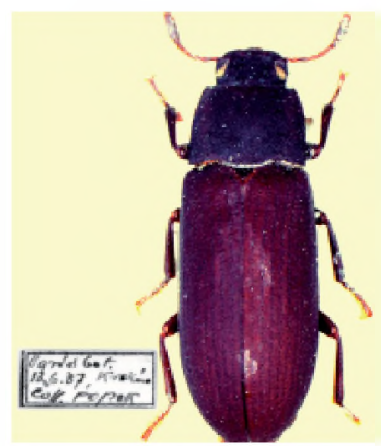

Fig. 7. Cryphaeus cormutus (FISH.), ISEA collection (photo D. KuBIsz).

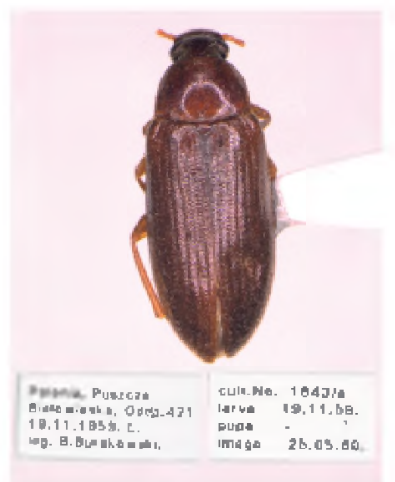

Fig. 10. Hymenophorus doublieri MuLs., MIZ collection (photo D. KuBisz).

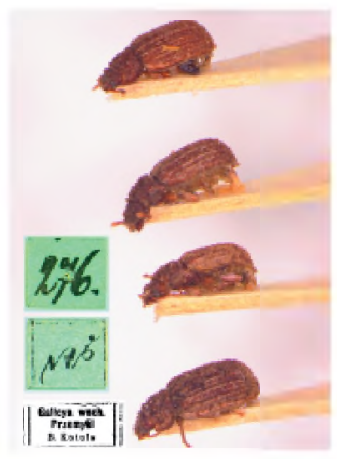

Fig. 8. Eledonoprius armatus (PANZ.), ISEA collection (photo D. Kubisz).
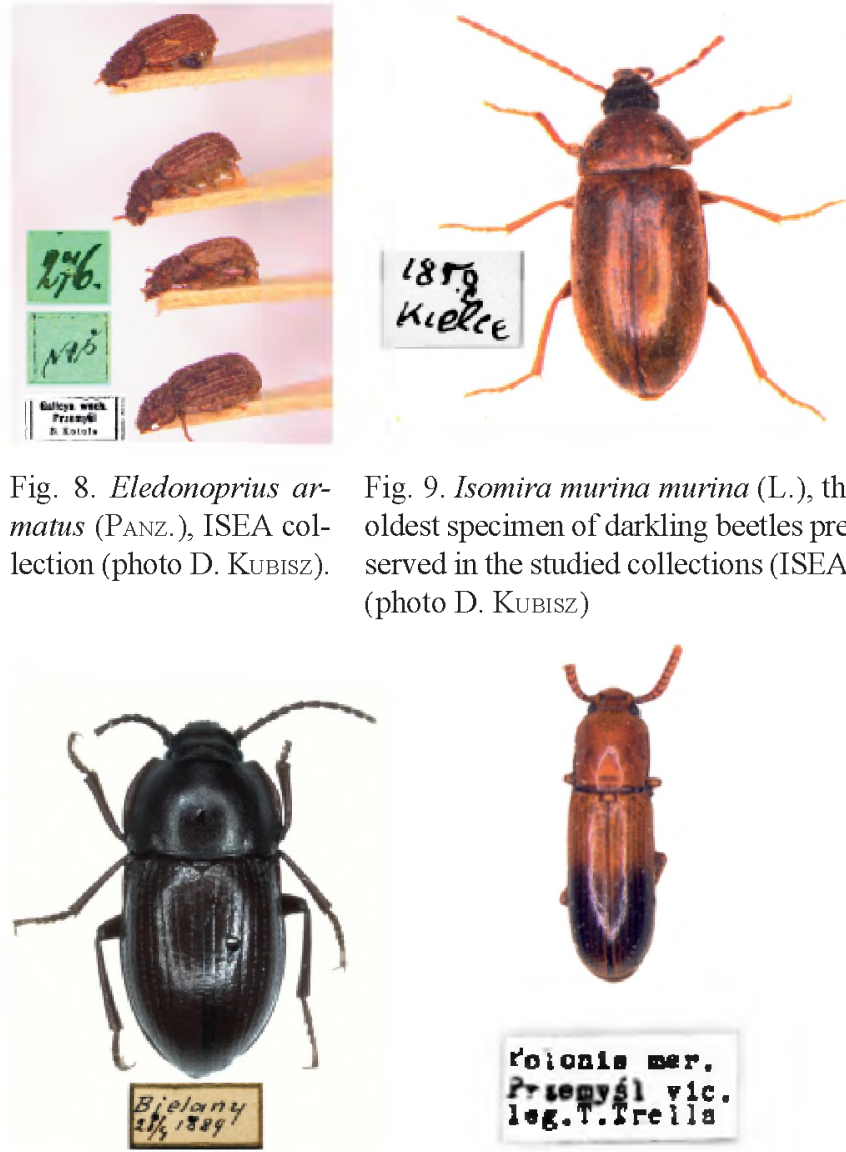

Fig. 9. Isomira murina murina (L.), the oldest specimen of darkling beetles preserved in the studied collections (ISEA) (photo D. Kubisz)
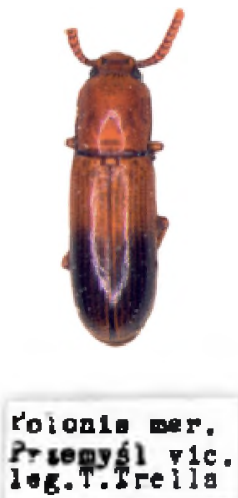

Fig. 11. Nalassus convexus (COM.), USMB collection (photo D. SCHIMROSCZYK).
Fig. 12. Corticeus bicoloroides (RoubaL), ISEA collection (photo D. KuBISz). 


\section{INDEX OF NAMES}

aeneus, Stenomax 2, 32

agricola, Eledona 16

ALLECULINAE 45

Alleculini 45

Alphitobiini 23

armatus, Eledonoprius 17, 83, 84, 91

ater, Prionychus 57

atripes, Lagria 8

axillaris, Mycetocharis 52

badius, Odocnemis 85

Belopini 12

betulae, Omophlus 62

bicolor, Corticeus 74

bicolor, Scaphidema metallicum 83

bicoloroides. Corticeus 75, 91

bifasciatus, Alphitophagus 64, 83

Blaps 34

Blaptini 33

boleti, Diaperis 65

Bolitophagini 14

cadaverina, Phaleria 84

castaneum, Tribolium 24

ceramboides, Pseudocistela ceramboides 59

ceramboides, Upis 85

chrysomeloides, Pentaphyllus 84

ciscaucasica, Isomira murina 52

confusum, Tribolium 25

convexus, Nalassus 1, 12, 29, 85, 91

cornutus, Cryphaeus 1, 12, 14, 85, 91

cornutus, Gnatocerus 67

Corticeus 81

Crypticini 70

Cteniopodini 61

culinaris, Uloma 26

cylindricus, Menephilus 84

dejeanii, Platydema 69,77, 83

dentipes, Enoplopus 85

depressus, Palorus 12

dermestoides, Nalassus 2, 29

destructor, Tribolium 25
DIAPERINAE 64

Diaperini 64

diaperinus, Alphitobius 23

doublieri, Hymenophorus 48, 83, 84, 91

ecalcaratus, Centorus elongatus 12

elongatus, Centoms elongatus 1, 12, 85, 91

fasciatus, Corticeus 76

femoralis, Pedinus 36

flavipes, Mvcetocharis 53

flavus, Cteniopus 61

fraxini, Corticeus 76

gibbus, Phylan 36

haemorrhoidalis, Neomida 67, 69, 84

halophila. Blaps 33, 83, 84

hellenica, Gonodera luperus 47

Helopini 29

helopioides, Pedinus 1, 73, 84

hirta, Lagria 8

humeralis, Mycetocharis 55

Hypophlaeini 74

icteropa, Isomira 1, 50, 52, 84

interruptus, Bolitophagus 14, 83, 84, 91

Laenini 7

laevigatus, Alphitobius 23, 83

laevioctostriatus, Nalassus 31

LAGRIINAE 7

Lagriini 8

lanipes, Stenomax 2, 33

lepturoides, Omophlus 85

lethifera, Blaps lethifera 34, 84

linearis, Corticeus 77, 79, 84

linearis, Mvcetocharis 56

lividipes, Omophlus 84

longulus, Corticeus 78

luperus, Gonodera luperus 47

madens, Tribolium 26

maura, Mycetocharis 56

melanarius, Prionychus 58

Melanimini 42

melas, Oodescelis 42, 83, 84

metallicum, Scaphidema metallicum 81 
molitor, Tenebrio 19 morio, Allecula 45, 47

mortisaga, Blaps 34.84

mucronata, Blaps 35, 84

murina, Isomira murina 1, 2, 49, 83, 84, 91

Myrmechixenini 73

nigrita. Podonta 64, 84

nomas, Tentyria 84

obscura, Mycetocharis 84, 85

obscurus, Tenebrio 21

obtusa, Prosodes 84

Omophlus 63

opacus, Tenebrio $22,83,84$

Opatrini 37

oryzae, Latheticus 24,84

Palorini 12

Pedinini 36

picinus, Myrmechixenus 85

picipes, Helops 2

picipes, Neatus 18

picipes, Omophlus 85

pini, Corticeus 78

Platyscelidini 42

polita. Oodescelis 42,84

pterotapha, Blaps lethifera 34

pubescens, Omophlus 62

pusillum, Gonocephalum granulatum 37, 84

pygmaea, Mycetocharis 56

quisquilius, Crypticus 1, 70, 84

ratzeburgii, Palorus 13,84

reitteri, Laena 7, 83, 84

reticulatus, Bolitophagus 14 rhenana, Allecula 46, 83

riparium, Opatrum 38

roubali, Mycetocharis 84

rufa, Uloma 27

rufipes. Hymenalia 48

sabulosa, Asida 84

sabulosum, Opatrum sabulosum 39

Scaphidemini 81

semiflava, Isomira 1, 2, 52, 84

serrata, Pseudocistela ceramboides 60

spinimanus, Gnaptor 84

subaereus, Melanimon tibialis 45

subalpina, Blaps lethifera 34

subdepressus, Palorus 13, 84

suberis, Corticeus 77, 79, 84

subterraneus, Myrmechixenus 73

sulphureus, Cteniopus 61

sulphuripes, Cteniopus 62, 84

suturalis, Corticeus 79

Tenebrio 18

TENEBRIONINAE 12

Tenebrionini 18

testaceus, Pentaphyllus 68

thoracicus, Bius 84

tibialis, Melanimon tibialis 42

Toxicini 14

Triboliini 24

Tribolium 26

Ulomini 26

unicolor, Corticeus 79

vaporariorum, Myrmechixenus 84

velikensis, Accanthopus 85

versipellis, Corticeus 85

violaceum, Platydema 70 


\section{STRESZCZENIE}

\section{[Występowanie Tenebrionidae (Coleoptera) w Polsce na podstawie największych krajowych kolekcji muzealnych]}

W niniejszej pracy przedstawiono wyniki badań nad chrzaszczami z rodziny czarnuchowatych przechowywanych w największych polskich kolekcjach zoologicznych, w następujących instytucjach: Instytut Systematyki i Ewolucji Zwierzat PAN w Krakowie (ISEA), Muzeum i Instytut Zoologii PAN w Warszawie (MIZ) oraz Muzeum Górnośląskie w Bytomiu (USMB).

Przebadany material zawiera ponad 10,500 okazów należących do 79 gatunków zebranych z terenu Polski.

Najstarszym (datowanym) okazem, zachowanym w opracowanych zbiorach, jest egzemplarz Isomira murina (L.), zebrany przez Antoniego Wagę w Kielcach, w 1859 roku. Można więc przyjać, że dane z opracowanych zbiorów obejmuja okres ostatnich 150 lat.

Studia faunistyczne, przeprowadzane w oparciu o powyższy material, dostarczyly następujących danych na temat czarnuchowatych wykazywanych z Polski:

- potwierdzono występowanie 14 gatunków rzadko odlawianych, takich jak: Blaps halophila Fisch., Oodescelis melas (Fisch.), Bolitophagus interruptus Ill., Eledonoprius armatus (Panz.), Platydema dejeanii (Cast. \& Br.), Alphitophagus bifasciatus (Say), Alphitobius laevigatus (Fabr.), Tenebrio opacus (Duft.), Laena reitteri Weise, Allecula rhenana Bach, Hymenophorus doublieri Muls., Cteniopus sulphuripes (Germ.), Gonocephalum granulatum pusillum (Fabr.) i Latheticus oryzae Water.

- odnotowano obecność okazów należących do 14 gatunków umieszczonych w „Polskiej Czerwonej Księdze Zwierząt” (Konwerski 2004) oraz „Czerwonej liście zwierząt ginących i zagrożonych w Polsce" (Pawlowski i in. 2002): Blaps halophila Fisch., B. lethifera Marsh., B. mortisaga (L.), B. mucronata Latr., Oodescelis melas (Fisch.), Hymenophorus doublieri Muls., Cteniopus sulphuripes (Germ.), Podontanigrita (Fabr.), Bolitophagus interruptus Ill., Eledonoprius armatus (Panz.), Neomida haemorrhoidalis (Fabr.), Corticeus suberis (Lucas), Tenebrio opacus Duft. i Laena reitteri Weise; nie odnotowano natomiast innych gatunków znajdujących się w wyżej wymienionych publikacjach: Gnaptor spinimanus (Pal.), Bius thoracicus (Fabr.), Mycetocharis obscura (Zeitt.), M roubali Mařan i Menephilus cylindricus (Herbst);

- pospolicie występujący gatunek Isomira murina (L.), który jest starszym synonimem I. semiflava (Küst.), powinien być wylączony z "Czerwonej listy zwierząt ginących i zagrożonych w Polsce" (Pawłowski i in. 2002);

- wykazano blędne oznaczenia okazów należących do 4 gatunków rzadkich lub w Polsce niewystepujacych: Crypticus quisquilius (L.), a nie Pedinus helopioides Ahr., jeden okaz przechowywany w zbiorach MIZ, zebrany przez F. Fejfera (Roztocze, Florianka), cytowany przez Tenebauma $(1918,1923)$ i KFP (Catalogus faune Poloniae, Burakowski et al. 1987); Isomira murina (L.), a nie Isomira icteropa (Küst.), jeden okaz przechowywany w zbiorach ISEA, zebrany w okolicach Ojcowa i cytowany przez 
Szymczakowskiego (1973) oraz KFP; oba gatunki nie powinny być zaliczane do fauny Polski; Oodescelis melas (Fisch.), a nie Oodescelis polita (Sturm), 2 okazy (Radomyśl) wykazane z Polski przez Szymczakowskiego (1973) oraz KFP; Palorus subdepressus Woll., a nie Palorus ratzeburgii (Wissm.), jeden okaz zdeponowany w zbiorach MIZ, zebrany przez B. Burakowskiego (Lomianki) i wykazywany w KFP; Corticeus linearis (Fabr.), a nie Corticeus suberis (Lucas), jeden okaz znajduje się w kolekcji MIZ, zebrany przez F. Fejfera (Roztocze, Florianka) i zacytowany w 1924 roku oraz w KFP;

- w opracowanych kolekcjach nie wykazano okazów gatunków, których występowanie w Polsce wymaga potwierdzenia: Gnaptor spinimanus (Pal.), Myrmechixenus vaporariorum Guér., Bius thoracicus (Fabr.), Menephilus cylindricus (Herbst), Mycetocharis roubali Mařan, Omophlus lividipes Muls., Tentyria nomas (Pal.), Asida sabulosa (Fues.), Prosodes obtusa (Fabr.), Phaleria cadaverina (Fabr.), Pentaphyllus chrysomeloides (Rossi), Myrmechixenus picinus (Aubé), Corticeus versipellis (Baudi), Upis ceramboides (L.), Accanthopus velikensis Pill. \& Mitt. [=Enoplopus dentipes (Rossi)], Odocnemis badius (Redt.), Mycetocharis obscura (Zeitt.), Omophlus lepturoides (Fabr.) i O. picipes (Fabr);

- dla 44 gatunków przedstawiono nowe dane o występowaniu w krainach nie wykazywanych w KFP (Burakowski et al. 1987);

- odnotowano obecność w zbiorach okazów złowionych w Polsce, należących do gatunków, których granice zasięgów występowania są znacznie oddalone od terytorium naszego kraju: Centorus elongatus (Herbst), Cryphaeus cornutus (Fish.) i Nalassus convexus (Com.); prawdopodobnie okazy te zostaly zawleczone, stąd nie należy wlączać ich w obecnej chwili do fauny Polski.

W równoleglym artykule (zeszyt 1, tom 53, Fragmenta Faunistica) zostaly zaprezentowane (Tykarski 2010) dodatkowe analizy wykonane w oparciu o dane pochodzące z niniejszej pracy. 